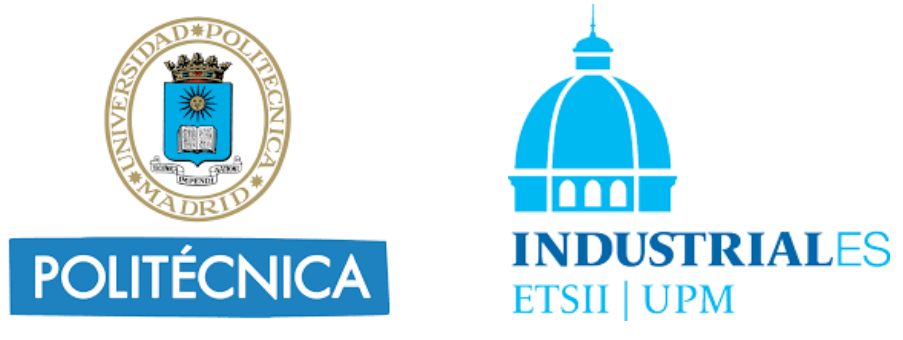

DEPARTAMENTO INGENIERÍA MECÁNICA

ESCUELA TÉCNICA SUPERIOR DE INGENIEROS INDUSTRIALES

\title{
EVALUACIÓN DE TERAPIAS ACÚSTICAS COMO TRATAMIENTO DE TINNITUS CRÓNICO Y REFRACTARIO
}

\author{
Autor: \\ Francisco José Tavira Sánchez \\ Ingeniero de Telecomunicación \\ Máster en Ingeniería Acústica \\ Máster en Administración de Empresas \\ Profesor de Violín
}

\author{
Directores: \\ Dr. Manuel Recuero López \\ Doctor en Ciencias Físicas \\ Dr. David Isaac Ibarra Zárate \\ Doctor Universidad Politécnica de Madrid
}





\section{TRIBUNAL TESIS DOCTORAL}

\section{EVALUACIÓN DE TERAPIAS ACÚSTICAS COMO TRATAMIENTO DE TINNITUS CRÓNICO Y REFRACTARIO}

AUTOR: Francisco José Tavira Sánchez

DIRECTORES: Manuel Recuero López y David Isaac Ibarra Zárate

Tribunal nombrado por el Excmo. y Magfco. Sr. Rector de la Universidad Politécnica de Madrid, el día de de 2019.

\begin{tabular}{|c|c|}
\hline Presidente & $\mathrm{D} . / \mathrm{D}^{\mathrm{a}}$. \\
\hline Secretario & $\mathrm{D} . / \mathrm{D}^{\mathrm{a}}$. \\
\hline Vocal & 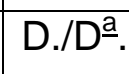 \\
\hline Vocal & $\mathrm{D} . / \mathrm{D}^{\mathrm{a}}$. \\
\hline Vocal & $\mathrm{D} . / \mathrm{D}^{\mathrm{a}}$. \\
\hline Suplente & $\mathrm{D} . / \mathrm{D}^{\mathrm{a}}$. \\
\hline Suplente & $\mathrm{D} . / \mathrm{D}^{\mathrm{a}}$. \\
\hline
\end{tabular}

Realizado el acto de defensa el día de de 2019 en la Escuela

Técnica Superior de Ingenieros Industriales de la Universidad Politécnica de Madrid.

Calificación obtenida:

EL PRESIDENTE

SECRETARIO

VOCAL

VOCAL

VOCAL 



\section{TESIS DOCTORAL}

Título Evaluación de terapias acústicas como tratamiento de Tinnitus crónico y refractario

Autor

Francisco José

Tavira Sánchez

Directores

Dr. Manuel

Recuero López

Dr. David Isaac Ibarra Zárate

Tutor

Ídem

Informadores expertos internacionales
Dra. Luz María Alonso Valerdi
Ídem

Máster en Ingeniería Acústica

Universidad Politécnica de Madrid

Doctor en Ciencias Físicas

Profesor Emérito

Catedrático de Universidad

Grupo de Investigación en

Instrumentación y Acústica Aplicada

Universidad Politécnica de Madrid

Doctor en Ingeniería Acústica

Profesor Investigador

Tecnológico de Monterrey, Campus

Puebla

Grupo de Investigación en

Neuroingeniería y Neuroacústica

Doctora en Computación y Electrónica

Profesor Investigador

Tecnológico de Monterrey, Campus

Monterrey

Grupo de Investigación en

Neuroingeniería y Neuroacústica 

To my daughters:

Olivia Elodie and Emilia Athenea 


\section{ACKNOWLEDGEMENTS}

First of all, I would like to thank Dr. Manuel Recuero and Dr. David Ibarra for their guidance and support. They have helped me not only with their vision, knowledge and experience but also with their patience, respect and affection.

Second of all, my wife, daughters and parents who have been the strength and light in this path and without them nothing would have been possible. And our cat, to share the long nights with me.Thanks for understanding every step of this doctorate (even the worst ones).

Apart of that, I need to dedicate some special words to

- the team who I started this "adventure" with and under the lead of Dr. Manuel Recuero: Dra. Adela Crespo, Dr. Gerardo Galvez, Adrián Begoña and Jose Luis Sánchez.

- all the places and people partially or fully involved during all these years:

- Escuela de Ingeniería y Ciencias of Tecnológico de Monterrey (TEC): Dr. Ricardo Ramirez for believing in this project, and Dra. Luz María Alonso for her significant collaboration.

- Instituto Nacional de Rehabilitación (INR): Laura Hernádez and the patients engaged.

- Grupo de Investigación en Instrumentación y Acústica Aplicada (I2A2) of Universidad Politécnica de Madrid, Spain (UPM): Guillermo De Arcas and Ignacio Pavón.

- Laboratory of Cognitive and Computational Neuroscience (CTB): Fernando Maestú and Francisco del Pozo (Requiescat In Pace).

- CSIC - Centro de Acústica Aplicada y Evaluación no Destructiva (CAEND).

- Laboratory for Manufacturing and Productivity, Massachusetts Institute of Technology (MIT): Brian Anthony.

- Asociación Parkinson Madrid and their team with whom I began the research.

- Javier Maqueda, Ana de Andrade and Francisco Estevez for listening, embracing and inspiring me.

- Janet, Mauricio, Germán, Jorge, Ruben, Nacho, Jay, Juan, Annet, Stephan, Elke, Santi, Nayeli, Fredes, Javi, Lidón, Julien, Tomeu, Abhi, Alba, Mariana, Alfonso, Álvaro, Vincent, Thierry, Alex, David, Wouter, Heiko, Giuseppe, Aleix, Inga, Paul and everybody who was participating, supporting me or trusting that one day I would achieve it.

- All my remaining friends for staying there still during my "anti-social" life. 


\section{GENERAL INDEX}

ACKNOWLEDGEMENTS III

GENERAL INDEX $\quad$ V

$\begin{array}{lll}\text { ABSTRACT } & \text { XI }\end{array}$

ACRONYMS AND ABBREVIATIONS XIII

1. INTRODUCTION 1

1.1 TINNITUS: MEDICAL PROBLEM TO SOLVE 4

$\begin{array}{lll}\text { 1.1.1 EPIDEMIOLOGY } & 6\end{array}$

$\begin{array}{lll}1.1 .2 & \text { ECONOMIC IMPACT } & 6\end{array}$

$\begin{array}{lll}1.2 & \text { STATE OF THE ART } & 7\end{array}$

$\begin{array}{lll}\text { 1.2.1 } & \text { GAPS AND UNKNOWING } & 10\end{array}$

1.3 HYPOTHESIS, GENERAL AND SPECIFIC OBJECTIVES 11

$\begin{array}{lll}\text { 1.3.1 HYPOTHESIS } & 11\end{array}$

$\begin{array}{lll}\text { 1.3.2 OBJECTIVES } & 11\end{array}$

2. MATERIALS AND METHODS 13

$\begin{array}{llr}2.1 & \text { INTRODUCTION } & 15\end{array}$

$\begin{array}{lll}2.2 & \text { SAMPLE } & 15\end{array}$

$\begin{array}{ll}2.3 \text { GENERATION OF THE ACOUSTIC THERAPIES } & 16\end{array}$

$\begin{array}{lr}2.4 \text { FACILITIES } & 18\end{array}$

$\begin{array}{lll}2.4 .1 & \text { LABORATORY } & 18\end{array}$

$\begin{array}{lll}2.4 .2 & \text { EQUIPMENT AND SOFTWARE } & 19\end{array}$

2.4.3 DEVICES FOR ACOUSTIC THERAPIES 20

2.5 ELECTROENCEPHALOGRAPHIC EVALUATION 21

2.5.1 SPONTANEOUS ACTIVITY AT RESTING STATE 21 
2.5.2 EVOKED ACTIVITY: EVENT-RELATED POTENTIALS (ERPs) 22

$\begin{array}{lll}2.5 .3 & \text { INDUCED ACTIVITY } & 23\end{array}$

2.5.4 OTHER EEG MEASURES 23

2.5.5 ANALYSIS STRATEGY 23

$\begin{array}{lll}2.5 .6 & \text { ANALYSIS TOOLS } & 25\end{array}$

$\begin{array}{llr}2.6 & \text { PROCEDURE } & 25\end{array}$

$\begin{array}{lll}\text { 2.6.1 STUDY DESIGN } & 25\end{array}$

$\begin{array}{lll}\text { 2.6.2 POST-STUDY TREATMENT } & 30\end{array}$

2.6.3 LIMITATIONS AND TROUBLESHOOTING 30

$\begin{array}{lll}2.7 & \text { ETHICS } & 31\end{array}$

$\begin{array}{ll}\text { 3. RESULTS } & 33\end{array}$

$\begin{array}{lll}3.1 & \text { INTRODUCTION } & 35\end{array}$

$\begin{array}{lll}3.2 & \text { SUBJECTIVE ANALYSIS } & 35\end{array}$

$\begin{array}{lll}3.2 .1 & \text { TINNITUS PERCEPTION EVOLUTION } & 37\end{array}$

3.2.2 ANXIETY EVOLUTION 39

$\begin{array}{lll}\text { 3.2.3 DEPRESSION EVOLUTION } & 40\end{array}$

$\begin{array}{lll}3.3 & \text { OBJECTIVE ANALYSIS } & 41\end{array}$

$\begin{array}{lll}\text { 3.3.1 PULSE RATE } & 41\end{array}$

3.3.2 EEG DATA PROCESING AND MEASUREMENTS 41

4. CONCLUSIONS

$\begin{array}{lll}4.1 & \text { INTRODUCTION } & 53\end{array}$

$\begin{array}{lll}4.2 & \text { SUBJECTIVE ANALYSIS CONCLUSIONS } & 54\end{array}$

4.2.1 RELATIVELY EFFECTIVE ACOUSTIC THERAPY: TRT 55

4.2.2 MODERATE ACOUSTIC THERAPY: BINAURAL $396 \mathrm{HZ}$ AND EAE 56

4.2.3 NOT RECOMMENDED TREATMENT: RS AND BINAURAL $F_{0} \quad 56$

$\begin{array}{lll}4.3 & \text { OBJECTIVE ANALYSIS CONCLUSIONS } & 57\end{array}$ 
4.3.1 RELATIVELY EFFECTIVE ACOUSTIC THERAPY: BINAURAL F $F_{0}$ AND 396HZ 58

4.3.2 MODERATE ACOUSTIC THERAPY: TRT 58

4.3.3 NOT RECOMMENDED TREATMENT: RS AND EAE 58

4.4 SUBJECTIVE AND OBJECTIVE ANALYSIS CONCLUSIONS 59

$\begin{array}{ll}\text { CONTRIBUTIONS } & 61\end{array}$

$\begin{array}{ll}\text { QUALITY INDICATORS } & 65\end{array}$

IN-EAR MEDICAL DEVICES FOR ACOUSTIC THERAPIES IN TINNITUS $\begin{array}{ll}\text { TREATMENTS, STATE OF THE ART - JCR (Q2) } & 67\end{array}$

ELECTROENCEPHALOGRAPHIC EVALUATION OF ACOUSTIC THERAPIES FOR THE TREATMENT OF CHRONIC AND REFRACTORY TINNITUS - JCR (Q2)

$\begin{array}{ll}\text { FUTURE RESEARCH TOPICS } & 71\end{array}$

$\begin{array}{ll}\text { BIBLIOGRAPHIC REFERENCES } & 75\end{array}$

$\begin{array}{ll}\text { INDEXES } & 97\end{array}$

$\begin{array}{ll}\text { FIGURES } & 99\end{array}$

$\begin{array}{ll}\text { TABLES } & 101\end{array}$

$\begin{array}{ll}\text { ANNEXES } & 103\end{array}$

$\begin{array}{ll}\text { ANNEX A: MUSIC AS A THERAPY IN EARLY HISTORY } & 105\end{array}$

$\begin{array}{ll}\text { ANNEX B: TINNITUS MODELS } & 106\end{array}$

ANNEX C: TINNITUS DIAGNOSTIC AND THERAPEUTIC MANAGEMENT OF TINNITUS

$\begin{array}{ll}\text { ANNEX D: TINNITUS DEMOGRAPHICS } & 108\end{array}$

ANNEX E: BINAURAL BEATS SUMMARY INFORMATION 110

ANNEX F: SAMPLE OF ACOUSTIC THERAPIES ANALYSIS 111

ANNEX G: AUDIO PLAYER TECHNICAL DETAILS AND DISTORTION 112

$\begin{array}{ll}\text { ANNEX H: UNCERTAINTY CALCULATION } & 113\end{array}$

ANNEX I: BRAIN OSCILLATIONS QUANTIFIED IN TINNITUS 114

$\begin{array}{ll}\text { ANNEX J: EEG ANALYSIS } & 115\end{array}$ 
ANNEX K: SIGNAL-PROCESSING TOOLS

$\begin{array}{ll}\text { ANNEX L: EVENT-RELATED (DE) SYNCHRONIZATION } & 120\end{array}$

ANNEX M: COMMON TINNITUS QUESTIONNAIRES 122

$\begin{array}{ll}\text { ANNEX N: ONLINE QUESTIONNAIRES } & 124\end{array}$

$\begin{array}{ll}\text { ANNEX O: PROTOCOL APPROVAL DOCUMENTATION } & 125\end{array}$

ANNEX P: PATIENT SCORE EVOLUTION FROM QUESTIONNAIRES 126

ANNEX Q: TINNITUS PERCEPTION EVOLUTION CONCLUSIONS ACCORDING TO THE DIFFERENT INITIAL CIA.EVO

ANNEX R: ANXIETY EVOLUTION ADDITIONAL DATA 129

$\begin{array}{ll}\text { ANNEX S: DEPRESSION EVOLUTION ADDITIONAL DATA } & 130\end{array}$

ANNEX T: COMMON STATISTICAL FEATURES IN THE TIME DOMAIN'

ANNEX U: COMMON STATISTICAL FEATURES IN THE FREQUENCY DOMAIN $\quad 134$

ANNEX V: WICA ALGORITHM FOR ARTIFACT SUPPRESSION IN EEG 135

ANNEX W: SESSION 4 - SESSION 1 DIFFERENCE OF TIME AND TREQUENCY DOMAIN PARAMETERS FROM EACH ACOUSTIC THERAPY FOR EO

ANNEX X: SESSION 4 - SESSION 1 DIFFERENCE OF TIME AND TREQUENCY DOMAIN PARAMETERS FROM EACH ACOUSTIC THERAPY FOR EC: SELECTION OF PARAMETERS FOR FRONTAL AND TEMPORAL LOBE

ANNEX Y: SESSION 4 - SESSION 1 DIFFERENCE OF TIME AND TREQUENCY DOMAIN PARAMETERS FROM EACH ACOUSTIC THERAPY FOR EC

ANNEX Z: SESSION 4 - SESSION 1 DIFFERENCE OF TIME AND TREQUENCY DOMAIN PARAMETERS FROM EACH ACOUSTIC THERAPY FOR THERAPY: SELECTION FOR FRONTAL AND TEMPORAL LOBE CHANNELS

ANNEX AA: SESSION 4 - SESSION 1 DIFFERENCE OF TIME AND TREQUENCY DOMAIN PARAMETERS FROM EACH ACOUSTIC THERAPY FOR THERAPY

ANNEX AB: GRAND AVERAGE ERP OF 16 CHANNELS FROM ALL PATIENTS' SESSION 1 AND SESSION 4 OF EACH ACOUSTIC THERAPY

ANNEX AC: EXAMPLE OF PATIENT PER ACOUSTIC THERAPY FOR ERPS PRINCIPAL AND INDEPENDENT COMPONENT ANALYSIS

ANNEX AD: EXAMPLE OF PATIENT PER ACOUSTIC THERAPY FOR ERPS SOURCE LOCATION IN 100MS, 200MS, 300MS AND 400MS. 
ANNEX AE: SESSION 4 - SESSION 1 DIFFERENCE OF TIME AND TREQUENCY DOMAIN PARAMETERS FROM EACH GAV ERP ACOUSTIC THERAPY: SELECTION OF PARAMETERS FOR FRONTAL AND TEMPORAL LOBE

ANNEX AF: SESSION 4 - SESSION 1 DIFFERENCE OF TIME AND TREQUENCY DOMAIN PARAMETERS FROM EACH GAV ERP ACOUSTIC THERAPY 


\section{ABSTRACT}

Acoustic therapies have gain relevance due to the sound effects on human body functioning. The ear does not only make humans hear, but it also provides them kinaesthetic sense and significantly modifies the limbic system. Furthermore, to receive and perceive sounds goes beyond to decode and identify auditory stimuli. Sound is the propagation of mechanical waves that cause vibrations in the matter, and which can vibrate at different frequencies. This means that sound may modulate the human oscillation frequencies. It is possible to consider that sound-based treatments could reverse neural synchrony abnormalities in the auditory cortex that are generating tinnitus. Acoustic therapies generally seek to relieve stress and tension caused by tinnitus (i.e., to have a direct effect on the limbic system, which activates the parasympathetic nervous system), reduce tinnitus perception, or redirect the attention, which has been entirely attracted by tinnitus.

To date, a large number of acoustic therapies have been applied to treat tinnitus, with the exception of Binaural beats. The effect that produces those auditory stimuli is, however, not well understood yet. Furthermore, the conventional clinical protocol is based on a trial-error procedure, and there is not a formal and adequate treatment follow-up. At present, the widely used to evaluate acoustic therapies is by means of subjective methods such as Visual Analog Scale (VAS) and ad-hoc questionnaires.

Electroencephalographic (EEG) has become a standard brain imaging tool to quantify and qualify neural oscillations, which are basically spatial, temporal, and spectral patterns associated with particular perceptual, cognitive, motor and emotional processes. Neural oscillations have been traditionally studied on the basis of eventrelated experiments, where time-locked and phase-locked responses (i.e., event-related potentials) along with time-locked but not necessary phase-locked responses (i.e., event-related (de) synchronization) have been essentially estimated. Both potentials and levels of synchronization related to auditory stimuli are herein proposed to assess the effect of acoustic therapies.

In conclusion, it has been previously demonstrated that acoustic therapies have moderate beneficial effects as an alternative treatment of chronic and refractory tinnitus. However, an integral treatment program based on different acoustic therapy designed according to the patient clinical history could increase the sound effects. Moreover, this thesis pursues to establish an objective methodology to treat tinnitus with acoustic therapies based on EEG activity evaluation. 


\section{ACRONYMS AND ABBREVIATIONS}

ACT

ADT

ASSR

BP

Bpm

CHABA

CIA

CIA.EVO

CNS

DWT

EAE

EC

EO

EEG

EAE

ERD/ERS

EROs

ERP

FFT

fMRI

GAv

GP

GUM

HADS

HFT

HR

HRV
Acceptance and Commitment Therapy

Auditory discrimination therapy

Auditory Steady-State Responses

Blood Pressure

Beats per minute

The Committee on Hearing, Bioacoustics, and Biomechanics

Cuestionario Inicial de Acúfenos

Cuestionario Evolución de Acúfenos

Central Nervous System

discrete Wavelet Transform

See TEAE

Eyes-Closed

Eyes-Open

Electroencephalography o Electroencephalogram

See TEAE

Event-Related (De) Synchronization

Event-Related Oscillations

Event-Related Potentials

Fast Fourier Transform

functional Magnetic Resonance Imaging

Grand average ERP GAv

General Practitioner

Guide to Uncertainty in Measurement

Hospital Anxiety and Depression Scale

High Frequency Therapy

Heart Rate

Heart Rate Variability. 


$\begin{array}{ll}\text { ICA } & \begin{array}{l}\text { Independent Component Analysis } \\ \text { INR }\end{array} \\ \begin{array}{l}\text { Instituto Nacional de Rehabilitación (National Institute of } \\ \text { Rehabilitation) }\end{array} \\ \text { I2A2 } & \begin{array}{l}\text { Grupo de Investigación en Instrumentación y Acústica Aplicada } \\ \text { Instituto Tecnológico y de Estudios Superiores de Monterrey, also } \\ \text { known as Tecnológico de Monterrey or simply as Tec }\end{array} \\ \text { MNF } & \text { Mean Frequency } \\ \text { NHS } & \text { UK National Health Service } \\ \text { NIHL } & \text { Noise-Induced Hearing Loss } \\ \text { PCA } & \text { Principal Component Analysis } \\ \text { PET } & \text { Positron Emission Tomography } \\ \text { PSD } & \text { Power Spectral Density } \\ \text { PSQ } & \text { Perceived Stress Questionnaire } \\ \text { RNID } & \text { Royal National Institute for Deaf People } \\ \text { RS } & \text { Relaxing Signal } \\ \text { SFRs } & \text { Spontaneous Firing Rates } \\ \text { SigT } & \text { significant Tinnitus } \\ \text { SoA } & \text { State of the Art } \\ \text { SPL } & \text { Sound Pressure Level } \\ \text { STFT } & \text { Short Time Fourier Transform } \\ \text { TEAE } & \text { Therapy for Enriched Acoustic Environment (also named EAE) } \\ \text { THI } & \text { Tinnitus Handicap Inventory } \\ \text { TMT } & \text { Tinnitus Masking Therapy } \\ \text { TPO } & \text { Tinnitus Phase-Out } \\ \text { TRT } & \text { Tinnitus Retraining Therapy } \\ \text { UPM } & \text { Universidad Politécnica de Madrid } \\ \text { VAS } & \text { Visual Analog Scale } \\ \text { VBM } & \text { Voxel-Based Morphometry } \\ \text { WHO } & \text { World Health Organization } \\ & \end{array}$


wICA

wavelet enhanced ICA method

WT

Wavelet Transform 
"Any man could, if he were so inclined, be the sculptor of his own brain"

Santiago Ramon y Cajal (1852-1934), pathologist, histologist, neuroscientist and Nobel laureate. 


\section{INTRODUCTION}

"In the beginning was Prajapati (Brahman) with whom was the Word; And the Word was verily Brahman"

"I am Om, the Word that is God."

Krishna Yajurveda, Kathaka Samhita, 12.5, 27.1

Bhagavad Gita 7:8 
Music is older than language and even mankind (Ravignani, et al., 2013). It has been used as therapy during thousands of years (see Annex A) before Émile JaquesDalcroze developed a method for learning and experiencing music through movement at the end of 19th century, which was the precursor of music therapy as a medical alternative for the treatment of diseases (Anderson, 2012) or Rauscher (Rauscher, et al., 1993) and later on Campbel (Campbell, 1998) popularized the Mozart effect at the end of the 20th century.

The positive influence of music is a complex subject, conditioned by the structure and functions of the Central Nervous System (CNS) and the neurovegetative system, endocrine glands and internal organs. All this is combined in a complicated foundation, with the musical piece, its melody, harmony, rhythm and timbre, and moreover, the particular psychic disposition of the patient which may create a potential placebo'.

Music therapy is mainly indicated for stress, socialization problems, physical, mental and emotional disorders and serves as a mood regulator (Benenzon, 2004). It triplebalances the individual as biopsychosocial unit (Rodrigo, 2008).

During the last years and with the "New Age" trend, a commercial need has been developed around music therapy and what it means. Anyone is able to buy some of the thousands of CDs to relax, brain develop, creativity empower or against insomnia, depression and quit smoking. On top of this, one person could even download potential acoustic drugs (Tavira Sanchez, 2010).

In order to recognize that the power of music is really effective, a scientific basis is needed (Goldman, 1992). Along the $20^{\text {th }}$ Century, Tomatis and Bernard started to scientifically base their research within ear and voice. The first one created the Tomatis $\AA$ Method (Tomatis, 2017) and the Electronic Ear in order to listen to improve communication. The second one, invented the Eareducator ${ }^{\mathrm{TM}}$ to eliminate the cause of emotional disturbances and get its balance. But there was still a big roof to research leaving aside the "Trial and Error".

To perceive and use any acoustic signal, one should be able to understand among others the body's mechanisms like a doctor, the energy behaviour like a physicist, the behavioural processes like a psychologist, the sound effects on the brain as a psychoacoustic, the hearing mechanisms as an otorhinolaryngologist and the nervous

\footnotetext{
${ }^{1}$ The Latin word placebo means "I will please" and was already used in 1200 being the first word of the first antiphon of Psalm 114, where traditionally God was praised. In the medieval world, placebo meant the power of listening to sacred music, which healed the mind, body and spirit. In 1811, the Hooper's Medical Dictionary defined "placebo" as "any remedy given more to please the patient than to benefit him" (Campbell, 1998).
} 
system and brain as a neurologist (Goldman, 2010), that is why a multidisciplinary team is the key of success ${ }^{2}$.

\subsection{TINNITUS: MEDICAL PROBLEM TO SOLVE}

Tinnitus refers to the perception of sound in absence of an acoustic source (Reavis, et al., 2012; Henry, et al., 2008). It can be unilateral or bilateral. Sounds are usually simple, with common forms resembling pure tones ('ringing'), gaussian noise ('hissing'), or buzzing (Sedley, et al., 2016).

The history of attempts to treat tinnitus date back to ancient Egypt. Assyrian, Roman, Byzantine, Medieval, and Renaissance literature describe too how to classify better this often devastating condition (Heller, 2003).

In 1982, the Committee on Hearing, Bioacoustics and Biomechanics (CHABA) of the United States of America defined tinnitus as a conscious experience of a sound that is originated from the patients' own head. Later, Jastreboff (Jastreboff \& Jastreboff, 2000) redefined the term as the perception of a sound that results exclusively from the activity within the nervous system, and without any corresponding mechanical vibratory activity within the cochlea. In addition, Lenhardt (Lenhardt, 2004) classified tinnitus into objective and subjective. Objective tinnitus (somatosensory sounds) was associated with peripheral vascular abnormalities detectable by stethoscopic inspection, whereas subjective tinnitus was determined as an acoustic perception merely experienced by the patient. In line with acoustic principles, tinnitus is assumed subjective and is defined in terms of intensity and frequency (Kaltenbach, 2006). To characterize tinnitus, it is necessary to construct a frequency response curve, obtained objectively in animals (Noreña \& Eggermont, 2005), but subjectively in human beings (Roberts, et al., 2006).

According to Jastreboff (Jastreboff \& Jastreboff, 2000), people experiencing tinnitus can be grouped into five main categories:

(i) Category 0 , patients without hyperacusis or hearing loss, and whose tinnitus has little impact on their daily lives

(ii) Category 1, patients with a significant tinnitus but without hyperacusis or hearing loss

(iii) Category 2, patients with both significant tinnitus and hearing loss

(iv) Category 3, patients experiencing significant hyperacusis for a long period of time with or without tinnitus

(v) Category 4, patients with progressive tinnitus and hyperacusis.

\footnotetext{
${ }^{2}$ This thesis is under the umbrella of an example of a multidisciplinary team: the neuroacoustic laboratory (http://www.i2a2.upm.es/lab-neuroacustica/) from Grupo de Investigación en Instrumentación y Acústica Aplicada (I2A2) of the Universidad Politécnica de Madrid, Spain (UPM) which investigates not only music but Acoustic therapy. Furthermore, this thesis has been developed with the support of Escuela de Ingeniería y Ciencias from Tecnológico de Monterrey https://tec.mx/es (Campus Mexico City) too.
} 
Another important distinction is between pulsatile and nonpulsatile tinnitus. This is determined by the patient's description of the quality of the sound perception.

- Nonpulsatile tinnitus should be differentiated further into mild and severe types (Sismanis, 1999)

- Mild tinnitus is audible by the patients occasionally or only when in a quiet place and usually is not troublesome.

- Severe tinnitus is a very disturbing symptom, which often degrades the quality of the patient's life.

- Pulsatile tinnitus may be classified further as either vascular or nonvascular in etiology (Sismanis, 1998).

- Nonvascular causes are usually related to myoclonus of the palatal musculature, stapedius muscle, or tensor tympani.

- Vascular etiologies can be differentiated further into venous and arterial.

Clinically, tinnitus is often due to reversible causes, such as exposure to loud noise, fever, ototoxicity or a transient disturbance in the middle ear. (Eggermont \& Roberts, 2004). Its origin is still unknown and there is no medical cure. Tinnitus is generally ignited by hearing loss, and very often by noise-induced hearing loss (NIHL). Some of the tinnitus models are explained in the Annex B. Most chronic tinnitus is of central origin; that is, it is in the brain and not generated in the ear (Eggermont \& Tass, 2015). Head or neck injuries can also trigger the development of tinnitus, as altered somatosensory input can affect auditory pathways and lead to tinnitus. Emotional and attentional state could be involved in the development and maintenance of tinnitus via top-down mechanisms (Shore, et al., 2016).

Tinnitus can be a symptom of various underlying pathologies and be accompanied by many different comorbidities. Therefore, an integrated multidisciplinary approach is needed for comprehensive tinnitus diagnosis and therapeutic steps. A stepwise decision-tree approach for clinical tinnitus management could be used (see Annex C). Basic diagnostic steps are recommended for all patients and should include a detailed case history, assessment of tinnitus severity, clinical ear examination, and audiological measurement of tinnitus and hearing function. For many patients these first diagnostic steps are sufficient for diagnosis, and counselling is sufficient for treatment (Langguth, et al., 2013).

Tinnitus refractory means resistant to a process or stimulus, and it becomes chronic when perceptual inference mechanisms learn to expect tinnitus, engaging connections between auditory and parahippocampal cortex (Sedley, et al., 2016). 


\subsubsection{EPIDEMIOLOGY}

Most of the studies of tinnitus have been done in the Western Europe or USA, and have methodological drawbacks, especially with production of an unambiguous definition of tinnitus. Consequently, the scatter of prevalence estimates is wide, although most study results have shown rates between $10 \%$ and $15 \%$ of the adult population (Heller, 2003; Baguley, et al., 2013; Nondahl, et al., 2010). Also, results of epidemiological studies show similar prevalence in low-income and middle-income countries in Africa and Asia which indicates that the perception of phantom sounds is a global burden (Langguth, et al., 2013). Annex D give more details about the Tinnitus demographics.

\subsubsection{ECONOMIC IMPACT}

Hearing loss has been associated with cognitive decline, dementia, depression, hospitalization, and heart disease, among other diseases and conditions. In U.S. severe to profound hearing loss is expected to cost society $\$ 297.000$ over the lifetime of an individual. Most of these losses (67\%) are due to reduced work productivity, although the use of special education resources among children contributes an additional $21 \%$. Lifetime costs for those with prelingual onset exceed $\$ 1$ million per person (Mohr, et al., 2000).

Tinnitus presents a burden to the health care system that has been rising in recent years. More than the half people (who reports having had tinnitus for five or more continuous minutes in the past year) seek medical attention for tinnitus. Populationbased studies provide crucial underpinning evidence; highlighting the need for further research to address issues around effective diagnosis and clinical management of this heterogeneous condition (Martinez, et al., 2015).

Tinnitus is a symptom that is associated with practically every known ear disorder and so if a patient complains of the condition, it is appropriate to examine the range of possible otological etiologies.

Martinez and his colleagues (Martinez, et al., 2015) calculated that the yearly cost of tinnitus to the UK National Health Service (NHS) in England could easily exceed $£ 4.9$ million, without taking into consideration the socioeconomic aspect. This estimate alone is based on each incident case in 2011 where one General Practitioner (GP) appointment is $£ 45$ (PSSRU, 2013) and one outpatient appointment is £108 (NHS, 2014), knowing that on average GPs in England typically refer only $37 \%$ of their tinnitus patients (El-Shunnar, et al., 2011). If any additional prescriptions, diagnostic assessments, procedures, or referrals are required, then this would of course markedly elevate the health economic costs.

The team of Maes (Maes, et al., 2013) did a cost study for the Tinnitus in the Netherlands as well. Total mean societal cost of illness was $€ 6.8$ billion. The larger part 
of total cost of illness was not related to health care (average absent from the job was 15,41 days). Total mean health care costs were $€ 1.9$ billion ( $€ 1.541$ per patient visiting GP, specialist, other professional and medication). For the $11 \%$ of patients, a maximum of $€ 6.832$ (per person) was expended as "out-of-pocket" costs like food supplements, alternative therapies, headphones, ear protection or sound-isolation materials for walls, floors and ceilings. Significant predictors of both health care costs and societal costs were tinnitus severity, age, shorter duration of tinnitus, and more severe depression.

On top of that, for those patients who need hearing aids, cochlear implants or other hearing devices, the costs of acquiring and maintaining such a device remain prohibitive for most potential users in low (and middle) income countries (McPherson, 2011; Baltussen, et al., 2009; Bond, et al., 2009 ; Peñaranda, et al., 2012). According to WHO, the target price for an "affordable" hearing aid should be no more than $3 \%$ of the per capita of the user's country (McPherson, 2011). Hearing aids can range from $\$ 1.000$ on up to more than $\$ 4.000$ for each device depending on the level of technology (Dybala, 2016). In addition, they exclude the costs of ear moulds, maintenance and the periodic purchase of batteries. The scenario is even more daunting for cochlear implants, which are associated with an estimated lifetime cost of about $\$ 90.000$ per child with severe to profound hearing impairment (Saunders \& Barrs, 2011; Kral \& O'Donoghue, 2010 ).

\subsection{STATE OF THE ART}

Generally speaking, sound therapy is the appropriate and conscious use of sound energy to promote well-being in humans and also the expansion of consciousness. The therapy through sounds is based on the knowledge that all matter is vibrating at specific frequencies. These therapies can influence physiological, cognitive and even psychological variables (Koelsch, 2009).

Nowadays there is a wide variety of treatments for tinnitus, including hearing aids, masking therapy, counselling, retraining therapy, music therapy, acupuncture, herbal treatments and acoustic therapy (Hanley, et al., 2008), none of them $100 \%$ effective (Sweetow \& Sabes, 2010).

Audiologists have always known that sounds could interfere with tinnitus (Saltzman \& Ersner, 1947; Surr, et al., 1985; Surr, et al., 2002; Melin, et al., 1987), though there is no consensus on how to measure the effectiveness of the outcomes of tinnitus treatments so that they remain inconclusive (Meikle, et al., 2008; Kamalski, et al., 2010; Hoare, et al., 2012).

Acoustic therapies aim to reverse the neuroplasticity phenomenon related to tinnitus by adequately stimulating the auditory pathway. If neuro-plastic changes were produced, habituation and/or residual inhibition of the tinnitus could be achieved (Formby \& 
Scherer, 2013). Habituation refers to the elimination of the tinnitus effects without the elimination of the sound perception per se. In contrast, residual inhibition is the reduction or disappearance of the tinnitus perception. The inhibition might last from a few seconds to days. Some of the most relevant acoustic therapies so far are the following:

- Tinnitus masking therapy (TMT) aims to remove the tinnitus perception by using a supportive auditory stimulus (masker). A wide frequency band is used as a masker, and for many patients, the ipsilateral masking is as effective as the contralateral, suggesting effectiveness of the therapy at a cortical level (Lenhardt, 2004).

- Tinnitus retraining therapy (TRT) is a combination of counselling and sound therapy based on neutral low level broad-band noise. It aims to habituate the patient to the tinnitus. This therapy has two main objectives: to get used to the reactions of limbic and autonomic systems, as well as to the tinnitus perception. TRT can be effective, regardless of the etiology of tinnitus (Jastreboff \& Jastreboff, 2000).

- Tinnitus phase-out (TPO) is based on the theory of active noise control, which refers to the mutual cancellation of two noise signals with opposite phase (Hansen \& Snyder, 1997). When a signal (primary noise) is mixed with the same one but in reverse phase (secondary noise), the resulting residual noise is highly attenuated (Choy, 2004; Choy, 2007). The major challenge for this method obtaining the acoustic features of the tinnitus.

- High frequency therapy (HFT) stimulates the auditory system through stimuli adjusted according to the hearing loss curve of the patient. As most patients have hearing losses at high frequencies, piezoelectric devices have been designed to generate acoustic signals between 6 and $20 \mathrm{kHz}$, to be placed on the mastoid as audiometry transducers (Goldstein, et al., 2001; Goldstein, et al., 2005). These devices stimulate the auditory cortical neurons, rather than the external auditory system.

- Auditory discrimination therapy (ADT) strengthens auditory cortical areas that respond to training frequencies, and attenuates the tinnitus pitch perception (Herraiz, et al., 2007; Flor, et al., 2004).

- Therapy for enriched acoustic environment (TEAE) uses burst sequences or pip tones that stimulate the auditory pathway selectively and personally (Powers \& dos Santos, 2015). On one side, each tone sequence has a response similar to neural tuning curves of the auditory pathway frequencies. On the other side, the stimulus is tuned to the patient hearing loss curve. 
- Neuromodulation therapy attempts to desynchronize the neural networks working abnormally, under the hypothesis that tinnitus results from a pathological neural synchronization (Adamchic, et al., 2014; Hauptmann, et al., 2015).

- Binaural beats therapy consists of two different pure-tones sine waves at frequencies below $1.5 \mathrm{kHz}$ and with a frequency rate lower than $40 \mathrm{~Hz}$ presented to each ear, where an amplitude-modulated signal is created with a beat of the different of both frequency waves (Draganova, et al., 2008). See Annex E for more info.

Electrophysiological (Eggermont \& Roberts, 2004) and functional imaging measurements suggest (but not limit to) the following neural correlates of tinnitus in the auditory system: increased neural synchrony, reorganization of tonotopic maps and increased spontaneous firing rates (SFRs) in the auditory system. Tinnitus is likely the result of maladaptive plasticity of the CNS. The CNS wants to restore its evoked neural activity levels that had been lowered by the hearing loss. This is done by increasing the efficacy (or gain) of its synapses. But this gain also affects the SFR, which occurs in the absence of a physical sound source, and will then generally increase (Schaette \& Kempter, 2006).

Specifically, electrophysiological studies have revealed that the brain oscillatory activity decreases in the alpha band $(10-14 \mathrm{~Hz})$, and increases in delta $(1.5-4 \mathrm{~Hz})$ and gamma ( $30 \mathrm{~Hz}$ ) bands (Powers \& dos Santos, 2015; Adamchic, et al., 2014). Abnormal brain oscillations on the frontal lobe have also been found. As the frontal lobe is related to the emotional and attentional regulation, the frontal deficiency is attributed to the stress caused by tinnitus (Adamchic, et al., 2014). On the other hand, several functional brain imaging studies shows aberrant neural activity within the central auditory pathway of tinnitus patients. In detail, changes in the inferior colliculus, the thalamus and the auditory cortex have been demonstrated by using both Positron Emission Tomography (PET) (Arnold, et al., 1996; Cacace, et al., 1999; Eichhammer, et al., 2003; Giraud, et al., 1999; Langguth, et al., 2006; Lockwood, et al., 1998; Mirz, et al., 1999; Reyes, et al., 2002; Wang, et al., 2001) and functional Magnetic Resonance Imaging (fMRI) (Lanting, et al., 2008; Melcher, et al., 2000; Smits, et al., 2007). Alterations of neural activity were also observed in non-auditory brain structures, especially in the limbic system (Andersson, et al., 2000; Lockwood, et al., 1998; Mirz, et al., 2000). Using highresolution magnetic resonance imaging and Voxel-Based Morphometry (VBM), circumscribed alterations in the auditory system (medial geniculate nucleus of the thalamus) and the limbic system (i.e. the subcallosal region including the nucleus accumbens) were detected (Muhlau, et al., 2006). 


\subsubsection{GAPS AND UNKNOWING}

After reviewing some of the most applied and researched acoustic therapies from the last years for Tinnitus, there were some lack of knowledge and gaps in the results:

- exact mechanisms of the auditory entrainment should be investigated by means of respective neurophysiological methods (MEG/EEG) to test if and how auditory entrainment and possibly related tinnitus suppression is reflected by neural oscillations (Herraiz, et al., 2007; Ito , et al., 2009; Lee , et al., 2017; Herraiz, et al., 2007; Neff, et al., 2017; David, et al., 2010; Hauptmann, et al., 2017; Williams, et al., 2015; Herraiz, et al., 2010).

- few acoustic therapies didn't explain any effect in normal audiogram

- hearing loss showed no differences by Tinnitus Handicap Inventory (THI) scores or other questionnaires

o there were no objective inputs to correlate with

- some studies did not measure subjective inputs (for example trough tinnitus questionnaires) or not correlate EEG or MEG data with tinnitus sufferers' clinical features. Therefore, an additional validation is needed to establish the value of any customizing therapeutic approach (Gerken, et al., 2001; Diesch, et al., 2010; Lee, et al., 2007 ; Houdayer, et al., 2015).

- there was a lack of a control group in order to compare the same questionnaires and test with the tinnitus patients, and consequently, it is difficult to reach an absolute conclusion regarding to what extent the observed effects are related directly to the acoustic therapies (David, et al., 2010; Lee , et al., 2017; Neff, et al., 2017; Williams, et al., 2015)

- a strong limitation of the studies exists due to the relatively small samples (Hauptmann, et al., 2017; Adamchic, et al., 2014; Vanneste, et al., 2013)

- if the observed patient groups were made up of paying clients it is unknown as to whether this could have caused any additional placebo effects to influence the final results (Williams, et al., 2015).

- there was a high variety of tinnitus cases and non-standardized participant selection criteria that makes a common explanation less likely (Pierzycki, et al., 2016; Vanneste, et al., 2013), such as

- Tinnitus may be the result of cochlear trauma, or might be related to temporomandibular dysfunction or whiplash.

o The fact that patients are only selected if the tinnitus pitch fell within an area of hearing loss.

- subjects who might be chronic (more than 3 months) or also may have some hyperacusis

- a non-standardized MEG or EEG data acquisition and data analysis protocol should be required (Pierzycki, et al., 2016) 
- Longitudinal studies in real life should be performed to evaluate the envisioned long-term goal of the approach, namely to develop individually-customized mobile tinnitus acoustic therapies with aesthetically appealing sounds (Neff, et al., 2017).

\subsection{HYPOTHESIS, GENERAL AND SPECIFIC OBJECTIVES}

\subsubsection{HYPOTHESIS}

Many acoustic therapies have been designed to treat tinnitus; however, the cause of the corresponding auditory stimulus is not yet well understood. Up to now, the effectiveness of the acoustic therapies to treat patients suffering from tinnitus is evaluated by means of a visual analog scale and/or ad hoc questionnaires. The visual analog scale is used to quantify subjectively certain sensations of the patients such as pain. This scale is a line with one end representing lack of sensation and the other, extreme feeling. Patients mark a point on the line that matches their sensation magnitude. On the other hand, the questionnaires consist of a series of questions to identify the difficulties that patients are facing due to the tinnitus, and whether those difficulties are overcome after applying an acoustic therapy. Typically, only three responses are provided in the questionnaires: yes, sometimes, and no (Powers \& dos Santos, 2015; Adamchic, et al., 2014). Despite the popularity of these two methods, the resulting evaluation is completely subjective and does not allow an effective quantification of the acoustic therapy effects. This has leaded to apply the acoustic therapies following a trial-error procedure, what delays the patient healing, or even could deteriorate the patient condition.

As the goal of the acoustic therapies is the habituation of reaction of the tinnitus or suppression of the tinnitus as an evaluation method, it is hypothesized that these therapies should produce perceptual and cognitive changes (specifically those related to attention and memory), even though they could not be beneficial.

\subsubsection{OBJECTIVES}

As very little attention has been paid to the importance of an objective method to evaluate acoustic therapies used to treat tinnitus (Adamchic, et al., 2014; Hauptmann, et al., 2015), this thesis seeks to establish a new methodology, based on the monitoring of the treatment at a cortical level. A likely cause of tinnitus is the neuronal hyperactivity in the auditory nervous system, therefore, electroencephalography (EEG), which measures non-invasively the electrical activity of the cerebral cortex, seems to be a feasible method to assess objectively the effects of an acoustic therapy. 


\subsubsection{General objectives}

This study has two primary aims:

1. To apply a Relaxing Signal (RS) and three (TRT, TEAE and binaural) of the eight previously described acoustic therapies to patients suffering from tinnitus, who had received other palliative treatments with no positive results;

2. To record the EEG signals of the patients suffering from tinnitus in order to get objective data to correlate them with this subjective problem. It will be done before, during and after the application of the corresponding acoustic therapy, so as to analyse the neural behaviour of auditory and non-auditory nervous systems. The study of spontaneous activity at resting state, evoked activity and induced activity to be undertaken after data collection.

Moreover, it is expected that the decoding (temporal lobe) and interpretation (frontal lobe) of the auditory stimulus used in the acoustic therapy should be different before and after the therapy. On the basis of perceptual and cognitive changes, the selection and duration of the acoustic therapy for each patient can become objective and much more effective.

\subsubsection{Specific objectives}

- Review the state of the art of application of acoustic therapies for Tinnitus.

- Theoretical study: design of a protocol to evaluate acoustic therapies for the treatment of Tinnitus

- Experimental study:

- Generation of signals as acoustic therapies, measure and analysis of subjective and objective patient's data.

- Evaluate electrophysiological changes of brain activity observed before and after the acoustic stimulation.

- Final outcome:

- Make sure acoustic therapies are effective

- Establish what acoustic therapy is more suitable for each patient and find the objective way for matching variety of patients with acoustic therapies.

- Improve the quality of life of patients with chronic and refractory Tinnitus 


\section{MATERIALS AND METHODS}

There is geometry in the humming of the strings, there is music in the spacing of the spheres.

Pythagoras of Samos (circa 570 - circa 495 B.C.), Greek philosopher and mathematician 


\subsection{INTRODUCTION}

In order to establish a methodology to follow this thesis, there were two previous researches within the I2A2 neuroacoustic department of UPM. The first one related with 78 healthy adults to test the acoustic therapy protocol using binaural beats (Crespo Pelayo, et al., 2013) and the second one focus on Parkinson patients (Galvez Garcia, 2015)

On the other hand, this project has been supported by collaboration between I2A2, Monterrey Institute of Technology and Higher Education (ITESM) and the National Institute of Rehabilitation $\left(\mathrm{INR}^{3}\right)$.

\subsection{SAMPLE}

A random sample of patients from the INR suffering refractory and chronic tinnitus, who had been received allopathic treatments with no positive effects, was asked to take part voluntarily in the present project. They were informed about the experimental procedure, and notified that their head physician was also following-up the project.

There were recruited 68 patients between 37 and 71 years old where the $63 \%$ where women. All of them without any history of otitis, cerebellopontine angle tumours, psychiatrist pathologies, demyelinating diseases of the nervous system, or epilepsy. As it was really difficult to obtain that number of patients due to the dependency of an institution or hospital and the specific state of the Tinnitus, patients with normal audition, unilateral or bilateral hyperacusis, and/or conductive sensory-neural hyperacusis were included too.

Jointly with the patient information, it has been received the audiology evaluation carried out by the INR. Such evaluation included audiometry, hearing loss, tinnitus sound pitch (i.e., acufenometry / perception of tinnitus), minimum masking level (i.e., volume at which an external narrowband noise masks or covers), loudness discomfort level (i.e., volume at which external sound becomes uncomfortable or painful for a tinnitus patient) and Tinnitus Handicap Inventory (THI).

None of the tinnitus patients received any money and they received an audio player without any additional charges fee.

The following figures 1, 2, 3 and 4 show the percentage of patients where the tinnitus was located, the hearing loss level and the number of patients with the different tinnitus frequency and intensity tones.

\footnotetext{
${ }^{3}$ Instituto Nacional de Rehabilitación http://www.inr.gob.mx/
} 


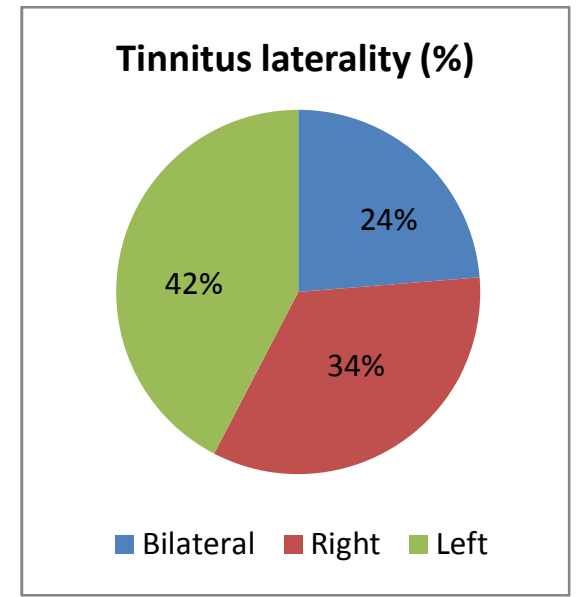

Figure 1: \% of tinnitus laterality from the patients

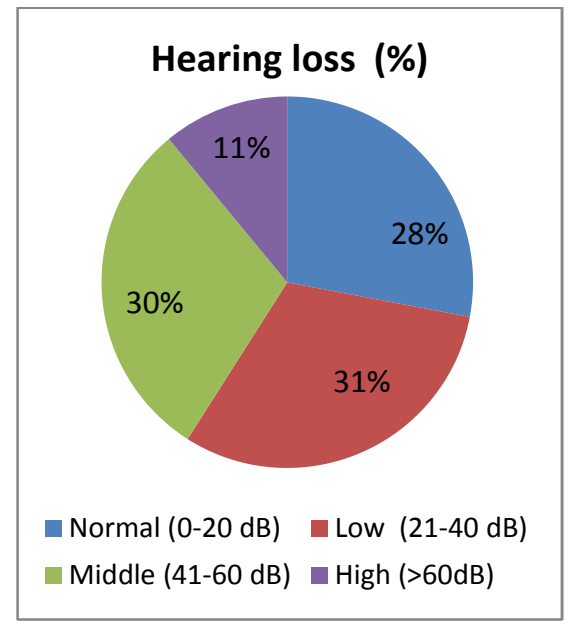

Figure 3: \% of hearing loss level from the patients

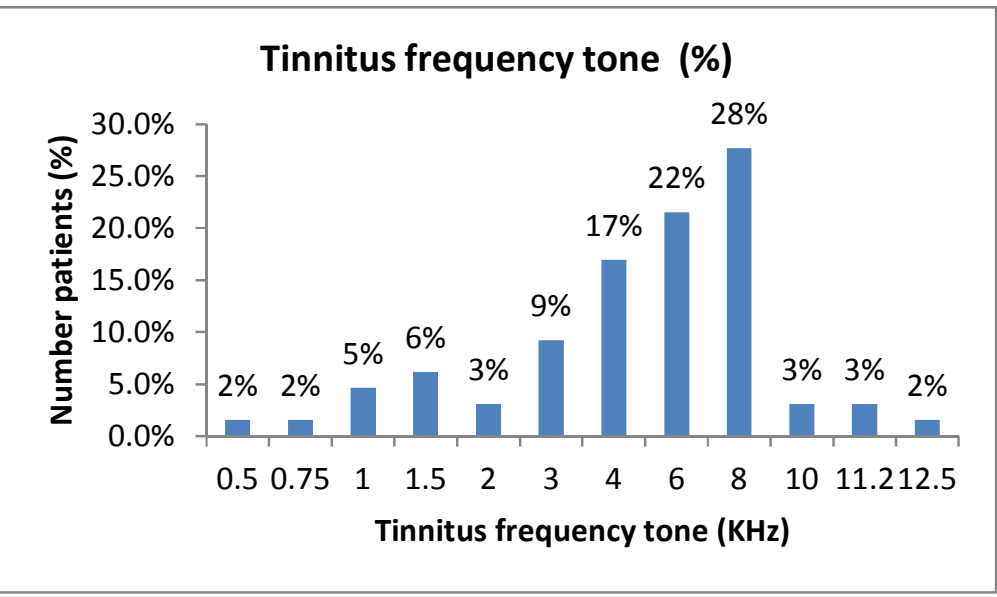

Figure 2: \% of patients with different tinnitus frequency tones

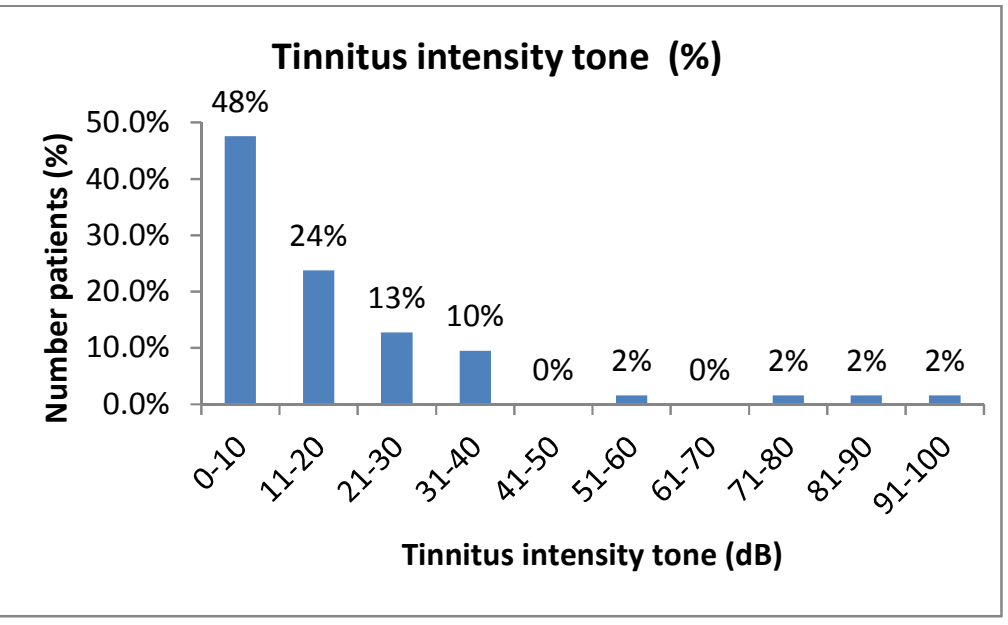

Figure 4: \% of patients with different tinnitus intensity

\subsection{GENERATION OF THE ACOUSTIC THERAPIES}

In order to generate the acoustic therapies, two main software programs were used: MATLAB and Audacity.

- MATLAB is a proprietary programming language developed by MathWorks.

- Audacity ${ }^{4}$ is free open-source audio software for multi-track recording and editing.

The acoustic therapies were designed in line with the patient audiology evaluation carried out by the INR. The main adjustment parameters are taken from the audiometry,

\footnotetext{
${ }^{4}$ Audacity is available at http://www.audacityteam.org/ 
which measures the ability of each ear to perceive the vibrations within different frequency bands of the audible spectrum and Tinnitus sound pitch.

Three acoustic therapies described in section "1.2 State of the Art" were considered besides of the music for the RS group. They were chosen because

- they have been demonstrated better results than others (TRT or TEAE) in previous work (Hanley, et al., 2008; Formby \& Scherer, 2013)

- there were not many references (binaural beats) for tinnitus but for reducing stress and anxiety (Norhazman, et al., 2014; Young, et al., 2014; Weiland, et al., 2011) with good results and therefore they could affect directly in the alpha and beta bands.

Patients were submitted to a random selection so as to belong to a therapy group or a RS group. The essential features of the acoustic therapies under study are the following (Vernon, et al., 2014; Chaieb, et al., 2015; Reedijk, et al., 2015; Reedijk, et al., 2013; Durrant \& Lovrinic, 1995; Howard \& Angus, 2009; Oster, 1973; Kasprzak , 2011):

- Relaxing Signal (RS): For this group, a simple commercial soft signal is used ${ }^{5}$. It could be identified as new age, electronic, downtempo (50 to $90 \mathrm{bpm}$ ) or ambient music.

- Tinnitus Retraining Therapy (TRT): To generate this therapy, a random noise signal is used. This signal is additionally filtered by octave bands, depending on the tinnitus frequencies and hearing loss in each ear. TRT is achieved by directive counselling and exposure to low-level broadband noise. The first component of TRT, directive counselling, may change the way tinnitus is perceived. The patient is taught the basic knowledge about the auditory system and its function, the mechanism of tinnitus generation and the annoyance associated with tinnitus. The repetition of these points in the follow-ups helps the patient to perceive the signal as a non-danger. The second element of TRT therapy, sound therapy, aims to decrease the sound contrast between tinnitus and silent environment leading to a reduced detection of tinnitus. A sample test of TRT signal (filtered from $1 \mathrm{KHz}$ to $4 \mathrm{KHz}$ ) is shown in the Annex $\mathrm{F}$.

- Therapy for Enriched Acoustic Environment (TEAE): This therapy is based on a sequence of random frequency tones (burst and pip pulses) with amplitude proportional to the hearing lost reported on patients' audiometry. Frequency pulses stimulate the auditory pathway in a selective and personalized way. The stimulation is selective, because each tone of the sequence has a response curve in frequencies very similar to the curves of tuning neurons of the auditory pathway. The stimulation is personalized and enriched, because it is designed

\footnotetext{
${ }^{5}$ Surya (11) - Chakra-Heilung II / Chakra Healing II https://www.discogs.com/Surya-Chakra-Heilung-IIChakra-Healing-II/release/7353850
} 
based on the hearing loss of each patient. A sample test of TEAE signal is shown in the Annex F.

- Binaural beats Therapy: This therapy is the one with more evidence of psychoacoustic effects from all the other therapies (see Annex $E$ for more info). $A$ sample test of binaural signal is shown in the Annex $F$. As explained previously, it consists of two different pure-tones sine waves at frequencies below $1.5 \mathrm{kHz}$ and with a frequency rate lower than $40 \mathrm{~Hz}$. There is a very little and contradictory scientific rigor in the definition of these parameters and it has been selected the following:

- Frequency rate $10 \mathrm{~Hz}$ linked to previous studies for reducing stress or anxiety (see Annex E).

- Carrier signal: tinnitus frequency or "commercial" health frequencies.

- When a patient had a tinnitus frequency less than $1.5 \mathrm{KHz}$, it was selected

- Following the recommendations from Vernon and his colleagues (2014) of choosing a carrier signal around $400 \mathrm{~Hz}$, it was selected two "popular" frequencies named "health frequencies"

- $396 \mathrm{~Hz}$ : It is linked with "Ut" (Do) from the ancient solfeggio scale (Boyd \& Elprehzleinn, 2016) or first chakra (muladhara) frequency (Smith, 2017)

- $432 \mathrm{~Hz}$ : It is linked with Verdi and Mozart, mathematically consistent with the Fibonacci series of numbers (apedh, 2014) or the "Real da Vinci Code" (Horowitz, 2007) and with the instrument of Orpheus, the god of music within the archaic Greek Eleusinian Mysteries (Collins, 2017; Horowitz, 2015).

- No pink noise in order to avoid any possible influence or therapeutic effect (Lindberg, et al., 1987; Huotilainen, et al., 2015; Drexler, et al., 2016)

\subsection{FACILITIES}

\subsubsection{LABORATORY}

Patients were examined in a well-equipped research laboratory (Figure 5) at Tecnológico de Monterrey (ITESM), in the Campus Mexico City. It had the appropriate conditions to attend people, and record EEG data. Those conditions included a quiet atmosphere, water and toilet services, washing facilities, quiet air conditioner and parking place. 


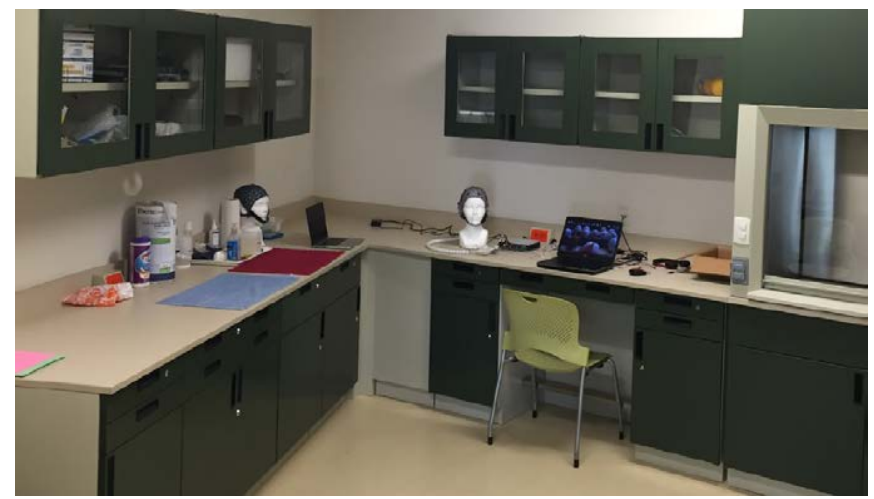

Figure 5: Available laboratory at ITESM, where the measurements were taken ${ }^{6}$

\subsubsection{EQUIPMENT AND SOFTWARE}

To record the EEG data, a g.USBamp (Figure 6a) was available at the laboratory. The g.USBamp is a high-performance and high-accuracy biosignal amplifier. It allows acquiring sixteen EEG channels at a sampling frequency of up to $32.7 \mathrm{kHz}$. The amplifier is configured to sample at $256 \mathrm{~Hz}$ within a bandwidth between 0.1 and $100 \mathrm{~Hz}$ (covering from delta to gamma) and a low pass Butterworth filter in $60 \mathrm{~Hz}(58-62 \mathrm{~Hz})^{7}$. Seventeen of the nineteen recording sites of the 10/20 system were employed following standard guidelines (Pivik, et al., 1993). The Cz channel was used for referencing the other sixteen EEG channels, and left lobe ear worked as ground. The EEG configuration is illustrated in Figure 6b.

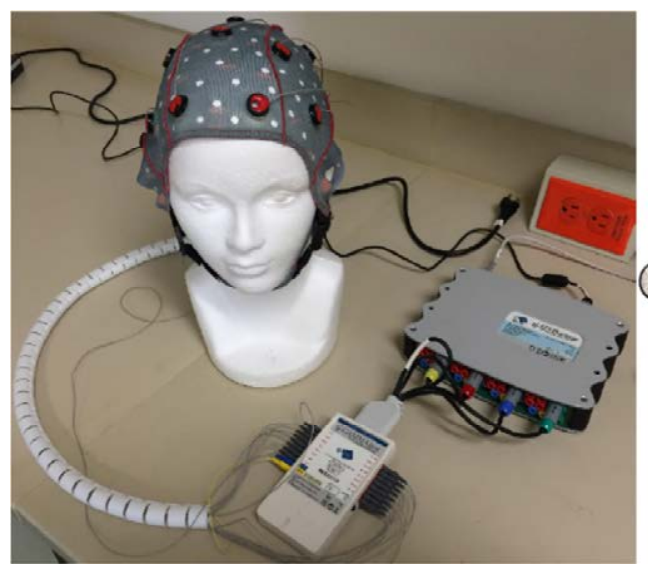

(a)

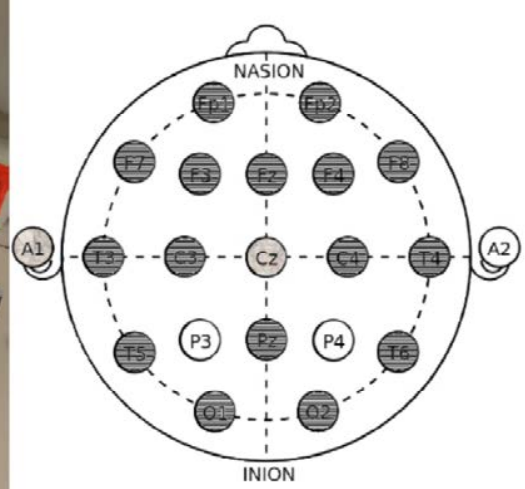

(b)

Figure 6: (a) the equipment employed to record the EEG signals; (b) the 17 EEG channels based on the 10/20 system which were used. Only channels P3 and P4 could not be recorded because of infrastructure limitation

\footnotetext{
${ }^{6}$ This laboratory was chosen because of the low background noise, which was measured before testing and was around $35 \mathrm{dBA}$. This parameter is good enough to apply the acoustic therapies and to record EEG data.

${ }^{7}$ In Mexico the electricity is 127 Volts alternating at 60 cycles per second
} 
To set-up the experimental procedure, OpenViBE ${ }^{8}$ software was used. OpenViBE is open-source multi-platform software for brain-computer interfaces and real-time Neurosciences. This software is a very feasible solution since it has an easy to use graphical language, and it also provides a compatible acquisition server with g.USBamp.

\subsubsection{DEVICES FOR ACOUSTIC THERAPIES}

Audio players are provided to patients. These audio players have a 4Gb memory, and include a pair of headphones and a battery charger. Annex $G$ shows the player details.

\subsubsection{Distortion}

Due to the audio chain (player + headphones), it is possible that the acoustic signal that the patient receives through headphones, loses fidelity given the physical and electronic properties of the instruments involved in the audio chain. Therefore, it is necessary to control the audio properties of the sound in the laboratory with the proper equipment. In order to evaluate the audio quality, the audio chain is composed by acoustic signal, audio player, analyser, headphones and Sound Pressure Level (SPL), as explained in the Annex G.

In order to know the error between the design of the acoustic therapy and its application, it was necessary to verify the signal along to the audio chain, and two tests were carried out.

1. The output of audio player was connected to the input of the analyser and the signal was recorded. Annex $G$ shows the frequency response of a selected frequency. As it can be seen, there is either no distortion or other kind of impurities in the original signal.

2. The sound pressure emitted by the headphones was measured by a SPL Meter as a flat wave front, and its frequency response was recorded with a Fast Fourier Transform analyser (see Annex G). There is no distortion in the signal and only a slight power increase between 3 and $4 \mathrm{kHz}$ due to intrinsic components of the headphones and the equalization was taken into account in order to correct the frequency response of the headphones. This frequency response of the headphones is sufficiently good to be used for acoustic therapy purposes.

In addition, the volume limit was established in the device according to the audiometry curve of each patient.

\footnotetext{
${ }^{8}$ OpenViBE is available at http://openvibe.inria.fr/
} 


\subsubsection{Calibration and Uncertainty}

As explained in the Annex $\mathrm{H}$, the extended uncertainty is calculated mainly from the following uncertainties

- Type A: repeatability occurred on the audio player and the system headphones / SPL

- Type B: SPL calibration and headphone spatial position

And also calculated in the $\underline{\text { Annex } H}$, it could be seen that the extended uncertainty $(U)$ is approximately $3,68 \mathrm{~dB}$

\subsection{ELECTROENCEPHALOGRAPHIC EVALUATION}

EEG is a non-invasive and relatively non-expensive technique that measures the electric fields produced by the pyramidal neurons of the cerebral cortex during the synaptic communication. The synapse is the communication bridge between the axon (output) of a neuron and dendrites (inputs) of the next neuron. The number of synaptic inputs (dendrites) that are simultaneously excited determines the amplitude of the EEG signals. EEG signals are also characterized by the frequency at which oscillates the neural networks, and it can range between 0.01 and $600 \mathrm{~Hz}$. In general, perception, cognitive states, motor activity and emotional processes determine the oscillation frequency of the neural networks (DeMarre \& Michael, 1983). EEG is an extensively used method that gives an insight into the complex and dynamic mechanisms of the human brain: from the decoding of external stimuli registered by the sense organs, to the programming of a reaction executed through the muscles (Kropotov, 2009).

Brain responses have been traditionally studied on the basis of event-related experiments, where time-locked and phase-locked responses along with time-locked but not necessary phase-locked have been essentially analysed (Adjamian, 2014; Cohen, 2017).

In tinnitus research, neuroimaging techniques have been used to find the association of this neurological disease with alterations in different brain areas. Neural systems related to attention, perception, distress, memory and emotions have been of special interest. EEG findings are particularly discussed hereinafter, along with their relevance for the present thesis.

Traditionally, EEG signals have been studied in three directions: spontaneous activity, evoked activity, and induced activity.

\subsubsection{SPONTANEOUS ACTIVITY AT RESTING STATE}

Several theories on the neural anomalies due to tinnitus have been proposed. Most of investigators have opted to examine tinnitus effects at resting state, since the human 
brain generates spontaneous fluctuations in widely separated brain regions, and within a wide frequency range $(0-100 \mathrm{~Hz})$.

Traditionally, neurophysiological effects of tinnitus have been assessed under the following conditions (Hong, et al., 2016; Elgoyhen, et al., 2015; Vanneste, et al., 2014; Balkenhol, et al., 2013; Meyer, et al., 2014; Pierzycki, et al., 2016; Houdayer, et al., 2015; Vanneste, et al., 2013):

- comparison of brain activity between tinnitus and non-tinnitus participants

- experiments undertaken in a quiet room

- participants in seated position in most of the cases

- EEG montage with different number of electrodes, from 19 to 128 recording sites

- $\quad$ eyes-close (EC), eyes-open (EO), and thereof conditions have been considered;

- EEG recordings from 2 to 10 minutes long have been taken

- power and amplitudes of EEG signals across delta $\delta(0.1-4 \mathrm{~Hz})$, theta $\theta(4-8 \mathrm{~Hz})$, alpha $\alpha(8-13 \mathrm{~Hz})$, beta $\beta(13-30 \mathrm{~Hz})$ and gamma $\gamma(>30 \mathrm{~Hz})$ frequency bands have been particularly explored.

One of the main finding of aforementioned studies have provided neurophysiological and neuro-dynamic evidence to show that tinnitus is due to both bottom-up and topdown dysfunctions. This means that not only peripheral auditory system, but central non-auditory cognitive system is also involved in tinnitus genesis (Hong, et al., 2016). However, there is still controversy about the association of chronic tinnitus and dysrhythmias of thalamus-cortical circuits that modulated spontaneous brain oscillations. In the Annex I is shown a summary of the most remarkable differences in ongoing brain oscillations that had been quantified across tinnitus and control groups.

Despite important advances, there are many other signal-processing tools that can be applied on spontaneous activity to reveal tinnitus nature (see Annex J), including relative power, coherence, time-frequency analysis, and entropy (see Annex K).

\subsubsection{EVOKED ACTIVITY: EVENT-RELATED POTENTIALS (ERPs)}

The traditional way to measure evoked activity is via Event-related potentials (ERPs). These appear after the onset of a sensory, cognitive and motor stimulus, and are phase-and time-locked to it. ERPs seem to have perceptual significance and be affected by cognitive factors (Niedermeyer \& Lopes da Silva, 2005; Luck, 2005; Schabus, 2001; Houdayer, et al., 2015). It seems that the amplitude of these evoked responses depends on the action potentials of post-synaptic neural connections, and the number of neurons engaged in the stimulus processing.

They are of two types (Picton \& Fitzgerald, 1983; Proverbio \& Zani, 2002; HernádezCordero, 2015): 
- Exogenous components appear within 100ms after stimulus onset, and are defined by the physical characteristics of such stimulus (e.g., intensity, tone, frequency, pitch and timbre).

- Endogenous components depend on psychological variables such as attention or task relevance.

In particular, auditory ERPs are originated from primary cortical areas and depend on the cognitive state of the patient. Some typical auditory ERPs are P1, N1, P2 and N2, which respectively occur 50,100, 170 and 250ms after stimulus onset (HernádezCordero, 2015). See Annex J for info.

\subsubsection{INDUCED ACTIVITY}

In contrast to evoked activity, induce response refers to modulations of ongoing neural activity commonly quantified by event-related oscillations (EROs). As EROs reflect the coupling and uncoupling of neural networks, these EEG parameter gives an insight into the functional neural network dynamics (Bastiaansen, et al., 2012). There are many approaches to study EROs, including event-related band power, event-related spectrum, single-trial Wavelet, multi-taper analysis, event-related coherence, and phase-locking statistics (Annex J $)$. As far as it is known, none of these approaches have been undertaken to investigate electrophysiological anomalies of tinnitus suffers. Based on previous clinical evidence, it is considered that they could be a valuable tool. By way of illustration, it is briefly commented on the principle and the clinical applications of the most widely used method: event-related (de) synchronization (see Annex L).

\subsubsection{OTHER EEG MEASURES}

Apart from the typical EEG parameters such as frequency, latency, magnitude, and scalp topography, other measures should be considered. As a case in point, the phase of the neural oscillations in different brain areas seems to allow studying network communication and information transmission regulation. Another case in point is the source localization technique, which has been proposed to identify responsible neural generators. Cross-frequency-coupling is other potential EEG measure that should be considered since it reflects the coordination of neural dynamics across temporal and spatial scales (Peng \& Tang, 2016).

\subsubsection{ANALYSIS STRATEGY}

The existing body of research concerning neuroimaging studies on tinnitus has attempted to validate EEG measures as a biomarker to diagnose tinnitus and monitor its progress. Although no validation has been achieved yet, analysis of spontaneous and evoked activity has revealed valuable information. In the case of spontaneous activity examination, modulation of brain rhythms in delta, theta, alpha, beta and gamma frequency bands has been associated with chronic dysrhythmias of thalamus cortical 
circuits, tinnitus loudness and perception, level of attention, and intensity of destructive emotions. Newly research conducted by Pierzycki and his colleagues in 2016, found no association between psychoacoustic and psychosocial scores and brain oscillatory activity at the time to assess tinnitus perception. They concluded that EEG rhythms should not be considered as a viable biomarker or outcome measure in clinical trial of tinnitus (Pierzycki, et al., 2016). However, further improvements on analysis procedures followed up to now could lead to obtain more concrete results. This can be illustrated briefly by the work of Klimesch. He considered that theoretical EEG frequency bands were not appropriate since they vary according to age, sex, and current mental state (Klimesch, 1999).

Furthermore, he recently proposed a new method to calculate real frequency bands in accordance with heart rate (Klimesch, 2013). Therefore, the calculation of individual EEG frequency bands for each patient was permitting a more properly spontaneous activity examination in our study. Regarding evoked activity analysis, the EEG waveform P1-N1-P2 has been the most studied. The three components are maximal on fronto-central regions and are involved in auditory stimulus processing, attention mechanisms, and memory processes. The vast majority of studies on ERPs (HernádezCordero, 2015; Attias, et al., 1995; Attias, et al., 1996; Attias, et al., 1993; Delb, et al., 2008; Walpurger, et al., 2003) have found that patients suffering from tinnitus have generally shorter and later components than healthy people, perhaps owing to deficiencies in the central auditory systems. Recently (Adjamian, 2014), it has been questioned the validity of ERP-assessment since the majority of studies only use $1 \mathrm{kHz}$ tones. These tones have been preferred because they evoke optimal auditory ERPs, but there is no relevance between the auditory stimulus and tinnitus. The selection of relevant auditory stimulus to evoke EEG activity in order to investigate the cortical reorganization due to tinnitus evolution is discussed in Section "2.6 Procedure".

Apart from the examination of spontaneous and evoked EEG activity to evaluate the tinnitus perception, event-related desynchronization (ERD) and synchronization (ERS) of EEG signals may be also very helpful, since they reflect important aspects of sensory, motor, and cognitive cortical processing. ERD and ERS have been successfully used to study the neural response in clinical cases such as pain evaluation (Peng \& Tang, 2016) and neuro-rehabilitation (Caimmi, et al., 2016). The enhancement of ERD/ERS quantification must be, however, considered as well. $\mathrm{Hu}$ and his workmates demonstrated that the conventional method proposed by Graimann and Pfurtscheller (Graimann \& Pfurtschelle, 2006) to quantify ERD/ERS introduces a positive bias, resulting in an overestimation of ERS and an underestimation of ERD. Authors proposed to combine single-trial baseline subtraction approach, in conjunction with partial least square regression, to achieve a correct detection and quantification of ERD/ERS (Hu, et al., 2014). 
In view of the above discussion, the analysis of spontaneous, evoked, and induced EEG activity using improved techniques could lead us to evaluate properly the effectiveness of acoustic therapies to treat tinnitus. In addition, the consideration of other EEG measures such as phase, neural generators, and cross-frequency-coupling might fulfil a broad and thorough assessment of acoustic therapies (Jia, et al., 2015).

\subsubsection{ANALYSIS TOOLS}

To undertake the EEG signal processing, a high level programming language is used: MATLAB. EEGLAB ${ }^{9}$ is an open-source MATLAB toolbox for processing continuous and event-related EEG signals.

\subsection{PROCEDURE}

\subsubsection{STUDY DESIGN}

Once patients agreed to participate and a consent form was signed, they were randomly assigned into one of four groups ${ }^{10}$ : RS, TRT, TEAE or binaural. They were instructed to use the assigned therapy for one hour, every day, and at any time of the day. The therapy was monitored for eight weeks as is depicted in Figure 7. During that span, four EEG recordings and three questionnaires (Section 2.6.1.2) were conducted. The first EEG recording was taken at the beginning of the study. The other three recordings were carried out one, five and eight weeks after therapy initialization.

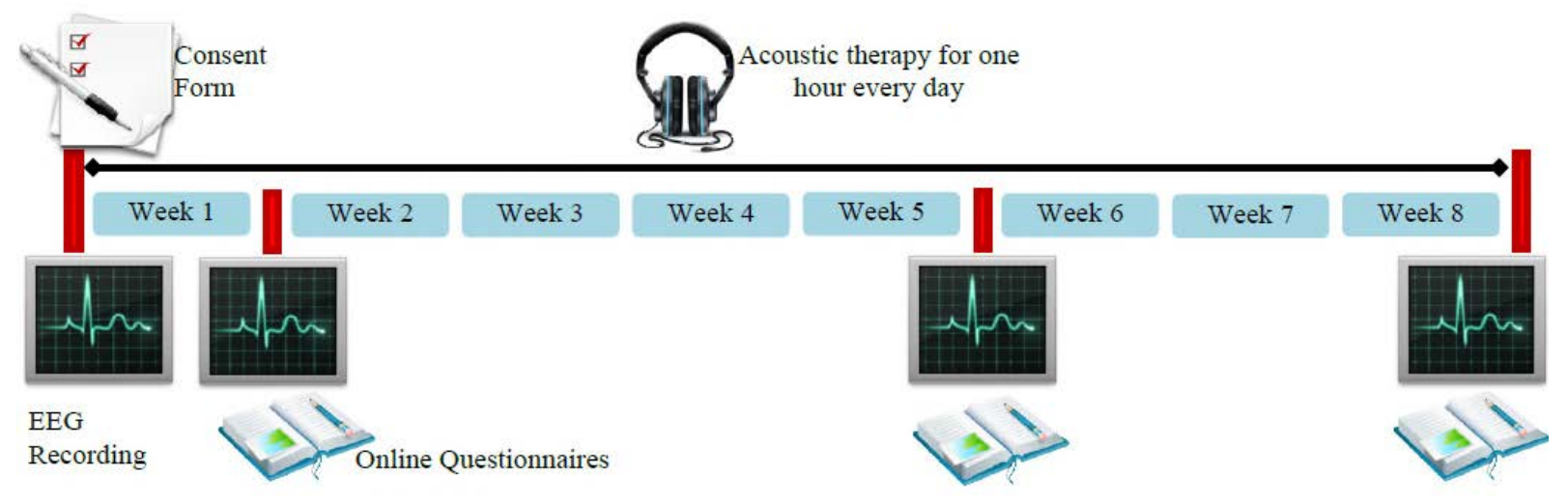

Figure 7: Study design of the protocol. Patients monitored for 8 weeks

The pulse was checked in the first and last sessions in order to monitor the changes and/or possible improvements.

EEG data was recorded in four different conditions: rest, acoustic therapy, passive mode and active mode. Each EEG session lasted around 60 minutes and was organized as shown in Table 1. As can been seen from the table, resting condition

\footnotetext{
${ }^{9}$ EEGLAB is available at https://sccn.ucsd.edu/eeglab/

${ }^{10}$ Generic acoustic therapies in use are available at https://doi.org/10.6084/m9.figshare.5558779.v1
} 
(Section 2.6.1.4) and acoustic therapy condition (Section 2.6.1.5) were recorded in session 1 and session 4, passive mode condition (Section 2.6.1.6) was taken in the four sessions, and active mode condition (Section 2.6.1.7) was taken from second session to ahead.

\begin{tabular}{|c|c|c|c|c|c|}
\hline & & \multicolumn{4}{|c|}{ Total duration per session (min) } \\
\hline & & Session 1 & Session 2 & Session 3 & Session 4 \\
\hline & total (min) & 45 & 53 & 53 & 65 \\
\hline \multirow{8}{*}{ 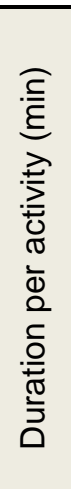 } & 15 & Consent form & & & \\
\hline & 3 & Check pulse & & & Check pulse \\
\hline & 15 & & \multicolumn{3}{|c|}{ Questionnaires } \\
\hline & 15 & \multicolumn{4}{|c|}{ Electrode mounting } \\
\hline & 6 & $\mathrm{EO} / \mathrm{EC}$ & & & EO/EC \\
\hline & 3 & Therapy & & & Therapy \\
\hline & 3 & \multicolumn{4}{|c|}{ Passive mode } \\
\hline & 20 & & Restaurant & Park & $\begin{array}{l}\text { Construction in } \\
\text { progress }\end{array}$ \\
\hline
\end{tabular}

Table 1: Organization of the experimental procedure

\subsubsection{Checking pulse}

Counting of arterial pulse per minute is equivalent to measuring the heart rate. It was evaluated the pulse at the wrist (radial artery) using a stethoscope during 30 seconds and calculating the Bpm afterwards. The patient was sit and relaxes in the course of this activity.

These measurements were done during two sessions:

- The first one, before the electrode mounting

- The fourth (and last one) before answering the questionnaires

\subsubsection{Questionnaires}

In most of the cases, the effectiveness of the acoustic therapies to treat patients suffering from tinnitus is subjective evaluated by means of a Visual Analog Scale (VAS) and/or ad hoc questionnaires (see Annex M for more details).

It has been applied two online questionnaires (see Annex N), filled in at three different spans: one, five and eight weeks after treatment initialization. These questionnaires are intended as a psychological and subjective measurement to assess the tinnitus itself and the emotional or behavioural irregularities related to it.

- The psychological part is based on the Hospital Anxiety and Depression Scale (HADS) developed by Zigmond and Snaith (Zigmond \& Snaith, 1983).

- The tinnitus assessment is an ad-hoc version of the THI created originally by Newman and colleagues (Newman, et al., 1996) 
- The original THI questionnaire has 25 questions.

- It used to monitor

- degree of tinnitus perception

- physical, emotional, and social response to tinnitus

- interfering effect of tinnitus on hearing ability

- It was adapted and simplified from the INR (named as CIA.EVO ${ }^{11}$ ) to an already validated and reliable lighter version of 10 items and is refers to as tinnitus evolution questionnaire.

As questionnaires are a subjective / psychological measurement that can reveal emotional and behavioural irregularities related to tinnitus, there were later associating with EEG outcomes.

\subsubsection{Electrode mounting}

As explained in 2.4.2 Equipment and software and illustrated in Figure 6b, seventeen of the nineteen electrodes were used according to the 10/20 international system. The $\mathrm{Cz}$ channel was used for referencing the other sixteen EEG channels, and left lobe ear worked as ground.

The electrode mounting process was tedious. A gel was injected into the central part of the electrode so that the electrode contacting the scalp could reduce the impedance of the electrode. The main criteria was to reduce the impedance below $10 \mathrm{~K} \Omega$. It depended on the size of the head and the length and type of hair of the patient.

\subsubsection{Resting condition}

Resting condition was recorded in session 1 and 4, what means at the beginning of the study when no acoustic therapy were applied yet and at the last session to understand if there was any change.

Patients were asked to sit down on a comfortable chair, to be relax, and to keep their eyes open (EO) for three minutes. Their vision was directed to a fixation point on the computer screen in use. After EO period, they were asked to keep their eyes close (EC) for the following three minutes. Although EO and EC conditions have been the most examined brain state in neuroimaging studies related to tinnitus perception, no agreement on which condition is most reasonable, or what duration is more convenient, has been reached yet. Either EO or EC, and duration variation from two (Balkenhol, et al., 2013) up to ten minutes (Pierzycki, et al., 2016) have been reported in previous studies.

In the present thesis, it has been proposed to record periods of three minutes, in line with prior research conducted to determine neurophysiological parameters that depend

${ }^{11}$ CIA: Cuestionario Inicial de Acúfenos ; CIA.EVO: Cuestionario Evolución de Acúfenos 
on mental states at rest such as level of attention ( $\mathrm{Li}, 2010)$, alpha peak frequency (Posthuma, et al., 2001), and level of motor imagery ability (Blankertz, et al., 2010). With respect to the selection between EO and EC, and even though both conditions are recorded at this stage, EC seems to be more reasonable to evaluate the tinnitus perception in the rest of the experimental stages (acoustic therapy, passive mode, and active mode). Adjamian (2014) reported that enhanced gamma activity, which had been previously related to tinnitus perception, most probably proceeded from involuntary eye movements and saccades, rather than abnormal neural activity due to tinnitus. On the other hand, Geller and his co-workers (Geller, et al., 2014) associated eye closure with diminution of high frequency brain oscillations. Taken together, these results suggest that if gamma ERS exists owing to tinnitus perception, such neural synchronization should be more detectable in EC, than in EO condition. In addition to this evidence, $\mathrm{Li}$ ( $\mathrm{Li}, 2010$ ) argued that alpha band rhythms can work as biomarker to differentiate brain states that require different attentional degrees such as EC and EO. As EO involves interaction with the environment (external attentional process), and tinnitus perception is an internal attentional process, EC might be a more effective condition to study the effect of acoustic therapies during the auditory retraining.

\subsubsection{Acoustic therapy}

This condition was recorded at the beginning and during the last session (fourth session), when the assigned acoustic therapy was already applied for two months.

Similar to previous condition, patients were asked to keep their EC for three minutes while they listen to their acoustic therapies. As was aforementioned, this condition (acoustic therapy) and the rest of them (passive and active modes) were only monitored in EC mode.

EEG mapping of tinnitus perception during acoustic therapy should reveal if there was any neural synchrony modification as a result of the treatment, in comparison with resting condition when this was not received yet. In this way, if any beneficial effects would have been produced during acoustic retraining, these should be reflected in this particular recording. An important point to remark is that the main goal of the thesis is to evaluate the therapy effect (independent variable), rather than tinnitus perception per se. Consequently, acoustic stimulation always depends on the acoustic therapy in use.

\subsubsection{Passive mode}

Passive mode is the only condition that is recorded in all the sessions: from session 1 to session 4. As the preceding condition, EEG recordings are only made with EC. In passive mode, auditory ERPs were examined, and for that purpose, stimulus with duration of $1 \mathrm{~s}$ and an inter-stimulus interval of $2.5 \mathrm{~s}$ were used. In total, 50 trials were taken, and hence each session lasted around two and a half minutes. 
Auditory ERPs have been extensively employed to study the tinnitus nature, and generally, $1 \mathrm{kHz}$ tones have been preferred because of convenience (optimum responses over the auditory cortex), rather than for relevance. In other investigations, tinnitus sound has been emulated, and subsequently, it has been used as auditory stimulus (Adjamian, 2014). However, it is important to keep in mind that tinnitus frequency response curve is subjectively obtained in humans (Roberts, et al., 2006), and it would depend on patients' perception to establish auditory stimulus, what in turn could produce subjective outcomes. The use of auditory stimulus based on the acoustic therapy at hand would standardize the evoke response, at least in each group. Acoustic therapies are moreover the subject of the study, and it seems plausible to provide the same auditory stimulation used to achieve acoustic retraining, as well as to assess such retraining.

\subsubsection{Active mode}

Active mode was recorded in sessions 2, 3 and 4, and EC was requested as well.

In each session, a usual acoustic environment is played, whilst five associated auditory stimuli are randomly played. Patients were instructed to identify the randomized stimuli by pressing a keyboard button. The three acoustic environments along with their related stimuli in each session were standardized to $1 \mathrm{~s}$ and repeated 50 times at a random rate. They were the following ${ }^{12}$ :

(1) restaurant - soda can being opened, door closing, glass breaking, microwave sound, and human sound (tasting food)

(2) park - camera clicking, book page turning, cards shuffling, human sound (laughing), and human sound (whistling)

(3) construction in progress - hit, bang, mobile dialling, police siren, and human sound (yelling).

Although tinnitus perception has been related to abnormal neural activity in the auditory system, tinnitus distress has been associated with co-activation of frontal, limbic, memory and automatic systems (Adjamian, 2014; Krause, 2003). Memory is of particular interest because it is a sensory representation of the world, which allows humans interacting with their environment. In essence, new input patterns are first decoded and storage in the long-term memory of the brain. When those patterns appear again, brain retrieves information from memory, and makes a prediction. In this way, the consumption of mental resources is reduced (Karakas, 1997). What is pursued in active mode registry is to investigate if patients suffering from chronic tinnitus have memory

\footnotetext{
${ }^{12}$ Acoustic environments and the corresponding stimuli to be identified in each situation can be listened at https://doi.org/10.6084/m9.figshare.5558752.v1
} 
atrophies, by evaluating their reaction time to recognize common sounds in typical environments. This EEG evaluation may be a way to assess indirectly tinnitus distress.

At present, no reliable EEG-based methods have been found. In fact, some authors (Pierzycki, et al., 2016) consider that EEG is not an effective way to study tinnitus effects. On this evidence, several EEG evaluations have been proposed in this thesis: from no specific auditory stimulation (resting condition), to auditory pattern recognition (active mode). This set-up will also lead us to propose an efficient EEG-based methodology to study tinnitus effects.

\subsubsection{POST-STUDY TREATMENT}

In the case that patients didn't report either habituation or suppression to tinnitus during the two months treatment, standard versions of acoustic therapies were provided to patients, if they decided to continue with the post-study treatment. Only three of the four therapies in use were applied in post-study treatment because the one originally assigned to the patient was discarded. Patients were instructed to use each therapy every day for 30 minutes and during two weeks. After this testing period, patients will report if they detected any positive change. If any, the therapy of interest will be adjusted to the patient audiometry. For the post-study treatment stage, no EEG monitoring will be undertaken since it was beyond the scope of the thesis.

\subsubsection{LIMITATIONS AND TROUBLESHOOTING}

A limitation of this proposal is the guarantee of acoustic therapy application as is indicated. This procedure cannot be verified as drug administration can be via blood test. In order to increase reliability, only liable patients were recruited according to a previous filtering process undertaken in the INR. Furthermore, patients were notified about the experimental procedure, and they needed to sign a compromise statement, agreeing to take part in the program.

Another limitation of the proposal is the number of EEG channels. As can be seen from Figure 6, only 16 of the 19 electrodes of the 10/20 system were used. However, although it was considered that the small number of available channels may restrict somehow the study, the estimation of EEG measures is still feasible and reliable. It is well-known that the spatial resolution of EEG is very poor and underdetermined, even if a large number of sensors are used. EEG signals are smeared as they pass through the surrounding tissue and the poorly conductive skull. Furthermore, the surface distribution of electrical currents is also somewhat distorted since the conductivity and the thickness of the skull is non-uniform (Adjamian, 2014). The determination of signal sources is, therefore, complex and inaccurate due to EEG nature, rather than the number of sensors in use. Up to now, data acquisition in previously conducted EEG-based studies has been made using from 19 (Vanneste, et al., 2013) to 128 (Meyer, et al., 2014) 
sensors. As can been seen, consensus has not been reached yet, and even the use of too many channels does not guarantee the analysis of all of them. For instance, Meyer and his colleagues (Meyer, et al., 2014) reduced the number of electrodes from 128 to 109 channels by omitting the outermost ring of electrodes, as they usually show high amounts of noise. Lastly, the study of certain EEG measures such as ERPs is commonly undertaken only over one or two recording sites, what is achievable by means of the EEG layout depicted in Figure 6. In conclusion, it was considered that recordings of 17 EEG channels could be exploited to obtain consistent results as has been exemplified in the Section 3 Results.

\subsection{ETHICS}

The protocol was approved by the Ethical Committee of the National School of Medicine of the Tecnologico de Monterrey. The ethical clearance (CONBIOETICA19CEI00820130520) was obtained on June $20^{\text {th }} 2016$ (see Annex O).

Apart of that, it was submitted on October $31^{\text {th }} 2017$ under Registration Number 14553550 to ISRCTN Registry: BioMed Central ${ }^{13}$ ).

As explained, the research project was undertaken in collaboration with the INR in Mexico City, where patients with chronic and refractory tinnitus interested in being treated with acoustic therapies were recruited for the study. When inviting the participants, the purpose of the research is clearly explained. After obtaining written informed consent from the patient, the experimental procedure takes place.

\footnotetext{
${ }^{13}$ https://doi.org/10.1186/ISRCTN14553550
} 


\section{RESULTS}

While All is in The All, it is equally true that The All is in All

Democritus (60 A.D.), Greek philosopher 


\subsection{INTRODUCTION}

For this thesis and due to the difficulties to get patients and the temporal constraints of the experimentation itself, it has been considered the results of the first part of the methodology explained in the Section 2. Materials and Methods, what means the passive measurements which will be analysed mainly through the ERPs (from the objective point of view) as explained. Those measurements are enough to cover and reach the objectives of the thesis. The active measurements from the patients will be treated in a future research.

Apart of that and during the following chapter, it will be explained the subjective data analysis collected from the questionnaires what will be the basis to correlate passive and active information from the objective analysis.

It will be discussed the results in the following Section 4. Discussion and Conclusions.

\subsection{SUBJECTIVE ANALYSIS}

As explained in the 2.6.1.2 Questionnaires, it was applied two online questionnaires (see $\underline{\text { Annex N}}$ ), filled in from the second session onwards.

In order to show the result of the questionnaires, It has been selected a total of 58 (from the 68) patients (which means 85\%). Those numbers are linked to the ones they finished all the 4 sessions and they had a minimum of patients per acoustic therapy applied: Relaxing Music (RS), TRT, EAE, Binaural $f_{o}$, Binaural $396 \mathrm{~Hz}$ and Binaural $432 \mathrm{~Hz}$. For example binaural with $432 \mathrm{~Hz}$ carrier frequency was ruled out because there were only 2 samplers with 4 sessions completed.

\begin{tabular}{|c|c|}
\hline Acoustic Therapy & Number people \\
\hline RS & 13 \\
\hline TRT & 17 \\
\hline EAE & 16 \\
\hline Binaural & 12 \\
\hline
\end{tabular}

Table 2: Number of Patients who completed the 4 sessions per Therapy

From those 12 Patients treated with Binaural Therapy, two out the three different carrier frequencies are represented (all of them with a $10 \mathrm{~Hz}$ difference as explained previously) 


\begin{tabular}{|c|c|}
\hline Carrier frequency & Number people \\
\hline $396 \mathrm{~Hz}$ & 8 \\
\hline$f_{o}$ (Tinnitus freq.) & 4 \\
\hline
\end{tabular}

Table 3: Number of Patients who completed the 4 sessions of Binaural Therapy

At the end of the last session, every Patient was asked about a self-assessment of the acoustic therapy they were following. The most of the patients respond about it. The first result is showing Binaural therapy with a much better improvement than any other therapy, follow by TRT.

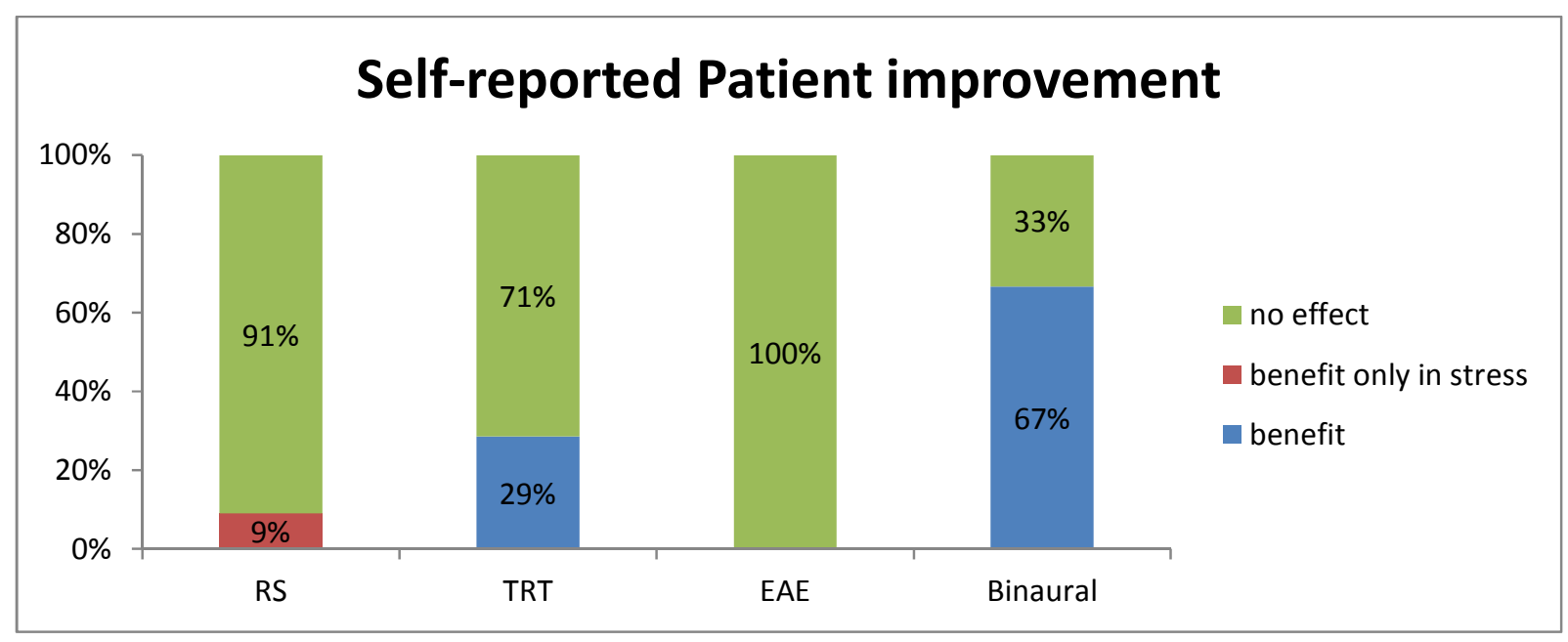

Figure 8: Self-reported patient improvement per Therapy

On the other hand and in order to see the evolution of the different patients about their specific therapies, it has been calculated the score of the answers of all the questions from the questionnaires. As explained before, CIA.EVO (ad-hoc solution from INR) score has been calculated based on THI (Aparecida de Azevedo, et al., 2007; Newman, et al., 1996) where 25 questions have been reduced to 10 to see the evolution of Tinnitus. Anxiety and Depression results have been identified according to the HADS tools and process (Annunziata, et al., 2011; Zigmond \& Snaith, 1983; Levenstein, et al., 1993) based on 14 questions (odd related to anxiety and even to depression). In the following tables are explained the scale associated with that total score determined from the answers to the questions. 


\begin{tabular}{|c|c|c|}
\hline $\begin{array}{c}\text { Total score } \\
\text { CIA.EVO }\end{array}$ & $\begin{array}{l}\text { THI } \\
\text { score }\end{array}$ & Scale \\
\hline $0-10$ & $0-16$ & 1: Mild (Only perceived in silent environments) \\
\hline $11-23$ & $18-36$ & $\begin{array}{l}\text { 2: Light (Easily masked out by environmental noise and } \\
\text { easily forgotten with daily activities) }\end{array}$ \\
\hline $24-36$ & $38-56$ & $\begin{array}{l}\text { 3: Moderate (Perceived even in the presence of background } \\
\text { noise, although it does not prevent daily activities) }\end{array}$ \\
\hline $37-49$ & $58-76$ & $\begin{array}{c}\text { 4: Severe (Perceived almost in any situation, impacts sleep } \\
\text { patterns and may interfere with daily activities) }\end{array}$ \\
\hline$>49$ & $78-100$ & $\begin{array}{l}\text { 5: Catastrophic (Always present, impairs sleep, impairs the } \\
\text { performance of any activity) }\end{array}$ \\
\hline
\end{tabular}

Table 4: Tinnitus perception scale based on CIA.EVO total score calculation

\begin{tabular}{|c|c|}
\hline Total score D / A & Scale \\
\hline $0-7$ & 1: Normal \\
\hline $8-10$ & 2: Borderline abnormal (borderline case) \\
\hline $11-21$ & 3: Abnormal (case) \\
\hline
\end{tabular}

Table 5: Depression and Anxiety scale based on HADS total score calculation

The Annex $\mathrm{P}$ consolidates the score of the answers associated to the different questionnaires by patient during all the sessions.

It can be seen in the following graphs and tables the difference between the scales calculated from the questionnaires of the last and second session in order to know the improvement per group of patients per therapy and focusing in the bundled questions: Tinnitus perception, Depression and Anxiety. Those results mean that the more negative is the number, the better the patient improvement is. For example, a result of "2 " means that it was an improvement of 2 points in the scale of the assessment from the $2^{\text {nd }}$ and last session's answers.

\subsubsection{TINNITUS PERCEPTION EVOLUTION}

As figure 9 revealed, there is no clear therapy with a better performance for improving the tinnitus perception.

- In the most of the patients there is not sense of getting better.

- TRT, Binaural at $396 \mathrm{~Hz}$ and EAE therapies have $29 \%, 25 \%$ and $19 \%$ respectively of the patients having a better subjective impression. 
- Binaural at tinnitus frequency $\left(f_{o}\right)$ has not effect.

- RS is the worst therapy of all. On the other hand Binaural at $396 \mathrm{~Hz}$ had a case of worsening too.
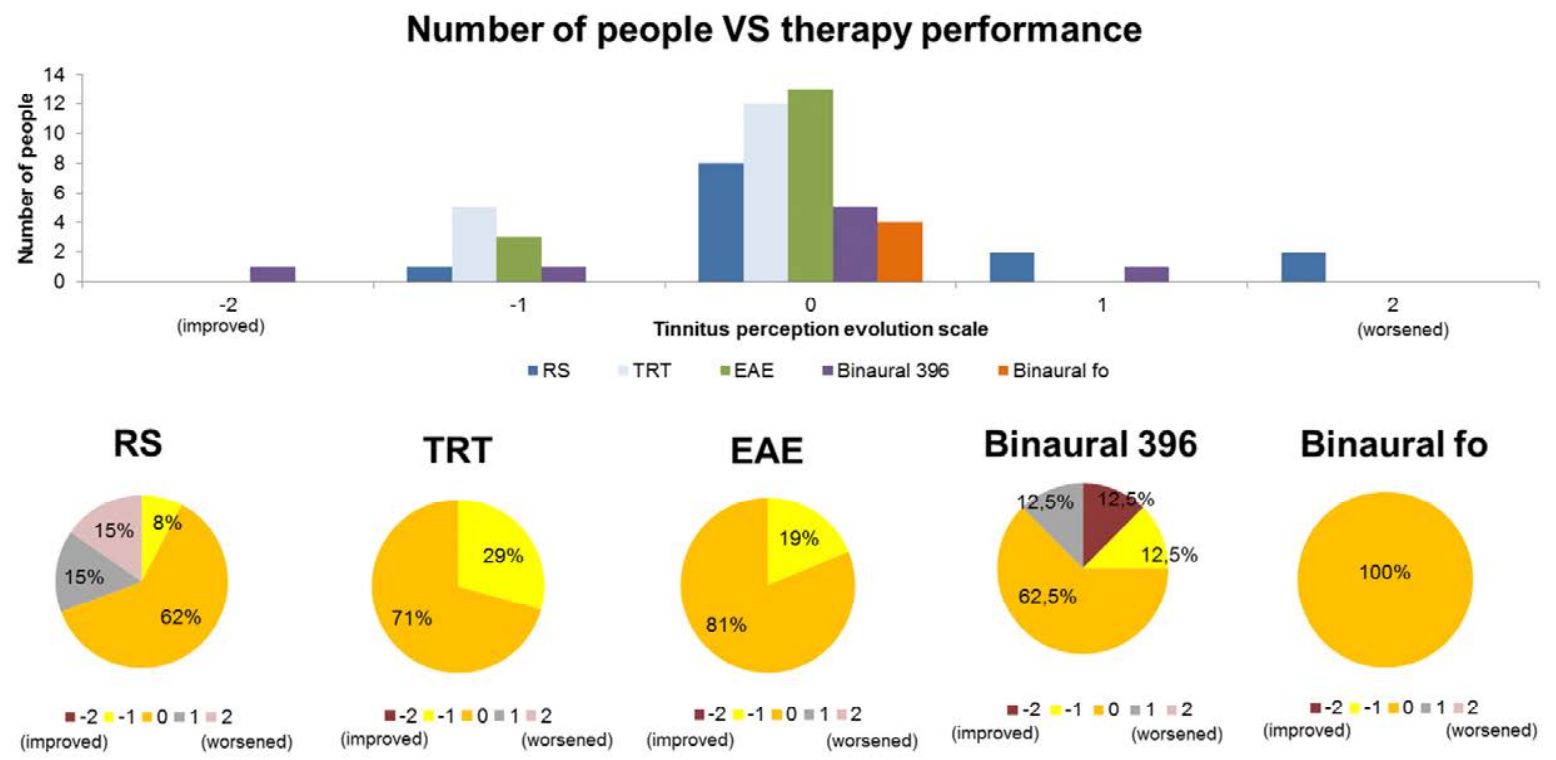

Figure 9: evolution of Tinnitus perception scale per Therapy

In the table below, it was calculated the average $(\bar{x})$ and standard deviation $(\sigma)$ from the different therapies to express their performance as Gaussian curves. Again, it is shown that from the 3 best signals explained before with almost the same insight achievement, TRT and EAE are more accurate (smaller $\sigma$ ) than Binaural $396 \mathrm{~Hz}$.

\begin{tabular}{|c|c|c|}
\hline Therapy & $(\bar{x})$ & $\sigma$ \\
\hline RS & 0,38 & 0,87 \\
\hline TRT & $\mathbf{- 0 , 2 9}$ & $\mathbf{0 , 4 7}$ \\
\hline EAE & $\mathbf{- 0 , 1 9}$ & $\mathbf{0 , 4 0}$ \\
\hline Binaural 396 Hz & $\mathbf{- 0 , 2 5}$ & $\mathbf{0 , 8 9}$ \\
\hline Binaural $\mathrm{f}_{\circ}$ & 0 & 0 \\
\hline
\end{tabular}

Table 6: Average and standard deviation calculation from the evolution of Tinnitus perception scale per Therapy.

Deepening through the different initial CIA.EVO, it can be observed that all the possible cases are not contemplated with same samplers (number of patients) per therapy and Tinnitus perception scale so it should not be a perfect match and comparison with all of them. Annex Q details the data from the different initial CIA.EVO and their conclusions. 
Finally and after all this analysis, if one therapy should be chosen to improve the subjective Tinnitus perception from all of the 5 implemented ones, it should be TRT.

\subsubsection{ANXIETY EVOLUTION}

As it can be seen in the figure 10, there is not a big different between any of the therapies applied to reduce Anxiety.

- All of the signals but Binaural $f_{o}$ have some patients where they were improving but nothing relevant in comparison with the overall result.

- Nevertheless and analyzing the Annex R table which describe the average $(\bar{x})$ and standard deviation $(\sigma)$ of the different therapies linking them with a Gaussian curves, EAE is the most pursuance for that with a better veracity and preciseness.
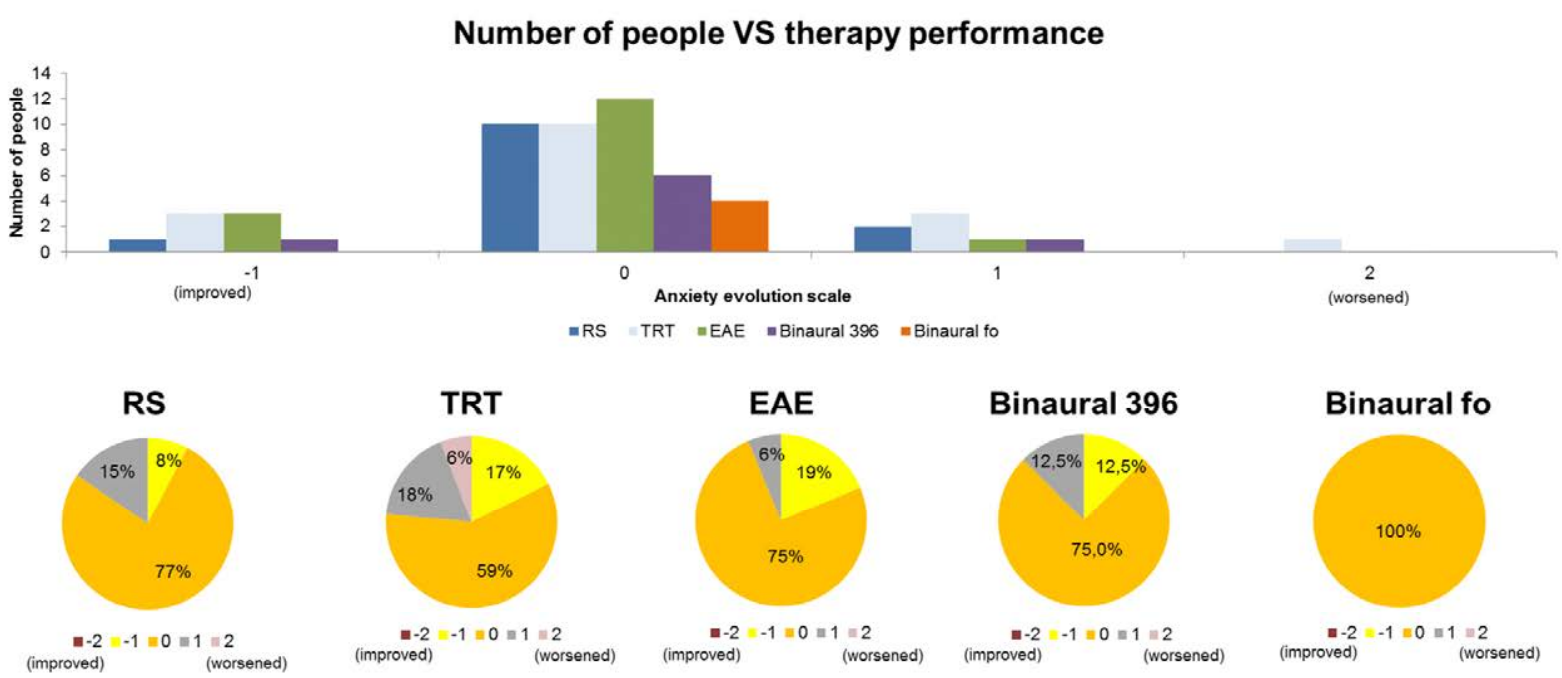

Figure 10: evolution of Anxiety scale per Therapy

The resulting Annex $R$ second table considers the various initial Anxiety (HADS-A) scales and adjust the previous calculation taking into account that first phase.

- It can be understood that for the scale 1, there is not roof for improvement and the most of the patients (72\%) they were already considered as "normal" status. Therefore, no significant information to add value.

- HADS-A=2 improvement has a better performance in TRT and EAE with quite similar number

- Binaural $396 \mathrm{~Hz}$ has the best and unique progress in the group of patients with an abnormal case (HADS-A=3)

Certainly and due to the evidences already explained, it should be chosen Binaural 396 $\mathrm{Hz}$ for Anxiety recovery with higher HADS-A (worst case scenario) and EAE or TRT for lower ones. 


\subsubsection{DEPRESSION EVOLUTION}

It may be notice from figure 11 that scaling down Depression has a similar behavior than Anxiety (figure 10)

- Binaural $f_{o}$ keeps maintaining the "no effect" achievement.

- EAE and more significantly TRT carried some good execution but really bad ones too, what brings a huge range of possibilities.

- RS had a patient with a minor improvement

- Binaural $396 \mathrm{~Hz}$ is the first-rate with $37 \%$ amelioration.
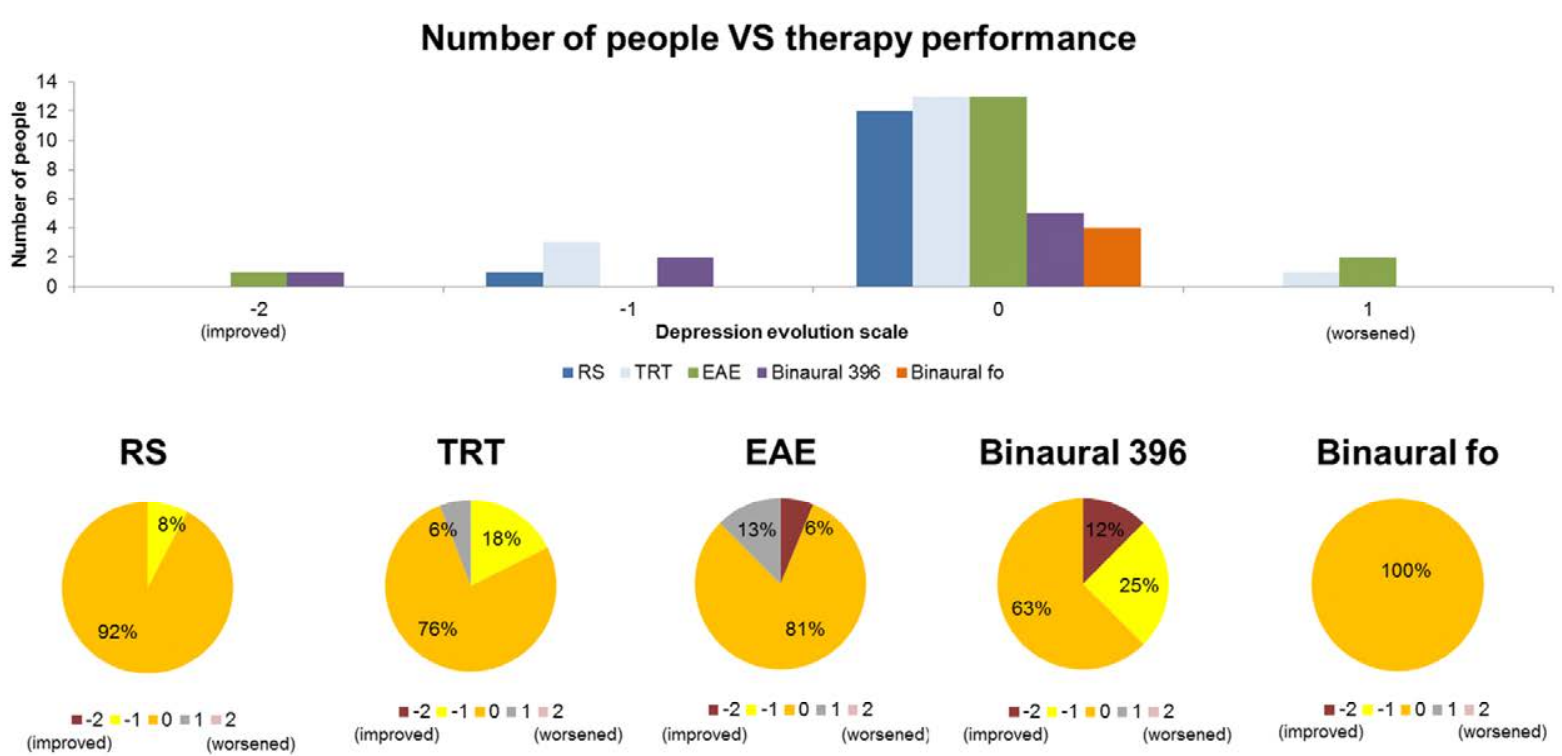

Figure 11: evolution of Depression scale per Therapy

The subsequent table in Annex S characterizes the average $(\bar{x})$ and standard deviation $(\sigma)$ of the different therapies applied. Binaural $396 \mathrm{~Hz}$ is the better position

Digging out a level below and evaluating the particular initial Depression (HADS-D) scales, Annex S second table main conclusions are

- The vast majority of the patients (83\%) were already in phase 1 (normal). Hence, it is tough to bring any evolution.

- Relying on the few remaining samplers for HADS-D $=2$ and 3 , it can be identified that even if TRT and EAE have some positive figures, Binaural $396 \mathrm{~Hz}$ has a more systematic and valuable outcome

Lastly and by cause of the earlier justifications, the preferred therapy for Depression improvement is Binaural $396 \mathrm{~Hz}$. 


\subsection{OBJECTIVE ANALYSIS}

\subsubsection{PULSE RATE}

There are some studies and research explaining that being relax or meditating have positive effect on sympathovagal balance. Some of them have been demonstrated through data obtained from fMRI (Telles, et al., 2014), EEG (Telles, et al., 2018) and heart rate variability (HRV), blood pressure $(\mathrm{BP})$ and heart rate $(\mathrm{HR})$ with similar outcomes (Aryaa, et al., 2018).

As commented in 2.6.1.1 Checking pulse, it has been measured and evaluated the difference from last and first session (in Bpm).

In most of the cases, the sympathetic nervous activity stays at the same level. Binaural therapies, TRT and EAE improve (reducing the Bpm) in some of the patients as represented in the Table 7, which has a direct link with the Anxiety Evolution already described. On the other hand, TRT and RS are the most probable therapies of getting worse (increasing the Bpm). Therefore, for this particular assessment, scope and data, it could be said that Binaural (specifically $396 \mathrm{~Hz}$ ) is the best of all for this purpose.

\begin{tabular}{|l|c|c|c|}
\hline Therapy / Pulse evolution & $\begin{array}{c}\text { Reduce } \\
\text { (improve) }\end{array}$ & $\begin{array}{c}\text { Stay } \\
\text { same }\end{array}$ & $\begin{array}{c}\text { Increase } \\
\text { (get worse) }\end{array}$ \\
\hline RS & $8 \%$ & $77 \%$ & $15 \%$ \\
\hline TRT & $14 \%$ & $57 \%$ & $29 \%$ \\
\hline EAE & $13 \%$ & $80 \%$ & $7 \%$ \\
\hline Binaural 396 & $33 \%$ & $67 \%$ & $0 \%$ \\
\hline Binaural fo & $25 \%$ & $75 \%$ & $0 \%$ \\
\hline
\end{tabular}

Table 7: Percentage of people versus Pulse evolution per therapy

Elseways, the fact of understanding the tests and process to be faced by the patients it may be another reason to reduce the Bpm along the sessions, where in the first one they could be nervous with a higher Bpm.

\subsubsection{EEG DATA PROCESING AND MEASUREMENTS}

Figure 12 explains the steps followed to process the EEG data from the sixteen channels analyzed. Every channel has recorded a signal approximately of 3 minutes. According to Castellanos \& Makarov (2006) to keep the output of the signal stable and controlled, the length of the segments shouldn't be longer than 15 seconds, and it is recommended to fix them to 10 seconds for ICA decomposition.

For time domain analysis, the mean of the segments is calculated. From that signal, the common statistical features are calculated. Those parameters are described in Annex T. Generally, in order to 
- Understanding severe irregularities: Mean (MeanAbs, Median, MMAV1, MMAV2, STD, VAR), Peak (Max, Min) and through Energy calculation methods such as Root, RMS, IEEEG, SSI or WL.

- Interpreting early deficiencies: Crest, Skewness, Kurtosis, Clearance, Shape, and Impulse.

Patient $X$ (Acoustic Therapy $Y$ ) per session 1-4

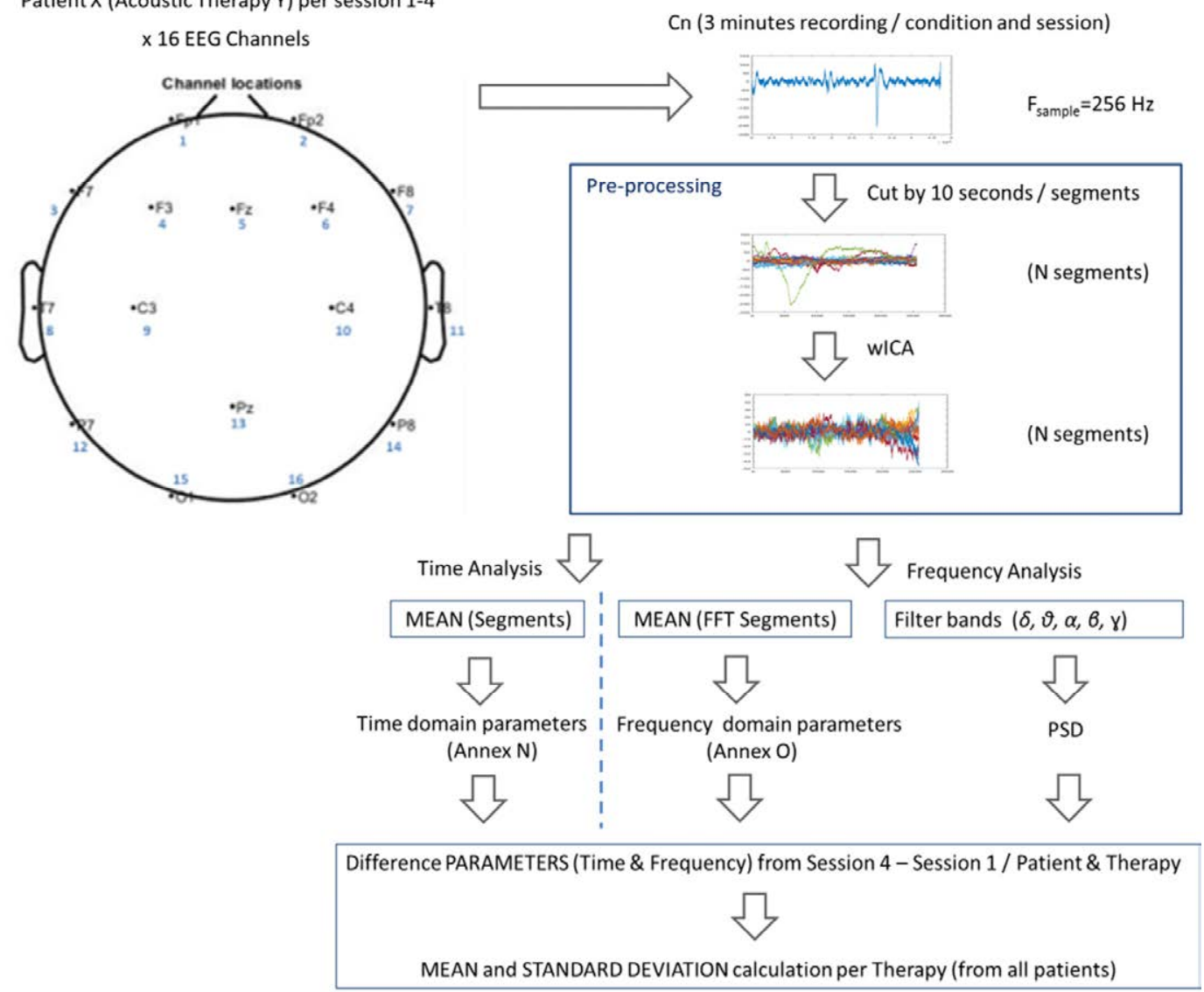

Figure 12: Mean and standard deviation process calculation per therapy from the difference of the time and frequency domain parameters of the patients

For frequency domain analysis, the Fast Fourier Transform (FFT) is calculated for every segment. The mean of those ones are determined afterwards. The common statistical features described in Annex $\mathrm{U}$ are computed from the final signal: $\mathrm{P} 1\left(\mathrm{X}_{\mathrm{mf}}\right), \mathrm{P} 2, \mathrm{P} 3, \mathrm{P} 4$, P5 $\left(M N F=X_{f c}\right), P 6, P 7\left(X_{r m s t}\right), P 8, P 9, P 10, P 11, P 12, P 13, P 14, M D F$ and Power Spectrum deformation.

On the other hand, a separation of bands $(\delta, \theta, \alpha, \beta, \gamma)$ is applied in order to understand the behavior ${ }^{14}$. The Power Spectral Density (PSD) ${ }^{15}$ is calculated for the total of the signal and every of those bands.

\footnotetext{
${ }^{14}$ More info in Annex F: Brain Oscillations Quantified in Tinnitus 
Finally, the mean and standard deviation are assessed per therapy from the differences between Session 4 and Session 1 per group of patients with same acoustic signal, and from the time and frequency domain parameters. For PSD, the result is the sum of the square of the differences between the samplers.

\subsubsection{Pre-processing Data}

Cerebral electrical activity measured by EEG scalp sensors is highly susceptible to contamination by both noncortical biologic artifacts (eg. Eye movements and blinks, muscle activity, cardiac pulse) and environmental noise (e.g. line noise, radio and electrical interference) A significant challenge for EEG research is the isolation and removal of artifacts and noise that contaminate the cortical signal of interest (Fiitzgibbon, et al., 2007).

It has been used wavelet enhanced ICA method (wICA) that applies a wavelet thresholding not to the observed raw EEG but to the demixed independent components as an intermediate step, allowing recovering the neural activity present in "artificial" components (Castellanos \& Makarov, 2006). The thresholding allows conservation of the time-frequency structure of artifacts and recovering of the cerebral activity "leaked" into the components. See the details of wICA algorithm in the Annex V.

\subsubsection{Resting condition (Eyes Open) Data}

The following Table 8 shows the average $(\mu)$ and standard Deviation $(\sigma)$ of a selection of time and frequency parameters calculated from the difference between last session and first session (relativized by session one, to understand and compare the evolution) of the data of all the patients of every acoustic therapy applied, during the resting condition (Eyes Open) as it was described in chapter 2.6.1.4 Resting condition.

Moreover, as explained before from previous researches (1.2 State of the art), Table 8 summarized the frontal and temporal EEG channels where tinnitus patients shown abnormalities comparing them with non-tinnitus subjects evaluated $(F p 1=1, F p 2=2$, $E 7=3, F 8=7)^{16}$.

- The frontal part (channels 1 and 2) is linked with the emotions and interpretation, where the stress may generate a defect.

- Temporal lobe (channels 3 and 7 ) is associated to decoding where a lack of perception may be generated.

\footnotetext{
${ }^{15}$ To calculate PSD for EEG data is recommended the use of the Welch's power spectral density (Ala, et al., 2018; Miskovic, et al., 2018; Ward \& Obeid, 2019; Pullon, et al., 2019; Rahman, et al., 2019)

${ }^{16}$ EEG channels location can be found in figure 21 and 10.b (chapter 2.4.2 Equipment and software)
} 


\begin{tabular}{|c|c|c|c|c|c|c|c|c|c|c|c|c|c|c|c|}
\hline & & \multicolumn{4}{|c|}{ Time domain parameters selection } & \multicolumn{10}{|c|}{ Frequency domain parameters selection } \\
\hline Binaur & & Mean & Peak & RMS & KURTOSIS & PSDtotal & PSD- $\delta$ & PSD- $\theta$ & PSD- $\alpha$ & PSD- $\beta$ & PSD- $y$ & $\mathrm{P} 1(\mathrm{Xmf})$ & P4 & P5(Xfc) & P13 \\
\hline \multirow{2}{*}{1} & $\mu$ & 9.69 & 0.16 & 0.45 & 0.24 & 3.76 & 45.78 & 1.67 & 0.52 & 0.34 & 0.35 & 0.03 & 50.63 & $-\quad 0.16$ & 0.04 \\
\hline & $\sigma$ & 13.68 & 0.68 & 0.50 & 0.02 & 5.02 & 64.40 & 2.07 & 0.24 & 0.23 & 0.21 & 0.11 & 71.41 & 0.17 & 0.11 \\
\hline \multirow{2}{*}{2} & $\mu$ & 23.09 & 0.52 & 0.75 & 0.14 & 8.96 & 57.60 & 4.10 & 1.06 & 0.46 & 0.58 & 0.11 & 90.24 & 0.41 & 0.09 \\
\hline & $\sigma$ & 13.82 & 1.33 & 1.62 & 0.03 & 2.14 & 51.03 & 1.87 & 0.05 & 0.30 & 0.28 & 0.17 & 65.00 & 0.08 & 0.12 \\
\hline \multirow{2}{*}{3} & $\mu$ & 3.35 & 0.23 & 0.33 & 0.25 & 5.21 & 28.86 & 1.16 & 0.43 & 0.49 & 0.61 & 0.11 & 5.18 & 0.22 & 0.09 \\
\hline & $\sigma$ & 7.83 & 0.66 & 0.64 & 0.02 & 6.49 & 38.69 & 0.35 & 0.18 & 0.38 & 0.21 & 0.12 & 6.07 & 0.30 & 0.07 \\
\hline \multirow{2}{*}{7} & $\mu$ & 13.59 & 0.16 & 0.05 & 0.06 & 19.79 & 124.75 & 4.76 & 1.09 & 0.46 & 0.59 & 0.32 & 89.12 & 0.47 & 0.23 \\
\hline & $\sigma$ & 20.49 & 0.99 & 0.77 & 0.22 & 2.41 & 106.93 & 5.09 & 0.57 & 0.09 & 0.07 & $\begin{array}{l}0.04 \\
\end{array}$ & 95.44 & 0.02 & 0.05 \\
\hline EAE & & Mean & Peak & RMS & KURTOSIS & PSDtotal & PSD- $\delta$ & PSD- $\theta$ & PSD- $\alpha$ & PSD- $\beta$ & PSD- $\gamma$ & P1(Xmf) & P4 & $P 5(X f c)$ & P13 \\
\hline \multirow{2}{*}{1} & $\mu$ & 12.41 & $\begin{array}{ll}-\quad 0.14 \\
\end{array}$ & $\begin{array}{ll}- & 0.14 \\
\end{array}$ & \begin{tabular}{|l|}
0.24 \\
\end{tabular} & 1.23 & 3.63 & 0.41 & 0.28 & \begin{tabular}{|l|}
0.31 \\
\end{tabular} & 0.38 & $-\quad 0.24$ & \begin{tabular}{|r|}
20.01 \\
\end{tabular} & 0.55 & $\begin{array}{ll}-\quad 0.19 \\
\end{array}$ \\
\hline & $\sigma$ & 22.65 & 0.60 & 0.62 & 0.53 & 0.69 & 3.54 & 0.32 & 0.22 & 0.14 & 0.16 & 0.39 & 28.12 & 1.38 & 0.32 \\
\hline \multirow{2}{*}{2} & $\mu$ & 6.18 & 0.13 & 0.13 & 0.14 & 1.19 & 3.24 & 0.60 & 0.40 & 0.39 & 0.46 & 0.34 & 2.67 & 0.43 & 0.28 \\
\hline & $\sigma$ & 11.96 & 0.61 & 1.09 & 0.32 & 0.94 & 4.93 & 0.28 & 0.25 & 0.15 & 0.08 & 0.38 & 6.06 & 0.96 & 0.32 \\
\hline \multirow{2}{*}{3} & $\mu$ & 23.05 & 0.23 & 0.61 & 0.06 & 1.41 & 4.49 & 0.49 & 0.25 & 0.64 & 0.82 & 0.24 & 16.53 & 0.06 & 0.16 \\
\hline & $\sigma$ & 36.47 & 0.75 & 1.49 & 0.12 & 1.48 & 7.82 & 0.22 & 0.06 & 0.50 & 0.69 & 0.36 & 32.21 & 0.22 & 0.26 \\
\hline \multirow{2}{*}{7} & $\mu$ & 0.11 & 0.04 & 0.07 & 0.10 & 0.70 & 0.83 & 0.42 & 0.32 & 0.20 & 0.18 & 0.17 & 1.83 & 0.27 & 0.14 \\
\hline & $\sigma$ & 2.56 & 0.79 & 0.81 & 0.32 & 0.26 & 0.12 & 0.29 & 0.19 & 0.06 & 0.02 & 0.27 & 4.90 & 0.45 & 0.21 \\
\hline TRT & & Mean & Peak & RMS & KURTOSIS & PSDtotal & PSD- $\delta$ & PSD- $\theta$ & PSD- $\alpha$ & PSD- $\beta$ & PSD- $y$ & P1(Xmf) & P4 & $P 5(X f c)$ & P13 \\
\hline \multirow{2}{*}{1} & $\mu$ & \begin{tabular}{|r}
.66 \\
\end{tabular} & $-\quad 0.23$ & $-\quad 0.13$ & \begin{tabular}{|l|}
0.02 \\
\end{tabular} & 1.02 & 2.43 & \begin{tabular}{|l|}
0.39 \\
\end{tabular} & 0.30 & \begin{tabular}{|l|}
0.36 \\
\end{tabular} & \begin{tabular}{|l|}
0.48 \\
\end{tabular} & $-\quad 0.15$ & 5.98 & \begin{tabular}{|l|}
0.07 \\
\end{tabular} & $-\quad 0.12$ \\
\hline & $\sigma$ & 27.32 & 0.39 & 0.54 & 0.19 & 0.66 & 2.57 & 0.26 & 0.25 & 0.22 & 0.29 & 0.35 & 7.75 & 0.42 & 0.27 \\
\hline \multirow{2}{*}{2} & $\mu$ & 8.32 & 0.25 & 0.19 & 0.15 & 0.59 & 1.12 & 0.45 & 0.32 & 0.37 & 0.59 & 0.15 & 0.08 & 0.26 & 0.12 \\
\hline & $\sigma$ & 20.88 & 0.63 & 0.51 & 0.27 & 0.21 & 1.09 & 0.27 & 0.27 & 0.11 & 0.34 & 0.31 & 1.19 & 0.71 & 0.23 \\
\hline \multirow{2}{*}{3} & $\mu$ & 0.53 & 0.26 & 0.44 & 0.06 & 7.14 & 26.56 & 2.71 & 1.03 & 0.55 & 0.38 & 0.05 & 4.47 & 0.06 & 0.01 \\
\hline & $\sigma$ & 8.95 & 1.09 & 1.29 & 0.15 & 14.34 & 56.81 & 5.86 & 1.95 & 0.71 & 0.19 & 0.57 & 7.52 & 0.37 & 0.37 \\
\hline \multirow{2}{*}{7} & $\mu$ & 0.78 & 0.22 & 0.51 & 0.02 & 1.22 & 6.12 & 0.68 & 0.39 & 0.27 & 0.30 & 0.04 & 20.13 & 0.03 & 0.04 \\
\hline & $\sigma$ & 3.02 & 0.73 & 1.25 & 0.32 & 0.76 & 6.17 & 0.32 & 0.22 & 0.16 & 0.17 & 0.21 & 38.79 & 0.25 & 0.16 \\
\hline \multicolumn{2}{|l|}{ RS } & Mean & Peak & RMS & KURTOSIS & PSDtotal & PSD- $\delta$ & PSD $-\theta$ & PSD- $\alpha$ & PSD- $\beta$ & PSD- $\gamma$ & $\mathrm{P} 1(\mathrm{Xmf})$ & P4 & P5(Xfc) & P13 \\
\hline 1 & $\mu$ & $-\quad 0.87$ & $\begin{array}{l}0.27 \\
\end{array}$ & 0.28 & $\begin{array}{l}0.02 \\
\end{array}$ & 1.48 & 0.79 & 0.39 & 2.21 & 1.99 & 2.55 & \begin{tabular}{|l|}
0.57 \\
\end{tabular} & 0.99 & 0.16 & 0.40 \\
\hline 2 & $\mu$ & 1.12 & 0.40 & 0.32 & 0.05 & 1.12 & 0.74 & 0.42 & 1.73 & 1.50 & 1.51 & 0.43 & 0.97 & 0.06 & 0.30 \\
\hline 3 & $\mu$ & 0.99 & 0.33 & 0.29 & 0.05 & 0.67 & 0.91 & 0.48 & 0.61 & 0.51 & 0.48 & 0.32 & 0.93 & 0.06 & 0.25 \\
\hline 7 & $\mu$ & 1.74 & 0.20 & 0.31 & 0.01 & 0.67 & 1.62 & 0.21 & 0.54 & 0.33 & 0.74 & 0.29 & 4.77 & 0.20 & 0.22 \\
\hline
\end{tabular}

Table 8: Difference between session 4 and session 1: time and frequency parameters selection for resting (EO) analysis 
The data for EO of all channels measured and time / frequency domain parameters calculated (from the difference between sessions 4 and 1) are described in the Annex $\underline{\text { W. }}$

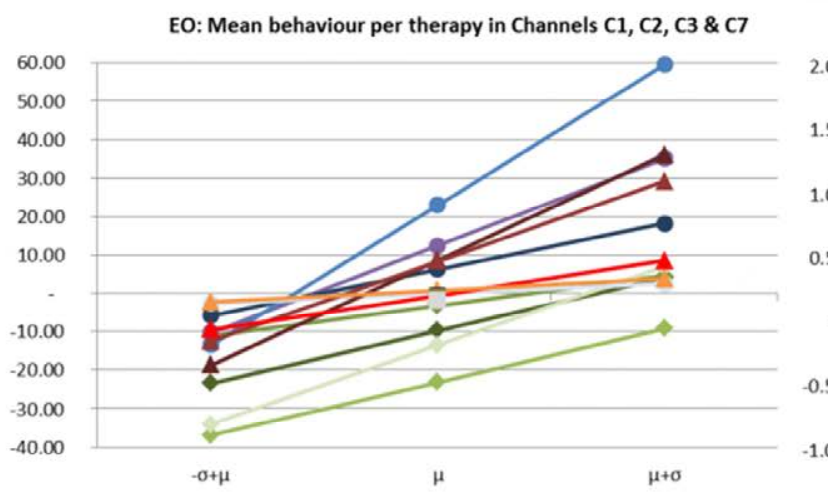

(a)

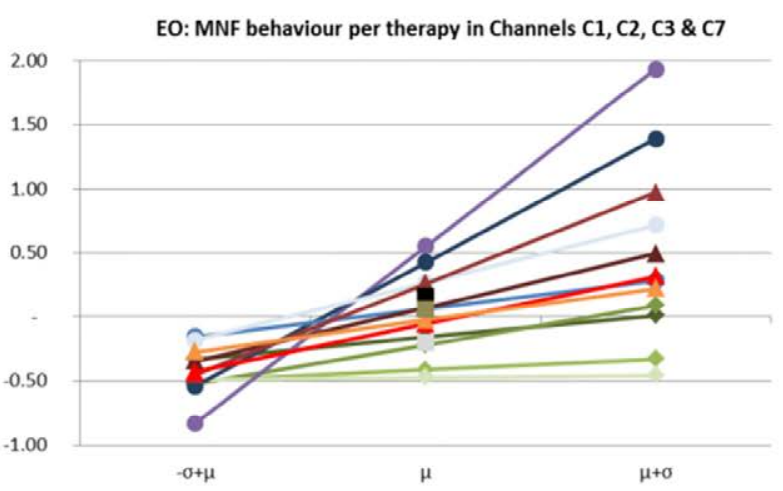

(b)

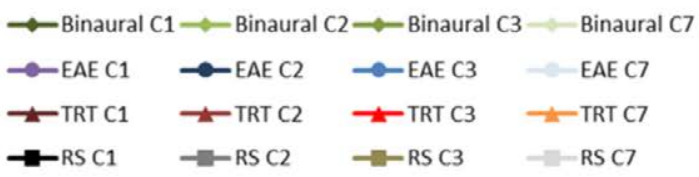

Figure 13: EO analysis: Frontal and temporal lobe behavior per therapy shown by the difference between session 4 and session 1 of the Mean (a) and MNF (b) representation

In terms of reduction of stress (frontal EEG channels location) using the acoustics therapies, it can be seen in Table 8 that Binaural $f_{0}$ has a slightly better performance (Peak and RMS) followed by TRT and EAE but when it is analyzed mainly the figure 13 (Mean and MNF), Binaural $f_{0}$ has much more advantage than any of the other therapies, followed by far by RS and much more further by TRT and EAE. What means in numbers: Binaural $f_{0}$ almost 10 times improvement, RS nearly no change, TRT bordering on 10 times worse, EAE more than 10 times worse.

The oscillation, peakness and symmetry of the signals don't reveal too much info to be compared because they are all quite similar.

Concerning channels 3 and 7 (temporal lobe), Binaural $f_{0}$ therapy seems to help to develop a better perception, followed by RS, TRT and EAE. Again, mainly represented by the mean: Binaural $f_{0} 3$ and 13 times improvement (respectively on left and right side) in contrast with the remaining therapies which are practically no changing with the exception of EAE left side with 23 times worse decoding capacity.

Regarding the frequency domain, the total spectral energy double in all the therapies but in Binaural $f_{0}$ where multiple by 4 times. Going deeper into the analysis, RS is moderately bigger for the bands $\alpha, \beta$ and $\gamma$, and Binaural $f_{0}$ for $\theta$. The energy distributed within the Band $\delta$ is much more extensive in the Binaural $f_{0}$ therapy than the others: 
- Frontal: Binaural $\mathrm{f}_{0}(\approx 50)$ followed by EAE / TRT $(\approx 3)$ and RS $(\approx 1)$

- Temporal lobe: Binaural $\mathrm{f}_{0}$ (29 and 124 on left and right side respectively) followed by TRT (26 and 6$)$, EAE (4 and 1$)$ and $R S(\approx 1)$.

\subsubsection{Resting condition (Eyes Closed) Data}

Following same rational explained previously and as a second part of the resting condition (Eyes Closed) clarified in 2.6.1.4, Annex X presents a selection of time and frequency parameters for the frontal and temporal lobe channels.

Regarding time domain analysis, the Peak, RMS and Kurtosis double the Binaural $\mathrm{f}_{0}$ behavior compared against the other acoustic therapies with similar performance. In connection with the Mean, Binaural $f_{0}$ is outlying the amelioration on both frontal and temporal lobe, followed by a long way by TRT and EAE. What it is defined by the channels 1, 2, 3 and 7 on TRT (7, 1, 3 and 5 times better), EAE (2, 0, 11 better but 15 times worse on channel 7 , the right side) and an improvement of Binaural $f_{0}$ up to more than 100 times for frontal and more than 150 for temporal left (with the exception of the right what is getting 40 times worse). An issue for Binaural $f_{0}$ is the presence of a big standard deviation due to the absence of data for these measurements.

In terms of frequency domain, the total spectral energy goes up to doubling for all therapies (in order TRT, EAE and RS) but in Binaural $f_{0}$ multiple by 5 times (channel 1 ) and 10 (remain channels: 2, 3 and 7). Understanding now the bands, it can be seen that similar energy distribution is in $\theta, \gamma$ and $\beta$, Binaural $f_{0}$ is vaguely bigger in $\alpha$ followed by RS. Band $\delta$ is more extensive in the Binaural $f_{0}$ therapy than the others:

- Frontal: Binaural $\mathrm{f}_{0}(\approx 20$ and 30 , channel 1 and 2 respectively) followed by TRT $(\approx 4$ and 2$), \mathrm{EAE}(\approx 3$ and 1$)$ and $\mathrm{RS}(0.5)$

- Temporal lobe: Binaural $\mathrm{f}_{0}$ (46 and 20 on left and right side respectively) followed by TRT ( $\approx 4$ and 5$), \operatorname{EAE}(\approx 1$ and 3$)$, and RS (0.5).

The data for EC of all channels measured and time / frequency domain parameters calculated (from the difference between sessions 4 and 1) are described in the Annex $Y$.

\subsubsection{Acoustic Therapy condition Data}

As it was explained in 2.6.1.5 Acoustic therapy, EEG patients functioning were recorded during 3 minutes listening their own assigned acoustic therapy at the beginning of the treatment and during the last session (fourth session, applied for two months).

Annex Z illustrates a representative extract of time and frequency parameters for the frontal and temporal lobe channels. In some cases there is a clear separation among left and right side performance for both frontal and temporal lobe: 
- Left frontal for Peak and RMS effectiveness: 5 and 6 times more for EAE followed by TRT $(\approx 1.5)$ and Binaural $396 \mathrm{~Hz}(\approx 0.5)$

- Left frontal for Mean: 3 times improved for Binaural $396 \mathrm{~Hz}$ followed by far getting worse by EAE (12) and TRT (225).

- Right frontal for Mean: 31 times improved for TRT followed by far by EAE (2) and getting worse by Binaural $396 \mathrm{~Hz}(6)$.

- Right temporal lobe for Peak and RMS behavior: $\approx 2$ times more for Binaural $396 \mathrm{~Hz}$ followed by TRT $(\approx 0.2)$ and EAE with an improvement of $\approx 0.25$ times.

- Left temporal lobe for Mean: 15 times improved for EAE followed by far getting worse by Binaural $396 \mathrm{~Hz}(1.5)$ and by TRT (3).

- Right temporal lobe for Mean: 48 times getting worse by Binaural $396 \mathrm{~Hz}$ followed by far by TRT (0.5) and getting better by EAE (0.7).

Concerning frequency domain, Binaural $396 \mathrm{~Hz}$ has a more focus $(<\sigma)$ behavior. Spectral activity in $\gamma$ and $\beta$ are similar but again, in some cases left/right and frontal/temporal are different:

- For the frontal activity

- total spectral energy EAE has a bigger impact ( 40 \& 2 times for left and right respectively), followed by TRT (16 \& 10) and Binaural $396 \mathrm{~Hz}(2.5 \& 1)$

- For $\delta$ band distributed energy TRT has a bigger impact (78 \& 7 times for left and right respectively), followed by EAE (45 \& 2) and Binaural $396 \mathrm{~Hz}(7 \& 2)$

- For $\theta$ band distributed energy EAE has a bigger impact $(6 \& 1$ times for left and right respectively), followed by TRT $(4 \& 3)$ and Binaural $396 \mathrm{~Hz}(1 \& 0.5)$

- For $\alpha$ band distributed energy TRT has a bigger impact $(3 \& 2.5$ times for left and right respectively), followed by EAE $(1.5 \& 0.5)$ and Binaural $396 \mathrm{~Hz}(0.2)$

- For the temporal lobe activity

- total spectral energy TRT has a bigger impact (37 \& 3 times for left and right respectively), followed by EAE $(1 \& 0.5)$ and Binaural $396 \mathrm{~Hz}(0.5)$

- For $\delta$ band distributed energy TRT has a bigger impact ( $60 \& 6$ times for left and right respectively), followed by EAE $(1.5 \& 0.5)$ and Binaural $396 \mathrm{~Hz}(0.5)$

- For $\theta$ band distributed energy TRT has a bigger impact (4 \& 1.5 times for left and right respectively), followed by EAE ( $1 \& 0.5)$ and Binaural $396 \mathrm{~Hz}(0.5)$

- For a band distributed energy TRT has a bigger impact ( $4 \& 1$ times for left and right respectively), followed by EAE $(1 \& 0.5)$ and Binaural $396 \mathrm{~Hz}(0.5)$

The data for Acoustic therapy condition of all channels measured and time / frequency domain parameters calculated (from the difference between session 4 and session 1) are described in the Annex AA. 


\subsubsection{Passive Mode (ERP) Data}

As described in 2.6.1.6 Passive mode, patients with EC were EEG recorded. 50 trials were taken during two and a half minutes. Stimulus with duration of 1 second and an inter-stimulus interval of 2.5 seconds were used.

After removing the artifacts and noise, the Grand average ERP (GAv) was calculated for every channel based on the data from all patients per acoustic therapy (Delorme, et al., 2015). What it means is that, for example, channel 1 for Binaural $396 \mathrm{~Hz}$ is calculated from the EEG data of channel 1 from all the 7 patients treated with Binaural $396 \mathrm{~Hz}$.

The GAv from all patients of the 16 Channels for the different acoustic therapies (Binaural $f_{0}$, Binaural $396 \mathrm{~Hz}$, TRT and EAE) for session 1 and 4 are shown in the Annex $\underline{A B}$. As it can be seen, some signals are small and the noise is higher than themselves or it does not exist any ERP in that channel. For several acoustic therapies, it happens in some of the channels such as $12,14,15$ or 16 .

Time-frequency analysis of spontaneous EEG and ERP data allows to investigate oscillatory brain activity. For instance, a P100 typically peaks at $100 \mathrm{~ms}$ and has a temporal width of around $50 \mathrm{~ms}$. If this ERP component is viewed as one half cycle of an oscillation, the $50 \mathrm{~ms}$ corresponds to an oscillation with a period of $100 \mathrm{~ms}$ (which is the equivalent of $10 \mathrm{~Hz}$ ) which corresponds to the alpha (8-13 Hz) band (Herrmann, et al., 2014).

Annex AC presents session 1 and 4 from a patient as an example per therapy of the independent components analysis and the principal components analysis calculated ${ }^{17}$ in the peak within the frequency domain. At first sight the evolution from session 1 to 4 for

- Binaural $f_{0}$ (frequency peak staying at $10 \mathrm{~Hz}$ ) from the third independent component onwards, the source location of the relative power is moving from occipital lobe (session 1) to frontal lobe (session 4)

- Binaural $396 \mathrm{~Hz}$ (frequency peak from $12 \mathrm{~Hz}$ in session 1 to $11 \mathrm{~Hz}$ in session 4) also moves from the third independent component onwards, from occipital lobe (session 1) to frontal lobe (session 4) but in a more focused manner through the frontal lobe.

- TRT (frequency peak staying at $9 \mathrm{~Hz}$ ) locates from positive power in the frontal lobe (session 1) to negative at the third independent component and positive parietal lobe at the fourth (session 4).

\footnotetext{
${ }_{17}$ Using EEGLAB (Delorme \& Makeig, 2004; Delorme \& Makeig, 2012; Krol, et al., 2018; Lopez-Calderon \& Luck, 2014).
} 
- EAE (frequency peak from $9 \mathrm{~Hz}$ in session 1 to $8 \mathrm{~Hz}$ in session 4) changes from right occipital, frontal and left parietal lobes (session 1) to frontal lobe and central sulcus (session 4) at the first three independent components.

Annex AD exposes the graphs of the location for the main ERP representation within the time domain (100, 200, 300 and $400 \mathrm{~ms})$ from session 1 and 4 of a patient as an example per therapy.

- Binaural $f_{0}$ delays the ERP from session 1 to 4 with a similar amplitude

- Binaural $396 \mathrm{~Hz}$ has a similar ERP latency from session 1 to 4 with an increase of the amplitude (more than $20 \mu \mathrm{V}$ P1-N1 peak to peak)

- TRT delays the ERP from session 1 to 4 with an decrease of the amplitude (around $-4 \mu \mathrm{V}$ P1-N1 peak to peak)

- EAE has a similar ERP latency from session 1 to 4 with an increase of the amplitude (around $-4 \mu \mathrm{V}$ P1-N1 peak to peak)

As the previous Resting and Acoustic therapy condition, common statistical features for time and frequency domain analysis are calculated for the GAv.

- frequency domain analysis (Annex $U)$

o from the FFT of GAv: P1 $\left(X_{m f}\right), P 2, P 3, P 4, P 5\left(M N F=X_{f c}\right), P 6, P 7\left(X_{r m s t}\right)$, P8, P9, P10, P11, P12, P13, P14, MDF and Power Spectrum deformation.

- Separation of bands $(\delta, \theta, \alpha, \beta, \gamma)$ is applied and PSD calculated for the total of the signal and every of those bands. Moreover, in order to assess the spectral energy, the sum of the square of the differences between the samplers is calculated.

- time domain analysis (Annex T)

- Mean, MeanAbs, Median, MMAV1, MMAV2, STD, VAR

- Root, RMS, IEEEG, SSI or WL.

- Crest, Skewness, Kurtosis, Clearance, Shape, and Impulse.

- Additionally, the amplitude and latency of the following ERP are calculated: P1, N1 and P2-P3 ${ }^{18}$

The time and frequency domain parameters calculated from the difference between sessions 4 and 1 of the GAv per therapy are displayed in the Annex AF. A summary of the more representative parameters are shown in Annex AE for the channels located in the frontal and temporal lobes.

In order to compare those parameters, it has been followed what was described in 2.5.2 Evoke Activity (ERPs) where N1 evoke activity characterizes neural deficit, P3 evoke

\footnotetext{
${ }^{18}$ Some of the different acoustic therapies ERP do not show a clear P2 or P3 ERP around 200 or $300 \mathrm{~ms}$ respectively. That is why it has been calculated either one or the other to compare among themselves (session 4 - session 1).
} 
activity is linked to the cognitive function, Power of $\delta$ band associated to tinnitus distress, Power of $\alpha$ band for tinnitus attention, Power of $\beta$ band to emotional distress and Power of $\gamma$ band connected to tinnitus perception and loudness.

- $\mathrm{P} 1$ amplitude is higher for Binaural $396 \mathrm{~Hz}$ (from 0.5 to 1 times from session 1 to 4) followed by Binaural (similar amplitude) to TRT and EAE (reduced by $\approx 0.5$ )

- $\mathrm{N} 1$ amplitude is higher in TRT (from 0.5 to 1 times from session 1 to 4 ) followed by Binaural (0 to 0.5) EAE (0 to 0.3 ) and Binaural $396 \mathrm{~Hz}(-0.15$ to 0.15$)$

- $\mathrm{N} 1$ latency is a bit longer for Binaural $(\approx 0.5)$ followed by TRT $(\approx 0.1)$, EAE and Binaural $396 \mathrm{~Hz}(\approx 0)$

- For TRT and EAE P2 is delay and close to $300 \mathrm{~ms}$. In regards difference between session 4 and 1 of

- P2 amplitude increases $(\approx 1)$ for Binaural in the frontal lobe followed by Binaural $396 \mathrm{~Hz}$ (left side) and decreases $(\approx 0.1)$ for TRT and EAE. For the temporal lobe the amplitude increases for Binaural followed by Binaural $396 \mathrm{~Hz}$ (left side), EAE $(\approx 0.1)$ and decreases $(\approx 0.1)$ for TRT (right side)

- P2 latency increase $(\approx 0.2)$ for Binaural $396 \mathrm{~Hz}$ followed by $\mathrm{EAE}(\approx 0.1)$, TRT and Binaural.

- $\delta$ band: Binaural improves the power (up to 4 times) followed by TRT $(\approx 2)$, EAE and Binaural $396 \mathrm{~Hz}$

- $\theta$ band: TRT improves (4 times) followed by Binaural $(\approx 2)$, EAE and Binaural $396 \mathrm{~Hz}$.

- $\quad$ band: TRT improves (10 to 25 ) followed by Binaural (2 to 5), EAE and Binaural $396 \mathrm{~Hz}$ which reduces the power in the temporal lobe $(\approx 0.5)$

- $\beta$ band: Binaural improves left side of frontal and temporal lobe (2 \& 5 times respectively) followed by the decrease $(\approx 0.2-0.8)$ of Binaural $396 \mathrm{~Hz}$, EAE and TRT.

- $\gamma$ band: Binaural improves left side of frontal and temporal lobe (4 \& 2 times respectively) followed by the right side of Binaural $396 \mathrm{~Hz}(1 \& 3)$ and the decrease $(\approx 0.5-0.8)$ of EAE and TRT. 


\section{CONCLUSIONS}

"Music creates order out of chaos: for rhythm imposes unanimity upon the divergent, melody imposes continuity upon the disjointed, and harmony

imposes compatibility upon the incongruous"

Yehudi Menuhin (1916-1999), violinist and conductor 


\subsection{INTRODUCTION}

Tinnitus is more than a background auditory sensation: It also has emotional and cognitive components and a strong placebo dependency.

It has been well established that chronic and refractory tinnitus is the result of a tonotopic cortical reorganization owing to neural synchrony abnormalities in the auditory cortex, the limbic system or the hippocampus.

On the other hand, sound is the propagation of mechanical waves that cause vibrations in the matter, and which can even make it vibrate at different frequencies. So, it is possible to hypothesize that acoustic-based treatments could reverse neural synchrony abnormalities in the auditory cortex that are generating tinnitus by finding the appropriate vibration frequency.

Unfortunately, treatment recommendations have not yet transformed standards in the tinnitus field so far. Indeed, recent systematic reviews of published clinical trials aiming at evaluating tinnitus therapeutic interventions have shown that reporting is still flawed by poor methodology and poor reporting (Londero \& Hall, 2017).

Up to now, a wide variety of acoustic therapies has been proposed to alleviate tinnitus. In line with previous studies, the most effective sound-based treatments were selected (TRT, EAE and Binaural), and were tested in patients with chronic and refractory tinnitus for two months.

Patients were aware of the procedure and signed a consent form. Acoustic therapies were personalized for each patient and their head physician followed-up the experiment. Patients were instructed to use their therapies every day for one hour, at any time of the day. Assistance during the experiment was provided.

The follow-up treatment was based on CIA and HADS questionnaires where psychological monitoring can reveal emotional and behavioural irregularities related to tinnitus. On top of that, EEG measurements were introduced to avoid the trial-error subjective evaluation, introduce an effective quantification of the acoustic therapy effects and put in place a more rigorous physiological output were repeatability and reproducibility are presented.

EEG is the most widely used method to monitor brain activity for diagnosing brain disorders, and predicting treatment outcome success. Up to now, the major EEG feature under study is neural oscillations. These neural oscillations are modulated by the internal communication of neural nodes (local synchrony) and the intercommunication among those neural nodes (global synchrony). As subjective tinnitus is result of increased neural synchrony and SFRs in the auditory system, EEG analysis is outlined to be an objective method to assess the effects of acoustic therapies to treat tinnitus. EEG signal processing offers to investigate: internal communication in neural 
nodes (absolute and relative power), inter-communication of neural nodes (coherence), time-frequency analysis with high resolution in both low and high frequencies (Wavelet analysis), rate at which neural information is being produced (entropy), genesis of abnormal neural synchrony on the basis of biophysical assumptions (source localization) or statistical evaluations (PCA and ICA), and level of neural synchrony over time (ERD/ERS maps).

At present, no reliable EEG-based methods have been found. In fact, some authors (Pierzycki, et al., 2016) consider that EEG is not an effective way to study tinnitus effects. On this evidence, several EEG evaluations have been proposed in this thesis: from no specific auditory stimulation (resting condition), to auditory pattern recognition (active mode). This set-up will also lead us to propose an efficient EEG-based methodology to study tinnitus effects.

The main purpose of this thesis is to measure beneficial and side effects of on-the-spot acoustic therapies to treat chronic and refractory tinnitus, and thus designing sound combination treatments with the final goal of improving the life quality of the Tinnitus patients. The most significant findings are hereunder discussed.

\subsection{SUBJECTIVE ANALYSIS CONCLUSIONS}

The biggest issue faced during the measurements and analysis was that all patients did not have a discipline where they listened one hour therapy every day, as recommended. The transfer of responsibility is affected on the mood and patient's ability to manage the tasks involved (Richardson, et al., 2014).

For example, there was a patient recovering from cancer and she had a big improvement in her Tinnitus. She was mixing both regular exercises with the therapy (so she forced herself to do it every day) and she self-reported one of the biggest achievements (using binaural therapy).

According to Brown and Bussell (2011) regarding the treatment of chronic illnesses, approximately $50 \%$ of patients do not take their medications as prescribed, so their full benefits and efficiency are often not realized.

Moreover, some patients were nervous only with the fact of being in the process of receiving the acoustic therapy. Others, after giving them some attention about his/her problems, it could improve because he/she felt released and relaxed talking about it, what it could be considered as a Placebo effect. In both cases, it was radically affecting the stress and linked to that the Tinnitus itself.

Some medical studies have shown that patients who are given a placebo remedy, experience improvement of their symptoms, such as cough, cold, headache, dizziness or anxiety (Lahiri \& Duncan, 2007; Jenkins, 2001; Reinhardt \& Lange, 1982). 
Those are some of the reasons because subjective data need to be cross-checked with some objective information that it will explained in 3.3 Objective Analysis.

Overall, it is important to have in mind that only positive effects were statistically conclusive, but negative ones were not. So, caution must be applied with the discussion about undesirable effects since those were not significant. Additionally, the majority of patients reported no effects. There is, therefore, abundant room for further progress in enhancing the design of acoustic therapies according to the discoveries of the present work.
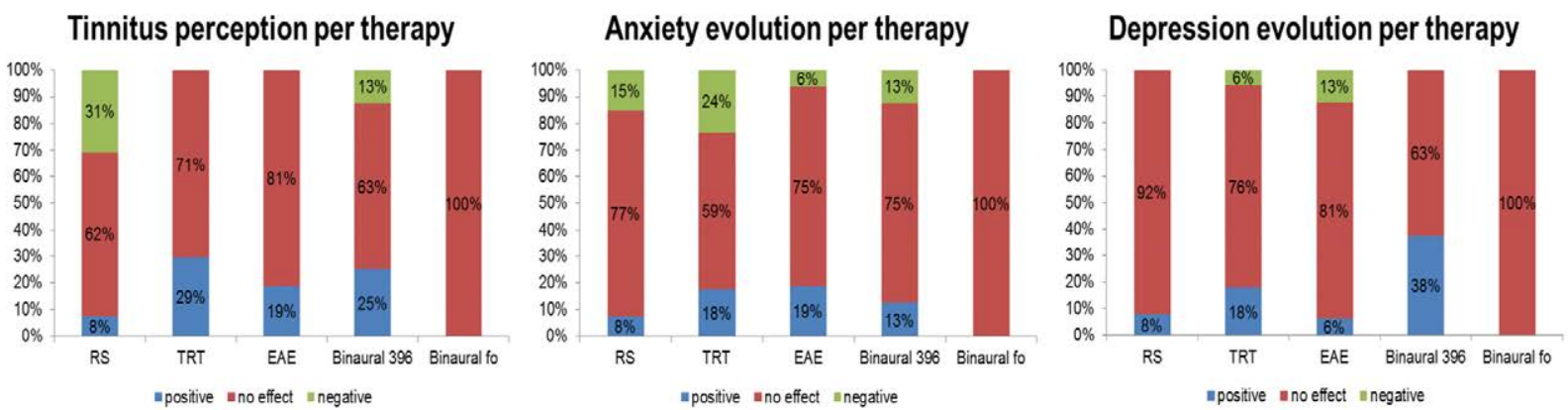

Figure 14: Subjective analysis summary per therapy after two-month treatment in line with CIA.EVO-based monitoring

Nevertheless, based on the results obtained from these patients' measurements shown in the figure 14 and what it has been discussed previously along the 3.2 Subjective Analysis, it can be concluded the following:

\subsubsection{RELATIVELY EFFECTIVE ACOUSTIC THERAPY: TRT}

The current study found that TRT tends to be the most effective acoustic therapy (in comparison with Binaural, EAE and RS) to reduce tinnitus perception, and to relieve anxiety and stress due to tinnitus after a two-month treatment.

TRT had been previously recognized as an effective method to reduce tinnitus perception, and even, updated versions has been tested (e.g., narrow, mixed and broad band noise). In general, it has been observed (Lee, et al., 2018; Bauer, et al., 2017; Kim, et al., 2014) that

- tinnitus perception was significantly reduced after the ninth week of a TRT-based treatment

- tinnitus suffers with hearing loss reported improvements at the third week of the treatment

- broad band noise TRT seems to be the most effective version

However, it was not specified whether TRT helped to control anxiety and stress. Only Westin et al. (2011) compared acceptance and commitment therapy (ACT) with TRT, 
and reported that TRT helped to get sleep and relieve anxiety, but ACT was much more effective. In contrast to that result (Westin, et al., 2011), this study showed that TRT aggravated anxiety in a large portion of the sample (18\%): A result that is similar to the patients who reported reduction of tinnitus perception (29\%). On this evidence, TRT is an acoustic treatment that may be not recommended for individuals who suffers from anxiety disorders.

\subsubsection{MODERATE ACOUSTIC THERAPY: BINAURAL $396 \mathrm{HZ}$ AND EAE}

On one hand, Binaural $396 \mathrm{~Hz}$ is the best therapy used to ameliorate the depression. Moreover, in particular cases, it is still a proper option to relieve anxiety and improve tinnitus perception when

- the initial CIA is higher, Binaural $396 \mathrm{~Hz}$ has a similar effect produced than TRT for tinnitus perception reduction

- the initial HADS-A is higher, Binaural $396 \mathrm{~Hz}$ behaves like EAE.

These findings may be somewhat aligned by the relatively high number of patients $(67 \%)$ who reported to gain a growing tinnitus perception in Binaural therapy groups. A possible explanation for Binaural side effects might be that binaural beats alter states of consciousness (Petrovich, 2018); as a result, some patients could be raising the level of awareness of their tinnitus reflected as a greater perception.

On the other hand, EAE is still a useful acoustic therapy to reduce tinnitus perception, an adequate choice to decrease depression and the most efficient one to improve anxiety.

It is well established that enriched acoustic environments after noise trauma prevents tonotopic cortical reorganization what is the cause of tinnitus (Noreña \& Eggermont, 2005). If $E A E$ can abolish neural signs of tinnitus, this might explain that patients in the EAE group exclusively reported reduction of tinnitus perception. Also, it has been found that enriched acoustic environments may increase tinnitus annoyance and depressive feelings, what may explain the increase of stress reported by patients in the EAE group of this study (Vanneste, et al., 2013).

\subsubsection{NOT RECOMMENDED TREATMENT: RS AND BINAURAL $F_{0}$}

One unanticipated finding has been that RS could bring harmful effects, particularly in term of tinnitus perception and anxiety control, whereas positive effects were negligible. With respect to music therapy for chronic tinnitus, there are still very controversial findings. In 2012, Argstatter et al. found that Heidelberg model of music therapy was successfully applied to treat patients with tonal tinnitus (Argstatter, et al., 2012). In 2013, Vanneste et al. showed that music therapy compensating for hearing loss was not beneficial in suppressing tinnitus, and over compensating hearing loss worsened tinnitus (Vanneste, et al., 2013). In 2014, Pape et al. demonstrated that tailor-made 
notched music in non-musician tinnitus suffers could reduce tinnitus perception (Pape, et al., 2014), contrary to the work of Stein et al. (2016), who found that such treatment did not reduce tinnitus perception and induce temporal high levels of distress (Stein, et al., 2016). Although it is common practice to recommend music therapy to tinnitus suffers, it should be taken with caution since certain kind of music as the one used in this study might considerably worsen tinnitus condition.

Furthermore, Binaural $f_{0}$ has no effect at all though the general reaction from the patients is an improvement of tinnitus perception, as explained before.

For that purpose, frequency adjustment may be a solution to reduce the number of patients who could present side effects. It is proposed to redesign Binaural $396 \mathrm{~Hz}$ according to individual alpha frequencies, instead of arbitrary and generic alpha values $(10 \mathrm{~Hz})$. This individual parameter can be estimated according to the brain-body coupling theory proposed by Klimesch (2013), who proposed to calculate neural frequency bands in line with the heart rate.

\subsection{OBJECTIVE ANALYSIS CONCLUSIONS}

Following what it was explained in previous chapters (Geller, et al., 2014) eye closure was increasing the effect of acoustic therapies during the auditory retraining due to the diminution of high frequency brain oscillations detecting easily the neural synchronization and taking into consideration that tinnitus perception is an internal attentional process and involves less interaction with the environment (external attentional process).

Other general remark is that it exists a different performance among right and left side in particular when the acoustic therapies condition is applied. It might be produced due to a decompensation between the number of left and right side tinnitus patients assessed for every acoustic therapy.

EEG mapping of tinnitus perception during acoustic therapy should reveal if there was any neural synchrony modification as a result of the treatment, in comparison with resting condition when this was not received yet.

The use of auditory stimulus based on the acoustic therapy should standardize the evoke response in each group. As already said

- N1 Tinnitus patients' performance has less amplitude and shorter latency.

- P3 Tinnitus patients have higher latency and less amplitude. On the other hand, P2 is delay (shorter latency)

- Tinnitus brain oscillatory activity

- Generally, decreases in the alpha band, and increases in delta and gamma bands. 
- Abnormally found on the frontal lobe (characterized by the channels 1 and 2) which correspond to deficiency of emotions and interpretation attributed to the stress caused by the Tinnitus.

- Irregularly on the temporal lobe (channels 3 and 7) associated to decoding and perception, and linked to concentration.

After the analysis of the previous objective data, the conclusion is the following:

\subsubsection{RELATIVELY EFFECTIVE ACOUSTIC THERAPY: BINAURAL F $F_{0}$ AND $396 \mathrm{HZ}$}

Tinnitus patients using Binaural therapy have an impressively much better response than other therapies regarding the resting condition in terms of reduction of stress and improving concentration from the average behaviour of the session 4 against the session 1.

Apart of that, Binaural $f_{0}$ has the highest multiplication for the signal power in total and in particular for the $\delta$ band (tinnitus distress) and the second best of a band (tinnitus attention).

In terms of evoke activity, the source is located in the frontal lobe, the latency is slightly shorter for $\mathrm{N} 1$ and $\mathrm{P} 3$ and the amplitude increase in $\mathrm{P} 1$ and $\mathrm{P} 3$ while staying for $\mathrm{N} 1$.

Binaural $396 \mathrm{~Hz}$ with a reasonable performance in its parameters, is more focused in the central frontal lobe and with an even more amplitude in P1.

\subsubsection{MODERATE ACOUSTIC THERAPY: TRT}

TRT resting condition (EC) supports positively the reduction of stress and tinnitus attention, though with EO the behaviour is worse (frontal) or keep no difference (frontal).

This acoustic therapy is the one with a second biggest total energy and in particular the first for $\alpha$ band and second for $\delta$ band.

The principal components for the evoke activity are located in the frontal and parietal lobe. With the exception of $\mathrm{N} 1$ where TRT has the highest amplitude, the remaining ERPs have no increase in amplitudes or difference between latencies.

\subsubsection{NOT RECOMMENDED TREATMENT: RS AND EAE}

Despite not having enough data from the patients regarding evoke responses, it might be assessed that RS does not have an effect regarding reducing stress or conducting better concentration because there is no big changes in the source of power located neither big differences among any of the parameters in time or frequency domain.

EAE resting condition (EC) maintains almost same level of performance. On the other hand, with EO is much worse. This therapy stays with a constant power and the evoke 
activity is located in the frontal and central parietal lobe with almost no increase in any of the amplitudes or difference between latencies.

\subsection{SUBJECTIVE AND OBJECTIVE ANALYSIS CONCLUSIONS}

It is important to keep in mind that tinnitus frequency response curve is subjectively obtained in humans (Roberts, et al., 2006), and it would depend on patients' perception to establish auditory stimulus, what in turn could produce subjective outcomes, that is why objective results are required to understand the behavior of the patients.

Sympathetic activation is required for attention. Separate studies have shown that meditation improves attention and facilitates the processing of information in the auditory association cortex. Some symptoms are the decrease of the average heart rate, increase in the P3 peak amplitude, reduction in peak latency (Telles, et al., 2018) or decrease in the peak latency of the P2 component (Telles, et al., 2015). Until certain point, those acoustic therapies could be seen as a way to force and empower meditation or at least a resting state.

Active listening to one's own tinnitus leads to increased tinnitus distress, presence and loudness while neural correlates in the EEG data are missing. On the other hand, invariant resting state brain activity in tinnitus sufferers may not be altered by attention to the tinnitus percept (Neff, et al., 2019) what means that even if the use of the acoustic therapy is able to concentrate even more the patient attention to the tinnitus, the objective performance will stay the same.

To summarize the results of the subjective and objective analysis from the data assessed during 2 months of the different sessions where tinnitus patients applied a specific acoustic signal, the best performance therapies are:

- Binaural $f_{0}$

- with a subjectively not recommended position because apparently didn't have any effect in the average evolution of the patients' sessions assessment

- surprisingly, it has the best response during the resting condition in terms of reduction of stress (frontal lobe) and improving concentration (temporal lobe), ERP (P1 and P3) amplitude increase, tinnitus distress ( $\delta$ band) and tinnitus attention ( $\alpha$ band)

- Binaural $396 \mathrm{~Hz}$

- chosen subjectively for decreasing depression and as a proper option to relieve anxiety and improve tinnitus perception 
0 it has a reasonable performance (less than Binaural $f_{0}$ ) in its temporal and frequency domain parameters, with an evoke activity more focused in the central frontal lobe and with the highest amplitude in P1.

- TRT

- subjectively has the better behavior for reducing tinnitus perception with the contraindication of not using it for anxiety disorder patients

- objectively its resting condition with EC supports positively the reduction of stress and tinnitus attention, the total energy is considerably big and in particular the best $\alpha$ band (tinnitus distress), the evoke activity is located in the frontal and parietal lobe with $\mathrm{N} 1$ as highest (negative) amplitude.

On the other hand, the not recommended acoustic therapies assessed from the data during these 2 months are:

- $\mathrm{EAE}$

- subjectively it is still a useful acoustic therapy to reduce tinnitus perception but suggested to avoid it on tinnitus suffers with anxiety and stress disorders.

- objectively, it stays with a constant power and the evoke activity is located in the frontal and central parietal lobe with almost no increase in any of the amplitudes or difference between latencies.

- RS

- music therapies should be recommended with caution since they could worsen tinnitus condition too.

- objectively, no big change in the source of power located neither big difference among any of the parameters in time or frequency domain. 
CONTRIBUTIONS 
Below it is shown the main contributions of this thesis:

- Bibliographic compilation corresponding to 281 documents, such as scientific articles and books. Among others, those references cover topics like tinnitus, acoustic therapies with a special interest in binaural beats, neurological and auditory processing, subjective and objective analysis, cognition and stress management.

- A clinical protocol has been established and implemented for patients suffering from refractory and chronic tinnitus who have being treated with acoustic therapies. The protocol displays an objective methodology to treat tinnitus with acoustic therapies looking into the neural electrical activity based on EEG evaluation cross checking the data with subjective questionnaires.

- Tinnitus frequency response curve is subjectively obtained in humans and we would depend on patients' perception to establish auditory stimulus, what in turn could produce subjective outcomes.

- Trial-error protocol might delay the patient healing, or even could deteriorate the patient condition. Establish an objective evaluation of acoustic therapies based on EEG improve the conventional trial-error procedure.

- EEG evaluation was not used for resting condition and evoked activity on studying tinnitus nature, but not for studying acoustic therapy effects.

- Evaluation of therapy effect (independent variable) has been achieved rather than tinnitus perception (per patient) per se.

- 68 random patients from the INR were recruited. They took part voluntarily, unsalaried and passed the four different measurement stages (at the beginning, at one week, at five weeks and at eight weeks of treatment) apart of listening one hour of their personalized acoustic therapy per day.

- Three acoustic therapies were created besides of the Relaxing Signal group.

o TRT and TEAE which have been demonstrated already good results for Tinnitus treatment.

- Binaural beats with not many references for tinnitus but for reducing stress and anxiety. The parameters selected were frequency rate: $10 \mathrm{~Hz}$, carrier signal: tinnitus frequency $\left(f_{0}\right)$ or $396 \mathrm{~Hz}$.

- Hypothesis turned into facts: Therapies have produced perceptual and cognitive changes. Electrophysiological changes of brain frequencies were evaluated and observed before and after the acoustic stimulation.

- Binaural therapy has the best response during the resting condition in terms of reduction of stress (frontal lobe) and improving concentration 
(temporal lobe), ERP (P1 and P3) amplitude increase, tinnitus distress ( $\delta$ band) and tinnitus attention ( $\alpha$ band).

- TRT supports positively the reduction of stress and tinnitus attention. The total energy is considerably big and in particular the best $\alpha$ band (tinnitus distress), the evoke activity is located in the frontal and parietal lobe with $\mathrm{N} 1$ as highest (negative) amplitude.

- EAE stays with a constant power and the evoke activity is located in the frontal and central parietal lobe with almost no increase in any of the amplitudes or difference between latencies.

- RS should be recommended with caution since they could worsen tinnitus condition, according to the subjective feedback. It has no big change in the source of power located neither big difference among any of the parameters in time or frequency domain. 


\section{QUALITY INDICATORS}


Auris Nasus Larynx 45 (2018) 6-12

Contents lists available at ScienceDirect

Auris Nasus Larynx

journal homepage: www.elsevier.com/locate/anI

\section{In-ear medical devices for acoustic therapies in tinnitus treatments, state of the art}

David Ibarra $^{\mathrm{a}, \mathrm{b}, *}$, Francisco Tavira-Sanchez ${ }^{\mathrm{c}}$, Manuel Recuero-Lopez ${ }^{\mathrm{c}}$, Brian W. Anthony ${ }^{\mathrm{b}}$

${ }^{a}$ Tecnológico de Monterrey, Escuela de Ingeniería y Ciencias, Campus Mexico City, Calle del Puente 222, Ejidos de Huipulco, Tlalpan, 14380 Mexico D.F.. Mexico

'Laboratory for Manufacturing and Productivity, Massachusetts Institute of Technology, 77 Massachusetts Avenue, Cambridge, MA 02139, USA

${ }^{\mathrm{C}}$ Grupo de Investigación en Instrumentación y Acústica Aplicada (I2A2), Universidad Politécnica de Madrid, Carretera de Valencia km. 7. 28031 Madrid, Spain

A R TICLE INFO

Article history:

Received 6 January 2017

Accepted 28 March 2017

Available online 21 April 2017

\section{Keywords:}

Medical device

Hearing aid

Acoustic therapy

Tinnitus

\begin{abstract}
A B S T R A C T
Cochrane reviews indicate there is very limited support for all forms of sound therapy and cognitive behavioral therapy has the strongest support. American Academy of Otolaryngology (AAO) recently published some guidelines which recommends Cognitive Behavioral Therapy (CBT) for tinnitus intervention, and only indicates that sound therapy should be considered an "option" for intervention. Nevertheless, acoustic therapy could lead to cause changes in the tinnitus perception and has been appreciated by the affected people for years. In the last decades, the use of sound or sound enrichment has become a central part of many tinnitus management programs used by audiologists, whether the intention was to mask tinnitus, suppress tinnitus, or interrupt the tinnitus generating neural activity.

Several acoustic therapies have been developed and implemented in the last 40 years, but how can we determine which one is the most effective? We can determine the effects based on the results reported in many research studies, but in those studies are many factors that differ from one study to another, like in-ear medical devices used to apply acoustic therapy for tinnitus treatment.

In this article, we review and analyze the different types of in-ear medical devices used in the most recently acoustic therapies in treatments against tinnitus, allowing us to identify the pros and cons. By our analysis, an optimal medical device could be characterized to enhance the application of acoustic therapies and in consequence the global results of the sound therapies that already exist. In this review, it was considered acoustic therapies, the technology implemented in medical devices and the clinical needs.
\end{abstract}

(1) 2017 Elsevier B.V. All rights reserved.

\section{Introduction}

Tinnitus is known to affect between $5 \%$ and $15 \%$ of the population [1]. About half of the people with tinnitus are concerned about it, and it is reported that about $2 \%$ indicate that

\footnotetext{
* Corresponding author.

E-mail address: david.ibarra (@i) itesm.mx (D. Ibarra).

http://dx.doi.org/10.1016/j.anl.2017.03.020

0385-8146/@ 2017 Elsevier B.V. All rights reserved.
}

tinnitus significantly affects their life. Tinnitus may have a direct effect on a person's emotional wellbeing, their hearing, and ability to sleep and to concentrate. These in turn impact basic life functions such as socialization and relaxation [2]. In severe cases, it can interfere with the individual's ability to perform adequately on the job, or contribute to psychological disorders such as depression, suicide ideation, posttraumatic stress disorder, anxiety, and anger [3].

\footnotetext{
${ }^{19}$ To download the paper, please click in https://www.ncbi.nlm.nih.gov/pubmed/28438439
} 
Electroencephalographic evaluation of acoustic therapies for the treatment of chronic and refractory tinnitus ${ }^{20}-J C R(Q 2)$

\title{
Electroencephalographic evaluation of acoustic therapies for the treatment of chronic and refractory tinnitus
}

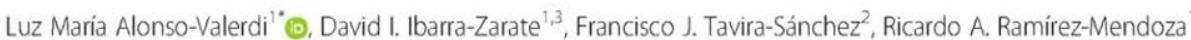
and Manuel Recuero ${ }^{2}$

\begin{abstract}
Background: To date, a large number of acoustic therapies have been applied to treat tinnitus. The effect that produces those auditory stimuli is, however, not well understood yet. Furthermore, the conventional clinical protocol is based on a trial-error procedure, and there is not a formal and adequate treatment follow-up. At present, the only way to evaluate acoustic therapies is by means of subjective methods such as analog visual scale and ad-hoc questionnaires.

Methods: This protocol seeks to establish an objective methodology to treat tinnitus with acoustic therapies based on electroencephalographic (EEG) activity evaluation. On the hypothesis that acoustic therapies should produce perceptual and cognitive changes at a cortical level, it is proposed to examine neural electrical activity of patients suffering from refractory and chronic tinnitus in four different stages: at the beginning of the experiment, at one week of treatment, at five weeks of treatment, and at eight weeks of treatment. Four of the most efficient acoustic therapies found at the moment are considered: retraining, auditory discrimination, enriched acoustic environment, and binaural.

Discussion: EEG has become a standard brain imaging tool to quantify and qualify neural oscillations, which are basically spatial, temporal, and spectral patterns associated with particular perceptual, cognitive, motor and emotional processes. Neural oscillations have been traditionally studied on the basis of event-related experiments, where time-locked and phase-locked responses (i.e., event-related potentials) along with time-locked but not necessary phase-locked responses (i.e., event-related (de) synchronization) have been essentially estimated. Both potentials and levels of synchronization related to auditory stimuli are herein proposed to assess the effect of acoustic therapies.

Trial registration: Registration Number: ISRCTN14553550. ISRCTN Registry: BioMed Central. Date of Registration: October 31st, 2017.

Keywords: Tinnitus, Acoustic therapy, Electroencephalography (EEG), Auditory perception, Neural oscillations
\end{abstract}

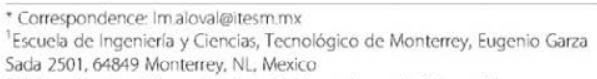

Bïowed Central

The Author(s). 2017 Open Access This article is distributed under the terms of the Greative Commons Attribution 4.0 intermational License (http//creativecommons.orc/licenses/ty/4.00), which permits unrestricted use, distribution, and reproduction in ary medium, provided you give appropriate crecit to the original author(s) and the source, provide a link to

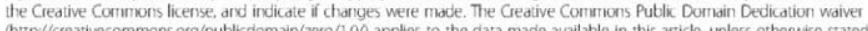

${ }^{20}$ To download the paper, please click in https://rdcu.be/3XcR 
FUTURE RESEARCH TOPICS 
1. As described in 3.1 Introduction where it was explained the Results of this thesis, the active measurements from the patients were not in the scope of the analysis and it will be treated afterwards, what means the study of induce response by event-related oscillations (EROs) and the EEG event-related (de)synchronization (ERD/ERS) which will allow to understand the effectiveness of the acoustic therapies to improve the perceptual and cognitive development.

2. In order to enhance the objective evaluation

- There are available more data to be analysed: all the channels located all over the scalp (not only frontal lobe) and from the 4 sessions (not only the different between the 4 and 1 ).

- It would be convenient and useful to increase the number of parameters taken such as: Breathing Rate, Blood pressure, Electrodermal Activity or Electrocardiography ${ }^{21}$

- I might be necessary to cluster standardized patient clinical history parameters such as: initial Tinnitus (CIA), Anxiety and Depression (HADS) perception, hearing loss, tinnitus frequency and intensity, tinnitus laterality, duration ${ }^{22}$ and age ${ }^{23}$.

- In general, EEG spatial resolution is a weakness and underdetermined ${ }^{24}$. A way to improve it is increasing the number of electrodes mounted (up to 128).

- It could be use a magnetoencephalography which has better spatial resolution and more sensitive to activity in more brain areas than EEG.

3. On the other hand, it still required to fine-tune the acoustic signal associated to a specific patient who will allow to boost his/her quality of life. Due to that reason other acoustic signals may be tested

- Changing the frequency rate or carrier signal from the Binaural therapy.

- Adding pink noise to the current or future Binaural signals.

- Using TPO, HFT, ADT or Neuromodulation described in $1.2 \mathrm{SoA}$

\footnotetext{
${ }^{21}$ Heart Rate Variability, Inter-beat (R-R) Interval, Peak Acceleration and Heart Rate

22 Tinnitus subjects with a longer history of tinnitus showed less variability of their auditory alpha activity which might be an indicator for reduced adaptability of the auditory cortex in chronic tinnitus. High tinnitus related distress patients are captured by their tinnitus significant more than in low distress patients (Delb, et al., 2008).

${ }^{23}$ It was observed than age-associated effects on N1 and N2 amplitudes and on N2 latency in both spatial conditions. Moreover, impaired allocentric performance in older subjects was associated with greater P2 amplitude and delayed P2 latency compared with younger subjects. The results suggest that elderly individuals present with deficits when spatial orientation relies on allocentric representation, and are associated with age-related deficits inspatial attention (Lithfous, et al., 2014).

${ }^{24}$ Adjamian (2014) commented that the determination of signal sources is complex and inaccurate due to EEG nature. It cannot specify a location for ERPs, it can only expect to find the information over frontal, central and temporal regions, where auditory ERPs are commonly found.
} 
- Practising with new technics such as isochronic tones which are regular beats that are being turned on and off rapidly. It may be utilised alongside the Binaural signal and the rhythm ${ }^{25}$ will have to be characterized.

4. Instead of relying on previous state of the art studies and researches conclusions, a specific control group (people without Tinnitus) would be a better way to crosscheck and match the acoustic signals applied with the patients.

5. For a better comprehensive tinnitus diagnosis, a multidisciplinary approach is recommended. A further step should be mixing acoustic therapies with a defined diet or some Tinnitus drugs such as Piracetam (Malykh \& Sadaie, 2010; Ravishankar, 2017) or Mind Lab Pro® nootropics (Mindlabpro, 2018).

6. A smartphone application could be developed in order to

o develop a monitoring system to record the frequency of use of the acoustic therapy, to provide reminders about the upcoming application, and even, to recommend a specific acoustic therapy in accordance to the current condition of the tinnitus suffer ${ }^{26}$

- select the most appropriate acoustic therapy in accordance with the clinical history of each patient.

- Scale up the number of patients getting "long term" measurements to define features such us the better exposition time to the therapy.

- Connect it to a Brain Computer Interface commercial device, for instance BrainWear ${ }^{\circledR}$ from Emotiv or Muse ${ }^{\mathrm{TM}}$ headband ${ }^{27}$.

- Apply other technologies with the acoustic therapies like Augmented Reality, Virtual Reality or Mixed Reality to get "sight" as an additional sense.

- Establish a new protocol to standardize the type of patients, the general process, way of working and Self-Administration Errors awareness ${ }^{28}$.

\footnotetext{
${ }^{25}$ In this case, rhythm is consider the space between silence and sound

${ }^{26}$ As was found, each therapy has cons and pros what could be exploited to select the most effective treatment at specific times.

${ }^{27}$ More info at www.emotiv.com and www.choosemuse.com

${ }^{28}$ Uselton et al. (2010) explain how to prevent Self-Administration Error in their book.
} 


\section{BIBLIOGRAPHIC REFERENCES}

According to ISO 690:2010 norm ${ }^{29}$ 
ACTIONONHEARINGLOSS. Hearing Loss is major public health issue that affects more than 11 million people across the UK [online]. United Kingdom, 2016. [viewed 8 April 2017] Available from: https://www.actiononhearingloss.org.uk/supporting-you/policyresearch-and-influencing/research/hearing-matters.aspx.

ADAMCHIC, I., TOTH, T., HAUPTMANN, C. and TASS, P. A.. Reversing pathologically increased EEG power by acoustic coordinated reset neuromodulation. Human Brain Mapping. 2014, 35(5), pp. 2099-2118.

ADJAMIAN, $P$. The application of electro-and magneto-encephalography in tinnitus research-methods and interpretations. Frontiers in Neurology. 2014, 5(228), pp. 1-24.

ALA, T. S., AHMADI-PAJOUH, M. A. and NASRABADI, A. M. Cumulative effects of theta binaural beats on brain power and functional connectivity. Elsevier - ScienceDirect - Biomedical Signal Processing an Control. 2018, vol. 42, pp. 242-252.

ALTIN, C. and ER, O. Comparison of Different Time and Frequency Domain Feature Extraction Methods on Elbow Gesture's EMG. European Journal of Interdisciplinary Studies. 2005, 2(3).

ANDERSON, W. T. The Dalcroze Approach to Music Education: Theory and Applications. General Music Today. 2012, 26(1), pp. 27-33.

ANDERSSON, G. et al. Regional cerebral blood flow during tinnitus: a PET case study with lidocaine and auditory stimulation. Acta Otolaryngo. 2000, vol. 120, pp. 967-972.

ANNUNZIATA, M. A., MUZZATTI, B. and ALTOE, G. Defining Hospital Anxiety and Depression Scale (HADS) structure by confirmatory factor analysis: a contribution to validation for oncological settings. Annals of Oncology. 2011, vol. 22, pp. 2330-2333.

APARECIDA DE AZEVEDO, A., MELLO DE OLIVEIRA, P., GOMES DE SIQUEIRA, A. and RODRIGUES FIGUEIREDO, R. A critical analysis of tinnitus measuring methods. Brazilian Journal of Otorhinolaryngolog. 2007, vol. 3, p. 73.

APEDH, P., Fibonacci y Phi en la Música. [online]. Spain, 2014. [viewed 28 April 2017] Available from: http://natural432hz.blogspot.fr/2014/04/fibonacciy-phi-en-la-musica.html

ARGSTATTER, $\mathrm{H}$. et al. Long-term effects of the "Heidelberg Model of Music Therapy" in patients with chronic tinnitus. International journal of clinical and experimental medicine. 2012, 5(4), p. 273.

ARNOLD, W. et al. Focal metabolic activation in the predominant left auditory cortex in patients suffering from tinnitus: a PET study with [18F] deoxyglucose. ORL J. Otorhinolaryngol. Relat. Spec. 1996, vol.58, pp. 195-199.

ARYAA, N. K., SINGHA, K., MALIKA, A. and MEHROTRA, R. Effect of Heartfulness cleaning and meditation on heart rate variability. Indian Heart Journal. 2018.

ATTIAS, J., BRESLOFF, I., FURMAN, V. and URBACH, D. Auditory event related potentials in simulated tinnitus. Journal of Basic and Clinical Physiology and Pharmacology. 1995, 6(2), pp. 173-184. 
ATTIAS, J., FURMAN, Z., SHEMESH, Z. and BRESLOFF, I. Impaired brain processing in noise-induced tinnitus patients as measured by auditory and visual event-related potentials. Ear and Hearing. 1996, 17(4), pp. 327-333.

ATTIAS, J., URBACH, D., GOLD, S. and SHEMESH, Z. Auditory event related potentials in chronic tinnitus patients with noise induced hearing loss. Hearing Research. 1993, 71(1), pp. 106-113.

BAGULEY, D., ANDERSSON, G., MCFERRAN, D. and MCKENNA, L. The Jastreboff Neurophysiological Model, in Tinnitus: A Multidisciplinary Approach. West Sussex, UK: John Wiley and Sons, Ltd., 2013.

BAGULEY, D. M. Hyperacusis. J. R. Soc. Med. 2003, vol. 96, pp. 582-585.

BAGULEY, D., MCFERRAN, D. and HALL, D. Tinnitus. Lancet. 2013, vol. 382, pp. 1600-1607.

BALKENHOL, T., WALLHÄUSSER-FRANKE, E. and DELB, W. Psychoacoustic Tinnitus Loudness and Tinnitus-Related Distress Show Different Associations with Oscillatory Brain Activity. PlosOne. 2013, 8(1), p. e53180.

BALTUSSEN, R. et al. Costs of screening children for hearing disorders and delivery of hearing aids in China. BMC Health Serv Res. Apr ; 9():64. 2009, vol. 16, pp. 9-64.

BASTIAANSEN, M., MAZAHERI, A. and JENSEN, O. Beyond ERPs: Oscillatory Neuronal Dynamics. En: E. S. K. and. S. J. Luck, ed. The Oxford Handbook of EventRelated Potential Components. China: Oxford University Press, 2012, pp. 1-21.

BASTIEN, C. H., VALLIERES, A. and MORIN, C. M. Validation of the Insomnia Severity Index as an outcome measure for insomnia research. Sleep Medicine. 2001, 2(4), pp. 297-307.

BAUER, C. A., BERRY, J. L. and BROZOSKI, T. J. The effect of tinnitus retraining therapy on chronic tinnitus: a controlled trial. Laryngoscope investigative otolaryngology. 2017, 2(4), pp. 166-177.

BECHER, A. K. et al. Intracranial electroencephalography power and phase synchronization changes during monaural and binaural beat stimulation. European Journal of Neuroscienc. 2014, 41(2), pp. 254-263.

BECH, P. and WERMUTH, L. Applicability and validity of the Major Depression Inventory in patients with Parkinson's Disease. Nord. J. Psychiatry. 1998, vol. 52, pp. 305-309.

BECK, A. T., STEER, R. A. and BROWN, G. K. Beck depression inventory-II. San Antonio. 1996, 78(2), pp. 490-498..

BENEZON, R. O. Teoría de la musicoterapia. Madrid: Mandala, 2004

BJELLAND, I., DAHL, A. A., HAUG, T. T. and NECKELMANN, D. The validity of the Hospital Anxiety and Depression Scale: an updated literature review. Journal of Psychosomatic Research. 2002, 52(2), pp. 69-77. 
BLANKERTZ, B. et al. Neurophysiological predictor of SMR-based BCl performance. Neuroimage. 2010, 51(4), pp. 1303-1309.

BOND, M. et al. The effectiveness and cost-effectiveness of cochlear implants for severe to profound deafness in children and adults: a systematic review and economic model. Health Technol Assess. 2009, 13(44), pp. 1-330.

BOOSTANI, R., KARIMZADEH , F. and NAMI, M. Computer Methods and Programs in Biomedicine. ScienceDirect. 2017, vol. 140, pp. 77-91.

BOYD, M. and ELPREHZLEINN, J. Attuned Vibrations. [online] United Kingdom, 2016. [viewed 28 April 2017]. Available from: https://attunedvibrations.com/solfeggio-scale/\#

BRONZINO, J. D. The biomedical Engineering Handbook vol1. Boca Raton, FL: CRC Press, 1995.

BROWN, M. T. and BUSSELL, J. K. Medication Adherence: WHO Cares?. Mayo Clin Proc. 2011, 86(4), pp. 304-314.

CACACE, A. T. et al. Cutaneous-evoked tinnitus. II. Review of neuroanatomical, physiological and functional imaging studies. Audiol. Neurotol. 1999, vol. 4, pp. 258268.

CAIMMI, M. et al. Predicting Functional Recovery in Chronic Stroke Rehabilitation Using Event-Related Desynchronization-Synchronization during Robot-Assisted Movement. BioMed Research International. 2016, pp. 1-11.

CAMPBELL, D. El Efecto Mozart. Barcelona: Urano, 1998

CASTELLANOS, N. P. and MAKAROV, V. A. Recovering EEG brain signals: Artifact suppression with wavelet enhanced independent component analysis. Journal of Neuroscience Method. 2006, vol. 158, pp. 300-312.

CHAIEB, L., WILPERT, E. C., REBER, T. P. and FELL, J. Auditory beat stimulation and its effects on cognition and mood states. Frontiers in Psychiatry. 2015, 70(6).

CHOY, D. Method and apparatus for treatment of monofrequency tinnitus utilizing sound wave cancellation techniques. WO, Patent: 2004/017675 A1, 2004.

CHOY, D. Method and apparatus for treatment of predominant-tone and tinnitus. US, Patent: 2007/0093733 A1, 2007.

CIMA, R. F., CROMBEZ, G. and VLAEYEN, J. W. Catastrophizing and fear of tinnitus predict quality of life in patients with chronic tinnitus. Ear Hear. 2011, vol. 32, pp. 634641.

COHEN, M. X. Where does EEG come from? and What does it mean?. Trends in Neurosciences. 2017, 40(4), pp. 208-218.

COLLINS, B. T. The Importance of 432hz Music [online]. United Kingdom, 2017. [viewed 28 April 2017]. Available from: http://www.omega432.com/music.html 
CRESPO PELAyo, A. d. C., RECuero LÓPEZ, M., GÁlVEZ GARCíA, G. M. and BEGOÑA MONCO, A. Effect of binaural stimulation on attention and EEG. Archives of Acoustics. 2013, 38(4), pp. 517-528.

CURTIS, D. Binaural beats, brain wave entrainment and the hemi-sync process. The importance of the different brain wave states. Adelaide, South Australia: University of Adelaide, 2007.

DAVID, J. B., NAFTALI, A. and KATZ, A. Tinntrain: A multifactorial treatment for tinnitus using binaural beats. Hearing Journal. 2010, 63(11), pp. 25-28.

DAWES, P. et al. Hearing in middle age: a population snapshot of 40 - to 69 -year olds in the United Kingdom. Ear Hear. 2014, 35(3), pp. e44-51.

DE RIDDER, D. et al. An integrative model of auditory phantom perception: tinnitus as a unified percept of interacting separable subnetworks. Neurosci Biobehav Rev. 2014, vol. 44, pp. 16-32.

DELB, W. et al. Alterations in Event Related Potentials (ERP) associated with tinnitus distress and attention. Applied Psychophysiology and Biofeedback. 2008, 33(4), pp. 211-221.

DELORME, A. and MAKEIG, S. EEGLAB: an open source toolbox for analysis of singletrial EEG dynamics including independent component analysis. Journal of Neuroscience Methods. 2004, vol. 134, pp. 9-21.

DELORME, A. and MAKEIG, S. EEGLAB Wikitorial [online] USA, 2012. [viewed 21 August 2018]. Available from: ftp://sccn.ucsd.edu/pub/PDF EEGLAB Wiki Tutorial.pdf

DELORME, A., MIYAKOSHI, M., JUNG, T.-P. and MAKEIG, S. Grand average ERPimage plotting and statistics: A method for comparing variability in event-related singletrial EEG activities across subjects and conditions. J Neurosci Methods. 2015, vol. 250, pp. 3-6.

DEMARRE, D. A. and MICHAEL, D. Electroencephalographic measurements. In: Bioelectronic Measurements. New York: Prentice Hall, 1983, pp. 120-130.

DIESCH, E., ANDERMANN, M., FLOR, H. and RUPP, A. Functional and structural aspects of tinnitus-related enhancement and suppression of auditory cortex activity. Neurolmage. 2010, vol. 50, pp.. 1545-1559.

DRAGANOVA, R., ROSS, B., WOLLBRINK, A. and PANTEV, C. Cortical steady-state responses to central and peripheral auditory beats. Cerebral Cortex. 2008, vol. 18, pp. 1193-1200.

DREXLER, D. et. al. Systems and methods for a tinnitus therapy. US, Patent: US 9282917 B2, 2016.

DURRANT, J. D. and LOVRINIC, J. H. Bases of hearing science. Baltimore, Md: Williams and Wilkins, 1995. 
DYBALA, P. Hearing aid prices. [online]. United Kingdown 2016. [viewed 8 April 2017]. Available from: http://www.healthyhearing.com/help/hearingaids/prices

EGGERMONT, J. J. and ROBERTS, L. E. The neuroscience of tinnitus. Trends in Neurosciences. 2004, 27(11), pp. 676-682.

EGGERMONT, J. J. and TASS, P. A. Maladaptive neural synchrony in tinnitus: origin and restoration. Frontiers in Neurology. 2015, vol. 6, p. 29.

EICHHAMMER, P. et al. Neuronavigated repetitive transcranial magnetic stimulation in patients with tinnitus: a short case series.. Biol. Psychiatry. 2003, vol. 54, pp. 862-865.

ELGOYHEN, A. B., LANGGUTH, B., DERIDDER, D. and VANNESTE, S. Tinnitus: perspectives from human neuroimaging. Nature Reviews Neuroscience. 2015, vol. 16, pp. 632-642.

EL-SHUNNAR, S. K. et al. Primary care for tinnitus: practice and opinion among GPs in England. J Eval Clin Pract. 2011,17(4), pp. 684-92.

ENGLEHART, K. and HUDGINS, B. A robust, real-time control scheme for multifunction myoelectric control IEEE. Trans. Biomed. Eng. 2003, vol. 50, pp. 848-854.

ESPINOSA-SÁNCHEZ, J. M. Pharmacotherapy for tinnitus: Much ado about nothing. Rev Neurol. 2014, 59(4), pp. 164-174.

FIITZGIBBON, S. P., POWERS, D. M. W., POPE, K. J. and CLARK, C. R. Removal of EEG Noise an Artifact Using Blind Source Separation. Journal of Clinical Neurophysiology. 2007, 24(3).

FLOR, H., HOFFMANN, D., STRUVE, M. and DIESCH, E., 2004. Auditory discrimination training for the treatment of tinnitus. Applied Psychophysiology and Biofeedback, 29(2), pp. 113-120.

FORMBY, C. and SCHERER, R. Rationale for the tinnitus retraining therapy trial. Noise and Health. 2013, 15(63), p. 134.

FREESTONE, D. R., KAROLY, P. J. and COOK, M. J. A forward-looking review of seizure prediction. Current Opinion in Neurology. 2017, 30(2), pp. 167-173.

FRISCH, M. B. Quality-of-life-inventory. In: Encyclopedia of quality of life and well-being research, Dordrecht: Springer, 2014, pp. 5374-5377.

GABR, T. Q., EL-HAY, M. A. and BADAWY, A. Electrophysiological and psychological studies in tinnitus. Auris Nasus Larynx. 2011, vol. 33, pp. 678-683.

GALVEZ GARCIA, G. Desarrollo de tecnologías acústicas como terapia para la enfermedad de Parkinson. Madrid: E.T.S. DE INGENIEROS DE TELECOMUNICACION, 2015.

$\mathrm{GAO}, \mathrm{X}$. et al. Analysis of EEG activity in response to binaural beats with different frequencies. International Journal of Psychophysiology. 2014, 94(3), pp. 399-406. 
GARCIA-ARGIBAY, M. and SANTED, M. A. Efficacy of binaural auditory beats in cognition, anxiety, and pain perception: a meta-analysis. Psychological Research. 2019, 83(2), pp. 357-372.

GELLER, A. S. et al. Eye closure causes widespread low-frequency power increase and focal gamma attenuation in the human electrocorticogram. Clinical Neurophysiology. 2019, 125(9), pp. 1764-1773.

GERKEN, G. M., HESSE, P. S. and WIORKOWSKI, J. J. Auditory evoked responses in control subjects and in patients with problem-tinnitus. Hearing Research. 2001, vol. 157, pp. 52-64.

GHODRATITOOSTANI, I. et al. Theoretical Tinnitus Framework: A Neurofunctional Model. Front Neurosci.10. 2016, p. 370.

GIRAUD, A. L. et al. A selective imaging of tinnitus. Neuroreport. 1999, vol. 10, pp. 1-5.

GOLDMAN, J. Sonidos que sanan. Barcelona: Luciérnaga, 1992.

GOLDMAN, J. Los 7 Secretos de los Sonidos Sanadores (Tradición y sabiduría). Madrid: Gaia Ediciones, 2010.

GOLDSTEIN, B. A., LENHARDT, M. L. and SHULMAN, A. Tinnitus improvement with ultra-high-frequency vibration therapy. Int tinnitus J. 2005, vol. 11, pp. 14-22..

GOLDSTEIN, B. A. et al. Long-term inhibition of Tinnitus by UltraQuiet therapy. Preliminary report. Int tinnitus J. 2001, vol. 7, pp. 122-127.

GRAIMANN , B. and PFURTSCHELLE, G. Quantification and visualization of eventrelated changes in oscillatory brain activity in the time-frequency domain. Progress in Brain Research. 2006, vol.159, pp. 79-97.

GRAIMANN, B., HUGGINS, J. E., LEVINE, S. P. and PFURTSCHELLER,, G. Visualization of significant ERD/ERS patterns in multichannel EEG and ECoG data. Clinical Neurophysiology. 2002, 113(1), pp. 43-47.

GREIMEL, K. V., LEIBETSEDER, M., UNTERRAINER, J. and ALBEGGER, K. Can tinnitus be measured? Methods for assessment of tinnitus-specific disability and presentation of the Tinnitus Disability Questionnaire. HNO. 1999, vol. 47, pp. 196-201.

HAEFELI , M. and ELFERING, A. Pain assessment. Eur Spine J. 2006, 15(1), pp. S17S24.

HALL, D. et al. Systematic review of outcome domains and instruments used in clinical trials of tinnitus treatments in adults. Trials. 2016, vol. 17, pp. 1-19.

HANLEY, P. J., DAVIS, P. B., QUINN, S. A. and BEL, S. R. Treatment of tinnitus with a customized, dynamic acoustic neural stimulus: clinical outcomes in general private practice. Annals of Otology, Rhinology and Laryngology. 2008, 117(11), pp. 791-799.

HANSEN, C. and SNYDER, S. Active control of noise and vibration. Cambridge, UK: Cambridge University Press, 1997. 
HAUPTMANN, C. et. al. Acoustic coordinated reset neuromodulation in a real life patient population with chronic tonal tinnitus. Hindawi: BioMed Research International, 2015.

HAUPTMANN, C., WILLIAMS, M., VINCIATI, F. and HALLER, M. Technical Feasibility of Acoustic Coordinated Reset Therapy for Tinnitus Delivered via Hearing Aids: A Case Study. Hindawi: Case Reports in Otolaryngology, 2017. vol. 5304242.

HELLER, A. J. Classification and epidemiology of tinnitus. Otolaryngol Clin North Am. 2003, vol. 36, pp. 239-48.

HENRY, J. A. et al. Tinnitus Functional Index: Development, validation, outcomes research, and clinical application. Hearing Research. 2016, 334(1), pp. 58-64.

HENRY, J. A. et al. Tinnitus functional index: development, validation, outcomes research, and clinical application. Hear. Res. 2016. vol. 334, pp. 58-64.

HENRY, J. A., ZAUGG, T. L., MYERS, P. J. and SCHECHTER, M. A. Using therapeutic sound with progressive audiologic tinnitus management. Trends in Amplification. 2008, 12(3), pp. 188-209.

HERNÁDEZ-CORDERO, L. Respuestas evocadas auditivas. Asociación Española de Audiología [online]. Spain, 2015. [viewed 21 April 2017]. Available from: www.aedaweb.com.

HERRAIZ, C., DIGES, I. and COBO, P. Auditory discrimination therapy (ADT) for tinnitus management. Progress in Brain Research. 2007, vol. 166, pp. 467-471.

HERRAIZ, C. et al. Auditory discrimination training for tinnitus treatment: the effect of different paradigms. European Archives of Otorhinolaryngology. 2010, 267(7), pp. 10671074.

HERRAIZ, C., HERNANDEZ, F. J., TOLEDANO, A. and APARICIO, J. M. Tinnitus retraining therapy: prognosis factors. American Journal of Otolaryngology-Head and Neck Medicine and Surgery. 2007, vol. 28, pp. 225- 229.

HERRMANN, C. S., RACH, S., VOSSKUHL, J. and STRÜBER, D. Time-Frequency Analysis of Event-Related Potentials: A Brief Tutorial. Brain Topogr. 2014, vol. 27, pp. 438-450.

HOARE, D. J., GANDER, P. E., COLLINS, L. and SMITH, S. H. Management of tinnitus in English NHS audiology departments: An evaluation of current practice. $J$ Eval Clin Pract. 2012, vol.18, pp. 326-340.

HOFFMAN, H. J. and REED, G. W. Tinnitus: Theory and Management. In: Epidemiology of tinnitus. Shelton, CT: Pmph USA, 2004, pp. 16-42.

HONG, S. K., PARK, S., AHN, M. H. and MIN, B. K. Top-down and bottom-up neurodynamic evidence in patients with tinnitus. Hearing Research. 2016, vol. 342, pp. 86-100.

HOROWITZ, L. G. LOVE the Real da Vinci CODE. Sandpoint, ID: Tetrahedron Press, 2007. 
HOROWITZ, L. G. Musical Cult Control [online] United Kingdown, 2015. [viewed 28 April 2017]. Available from: http://medicalveritas.org/musical-cult-control/

HORSMAN, J., FURLONG, W., FEENY, D. and TORRANCE, G. The Health Utilities Index $(\mathrm{HUI})$ : concepts, measurement properties and applications. Health and quality of life outcomes. 2003, 1(1), p. 54.

HOUDAYER, E. et al.Involvement of cortico-subcortical circuits in normoacousic chronic tinnitus: A source localization EEG study. Clinical Neurophysiology. 2015, vol. 126, pp. 2356-2365.

HOWARD, D. M. and ANGUS, J. A. S. Acoustics and Psychoacoustics. UK: Focal Press, 2009.

HUANG, H. P. and CHEN, C. Y. Development of a myoelectric discrimination system for a multi-degree prosthetic hand. Proc. IEEE Int. Conf. on Robotics and Automation. 1999, vol. 3, pp. 2392-2397.

$\mathrm{HU}$, L. et al. Single-trial time-frequency analysis of electrocortical signals: Baseline correction and beyond. Neuroimage. 2014, vol. 84, pp. 876-887.

HUOTILAINEN, M. et al. International Conference on Auditory Display: Sleep Enhancement by Sound Stimulation. Atlanta, GA: Georgia Institute of Technology, 2015.

ITO, M., SOMA, K. and ANDO, R. Association between tinnitus retraining therapy and a tinnitus control instrument. Auris Nasus Larynx. 2009, vol. 36, pp. 536-540.

JASTREBOFF, P. J., GRAY, W. C. and GOLD, S. L., 1996. Neurophysiological Approach to Tinnitus Patients. American Journal of Otolog. 1996,17 (2).

JASTREBOFF, P. J. and JASTREBOFF, M. M. Tinnitus retraining therapy (TRT) as a method for treatment of tinnitus and hyperacusis patients. Journal American Academy of Audiology. 2000, 11(3), pp. 167-177.

JENKINS, J. S. The Mozart Effect. J R Soc Med. 2001, 94(4), pp. 170-172.

JIA, H., PENG, W. and HU, L. A novel approach to identify time-frequency oscillatory features in electrocortical signals. Journal of Neuroscience Methods. 2015, 253(10), pp. 18-27.

KADOURI, A., CORRUBLE, E. and FALISSARD, B. The improved Clinical Global Impression Scale (iCGI): development and validation in depression. BMC Psychiatry. 2007, 7(1), p. 7.

KALTENBACH, J. A. The dorsal cochlear nucleus as a participant in the auditory, attentional and emotional components of tinnitus. Hear Res. 2006, pp. 206, 200-226.

KAMALSKI, D. M. et al. Measuring disease-specific health-related quality of life to evaluate treatment outcomes in tinnitus patients: A systematic review. Otolaryngol Head Neck Surg August. 2010, vol. 143 no. 2, pp. 181-185. 
KAPPENMAN, E. S. and LUCK, S. J. ERP Components: The Ups and Downs of Brainwave Recordings. In: The Oxford Handbook of Event-Related Potential Components. China: Oxford University Press, 2012, pp. 1-30.

KARAKAS, S. A descriptive framework for information processing: an integrative approach. International Journal of Psychophysiology. 1997, 26(1), pp. 353-368.

KASPRZAK , C. Influence of Binaural Beats on EEG Signal. Acta Fhysica Polonica. 2011, vol. 119, pp. 986-990.

KENNEL, S., TAYLOR, A. G., LYON, D. and BOURGUIGNON, C. Pilot feasibility study of binaural auditory beats for reducing symptoms of inattention in children and adolescents with attention-deficit/hyperactivity disorder. J Pediatr Nurs. 2010, 25(1), pp. 3-11.

KERSTEN, P., WHITE, P. J. and TENNAN, A. Is the Pain Visual Analogue Scale Linear and Responsive to Change? An Exploration Using Rasch Analysis. PLoS One. 2014, 9(6), p. e99485.

KHORSHIDTALAB, A., SALAMI, M. J. E. and HAMEDI, M. Robust classification of motor imagery EEG signals using statistical time-domain features. Physiol. Meas. 2013, vol. 34, pp. 1563-1579.

KIM, B. J., CHUNG, S. W., JUNG, J. Y. and SUN, M. W. Effect of different sounds on the treatment outcome of tinnitus retraining therapy. Clinical and experimental otorhinolaryngology. 2014, 7(2), p. 87.

KLIMESCH, W. EEG alpha and theta oscillations reflect cognitive and memory performance: a review and analysis. Brain research reviews. 1999, 29(2), pp. 169-195.

KLIMESCH, W.. An algorithm for the EEG frequency architecture of consciousness and brain body coupling. Frontiers in Human Neuroscience. 2013, vol. 7, pp. 1-4.

KOCHKIN, S., TYLER, R. and BORN, J. MarkeTrak VIII: The Prevalence of Tinnitus in the United States and the Self-reported Efficacy of Various Treatments Hearing Review. Hearing review. 2011, 18(12), pp. 10-27.

KOELSCH, S. A neuroscientific perspective on music therapy. Annals of the New York Academy of Sciences. 2009, pp. 1169, 374-384.

KRAL, A. and O'DONOGHUE, G. M. Profound deafness in childhood. $N$ Engl $J$ Med. 2010, 363(15), pp. 1438-50.

KRAUSE, C. Brain electric oscillations and cognitive processes. In: Experimental Methods in Neuropsychology. Amsterdam: Springer, 2003, pp. 111-130.

KRAUSE, C. M. Event-related Desynchronization (ERD) and Synchronization (ERS) During Auditory Information Processing. Journal of New Music Research. 1999, 28(3), pp. 257-265.

KRAUSE, C. M. et al. Event-related desynchronization evoked by auditory stimuli. Brain Topography. 1994, 7(2), pp. 107-112. 
KRAUS, J. and PORUBANOVÁ, M. The Effect of Binaural Beats on Working Memory Capacity. Studia Psychologica. 2015, 38 (2), pp. 135-145.

KROL, L. R. et al. SEREEGA: Simulating Event-Related EEG Activity. J Neurosci Methods. 2018, vol.309, pp.13-24.

KROPOTOV, J. D. Quantitative EEG Event-Related Potentials and Neurotherapy. San Diego, California: Academic Press - Elsevier, 2009.

LAHIRI, N. and DUNCAN, J. S. The Mozart effect: encore. Epilepsy Behav. 2007, 11(1), pp. 152-153.

LANDGREBE, $M$. et al. The Tinnitus Research Initiative (TRI) database: a new approach for delineation of tinnitus subtypes and generation of predictors for treatment outcome. BMC Med. Inform. Decis. Mak. 2010, vol. 10, p. 42.

LANE, J. D., KASIAN, S. J., OWENS, J. E. and MARSH, G. R. Binaural auditory beats affect vigilance performance and mood. Physiol Behav. 1998, 63(2), pp. 249-252.

LANGGUTH, B. et al. The impact of auditory cortex activity on characterizing and treating patients with chronic tinnitus - first results from a PET study. Acta Otolaryngol. Suppl. 2006, p. 84-88.

LANGGUTH, B. et al. Consensus for tinnitus patient assessment and treatment outcome measurement: Tinnitus Research Initiative meeting, Regensburg, July 2006. Prog. Brain Res. 2007, vol. 166, pp. 525-536.

LANGGUTH, B., KREUZER, P. M., KLEINJUNG, T. and DE RIDDER, D. Tinnitus: causes and clinical management. Lancet Neurol. 2013, vol. 12, pp. 920-930.

LANTING, C. P., DE KLEINE, E., BARTELS, H. and VAN DIJK, P. Functional imaging of unilateral tinnitus using fMRI. Acta Otolaryngol. 2008, vol. 128, pp. 415-421.

LE SCOUARNEC, R.-P., POIRIER, R.-M., OWENS, J. E. and GAUTHIER, J. Use of binaural beat tapes for treatment of anxiety: a pilot study of tape preference and outcomes. Altern Ther Health Med. 2001, 7(1), pp. 58-63.

LEE , K., MAKINO , K. and YAMAHARA, K. Evaluation of tinnitus retraining therapy for patients with normal audiograms versus patients with hearing loss. Auris Nasus Larynx. 2017, 45 (2), pp.215-221.

LEE, C.-H.et al. Auditory Cortical Evoked Potentials in Tinnitus Patients with Normal Audiological Presentation. J Formos Med Assoc. 2007, 106(12), pp. 979-985.

LEE, K., MAKINO, K. and YAMAHARA, K. Evaluation of tinnitus retraining therapy for patients with normal audiograms versus patients with hearing loss. Auris Nasus Larynx. 2018, , 45(2), pp. 215-221.

$\mathrm{LEI}, \mathrm{Y}$. Intelligent fault diagnosis and remaining useful life prediction of rotating machinery. Cambridge, MA: Xi'an Jiaotong University Press Co. Published by Elsevier Inc, 2017. 
LENHARDT, M. L. Tinnitus Devices. In: Encyclopedia of Biomedical Engineering and Biomaterials. Boca de Raton, Fl: Medical College of Virginia, Virginia Commonwealth University, 2004.

LEVENSTEIN, S. et al. Development of the perceived stress questionnaire: a new tool for psychosomatic research. J. Psychosom. Res. 1993, vol. 37, pp. 19-32.

$\mathrm{Li}, \mathrm{L}$. The differences among eyes-closed, eyes-open and attention states: an EEG study. IEEE 6th International Conference on Wireless Communications Networking and Mobile Computing (WiCOM). Chengdu, China, 2010.

LINDBERG, P., SCOTT, B., MELIN, L. and LYTTKENS, L. Long-Term Effects of Psychological Treatment of Tinnitus. Scandinavian Audiology. 1987, 16(3), pp. 167-172.

LITHFOUS, S., DUFOUR, A., BLANC, F. and DESPRÉS, O. Allocentric but Not Egocentric Orientation Is Impaired During Normal Aging: An ERP Study. Neuropsychology. American Psychological Association. 2014, 28(5), pp.761-771

LOCKWOOD, A. H. et al. The functional neuroanatomy of tinnitus: evidence for limbic system links and neural plasticity. Neurology. 1998 , vol. 50, pp. 114-120.

LONDERO, A. and HALL, D. A. Call for an Evidence-Based Consensus on Outcome Reporting in Tinnitus Intervention Studies. Front. Med. 2017, 4(42).

LOPEZ-CALDERON, J. and LUCK, S. J. ERPLAB: an open-source toolbox for the analysis of event-related potentials. Front Hum Neurosci. 2014, 8(213).

LUCK, S. J. Event-related potentials: A methods handbook. New York: MIT Press, 2005.

MAES, I. H. et al. Assessment of health state in patients with tinnitus: a comparison of the EQ-5D and HUI mark III. Ear Hear. 2011, vol. 32, pp. 428-435.

MAES, I. H. L. et al. Tinnitus: A Cost Study. Ear and Hearing. 2013, vol. 34, pp. 508514.

MALYKH, A. G. and SADAIE, M. R. Piracetam and piracetam-like drugs: from basic science to novel clinical applications to CNS disorders. Drugs, 2010, 70(3), pp. 287-312.

MARTINEZ, C., WALLENHORST, C., MCFERRAN, D. and HALL, D. A. Incidence Rates of Clinically Significant Tinnitus: 10-Year Trend From a Cohort Study in England. Ear Hear. 2015, 36(3), p. e69-e75.

MCPHERSON, B. Innovative technology in hearing instruments: matching needs in the developing world. Trends Amplif. 2011, 15(4), pp. 209-14.

MEIKLE, M. B. et al. The tinnitus functional index: development of a new clinical measure for chronic, intrusive tinnitus. Ear Hear. 2012, vol. 33, pp. 153-176.

MEIKLE, M. B., STEWART, B. J. and GRIEST, S. E. Tinnitus outcomes assessment. Trends Amplif. 2008, vol. 12, pp. 223-235.

MELCHER, J. R., SIGALOVSKY, I. S., GUINAN Jr, J. J. and LEVINE, R. A. Lateralized tinnitus studied with functional magnetic resonance imaging: abnormal inferior colliculus activation. J. Neurophysiol. 2000, vol. 83, pp. 1058-1072. 
MELIN, L., SCOTT, B., LINDBERG , P. and LY, L. Hearing aids and tinnitus -an experimental group study. British Journal of Audiology. 1987, 21(2), pp. 91-97.

MEYER, M. et al., 2014. Disentangling Tinnitus Distress and Tinnitus Presence by Means of EEG Power Analysis. Neural Plasticity. 2014, vol. 468546, pp. 1-13.

MINDLABPRO. Nootropics for Tinnitus - Balance Brain Chemicals for Healthy Hearing [online]. United Kingdom, 2018. [viewed 23 December 2018]. Available from: https://www.mindlabpro.com/blog/nootropics/nootropics-tinnitus

MIRZ, F., GJEDDE, A., SODKILDE-JORGENSEN, H. and PEDERSEN, C. B. Functional brain imaging of tinnitus-like perception induced by aversive auditory stimuli. Neurore- port. 2000, vol. 11, pp. 633-637.

MIRZ, F. et al. Position emission tomography of cortical centers of tinnitus. Hear Res. 1999, vol. 134, p. 133-144.

MISKOVIC, V., MACDONALD, K. J., RHODES, L. J. and COTE, K. A. Changes in EEG multiscale entropy and power-law frequency scaling during the human sleep cycle. Wiley Online Library - Human Brain Mapping. 2018, 40(2).

MOHR, P. E. et al. The societal costs of severe to profound hearing loss in the United States. Int J Technol Assess Health Care. 2000, 6(4), pp. 1120-35.

MUHLAU, M. et al. Structural brain changes in tinnitus. Cereb. Cortex. 2006 , vol. 16, pp. 1283-1288.

MÜLLER, K. et al. Validation of Online Versions of Tinnitus Questionnaires Translated into Swedish. Front. Aging Neurosci. 2016, 8(272).

NEFF, $P$. et al. Active listening to tinnitus and its relation to resting state EEG activity. Neuroscience Letters. 2019, vol. 694, pp. 176-183.

NEFF, P. et al. $10 \mathrm{~Hz}$ Amplitude Modulated Sounds Induce Short-Term Tinnitus Suppression. Front. Aging Neurosci. 2017, 9(130).

NEWMAN, C. W., JACOBSON, G. P. and SPITZER, J. B. Development of the Tinnitus Handicap Inventory. Arch. Otolaryngol. Head Neck Surg. 1996, vol. 122, pp. 143-148.

NEWMAN, C. W., SANDRIDGE, S. A. and JACOBSON, G. P. Psychometric adequacy of the Tinnitus Handicap Inventory (THI) for evaluating treatment outcome. J. Am. Acad. Audiol. 1998, vol. 9, pp. 153-160.

NHS. National Health Service. Cost of missed appointment in 2014. [online]. United Kingdown, 2014. [viewed 8 April 2017]. Available from: https://www.england.nhs.uk/2014/03/missed-appts/

NIEDERMEYER; F., LOPES-DA-SILVA, F. Neurocognitive functions and the EEG. In: Electroencephalography: Basic Principles, Clinical Applications and Related Fields. Philadelphia, Lippincott: Williams and Wilkins, 2005, pp. 661-683.

NOBLE, W. and TYLER, R. Physiology and phenomenology of tinnitus: implications for treatment. Int J Audiol. 2007, pp. 46(10), pp. 569-575. 
NONDAHL, D. M. et al. The ten-year incidence of tinnitus among older adults. Int $J$ Audiol. 2010, 49(8), pp. 580-585.

NOREÑA, A. J. and EGGERMONT, J. J. Enriched acoustic environment after noise trauma reduces hearing loss and prevents cortical map reorganization. The Journal of Neuroscience. 2005, 25(3), pp. 699-705.

NORHAZMAN, $\mathrm{H}$. et al. The investigation of alpha frontal energy asymmetry on normal and stress subjects after listening to the binaural beats $10 \mathrm{~Hz}$. IEEE 10th International Colloquium on Signal Processing and its Applications. Kuala Lumpur, Malaysia, 2014.

OLUSANYA, B. O., NEUMANN, K. J. and SAUNDERS, J. E. The global burden of disabling hearing impairment: a call to action. Bull World Health Organ. 2014, 92(5), pp. 367-373.

ORTIZ, T. et al. Impact of auditory stimulation at a frequency of $5 \mathrm{~Hz}$ in verbal memory. Actas Esp Psiquiatr. 2007,, 36(6), pp. 307-313.

OSKOEI, M. A. and HU, H. GA-based feature subset selection for myoelectric classification. ROBIO'06: IEEE Int.Conf. on Robotics and Biomimetics. 2006, pp. 14651470.

OSTER, G. Auditory beats in the brain. Sci Am. 1973, 229(4), pp. 94-102.

PADMANABHAN, R., HILDRETH, A. J. and LAWS, D. A prospective, randomised, controlled study examining binaural beat audio and pre-operative anxiety in patients undergoing general anaesthesia for day case surgery. Anaesthesia. 2005, 60(9), pp. 874-877.

PÁEZ, M. Tinitus: avances en fisiopatología y tratamiento. Arch Neurocien (Mex). 2006, 11(2), pp. 112-122.

PAPE, J. et al. Playing and listening to tailor-made notched music: cortical plasticity induced by unimodal and multimodal training in tinnitus patients. Hindawi: Neural Plasticity. 2014, vol. 2014, article ID 516163

PARAZZINI, M. et al. Open ear hearing aids in tinnitus therapy: An efficacy comparison with sound generators. International Journal of Audiology. 2011, 50(8), pp. 548-553.

PAUL, B. T. et al. Modulation of Electrocortical Brain Activity by Attention in Individuals with and without Tinnitus. Neural Plasticity. 2014, vol. 1, p. 16.

PEÑARANDA, A. et al. Economic benefits of the cochlear implant for treating profound sensorineural hearing loss. Rev Panam Salud Publica. 2012,, 31(4), pp. 325-331.

PENG, W. and TANG, D. Pain Related Cortical Oscillations: Methodological Advances and Potential Applications. Frontiers in Computational Neurosciences. 2016, 10(9), pp. 1-10.

PÉREZ-GONZÁLEZ, D. and MALMIERCA, M. . S. Adaptation in the auditory system: an overview. Frontiers in Integrative Neuroscience. 2014, 8(19), pp. 1-10.

PETROVICH, O. The Effects of Binaural Beats on Emotion and Cognition. St. Petersburg, FI: University of South Florida St. Petersburg, 2018. 
PFURTSCHELLER, G. and LOPES DA SILVA, F. H. Event-related EEG/MEG synchronization and desynchronization: basic principles. Clinical Neurophysiology. 1999, 110(1), pp. 1842-1857.

PHINYOMARK, A., LIMSAKUL, C. and PHUKPATTARANONT, P. A novel feature extraction for robust EMG pattern recognition. 2009, Issue arXiv:0912.3973.

PICTON, T. W., HILLYARD, S. A., KRAUSZ, H. I. and GALAMBOS, R. Human auditory evoked potentials. I. Evaluation of components.. Electroencephalogr. Clin. Neurophysiol. 1974, vol. 36, pp. 179-190.

PICTON, T. W. and FITZGERALD, P. G. A general description of the human auditory evoked potentials. In: Bases of Auditory Brain-Stem Evoked Responses. New York: Grune and Stratton, 1983.

PIERZYCKI, R. H., MCNAMARA, A. J., HOARE, D. J. and HALL, D. A. Whole scalp resting state EEG of oscillatory brain activity shows no parametric relationship with psychoacoustic and psychosocial assessment of tinnitus: A repeated measures study. Hearing Research. 2016, vol. 331, pp. 101-108.

PIVIK, R. T. et al. Guidelines for the recording and quantitative analysis of electroencephalographic activity in research contexts. Psychophysiology. 1993, 30(6), pp. 547-558.

POSTHUMA, D., NEALE, M. C., BOOMSMA, D. I. and DE GEUS, E. J. Are smarter brains running faster? Heritability of alpha peak frequency, $I Q$, and their interrelation. Behavior Genetics. 2001, 31(6), pp. 567-79.

POWERS, L. and DOS SANTOS, G. M. Acoustic theraphy for the treatment of tinnitus: A premier. Hearing Review. 2015, Tech Topic(Tinnitus), pp. 22-24.

PROVERBIO, A. M. and ZANI, A. Electromagnetic manifestations of mind and brain. Elsevier Science, Academic Press. 2002, vol. The Cogntive Electrophysiology of Mind and Brain, pp. 13-40.

PSSRU. Personal Social Services Research Unit. Unit Costs of Health and Social Care 2013 [online]. United Kingdown, 2013. [viewed 8 April 2017]. Available from: http://www.pssru.ac.uk/project-pages/unit-costs/2013/

PULLON, R. M., MCCABE, S. and GASKELL, A. Non-sinusoidal waves in the EEG and their simulated effect on anaesthetic quantitative EEG monitors. Springer Netherlands Jornal of Clinical Monitoring and Computing, 2019, pp. 1-8.

RAHMAN, A. et al. EEG based Brain Alertness Monitoring by Statistical and Artificial Neural Network Approach. International Journal of Advanced Computer Science and Applications. 2019, 10(1), pp. 431-442.

RATCLIFFE, C. P. and RATCLIFFE, B. Type A and Type B Elemental Uncertainties. In: Doubt-Free Uncertainty In Measurement. An Introduction for Engineers and Students. Switzerland: Springer International Publishing, 2015.

RAUSCHECKER, J. P., LEAVER, A. M. and MÜHLAU, M. Tuning out the noise: Limbicauditory interactions in tinnitus. Neuron 66(6). 2010, p. 819-826. 
RAUSCHER, F. H., SHAW, G. L. and KY, C. N. Music and spatial task performance. Nature. 1993,, vol. 365, p. 611.

RAVIGNANI, A., SONNWEBER, R.-S., STOBBE, N. and FITCH, W. T. Action at a distance: dependency sensitivity in a New World primate. Biology Letters. 2013, 9(3).

RAVISHANKAR, S. N. A comparative study to determine the efficacy of Piracetam over Carbamazepine in the treatment of Idiopathic Tinnitus. International Journal of Innovative Research in Medical Science. 2017, 2(7).

REAVIS, K. M. et al. Temporary suppression of tinnitus by modulated sounds. Journal of the Association for Research in Otolaryngology. 2012, 13(4), pp. 561-571.

RECHY-RAMIREZ, E. J. and HU, H. Stages for developing control systems using EMG and EEG signals: a survey Technical Report CES-513. Essex, UK: School of Computer Science and Electronic Engineering, University of Essex, 2011.

REEDIJK, S. A., BOLDERS, A. and HOMMEL, B. The impact of binaural beats on creativity. Front Hum Neurosci. 2013, vol. 7, p. 786.

REEDIJK, S., BOLDERS, A., COLZATO, L. and HOMMEL, B. Eliminating the attentional blink through binaural beats: a case for tailored cognitive enhancement. Frontiers in Psychiatry. 2015, 6(82).

REINHARDT, U. and LANGE, E. Effect of music on depressed patients. Psychiatr Neurol Med Psychol. 1982, 34(7), pp. 414-21.

REYES, S. A. et al. Brain imaging of the effects of lidocaine on tinnitus. Hear Res. 2002, vol. 171, pp. 43-50.

RICHARDSON, S. J., BROOKS, H. L., BRAMLEY, G. and COLEMAN, J. J. Evaluating the Effectiveness of Self-Administration of Medication (SAM) Schemes in the Hospital Setting: A Systematic Review of the Literature. PLoS ONE. 2014, 9(12), pp. 1-18.

ROBERTS, L. E., BOSNYAK, D. J. and THOMPSON, D. C. Neural plasticity expressed in central auditory structures with and without tinnitus. Frontiers in Systems Neuroscience. 2012, 6(40), pp. 1-17.

ROBERTS, L. E., MOFFAT, G. and BOSNYAK, D. J. Residual inhibition functions in relation to tinnitus spectra and auditory threshold shift. Acta Oto-Laryngologica. 2006, 126(S556), pp. 27-33.

RODRIGO, M. S. Musicoterapia. Terapia de música y sonido. Madrid: Musicalis, 2008.

SALTZMAN, M. and ERSNER, M. S. A hearing aid for relief of tinnitus aurium. Laryngoscope. 1947, pp. 57, 358-366.

SAUNDERS, J. and BARRS, D. Cochlear implantation in developing countries as humanitarian service: physician attitudes and recommendations for best practice. Otolaryngol Head Neck Surg. 2011, 145(1), pp. 74-9.

SCHABUS, M., 2001. Cognitive Electrophysiology and Attention: Early evoked EEG components, attention and brain oscillations. Institute of Psychology, University of Salzburg. 
SCHAETTE, R. and KEMPTER, R. Development of tinnitus-related neuronal hyperactivity through homeostatic plasticity after hearing loss: a computational model. Eur J Neurosci. 2006, vol. 23, pp. 3124-3138.

SCHECKLMANN, M., LANDGREBE, M., LANGGUTH, B. and TRI Database Study Group. Phenotypic characteristics of hyperacusis in tinnitus. PLoS ONE. 2014, vol. 9, p. e86944.

SEDLEY, W. et al. An Integrative Tinnitus Model Based on Sensory Precision. Trends in Neurosciences. 2016, 39(12), pp. 799 - 812.

SHORE, S. E., ROBERTS, L. E. and LANGGUTH, B. Maladaptive plasticity in tinnitus - triggers, mechanisms and treatment. Nature Reviews Neurology. 2016, vol. 12, pp. 150-160.

SI, L., WANG , Z., TAN , C. and LIU, X. Vibration-Based Signal Analysis for Shearer Cutting Status Recognition Based on Local Mean Decomposition and Fuzzy C-Means Clustering. Appl. Sci. 2017, 7(164).

SISMANIS, A. Pulsatile tinnitus: a 15-year experience. Am J Otol. 1998, vol. 19, pp. 472-477.

SISMANIS, A. Tinnitus: advances in diagnosis and management. Alexandria, VA: Amer Academy of Otolaryngology, 1999.

SMITH, J. M. Chakra Balancing Music and Sound Healing for Your Well Being. [online] United Kingdown, 2017. [viewed 28 April 2017]. Available from: http://balance.chakrahealingsounds.com/1st-chakra/

SMITS, M. et al. Lateralization of functional magnetic resonance imaging (fMRI) activation in the auditory pathway of patients with lateralized tinnitus. Neuroradiology. 2007, vol. 49, pp. 669-679.

SOLCÀ, M., MOTTAZ, A. and GUGGISBERG, A. G. Binaural beats increase interhemispheric alpha-band coherence between auditory cortices. Hearing Research. 2016, vol. 332, pp. 233-237.

SÖRNMO, L. and LAGUNA, P. Bioelectrical Processing in Cardiac and Neurological Applications. New York: Academic Press, 2005.

SPIEGEL, D. P. et al. Multisensory attention training for treatment of tinnitus. Scientific Reports. 2015, 28(5), p. 10802.

STEIN, A. et al.Clinical trial on tonal tinnitus with tailor-made notched music training. BMC Neurology. 2016, 16(1), p. 38.

STOICA, P. and MOSES, R. Spectral Analysis of Signals. New Jersey: Prentice Hall, 2005.

STOUFFER, J. L. and TYLER, R. S. Characterization of tinnitus by tinnitus patients. $J$ Speech Hear Disord,. 1990, 55(3), pp. 439-453. 
STOUGH, C., DONALDSON, C., SCARLATA, B. and CIORCIARI, J. Psychophysiological correlates of the NEO PI-R openness, agreeableness and conscientiousness: preliminary results. Int J Psychophysiol. 2001, 41(1), pp. 87-91.

SURR, R. K., MONTGOMERY, A. A. and MUELLER, H. Effect of Amplification on Tinnitus Among New Hearing Aid Users. Ear and Hearing. 1985, 6(2).

SURR, R. K., WALDEN, B. E., CORD, M. T. and OLSON, L. Influence of environmental factors on hearing-aid microphone preference. J Am Acad Audiol. 2002, 13(6), pp. 308322.

SWEETOW, R. The use of fractal tones in tinnitus patient management. Noise and Health. 2013, March-April, vol. 15.

SWEETOW, R. W. and SABES, J. H. Effects of acoustical stimuli delivered through hearing aids on tinnitus. J Am Acad Audiol. 2010, vol. 21, pp. 461-73.

TAVIRA SANCHEZ, F. J. Propuesta de Metodología de terapia acústica en Parkinson Diploma de Estudios Avanzados. Madrid: ETSII, Universidad Politécnica Madrid, 2010.

TELLES, S., DEEPESHWAR, S., NAVEEN, K. and PAILOOR, S. Long Latency Auditory Evoked Potentials during Meditation. Clinical EEG and Neuroscience. 2015, 46(4), pp. 299-309.

TELLES, S. et al. P300 and Heart Rate Variability Recorded Simultaneously in Meditation. Clinical EEG and Neuroscience. 2018, 50(3), pp. 161-171

TELLES, S. et al. A fMRI Study of Stages of Yoga Meditation Described in Traditional Texts. Journal of Psychology and Psychotherapy. 2014, 5(3).

TOMATIS, A. Tomatis: History and Development. [online] France, 2017. [viewed 10 April 2017]. Available from: http://www.tomatis.com/en/thegroup/history-and-development.html

USELTON, J. P., KIENLE, P. C. and MURDAUGH, L. B. Prevention of SelfAdministration Error. In: Assuring Continuous Compliance with Joint Commission Standards: A Pharmacy Guide. Bethesda, MD: American Society of Health-System Pharmacists, 2010.

VAN DE HEYNING, P. et al. Efficacy and safety of AM-101 in the treatment of acute inner ear tinnitus-a double-blind, randomized, placebo-controlled phase II study. Otology and Neurotology. 2014, 35(4), p. 589.

VANNESTE, S. et al. Neuronal correlates of maladaptive coping: an EEG-study in tinnitus patients. Plos One. 2014, 9(2), p. e88253.

VANNESTE, S. et al. Does enriched acoustic environment in humans abolish chronic tinnitus clinically and electrophysiologically? A double blind placebo controlled study. Hearing Research. 2013, vol. 296(1), pp. 141-148.

VERNON, D., PERYER, G., LOUCH, J. and SHAW, M. Tracking EEG changes in response to alpha and beta binaural beats. International Journal of Psychophysiology. 2014, 93(1), pp. 134-139. 
WAHBEH, H., CALABRESE, C. and ZWICKEY, H. Binaural beat technology in humans: a pilot study to assess psychologic and physiologic effects. J Altern Complement Med, 2007, 13(1), pp. 25-32.

WAHBEH, H., CALABRESE, C., ZWICKEY, H. and ZAJDEL, D. Binaural beat technology in humans: a pilot study to assess neuropsychologic, physiologic, and electroencephalographic effects. J Altern Complement Med. 2007, 13(2), pp. 199-120.

WALPURGER, V., HEBING-LENNARTZ, G., DENECKE, H. and PIETROWSKY, R. Habituation deficit in auditory event-related potentials in tinnitus complainers. Hearing Research. 2003, 181(1), pp. 57-64.

WANG, $H$. et al. Regional glucose metabolic increases in left auditory cortex in tinnitus patients: a preliminary study with positron emission tomography. Chin. Med. J. 2001, vol. 114, pp. 848-851.

WARD, C. and OBEID, I. Application of identity vectors for EEG classification. Elsevier ScienceDirect - Journal of Neuroscience Methods. 2019, vol. 311, pp. 338-350.

WEILAND, T. J. et al. Original sound compositions reduce anxiety in emergency department patients: a randomised controlled trial. Med J Aust. 2011, 195(11-12), pp. 694-698.

WEISE, C. et al.Acceptance of tinnitus: validation of the tinnitus acceptance questionnaire. Cognitive Behaviour Therapy. 2013, 42(2), pp. 100-115.

WEISZ, N., VOSS, S., BERG, P. and ELBERT, T. Abnormal auditory mismatch response in tinnitus sufferers with high-frequency hearing loss is associated with subjective distress level. BMC Neurosciences. 2004, 5(1), p. 8.

WESTIN, V. Z. et al. Acceptance and commitment therapy versus tinnitus retraining therapy in the treatment of tinnitus: a randomised controlled trial. Behaviour Research and Therapy. 2011, 49(11), pp. 737-747.

WHO. Development of the World Health Organization WHOQOL-BREF quality of life assessment. The WHOQOL Group. Psychol. Med. 1998, vol. 28, pp. 551-558.

WHO. WHO global estimates on prevalence of hearing loss. [online] Switzerland 2012. [viewed 8 April 2017]. Available from: http://www.who.int/pbd/deafness/estimates/en/

WILLIAMS, M., HAUPTMANN, C. and PATEL, N. Acoustic CR neuromodulation therapy for subjective tonal tinnitus: a review of clinical outcomes in an independent audiology practice setting. Front. Neurol. 2015, 6(54).

WILSON, P. H., HENRY, J., BOWEN, M. and HARALAMBOUS, G. Tinnitus reaction questionnaire: psychometric properties of a measure of distress associated with tinnitus. Journal of Speech, Language, and Hearing Research. 1991, 34(1), pp. 197-201.

YOUNG, C. W.et al. Investigate the effect of EEG for relaxation using binaural beats. 7th International Symposium on Machinery and Mechatronics for Agriculture and Biosystems Engineering (ISMAB), Yilan, Taiwan, 2014. 
ZAVALA-YOE, R. and RAMIREZ-MENDOZA, R. A. Dynamic complexity measures and entropy paths for modelling and comparison of evolution of patients with drug resistant epileptic encephalopathy syndromes (DREES). Metabolic Brain Disease. 2017, pp. 117.

ZEMAN, F. et al. Which tinnitus-related aspects are relevant for quality of life and depression: results from a large international multicentre sample. Health Qual. Life Outcomes. 2014, 12(7).

ZENG, F.-G.et al. Tinnitus Suppression by Low-Rate Electric Stimulation and Its Electrophysiological Mechanisms. Hear Res. 2011, 277(1-2), pp. 61-66.

ZIGMOND, A. S. and Snaith, R. P. The hospital anxiety and depression scale. Acta Psychiatrica Scandinavica. 1983, vol. 67, pp. 361-370. 
INDEXES 


\section{FIGURES}

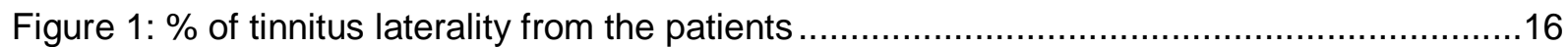

Figure 2: $\%$ of patients with different tinnitus frequency tones ..........................................16

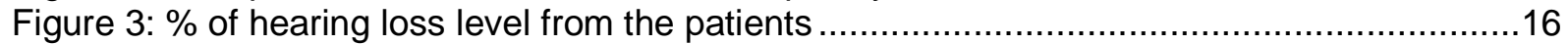

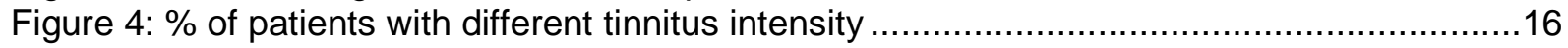

Figure 5: Available laboratory at ITESM, where the measurements were taken ......................19

Figure 6: (a) the equipment employed to record the EEG signals; (b) the 17 EEG channels based on the 10/20 system which were used. Only channels P3 and P4 could not be recorded

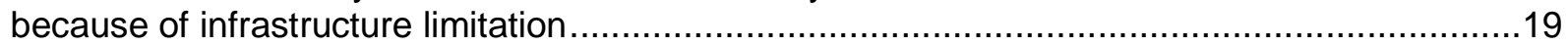

Figure 7: Study design of the protocol. Patients monitored for 8 weeks .................................25

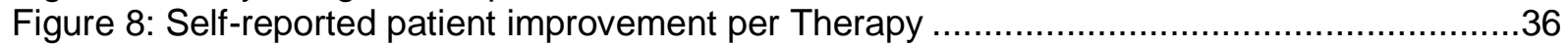

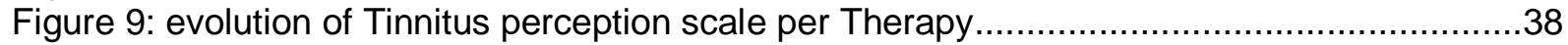

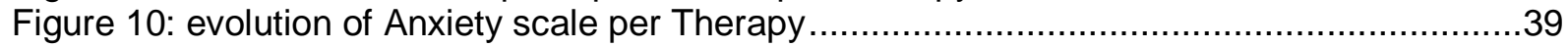

Figure 11: evolution of Depression scale per Therapy......................................................... 40

Figure 12: Mean and standard deviation process calculation per therapy from the difference of

the time and frequency domain parameters of the patients ...............................................42

Figure 13: EO analysis: Frontal and temporal lobe behavior per therapy shown by the difference

between session 4 and session 1 of the Mean (a) and MNF (b) representation .......................45

Figure 14: Subjective analysis summary per therapy after two-month treatment in line with

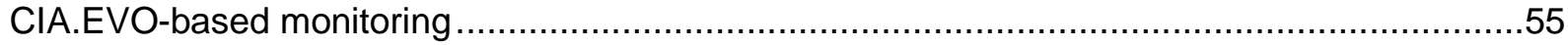

Figure 15: Tinnitus diagnostic and therapeutic steps versus specific conditions .....................107

Figure 16: Age and sex-specific trends in chronic tinnitus from the U.S. National Health

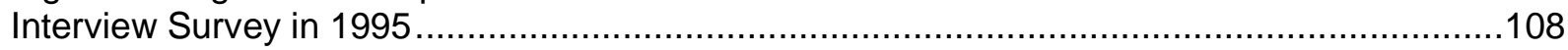

Figure 17: Sample of TRT signal, time and frequency analysis ........................................111

Figure 18: Sample of TEAE signal: time and frequency analysis.......................................111

Figure 19: Sample of Binaural signal: time and frequency analysis ...................................111

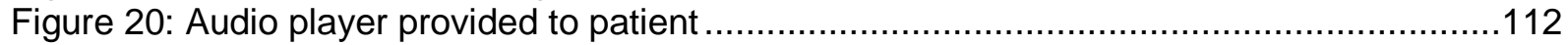

Figure 21: Assembly to evaluate the audios connecting (a) the audio player directly with the

Analyser and (b) through Headphones and SPL Meter ..................................................112

Figure 22: Frequency response of the audio player playing a pure tone at $232 \mathrm{~Hz}$ connecting (a)

it directly with the Analyser and (b) through Headphones and SPL Meter ...........................112

Figure 23: Diagrammatic representation of the auditory evoked potential components ............115

Figure 24: online questionnaires answered by patients through Google forms ......................124

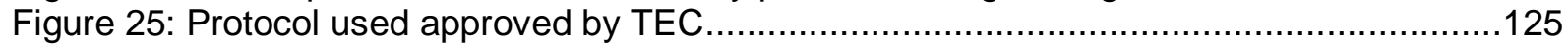

Figure 26: Subjective patient score representation.......................................................127

Figure 27: evolution of Tinnitus perception scale per Therapy at initial CIA.EVO 2 and 3.......128

Figure 28: EC analysis: Frontal and temporal lobe behavior per therapy shown by the difference

between session 4 and session 1 of the Mean (a) and MNF (b) representation .....................144

Figure 29: Acoustic therapy condition analysis: Frontal and temporal lobe behavior per therapy

shown by the difference between sessions 4 and 1 of the Mean (a) and MNF (b) ..................153

Figure 30: GAv ERP of 16 channels from all patients using Binaural $f_{0}$ Session 1 ..................160

Figure 31: GAv ERP of 16 channels from all patients using Binaural $f_{0}$ Session $4 \ldots \ldots \ldots \ldots \ldots \ldots . . . .161$

Figure 32: GAv ERP of 16 channels from all patients using Binaural $396 \mathrm{~Hz}$ Session 1 ..........162

Figure 33: GAv ERP of 16 channels from all patients using Binaural 396Hz Session $4 \ldots \ldots \ldots . .163$

Figure 34: GAv ERP of 16 channels from all patients using TRT Session 1 ..........................164

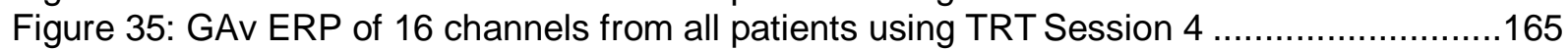

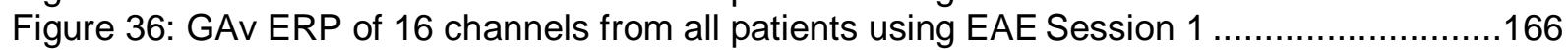

Figure 37: GAv ERP of 16 channels from all patients using EAE Session 4 .......................167 
Figure 38: ERPs Principal and Independent Component Analysis for Patient 3 - Binaural $f_{0}$ (Session 1 and 4).

Figure 39: ERPs Principal and Independent Component Analysis for Patient 9 - Binaural 396 Hz

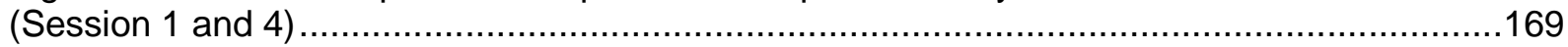

Figure 40: ERPs Principal and Independent Component Analysis for Patient 5 - TRT (Session 1

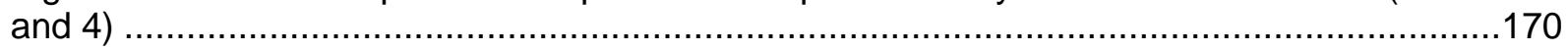

Figure 41: ERPs Principal and Independent Component Analysis for Patient 7 - EAE (Session 1

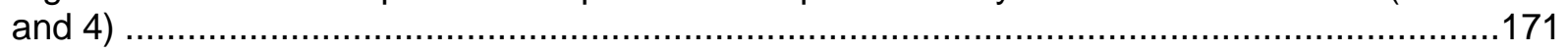

Figure 42: ERPs Source Location (100, 200, $300 \& 400 \mathrm{~ms}$ ) Patient 3 - Binaural $\mathrm{f}_{\mathrm{o}}$ (Session 1

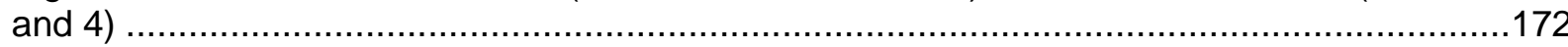

Figure 43: ERPs Source Location (100, 200, 300 \& $400 \mathrm{~ms})$ Patient 9 - Binaural 396Hz

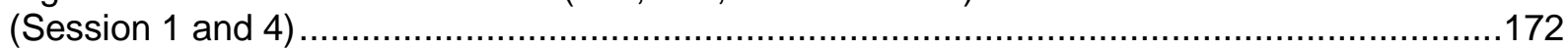

Figure 44: ERPs Source Location (100, 200, 300 \& $400 \mathrm{~ms}$ ) Patient 5 - TRT (Session 1 and 4)

Figure 45: ERPs Source Location (100, 200, 300 \& $400 \mathrm{~ms})$ Patient 7 - EAE (Session 1 and 4) 


\section{TABLES}

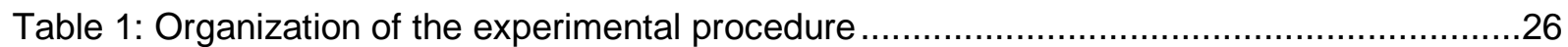

Table 2: Number of Patients who completed the 4 sessions per Therapy ...............................35

Table 3: Number of Patients who completed the 4 sessions of Binaural Therapy.....................36

Table 4: Tinnitus perception scale based on CIA.EVO total score calculation ...........................37

Table 5: Depression and Anxiety scale based on HADS total score calculation ........................37

Table 6: Average and standard deviation calculation from the evolution of Tinnitus perception

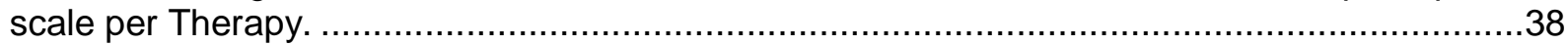

Table 7: Percentage of people versus Pulse evolution per therapy .....................................41

Table 8: Difference between session 4 and session 1: time and frequency parameters selection

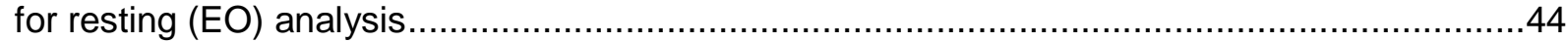

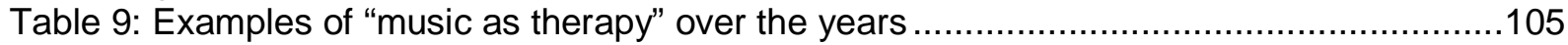

Table 10: Number of patients, average and standard deviation calculation from the evolution of

Tinnitus perception scale per Therapy and Initial CIA.EVO. ..............................................128

Table 11: Average and standard deviation calculation from the evolution of Anxiety perception

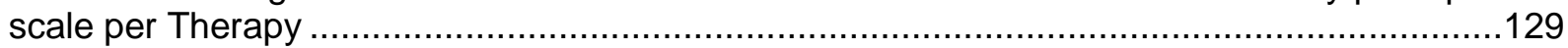

Table 12: Number of patients, average and standard deviation calculation from the evolution of

Anxiety scale per Therapy and Initial HADS-A. .............................................................129

Table 13: Average and standard deviation calculation from the evolution of Depression scale

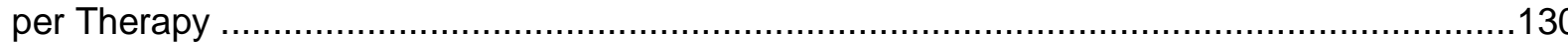

Table 14: Number of patients, average and standard deviation calculation from the evolution of

Depression scale per Therapy and Initial HADS-D.......................................................130

Table 15: Frequency domain parameters SESSION 4 - SESSION 1 difference for EO Binaural

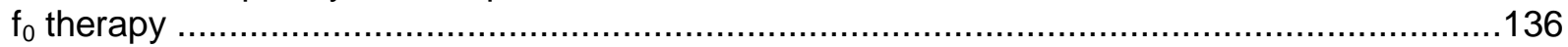

Table 16: Time domain parameters SESSION 4 - SESSION 1 difference for EO Binaural $f_{0}$

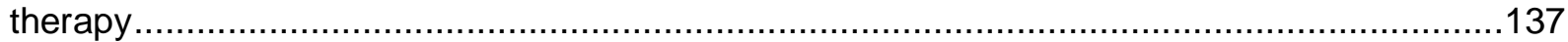

Table 17: Frequency domain parameters SESSION 4 - SESSION 1 difference for EO TRT

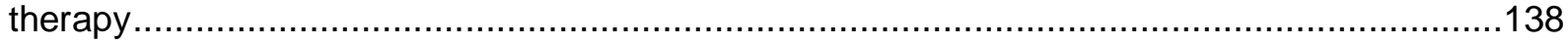

Table 18: Time domain parameters SESSION 4 - SESSION 1 difference for EO TRT therapy

Table 19: Frequency domain parameters SESSION 4 - SESSION 1 difference for EO EAE

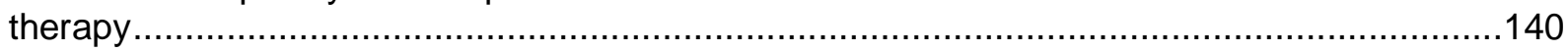

Table 20: Time domain parameters SESSION 4 - SESSION 1 difference for EO EAE therapy

Table 21: Frequency domain parameters SESSION 4 - SESSION 1 difference for EO RS

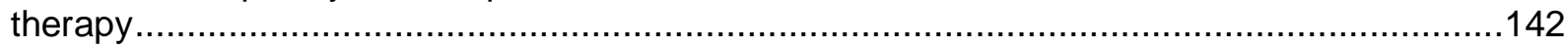

Table 22: Time domain parameters SESSION 4 - SESSION 1 difference for EO RS therapy 142

Table 23: Difference between session 4 and session 1: time and frequency parameters

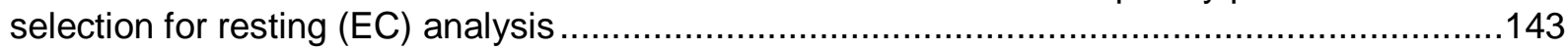

Table 24: Frequency domain parameters SESSION 4 - SESSION 1 difference for EC Binaural

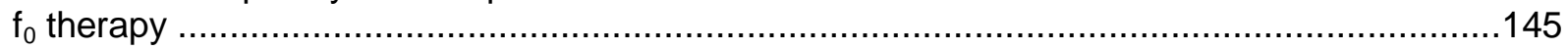

Table 25: Time domain parameters SESSION 4 - SESSION 1 difference for EC Binaural $f_{0}$

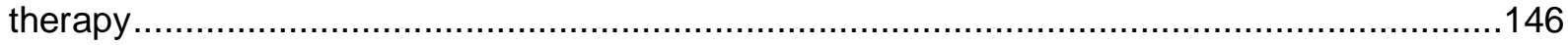

Table 26: Frequency domain parameters SESSION 4 - SESSION 1 difference for EC TRT

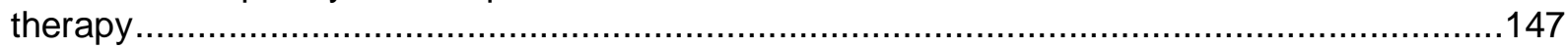

Table 27: Time domain parameters SESSION 4 - SESSION 1 difference for EC TRT therapy

Table 28: Frequency domain parameters SESSION 4 - SESSION 1 difference for EC EAE therapy 
Table 29: Time domain parameters SESSION 4 - SESSION 1 difference for EC EAE therapy

Table 30: Frequency domain parameters SESSION 4 - SESSION 1 difference for EC RS therapy

Table 31: Time domain parameters SESSION 4 - SESSION 1 difference for EC RS therapy 151

Table 32: Difference between session 4 and session 1: time and frequency parameters selection for therapy condition analysis

Table 33: Frequency domain parameters SESSION 4 - SESSION 1 difference for Acoustic

Therapy - Binaural $396 \mathrm{~Hz}$.

Table 34: Time domain parameters SESSION 4 - SESSION 1 difference for Acoustic Therapy -

Binaural $396 \mathrm{~Hz}$ 155

Table 35: Frequency domain parameters SESSION 4 - SESSION 1 difference for Acoustic

Therapy - TRT...

Table 36: Time domain parameters SESSION 4 - SESSION 1 difference for Acoustic Therapy -

TRT.

Table 37: Frequency domain parameters SESSION 4 - SESSION 1 difference for Acoustic

Therapy - EAE

Table 38: Time domain parameters SESSION 4 - SESSION 1 difference for Acoustic Therapy -

EAE

Table 39: Difference between session 4 and session 1: time and frequency parameters selection for GAv analysis.

Table 40: Frequency domain parameters SESSION 4 - SESSION 1 difference for GAv ERP -

Binaural $\mathrm{f}_{0}$ Therapy

Table 41: Time domain parameters SESSION 4 - SESSION 1 difference for GAv ERP -

Binaural $\mathrm{f}_{0}$ Therapy

Table 42: Frequency domain parameters SESSION 4 - SESSION 1 difference for GAv ERP -

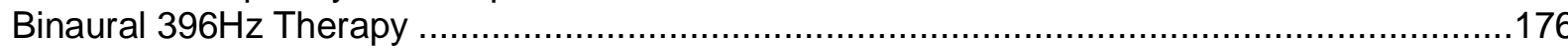

Table 43: Time domain parameters SESSION 4 - SESSION 1 difference for GAv ERP -

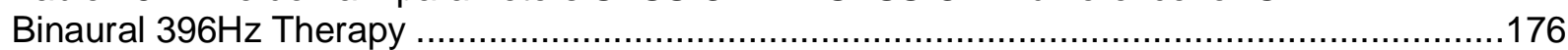

Table 44: Frequency domain parameters SESSION 4 - SESSION 1 difference for GAv ERP -

TRT Therapy

Table 45: Time domain parameters SESSION 4 - SESSION 1 difference for GAv ERP - TRT

Therapy.

Table 46: Frequency domain parameters SESSION 4 - SESSION 1 difference for GAv ERP -

EAE Therapy

Table 47: Time domain parameters SESSION 4 - SESSION 1 difference for GAv ERP - EAE

Therapy 
ANNEXES 


\section{ANNEX A: MUSIC AS A THERAPY IN EARLY HISTORY}

The following table is a summary of an extract from the final thesis of the Master of Advanced Studies ${ }^{30}$ from the same author of this PhD thesis (Tavira Sanchez, 2010).

\begin{tabular}{|c|c|c|c|}
\hline ORIGIN & PERIOD & REFERENCE & USE \\
\hline Shamans & $\begin{array}{c}\text { Upper } \\
\text { Paleolithic }\end{array}$ & $\begin{array}{l}\text { Today: Africa \& } \\
\text { South America }\end{array}$ & - Wisdom or healing \\
\hline $\begin{array}{l}\text { Aboriginal } \\
\text { Australians }\end{array}$ & 40.000 B.C & Didgeridoo & $\begin{array}{l}\text { - Healing or enter other states of } \\
\text { consciousness }\end{array}$ \\
\hline Hinduism & $\approx 1.750$ B.C & India & $\begin{array}{l}\text { - Healing } \\
\text { - Attain spiritual essence }\end{array}$ \\
\hline Egypt & $\approx 1.500$ B.C. & $\begin{array}{l}\text { Medical } \\
\text { papyrus }\end{array}$ & - Feminine fertility \\
\hline $\begin{array}{l}\text { Greek } \\
\text { Antiquity }\end{array}$ & $\approx 530$ B.C & $\begin{array}{l}\text { Pythagoreans } \\
\text { School, Croton }\end{array}$ & $\begin{array}{l}\text { - "Musical medicine" Method: } \\
\text { secrets of psychic transmutation } \\
\text { and healing through. } \\
\text { - Sound was considered essential } \\
\text { element of the universe. }\end{array}$ \\
\hline Buddhism & $\begin{array}{c}5^{\text {th }} \text { Century } \\
\text { B.C }\end{array}$ & Tibetan bowls & $\begin{array}{l}\text { - deep relaxation, stress reduction } \\
\text { - holistic healing, chakra balancing }\end{array}$ \\
\hline Daoism & $5^{\text {th }}$ Century & $\begin{array}{l}\text { Dr. Sun Si Mao, } \\
\text { China }\end{array}$ & $\begin{array}{l}\text { - "Fall in love with the organs" } \\
\text { practice: using } 6 \text { sounds } \\
\text { associated with human body } \\
\text { organs which resonance balance } \\
\text { their vital energy }\end{array}$ \\
\hline Africa & $7^{\text {th }}$ Century & Yoruba tribe & - Religious senses (reincarnation) \\
\hline
\end{tabular}

Table 9: Examples of "music as therapy" over the years

${ }^{30}$ DEA: Diploma de Estudios Avanzados in Spanish 


\section{ANNEX B: TINNITUS MODELS}

- The Jastreboff neurophysiological model suggests that, in tinnitus, the links between the elements of the CNS are governed by classical conditioning or associative learning. It highlights emotional processing as the major factor in generating tinnitus distress and promotes habituation as the means of reducing that distress (Baguley, et al., 2013). The goal is to reach the stage at which, although patients may perceive tinnitus as unchanged when they focus on it, they are otherwise not aware of tinnitus (Jastreboff, et al., 1996).

- Rauschecker et al. (2010) proposed another tinnitus model based on noise cancelation mechanism in which efferent projections from the subcallosal area are involved in the suppression of tinnitus signal as a sensory input at the thalamic level of brain processes. While a tinnitus signal originates from lesioninduced plasticity of the auditory pathways, it can be tuned out by feedback connections from limbic regions, which block the tinnitus signal from reaching auditory cortex (Rauschecker, et al., 2010).

- The integrative model conceptualizes that "tinnitus core" subnetworks incorporate neurophysiological model and noise canceling process. The discussion theorizes that minimal brain areas (auditory cortex, inferior parietal area, and ventromedial prefrontal / frontopolar cortex) jointly activate to achieve the conscious perception of tinnitus. The hypothesis assumes that separable tinnitus characteristics can be extracted by evaluating resting-state magnetic and electrical studies toward the evaluation of specific characteristics and control of other parameters. Moreover, it has been theorized that communications within different subnetworks would take place in hubs, which are defined as brain regions that simultaneously participate in various brain networks and can be involved in distinct subnetworks at discrete oscillatory frequencies (De Ridder, et al., 2014).

- The Neurofunctional Tinnitus Model hypothesizes that the perception of sound fundamentally depends on the allocation of attentional resources via frontal cortex, which in turn, depends on the cognitive-emotional value and the relevance of the phantom stimulus to the context. High cognitive-emotional value of sensory stimuli may allocate adequate attentional resources and trigger a topdown suppression process that acts on the thalamus noise-canceling mechanism and may lead to the awareness perception of the tinnitus phantom sound (Ghodratitoostani, et al., 2016). Structurally, the Neurofunctional Tinnitus Model includes the peripheral auditory system, the thalamus, the limbic system, brainstem, basal ganglia, striatum, and the auditory along with prefrontal cortices. Functionally, the model includes presence of continuous or intermittent abnormal signals at the peripheral auditory system or midbrain auditory paths. 
ANNEX C: TINNITUS DIAGNOSTIC AND THERAPEUTIC MANAGEMENT OF TINNITUS ${ }^{31}$

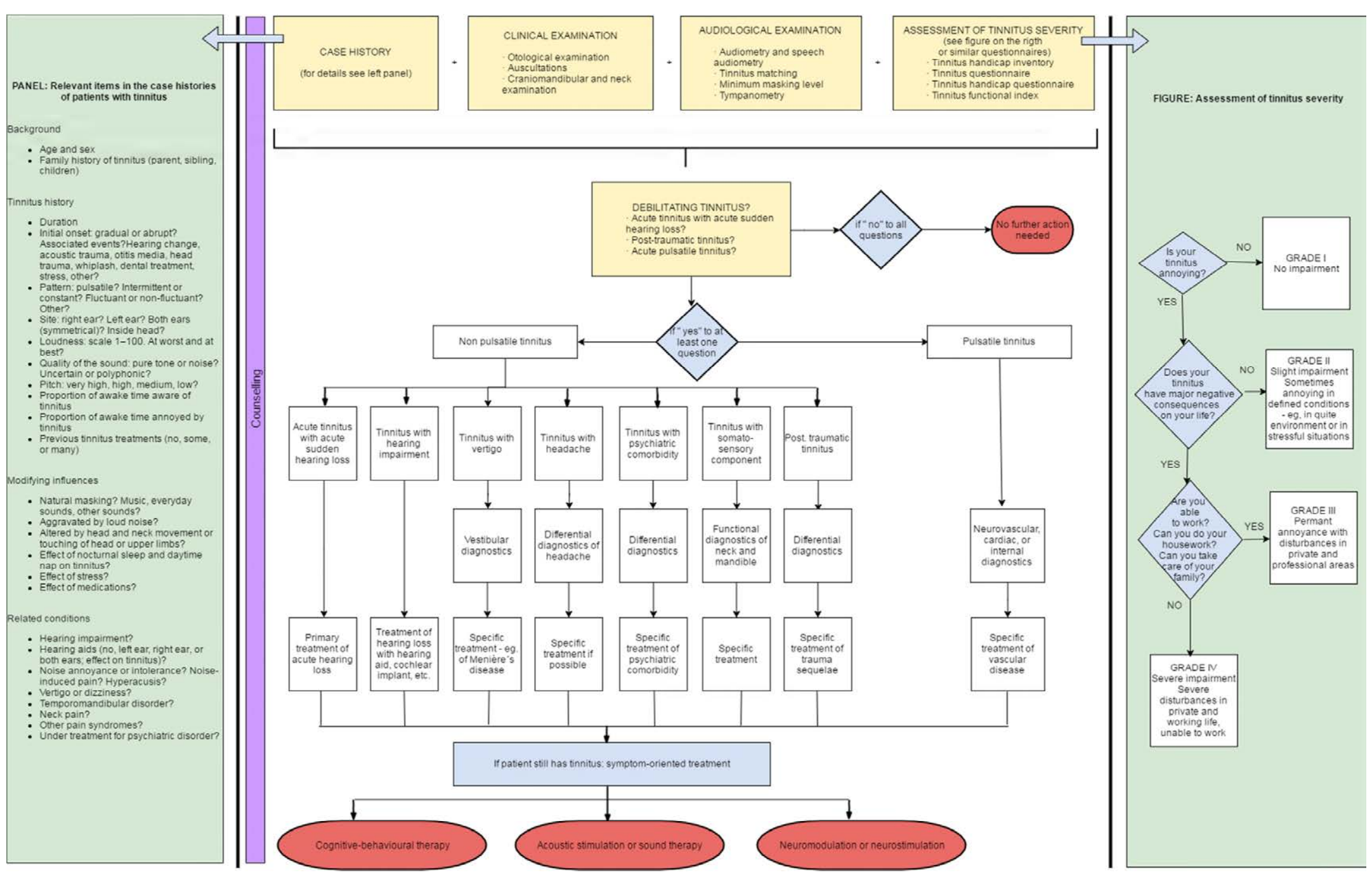

Figure 15: Tinnitus diagnostic and therapeutic steps versus specific conditions

${ }^{31}$ Modified by Langguth (2013) with permission from the Tinnitus Research initiative (http://www.tinnitusresearch.org/index.php/for-clinicians/diagnostic-flowchart) 


\section{ANNEX D: TINNITUS DEMOGRAPHICS}

Tinnitus can be perceived by people of all ages, and with normal hearing or with deafness. In average, the probability of a person to suffer from tinnitus increases with age and degree of hearing loss: around $12 \%$ of patients suffering from tinnitus are over 60 years, while only $3 \%$ are between 20 and 30 years (Parazzini, et al., 2011). Male sex has been identified as more relevant risk factor for tinnitus (Langguth, et al., 2013).

For Europe, one example is a recent data collected between 2006 and 2010 as part of the UK Biobank resource showed a $16,9 \%$ prevalence for adults ages 40 to 69 years (Dawes, et al., 2014).

Figure 15 shows age and sex-specific trends in chronic tinnitus from the U.S. National Health Interview Survey in 1995 (Hoffman \& Reed, 2004).

Age and Sex in chronic tinnitus U.S. 1995

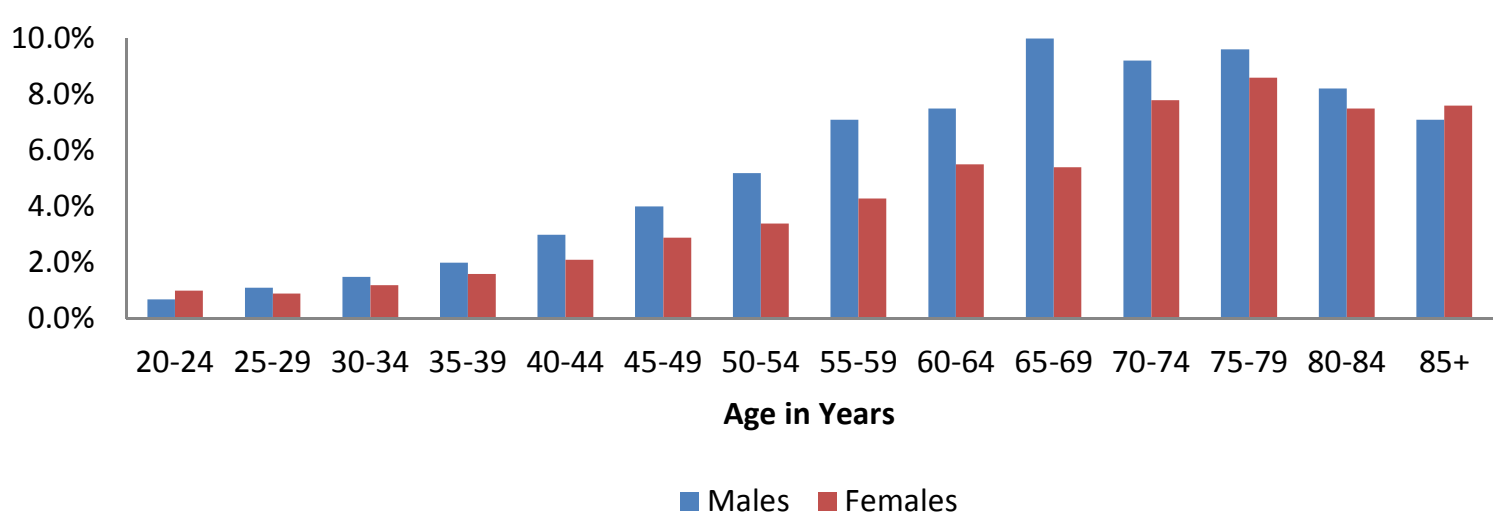

Figure 16: Age and sex-specific trends in chronic tinnitus from the U.S. National Health Interview Survey in 1995

In $3 \%$ of the total affected population, tinnitus is intense enough to deteriorate the quality of life (Páez, 2006; Espinosa-Sánchez, 2014; Sweetow, 2013), involving sleep disturbance, restricted working capacity, difficulty to focus attention (Eggermont \& Roberts, 2004; Weisz, et al., 2004), or contribute to psychological disorders such as depression, suicide ideation, posttraumatic stress disorder, anxiety and anger (Noble \& Tyler, 2007).

It is generally agreed that most people with tinnitus have at least a mild hearing loss (Stouffer \& Tyler, 1990) though if comprehensive audiological services are used to fit hearing aids, there is a stronger probability that the subject with tinnitus will derive benefit from their hearing aids to treat their tinnitus (Kochkin, et al., 2011).

The number of people with hearing loss is more than those living with Parkinson's, epilepsy, Alzheimer's and diabetes combined. According to the World Health Organization (WHO) estimates, the number of people with disabling hearing impairment 
increased from 42 million in 1985 to about 360 million in 2011 (Olusanya, et al., 2014) being children and elderly the greatest in the Asian Pacific area, southern Asia and subSaharan Africa (WHO, 2012).

Tinnitus prevalence is expected to continue to increase due to demographic developments and an increase in professional and leisure noise exposure.

According to Royal National Institute for Deaf People (RNID), people with hearing loss in UK will increase from 11 million in 2015 to 15.6 million in 2035 (Actiononhearingloss, 2016). On the other hand, nearly 324.000 new cases of significant tinnitus (sigT) are expected to occur in England between 2012 and 2021 (Martinez, et al., 2015). 


\section{ANNEX E: BINAURAL BEATS SUMMARY INFORMATION}

- It was discovered by Dove in 1841 and popularized by Oster (Oster, 1973)

- It may have a direct effect on the neural frequencies, tested through EEG and explained as spontaneous synchronization (Curtis, 2007; Vernon, et al., 2014).

- It could be a listening challenge which makes the auditory system resolves a conflict of binaural perception by increasing the communication between the two auditory cortexes (Gao, et al., 2014; Solcà, et al., 2016; Becher, et al., 2014).

- Among others, some of the effects related are

- Improving memory capacity. Example at $9,55 \mathrm{~Hz}$ with two tones of frequencies of $230 \mathrm{~Hz}$ and $220.45 \mathrm{~Hz}$ (Kraus \& Porubanová, 2015) or $5 \mathrm{~Hz}$ for verbal memory (Ortiz, et al., 2007)

- Improving attention or vigilance. Examples at delta/theta range (Kennel, et al., 2010), $7 \mathrm{~Hz}$ and $16 \mathrm{~Hz}$ (Stough, et al., 2001) or eliminating the attentional blink (Reedijk, et al., 2015).

- Empowering creativity. Example at both $10 \mathrm{~Hz}$ and $40 \mathrm{~Hz}$, affecting performance in the divergent, but not convergent thinking tasks (Reedijk, et al., 2013)

- Reducing stress or anxiety. Examples at $10 \mathrm{~Hz}$ (Norhazman, et al., 2014; Young, et al., 2014; Weiland, et al., 2011; Garcia-Argibay \& Santed, 2019) or delta/theta range (Le Scouarnec, et al., 2001; Padmanabhan, et al., 2005)

- Changing mood states (Chaieb, et al., 2015). Examples at $7 \mathrm{~Hz}$ (Wahbeh, et al., 2007), delta $(0.1-4 \mathrm{~Hz})$ (Wahbeh, et al., 2007) and beta range (13$30 \mathrm{~Hz}$ ) (Lane, et al., 1998), decreasing in tension, anxiety, confusion, and fatigue but increase in depression with less negative impact in beta.

- There is not much literature about tinnitus treatment. One research was done at $10 \mathrm{~Hz}$ and carrier wave between $50 \mathrm{~Hz}$ and $100 \mathrm{~Hz}$ (David, et al., 2010), where the mean disturbance rating from 26 patients was reduced from 5.81 to 3.06 (THI and stress-related symptoms questionnaires). 
ANNEX F: SAMPLE OF ACOUSTIC THERAPIES ANALYSIS
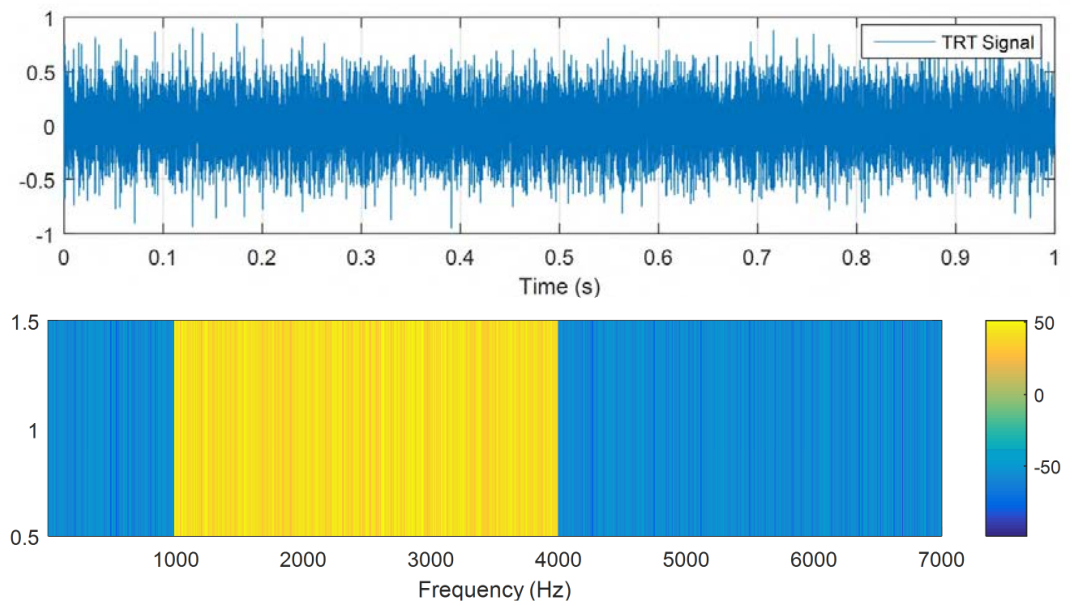

Figure 17: Sample of TRT signal, time and frequency analysis
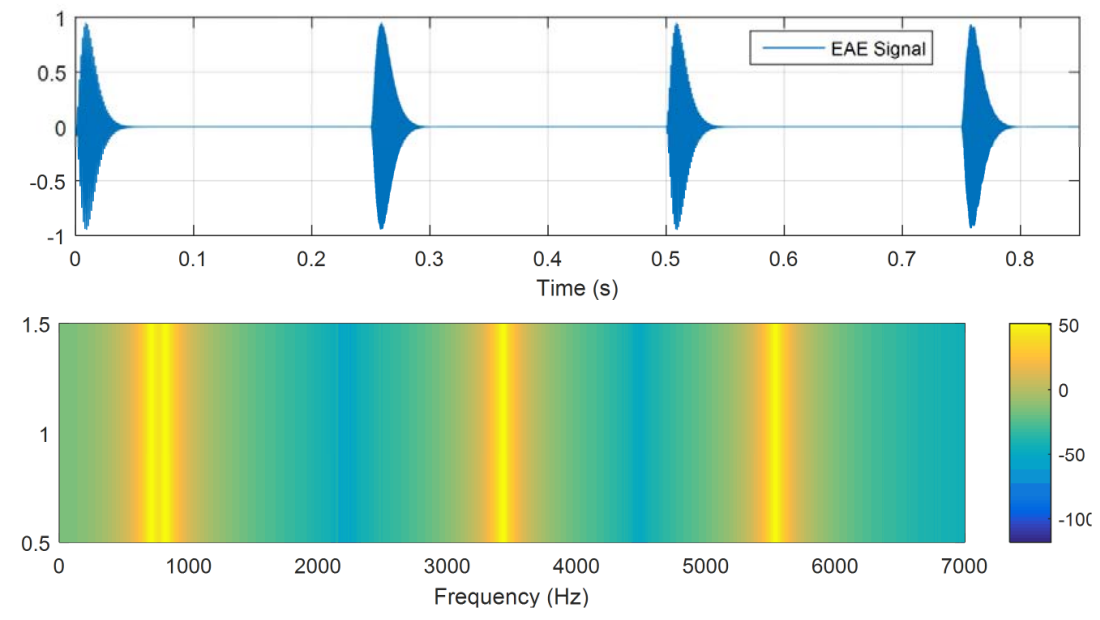

Figure 18: Sample of TEAE signal: time and frequency analysis
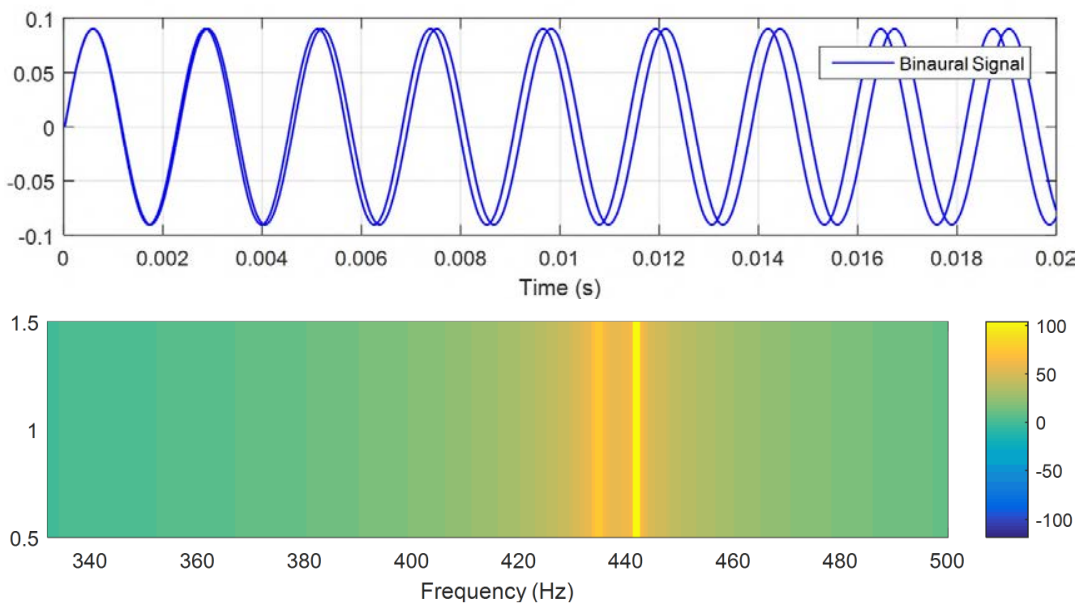

Figure 19: Sample of Binaural signal: time and frequency analysis 


\section{ANNEX G: AUDIO PLAYER TECHNICAL DETAILS AND DISTORTION}

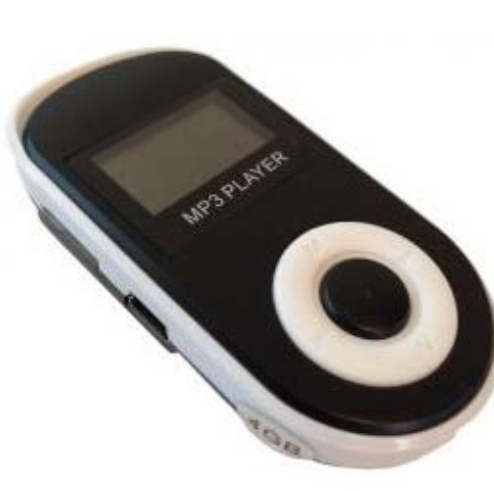

\begin{tabular}{|c|c|}
\hline \multicolumn{2}{|c|}{ Portable audio player (MP3/USB/RADIO FM) } \\
\hline MODEL & MP3DIS \\
\hline BRAND & FUXION \\
\hline SKU & XX314EL01RO80LMX \\
\hline SIZE $(\mathrm{L} \times \mathrm{H} \times \mathrm{W} \mathrm{cm})$ & $22 \times 16.5 \times 4.5$ \\
\hline WEIGHT $(\mathrm{Kg})$ & 0.1 \\
\hline COLOR & Black \\
\hline $\begin{array}{l}\text { ADAPTER } \\
\text { Electrical characteristics }\end{array}$ & $\begin{array}{l}\text { Input: ca } 100-240 \mathrm{~V} ; 0,1 \mathrm{~A} 50 / 60 \mathrm{~Hz} \\
\text { Output: } 5 \pm 0,5 \mathrm{~V} ; 200 \pm 50 \mathrm{Ma}\end{array}$ \\
\hline \multicolumn{2}{|c|}{ HEADPHONES } \\
\hline Bandwidth $(\mathrm{Hz})$ & $12-22.000$ \\
\hline Sensitivity $(\mathrm{dB} / \mathrm{mW})$ & 100 \\
\hline Power input (mW) & 5 \\
\hline Impedance $(\Omega)$ & 32 \\
\hline Audio interface & Jack Plug 3,5 mm \\
\hline
\end{tabular}

Figure 20: Audio player provided to patient

(a)

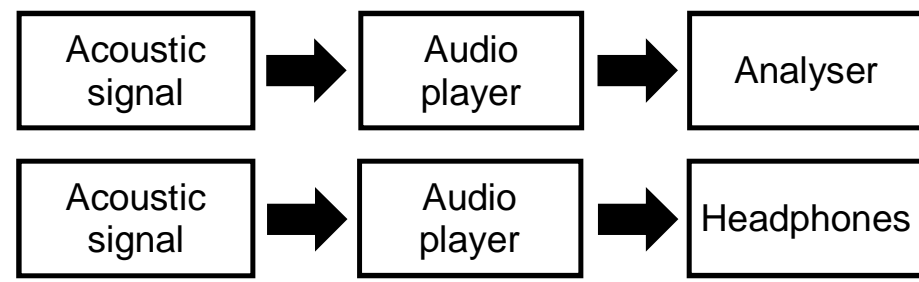

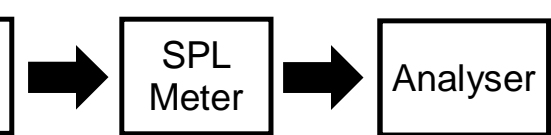

Figure 21: Assembly to evaluate the audios connecting (a) the audio player directly with the Analyser and (b) through Headphones and SPL Meter ${ }^{32}$

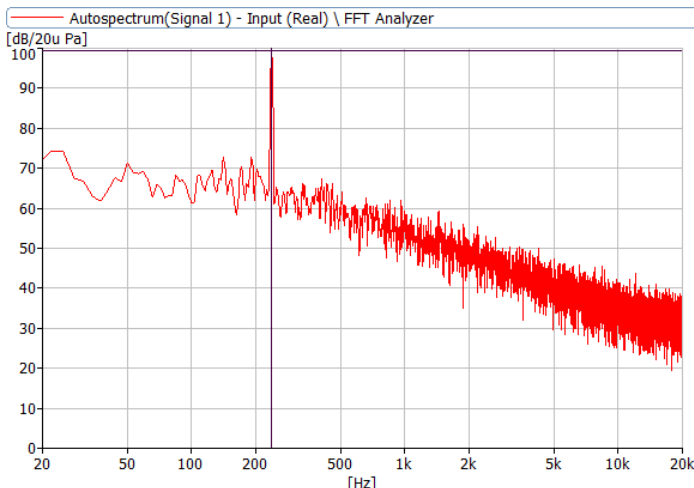

(a)

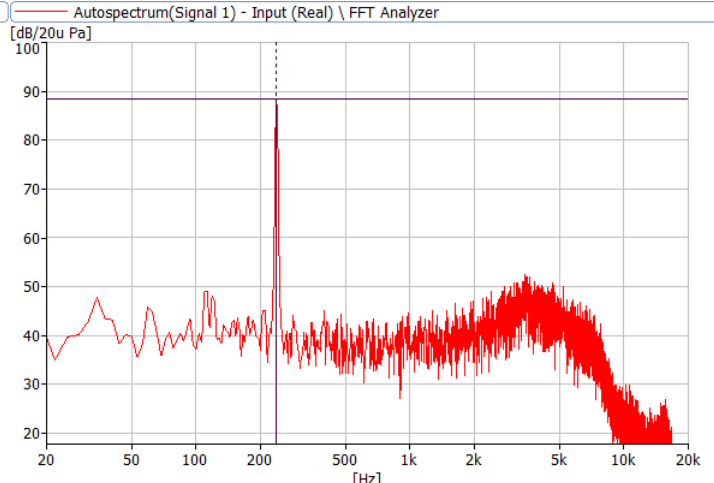

(b)

Figure 22: Frequency response of the audio player playing a pure tone at $232 \mathrm{~Hz}$ connecting (a) it directly with the Analyser and (b) through Headphones and SPL Meter

${ }^{32}$ SPL Meter: Digital Sound Level Meter from Radioshack (see technical specification in the following link: https://www.manualslib.com/manual/610760/Radio-Shack-Sound-Level-

Meter.html?page=5\#manual). It was used with weighting $C$ (all range) and connected to the analyser with a coaxial cable.

Analyser: Pulse Brüel \& Kjær (more info at https://www.bksv.com/en/products/PULSE-analysis-software) 


\section{ANNEX H: UNCERTAINTY CALCULATION}

The $\mathrm{GUM}^{33}$ classifies elemental uncertainties in two categories (Ratcliffe \& Ratcliffe, 2015):

1. Type A (random): uncertainties that can be evaluated by statistical methods. Some examples: repeatability, thermal stability and noise.

2. Type B (systematic): any other uncertainty evaluated by any other means, such as resolution, scale size or quantization, hysteresis, common mode voltage, installation, nonlinearity (or linearity), spatial variation, loading, zero offset and sensitivity.

Those uncertainties generate the combined uncertainty $\left(u_{c}\right)$, and jointly with the coverage factor $(k)^{34}$, it is calculated the extended uncertainty $(U)$ :

$$
\text { where } \quad u_{c}=\sqrt{u_{1 A}^{2}+u_{1 B}^{2}+u_{2 A}^{2}+u_{2 B}^{2}}
$$

It is considered uncertainty type $\mathrm{A}$

$u_{1 A}$ repeatability of the audio player

$u_{2 A}$ repeatability of the system headphones / SPL

$$
u_{A}=s(\bar{x}) \cdot t_{n-1 ; 95,45 \%}
$$

where

$$
s(\bar{x})=\frac{s(x)}{\sqrt{n}} ; s(x)=\sqrt{\frac{1}{n-1} \sum_{i=1}^{n}\left(\bar{x}-x_{i}\right)^{2}}
$$

Having ten $(\mathrm{n}=10)$ measurements from a tone

$$
\begin{aligned}
& u_{1 A}=0,08 \cdot 2,32=\mathbf{0}, 18 \boldsymbol{d} \boldsymbol{B} \\
& u_{2 A}=0,13 \cdot 2,32=\mathbf{0}, 31 \boldsymbol{d} \boldsymbol{B}
\end{aligned}
$$

It is defined uncertainty type B

$u_{1 B} \approx 0$ not possible to calculate it with the current equipment and it is considered as null because it is a simple electrical device

$$
u_{2 B}=\sqrt{u_{2 B \text { Pos }}^{2}+u_{2 B T e m}^{2}+u_{2 B P r e}^{2}+u_{2 B C a l}^{2}}=1,8 \mathbf{d B}
$$

where

$u_{2 B P o s}$ headphone spatial variation (Position) $=1 \mathrm{~dB}^{35}$

$u_{2 B C a l}$ SPL Calibration $=1,5 \mathrm{~dB}^{36}$

$u_{2 B T e m}$ environmental Temperature $\approx 0$ considered as null because it has not varied much from the standard conditions

$u_{2 B \text { Pre }}$ environmental Pressure $\approx 0$ considered as null because it has not varied much from the standard conditions

Concluding,

$$
U \cong 2 \cdot 1,84=3,68 d B
$$

\footnotetext{
${ }^{33}$ It was first published in 1992 by International Organization for Standardization / Bureau International des Poids et Mesures (ISO / BIPM)

${ }^{34}$ Usually $k=2$ providing a level of confidence of approximately $95,45 \%$ (from the $t$-student table) with infinitives degrees of freedom

35 Typical uncertainty due to the measurement position (Instituto Nacional de Seguridad, Salud $y$ Bienestar en el Trabajo http://calculadores.insht.es:86/Incertidumbredelruido/Introducci\%C3\%B3n.aspx)

${ }^{36}$ Class 2 SPL Norm IEC 61672-1:2002
} 


\section{ANNEX I: BRAIN OSCILLATIONS QUANTIFIED IN TINNITUS}

- Slow brain rhythms (delta $\delta$ and theta $\theta$ ). Slow wave activity, either in $\delta$ or $\theta$ bands, has been frequently related to many neuro-pathologies such as tumours, strokes, depression, schizophrenia, and Alzheimer's disease. It has been demonstrated (Balkenhol, et al., 2013) demonstrated that tinnitus loudness and tinnitus-related distress are associated with delta band oscillations. Interestingly, they found that tinnitus-related distress correlates with delta band power at recording sites different from those where significant correlation between delta rhythms and tinnitus loudness was found. Furthermore, patients suffering from tinnitus and with high related distress showed higher theta oscillations than those with low related distress. In the same vein, it has been argued (Adjamian, 2014) that delta band rhythms over the auditory cortex are a signature of tinnitus.

- Alpha $(\alpha)$ band rhythms. According to Elgoyhen et al. (2015), the majority but not all EEG evaluations over the temporal cortex of people experiencing tinnitus show a reduction of $\alpha$ power. Houdayer et al. (2015) also found that tinnitus suffers showed low $\alpha$ power over the left inferior parietal lobule in EO condition, and low a power over the left inferior temporal lobule in EC condition. In this respect, Vanneste et al. (2014) had been previously hypothesized that healthy individuals show high $\alpha$ synchronization when their attention is focused internally; in contrast, tinnitus suffers redirect their attention towards their tinnitus during mind wandering, leading to low $\alpha$ synchronization.

- Beta $(\beta)$ band rhythms. EEG synchronization of $\beta$ band rhythms has been detected as indicative of a chronic dysrhythmia of thalamus cortical circuits (following auditory deafferentation), and tinnitus-related emotional distress (Meyer, et al., 2014).

- Gamma ( $\gamma$ ) band rhythms. Auditory perception is reflected as $\gamma$-band oscillations over the auditory cortex, and hence, they seem to be strongly related to the perception of tinnitus. Most EEG studies have found that chronic tinnitus is correlated with increased $\gamma$-band activity in the contralateral auditory cortex, while some others have demonstrated that $\gamma$-band rhythms are enhanced in accordance with subjective tinnitus loudness. On this evidence, abnormal $\gamma$ synchronous oscillations should be considered a neural signature of chronic tinnitus percept. Gamma activity may be also related to attention directed towards and emotions generated by tinnitus percept (Elgoyhen, et al., 2015; Adjamian, 2014; Meyer, et al., 2014; Balkenhol, et al., 2013). 


\section{ANNEX J: EEG ANALYSIS}

Methodological pathway to analyse EEG signals in order to evaluate the effects of acoustic therapies used to reduce the tinnitus discomfort.

\section{Spontaneous Activity}

- Absolute Power

○ Delta $\delta(0.1-4 \mathrm{~Hz}) /$ Theta $\theta(4-8 \mathrm{~Hz})$ : Tinnitus-related distress

- Alpha $\alpha(8-13 \mathrm{~Hz})$ : Full attention toward tinnitus

- Beta $\beta(13-30 \mathrm{~Hz})$ : Emotional distress

- Gamma y $(>30 \mathrm{~Hz})$ : Tinnitus perception \& loudness

- Relative Power, Coherence, Entropy

- Time-frequency Analysis: STFT and Wavelets

\section{Evoked Activity}

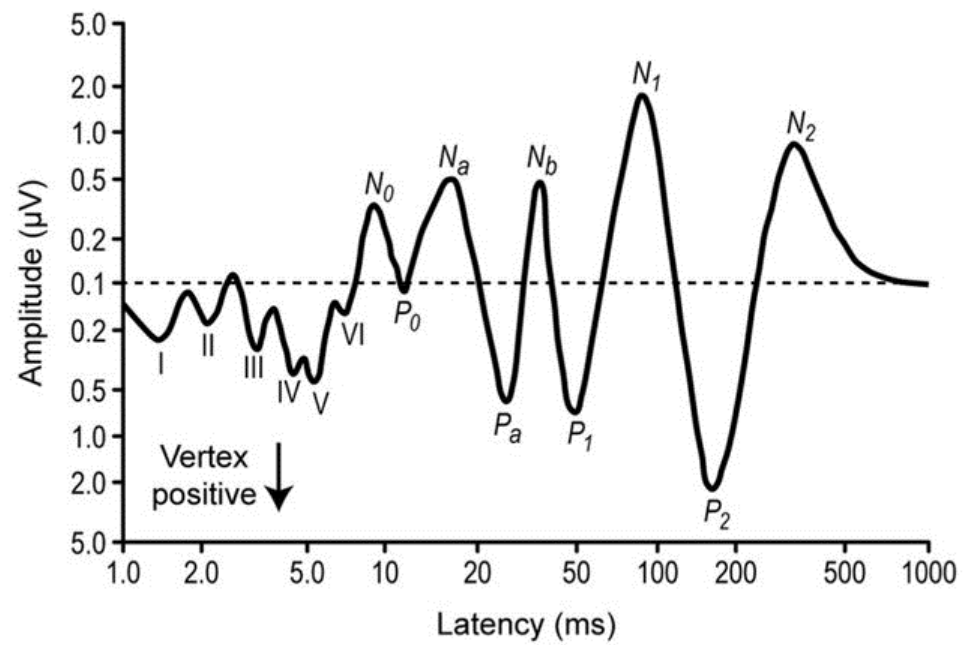

Figure 23: Diagrammatic representation of the auditory evoked potential components ${ }^{37}$

- Typically, ERPs are assessed by their amplitude (generally measuring maximum peak, distance from peak to peak, area under the curve), latency (early, medium, and late), location, and ratio between different components. Clinically, component $\mathrm{N} 1$ has been employed as indicator of neural deficits such as cerebrovascular disease, schizophrenia, and tinnitus. P3 is also a very commonly used component because it reflects high order brain functions, fundamentally cognitive processes (Adjamian, 2014). What follows is an account of the most significant and interesting findings of research based on ERPs, and undertaken to evaluate the perceptual and cognitive state of tinnitus suffers.

\footnotetext{
${ }^{37}$ There are many components generated from an auditory stimulus. As shown in Figure 26, the components from the first $10 \mathrm{~ms}(\mathrm{I}-\mathrm{VI})$ correspond to the auditory brainstem responses, around 20-50 ms correspond to the middle latency responses, and 150-250 ms correspond to the activation of the frontal cortex (Pérez-González \& Malmierca, 2014). Redrawn from Picton, et al. (1974). Most of those components are not perceived within the EEG signal adquired due to the noise or the nature itself of the process. That is why a selection of a particular ERPs are chosen.
} 
- N1 and P2 have been the most commonly used components to quantify the effects of tinnitus at a cortical level. Delb et al. (2008) found significant differences between healthy individuals, and patients with both high and low tinnitus related distress. N1 amplitudes found to be larger in healthy people, in comparison with those with tinnitus. Furthermore, tinnitus suffers with low distress had larger N1 components than those with high distress. Similarly, the synchronization stability of $\mathrm{N} 1$ was higher in persons without tinnitus than those with tinnitus. Authors concluded that the attention of patients with high tinnitus related distress is extremely directed towards tinnitus, in comparison with patients with low distress and healthy individuals. Regarding the qualification of $\mathrm{N} 1$ latency, Houdayer et al. (2015) identified shorter N1 and P2 latencies in subjects with tinnitus than those without tinnitus. Lately, it is important to mention that findings of Zeng et al. (2011) differ from those of Delb et al., but agree with Houdayer et al. Zeng and colleagues evaluated the efficiency of lowrate electric stimulation as tinnitus abolisher via N1 comparisons. Authors reported that $\mathrm{N} 1$ was reduced and delayed when tinnitus was suppressed through electrical stimulation (Zeng, et al., 2011 ; Delb, et al., 2008; Houdayer, et al., 2015).

- P3 is another quite common component employed to characterize tinnitus nature. $\mathrm{P} 3$ is mostly evoked during decision-making protocols to study cognitive processes. As was aforementioned, tinnitus suffers are caught in a cycle of increasing perceptual and emotional negativity and decreasing cognitive functioning. Attias et al. in 1993, and later in 1995 and 1996, found that patients with chronic tinnitus showed smaller P3 components, in comparison with those of healthy people. Authors argued that it could be the result of a deficiency in the central auditory processing system due to tinnitus (Attias, et al., 1993; Attias, et al., 1995; Attias, et al., 1996). On the other hand, Gabr et al. (2011) found that patients with tinnitus showed P3 components with larger latencies than healthy people, possibly owing to the presence of depression and anxiety.

- More recently, auditory steady-state responses (ASSR) have been examined in age and hearing- level matched tinnitus and control groups. One remarkable example is the work of Roberts et al. (2012), who investigated brain changes induced by sensory training therapies in tinnitus suffer. Researchers observed that ASSR amplitude increased in the tinnitus group, whereas no changes were seen in the control group. Moreover, during intake tasks, ASSR phase between stimulus and response was around $-10^{\circ}$ in the control group, but no significant phase changes were detected in the tinnitus group. Another example is the work of Paul et al. (2014), who evoked ASSR at $500 \mathrm{~Hz}$ and $5 \mathrm{kHz}$. They reported that ASSR amplitude increased in the control group at both 
frequencies. However, ASSR amplitude only increased at $500 \mathrm{~Hz}$ in the tinnitus group (Paul, et al., 2014; Roberts, et al., 2012).

- ERPs can also be assessed (Kappenman \& Luck, 2012) through:

- Source localization techniques start from biophysical assumptions about the current flow through tissues of the head to find the scalp distribution of each obtained component.

- Principal component analysis confines as much information as possible into the smallest number of components in line with statistical significance of EEG data.

- Independent component analysis (ICA) splits apart information into different components that are statistically independent.

- In the time-frequency approach, EEG signals are decomposed into the sum of neural oscillations. After that, electrical properties such a power are investigated.

Induced Activity

- Event-Related Band Power Analysis: ERD-ERS Maps and Induced band power

- Event-Related Spectrum

- Coherence: Event-related coherence analysis, Phase-locking statistics an Eventrelated phase resetting

- Single-Trial Wavelet Analysis

- Multi-taper Analysis 


\section{ANNEX K: SIGNAL-PROCESSING TOOLS}

\section{Relative Power}

Amplitude, and thus power, of EEG signals depends on multiple non-physiological factors such as anatomical and physical properties of the brain, and surrounding tissues (skin, bone, dura-mater, and pia-mater). Therefore, absolute power presents higher variability than relative one. Relative power $(\mathrm{Pr})$ is defined as the ratio of absolute power $(\mathrm{Pa})$ in a single frequency band to either the total power or the power within certain bands (Equation 6). At present, majority of studies have made use of absolute power. As relative power shows minor variability, this estimate could be useful to clarify some controversial issues raised by Pierzycki et al. (2016).

$$
P_{r}(f)=\frac{P_{a}(f)}{\sum_{i} P_{a}\left(f_{i}\right)}
$$

\section{Coherence}

According to Bastiaansen and colleagues (2012), neural networks work by local nodes that operate in synchrony with each other, and through synchronization between different nodes of a network. Absolute and relative power are used to monitor synchronization level inside local nodes, whereas coherence between recording sites yields information concerning synchronization level among different nodes (Bastiaansen, et al., 2012). Namely, coherence is the correlation between EEG powers estimated in the same frequency band but in different recoding sites. Mathematically, EEG coherence is the ratio of cross-power spectral density $\left(S_{x y}\right)$, and power spectral densities of recording sites of interest $\left(S_{x x}\right.$ and $\left.S_{y y}\right)$. That is,

$$
\operatorname{Coh}_{x y}=\frac{\left|S_{x y}(f)\right|^{2}}{S_{x x}(f) S_{y y}(f)}
$$

\section{Time-Frequency Analysis}

Apart from time analysis mentioned so far, frequency analysis is also essential. The traditional tool to analyse signal frequencies is Fourier series and Fourier transform. However, Fourier analysis by itself has two technical shortcomings:

- EEG signals are considered a random process

- although timing properties are encoded in the phase portion of the transform, they are difficult to retrieve and interpret.

Historically, two main approaches have been proposed to extract both time and frequency information from quasi-stationary signals. These are:

- Short-Time Fourier Transform (STFT). It attempts to covert EEG waveforms into quasi-stationary signals by slicing them into segments that could be considered as a stationary process. Previous studies (Sörnmo \& Laguna, 2005) have demonstrated that EEG signals become increasingly non-stationary as its length 
increases. Interestingly, it was found that more than $90 \%$ of one-second segments showed Gaussian distribution, while less than $50 \%$ of eight-second segments had Gaussian distribution. In fact, slicing EEG signals into one-second segments is a common practice in brain-computer interfaces projects, what seems to be a good approach to consider EEG signals as stationary waveforms. However, there is another drawback of STFT: time-frequency trade-off. It is well known that time resolution reduces frequency resolution, and vice versa. As STFT has been used successfully in study cases where only high frequencies are of interest, this tool seems to be very feasible to analyse gamma band rhythms. As was mentioned, gamma band oscillations reflect perception and loudness of tinnitus.

- Time-Scale methods (Wavelet analysis). The principle lays down on analysing high frequency components within small time windows, and low frequency components within large time windows, what overcomes STFT drawbacks. Furthermore, Wavelet analysis is a time-scaled method, what exploits the EEG benefits: high time resolution. Wavelet analysis has proved to be a potential tool in electrophysiological signal processing, particularly in short duration signals such as evoked potentials and cardiac cycles. One important limitation is the selection of the most appropriate Wavelet family.

\section{Entropy}

EEG signals can be studied via stochastic models (as was described above) and dynamic systems. One way to analyse dynamic systems is chaos theory, which focuses on aperiodic behaviours and limited predictability. This seems to be a very good description of EEG patterns. Over the past few years, chaos theory has been used to study neural oscillations as they are: non-stationary, non-Gaussian, and non-linear. Particularly, entropy that is a concept that addresses randomness and predictability has become a common practice in epileptic seizure prediction (Freestone, et al., 2017). As entropy can be defined as the rate at which new information is produced, and tinnitus seems to result of abnormal neural synchronization, the effectiveness of acoustic therapies could be assessed through entropy analysis of EEG signals. Great entropy is associated with more randomness; therefore, low entropy could be reflecting such abnormal neural rhythmicity (Zavala-Yoe \& Ramirez-Mendoza, 2017). 


\section{ANNEX L: EVENT-RELATED (DE) SYNCHRONIZATION}

Event-related (de) synchronization (ERD/ERS) attempts to quantify the level of synchrony of the EEG signals. ERD/ERS mechanism appears in specific bandwidths and both processes may exist simultaneously (Pfurtscheller \& Lopes da Silva, 1999). ERD/ERS are commonly analysed in time by mapping band power changes. ERD/ERS maps allow visualizing the neural processing of the cerebral cortex from a few milliseconds to several seconds. This is a method that was proposed by Pfurtscheller and Lopes da Silva (1999), and which has been significantly enhanced by Graimann and colleagues (Graimann, et al., 2002; Graimann \& Pfurtschelle, 2006). The conventional method followed to obtain ERD/ERS maps is as follows.

1. all event-related trials are band-pass filtered, the amplitude mean of each trial is subtracted, and the amplitude samples are squared (Equation 11).

2. power samples are averaged across all trials (Equation 10) and average power in the reference $\left[r_{0}, r_{0}+k\right]$ is calculated (Equation 9).

3. samples are averaged over time to smooth data and reduce the variability.

4. the ERD/ERS maps is computed by

$$
\begin{aligned}
& \mathrm{ERD} / \mathrm{ERS}_{j}=\frac{P_{j}-R}{R} \times 100 \\
& R=\frac{1}{k+1} \sum_{r_{0}}^{r_{0}+k} P_{j} \\
& P_{j}=\frac{1}{N-1} \sum_{i=1}^{N} y_{i j} \\
& y_{i j}=\left(S_{i j}-\bar{S}_{j}\right)^{2}
\end{aligned}
$$

In particular, auditory ERD/ERS maps have been mainly investigated by Krause and colleagues (Krause, et al., 1994; Krause, 1999; Krause, 2003). Some of their findings are outlined as follows:

- Auditory ERD/ERS maps reflect cognitive and attentional processes, rather than the decoding of the auditory stimulus per se

- auditory stimulus processing is associated with alpha band synchronization between 10 and $12 \mathrm{~Hz}$

- auditory memory increases the level of synchronization of the neurons

- the comparison of sounds increases the level of desynchronization of the neurons

- auditory stimuli modify the level of neural synchronization in temporal and parietal lobes. 
Recently, EROs have been applied to give an insight into the dynamics of cortical reorganization induced in diverse medical contexts. For example, Caimmi et al. (2016) showed that the ERD/ERS analysis was a feasible method to measure the efficiency of robot-assisted upper-limb devices.

Although robots have been proposed for motor relearning in neuro-rehabilitation (particularly, after stroke episodes), it had not been demonstrated that robot-assisted therapies were as efficient as conventional ones. Authors made use of ERD/ERS maps to show that robotic devices activate sensory-motor areas in alpha and beta bands. Such activation is the one involved in any movement executed by an individual (Caimmi, et al., 2016). Another example is the estimation of EROs to quantify somehow pain perception. Activation of nociceptive fibres has been related to

- alpha ERD over somatosensory, motor, and visual areas

- beta ERD predominantly over the contralateral primary motor cortex

- gamma ERS over contralateral somatosensory cortex.

This investigation has been useful to understand sensory perception, subjective intensity, and behaviour of pain. Voluntary movements that prevent tissue damage during pain processing have been also investigated (Peng \& Tang, 2016).

As tinnitus is likely the result of increased neural synchrony, estimation of the levels of synchrony of the neural networks may be a feasible method to assess the effects of acoustic therapies to treat tinnitus. It had been exemplified above the versatility of ERD/ERS estimation to capture the dynamics of neural oscillations related to emotional, cognitive, perceptual, and motor events. 


\section{ANNEX M: COMMON TINNITUS QUESTIONNAIRES}

- VAS is used to quantify subjectively certain sensations of the patients such as pain. This sliding scale ranges from lack of sensation to extreme feeling. Patients mark a point on the line that matches their sensation magnitude (Haefeli \& Elfering, 2006; Kersten, et al., 2014).

- The questionnaires consist of a series of questions to identify the difficulties that patients are facing due to the tinnitus, and whether those difficulties are overcome after applying an acoustic therapy: tinnitus status, evolution and side effects. Typically, only three responses are provided in the questionnaires: yes, sometimes, and no (Powers \& dos Santos, 2015; Adamchic, et al., 2014). Some examples are the following:

- Langguth and his colleagues (Langguth, et al., 2007) recommended the use of several questionnaires used in many studies, although preferentially in Europe (Hall, et al., 2016; Müller, et al., 2016).

- The Tinnitus Sample Case History Questionnaire, which was designed to assess the most important Tinnitus characteristics and the Tinnitus history of patients (Landgrebe, et al., 2010).

- THI, which typically assessed Tinnitus-related impairment in daily life (Newman, et al., 1996; Newman, et al., 1998).

- The Tinnitus-Beeinträchtigungs-Fragebogen (Greimel, et al., 1999) which has an updated version called the Tinnitus Functional Index (Meikle, et al., 2012; Henry, et al., 2016) with very high internal consistency.

- The Major Depression Inventory (Bech \& Wermuth, 1998).

- The WHO-Quality of life questionnaire (WHO, 1998).

- Cima et al. (2011) proposed other questionnaires, such as

- The Tinnitus Catastrophizing Scale used for assessing cognitive misinterpretations of Tinnitus sounds and the Fear of Tinnitus Questionnaire for measuring tinnitus-related unpleasant emotions.

- HADS (Bjelland, et al., 2002) to evaluate negative emotional affects and to replace the Major Depression Inventory (MDI), which assess only depression. Moreover, stress is widely evaluated with the Perceived Stress Questionnaire (PSQ-30) (Levenstein, et al., 1993). The combination of HADS and PSQ-30 allows the distinct evaluation of stress, anxiety and depression.

- Hyperacusis Questionnaire is commonly used because about $40-55 \%$ of patients with Tinnitus experience this condition (Baguley, 2003; Schecklmann, et al., 2014). 
- The Health Utilities Index is validated for assessing quality of life of patients with tinnitus (Maes, et al., 2011; Frisch, 2014) which may substitute the WHO questionnaire (Zeman, et al., 2014).

- Other questionnaires proposed in the last years are

- tinnitus reaction questionnaire (Wilson, et al., 1991),

- insomnia severity index (Bastien, et al., 2001),

- clinical global impression-improvement (Kadouri, et al., 2007),

- tinnitus acceptance questionnaire (Weise, et al., 2013),

- Beck depression inventory (Beck, et al., 1996),

- tinnitus functional index (Henry, et al., 2016),

- health utilities index score (Horsman, et al., 2003),

- perceived stress questionnaire (Levenstein, et al., 1993),

- Some researchers opt for elaborating their own questionnaire-based evaluation such as Van de Heyning et al. (2014) who monitored patientreported outcomes and self-reporting of treatment (Van de Heyning, et al., 2014), and Spiegel et al. (2015) who applied a tinnitus severity numeric scale; a depression, anxiety and stress scale questionnaire; a comprehension attention battery; and auditory-visual multiprocessing tests (Spiegel, et al., 2015). 


\section{ANNEX N: ONLINE QUESTIONNAIRES}

The questionnaires are built online and with Google forms ${ }^{38}$. Some pictures of the information requested along 3 steps are shown below
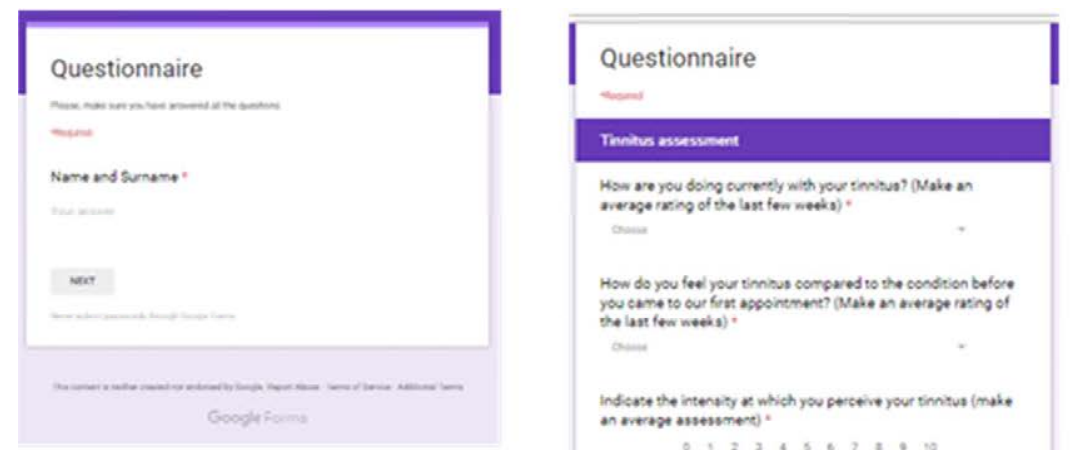

Questionnaire

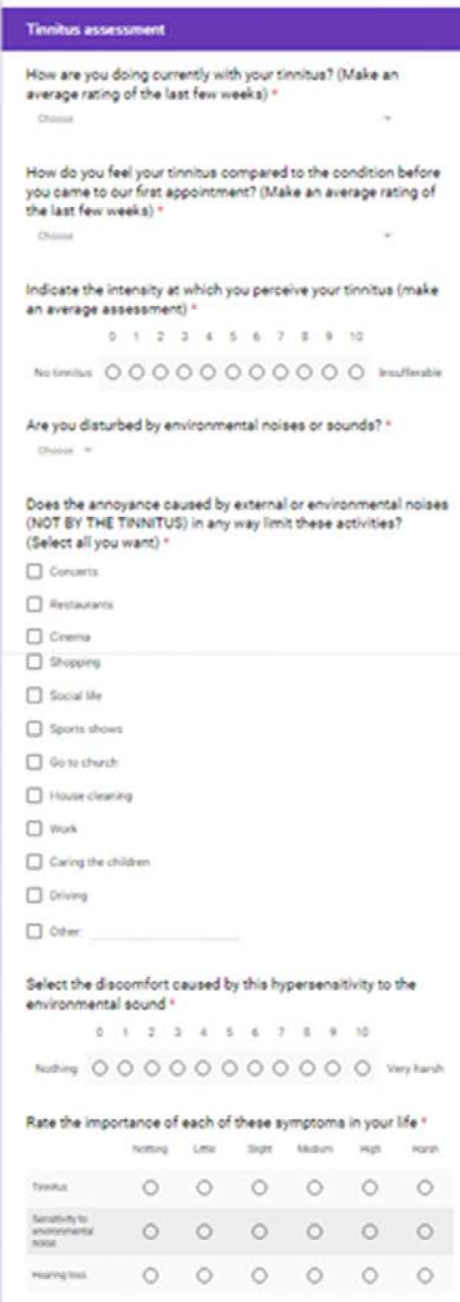

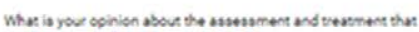

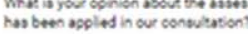

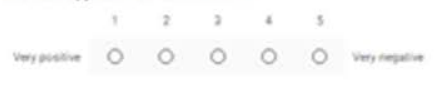

wat

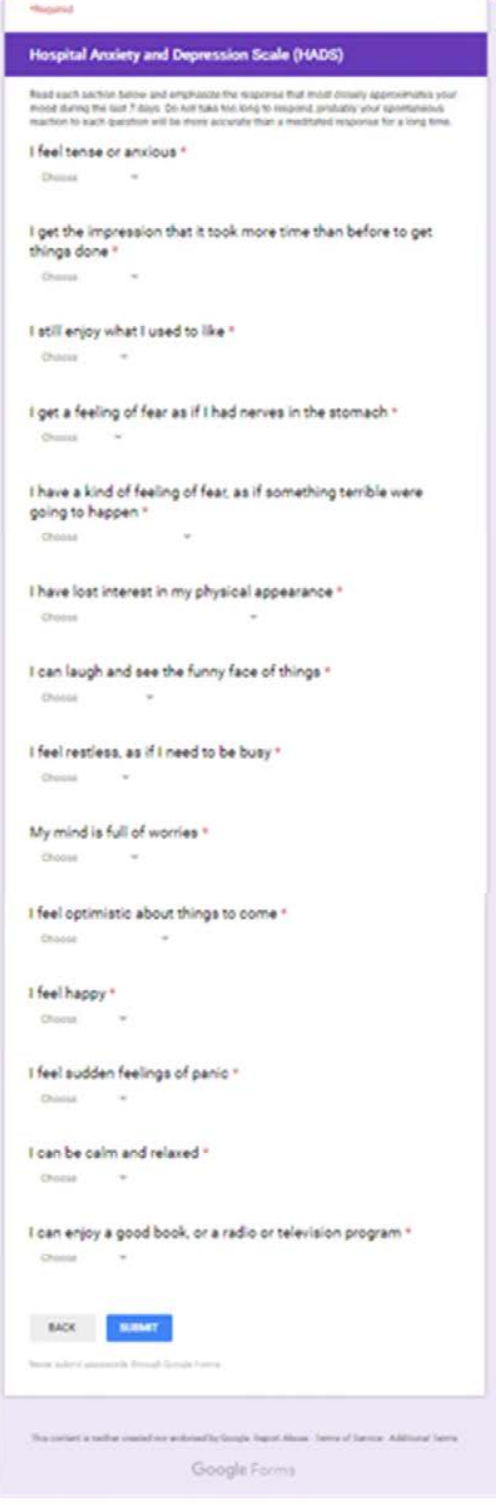

Figure 24: online questionnaires answered by patients through Google forms

\footnotetext{
${ }^{38}$ It can be accessed by this link https://doi.org/10.6084/m9.figshare.5558785.v1
} 


\section{ANNEX O: PROTOCOL APPROVAL DOCUMENTATION}

Tecnológico de Monterrey

Escuela de Medicina

Dra. Luz María Alonso Valerdi

Monterrey, N.L. a 20 de Junio del 2016 Investigadora Principal

Presente:

Estimada Dra. Alonso Valerdi:

Por medio de la presente le informamos a Usted que el Comité de Ética en Investigación y el Comité de Investigación, han revisado los documentos del Protocolo: AcústicaEEG

Titulado: "Evaluación Electroencefalográfica del Efecto de las Terapias Acústicas para el Tratamiento del Tinnitus Crónico y Refractario", que a continuación se enuncian:

- Protocolo de Investigación Titulado: "Evaluación Electroencefalográfica del Efecto de las Terapias Acústicas para el Tratamiento del Tinnitus Crónico y Refractario", Versión 2 , fechado 06 de mayo de 2016.

- Consentimiento Informado, Versión 2, fechado 06 de mayo de 2016.

Le informamos que en la sesión ordinaria del 20 de Junio del 2016 cumpliendo con los lineamientos de las GCP-ICH y leyes locales vigentes en México, teniendo un quórum de 4 miembros del Comité de Ética y 5 miembros del Comité de Investigación; los documentos mencionados con anterioridad han sido revisados en los aspectos técnicos y éticos por el Comité de Ética en Investigación de la Escuela de Medicina del Instituto Tecnológico y de Estudios Superiores de Monterrey, y el Comité de Investigación de la Escuela de Medicina del Instituto Tecnológico y de Estudios Superiores de Monterrey, con número de registro ante la Comisión Nacional de Bioética CONBIOETICA19CEI00820130520 y con registro ante COFEPRIS 13CEI19039139 (Comité de Etica) con número de registro ante COFEPRIS 13CI19039138 (Comité de Investigación), por lo que queda en carácter de:
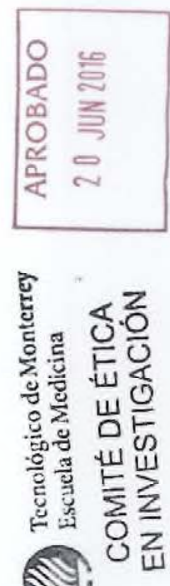

\section{Aprobado}

Sin más por el momento quedamos a sus órdenes.

Atentamente:

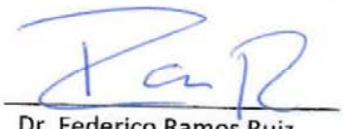

Presidente del

Comité de Ética en

Investigación

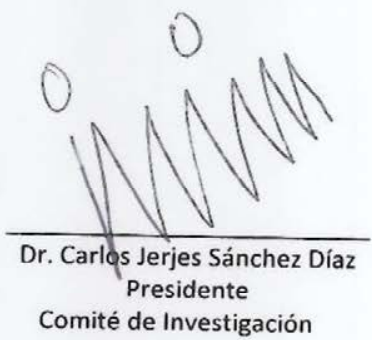

Comité de Investigación

Figure 25: Protocol used approved by TEC 
ANNEX P: PATIENT SCORE EVOLUTION FROM QUESTIONNAIRES
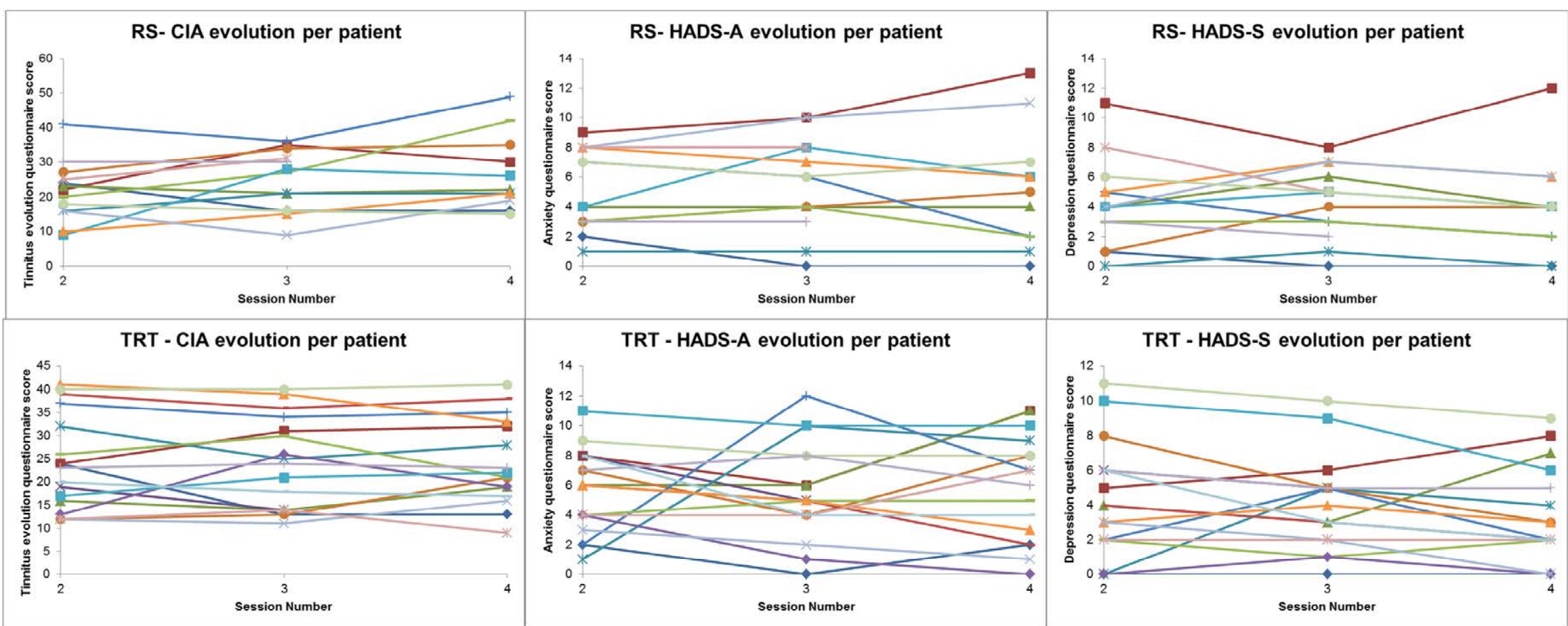

TRT - HADS-A evolution per patient

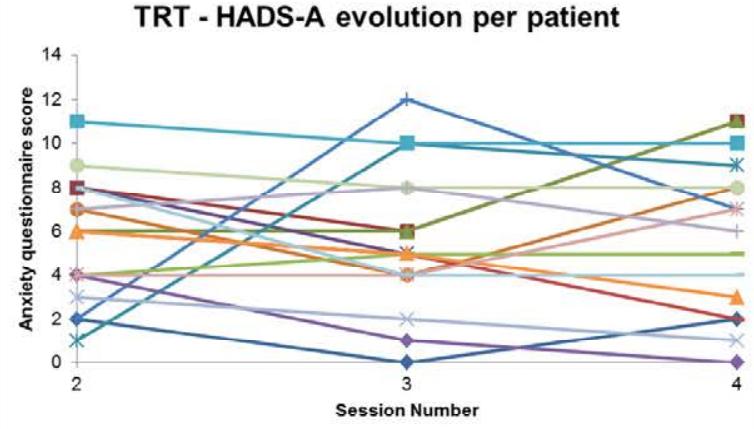

TRT - HADS-S evolution per patient

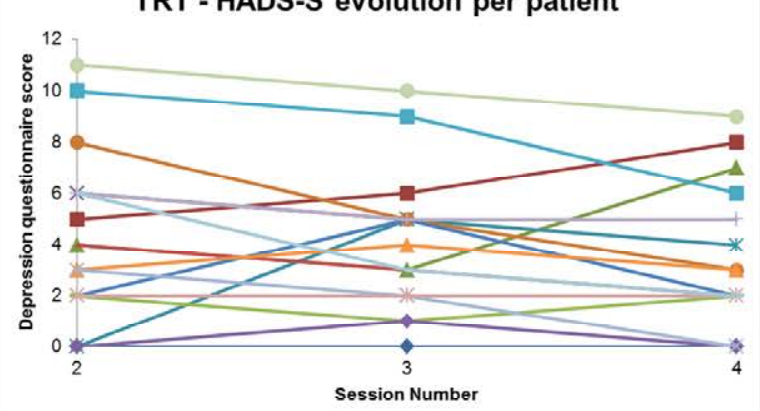

EAE - CIA evolution per patient

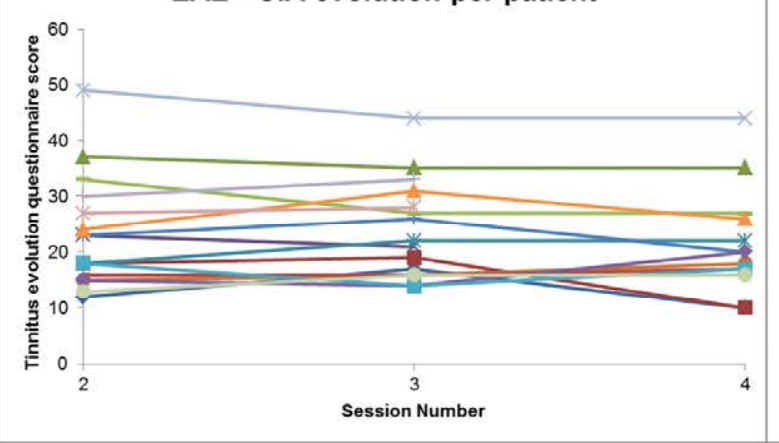

EAE - HADS-A evolution per patient
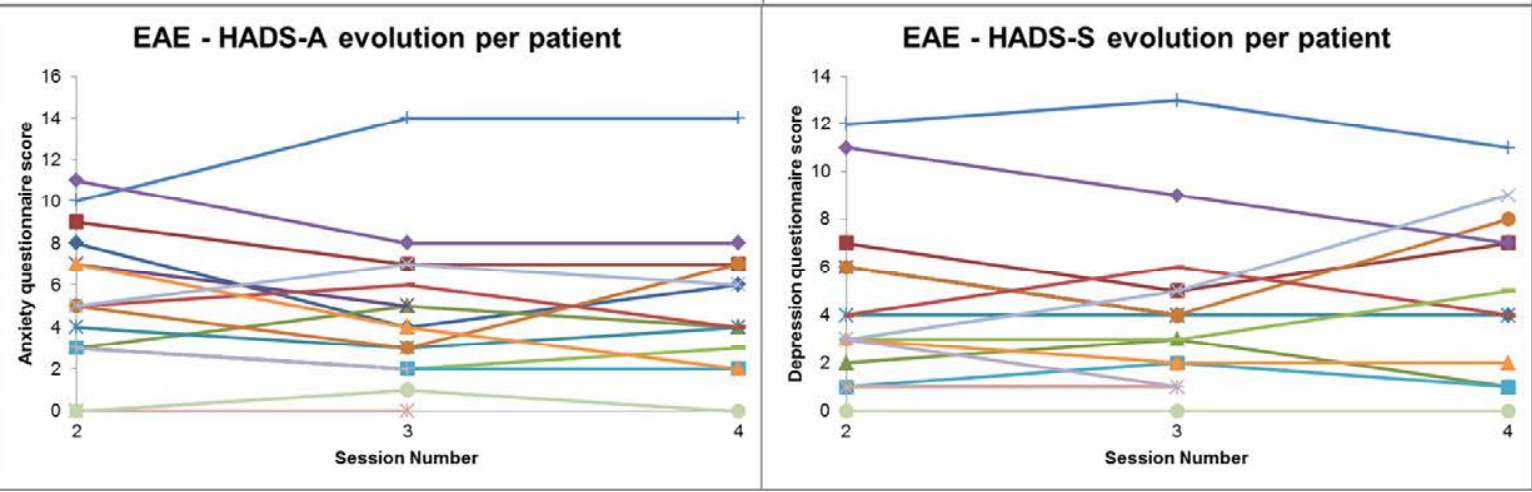


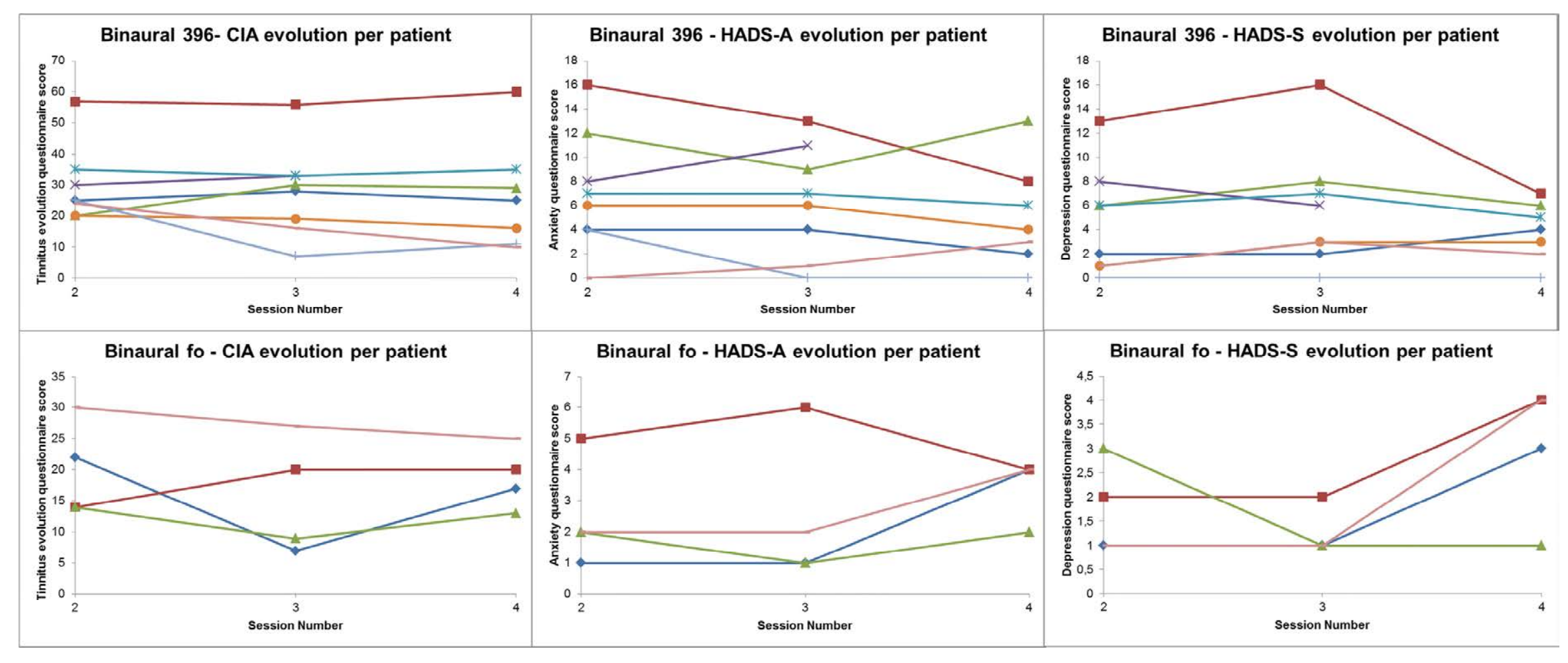

Figure 26: Subjective patient score representation 


\section{ANNEX Q: TINNITUS PERCEPTION EVOLUTION CONCLUSIONS ACCORDING TO THE DIFFERENT INITIAL CIA.EVO}

The next table explains the matrix of possibilities with the average and standard deviation calculation per therapy and CIA.EVO, and where "Non Applicable (N/A)" means there is no info.

\begin{tabular}{|c|c|c|c|c|c|c|c|c|c|c|c|c|c|c|c|}
\hline \multirow{2}{*}{$\begin{array}{c}\text { Initial } \\
\text { CIA }\end{array}$} & \multicolumn{3}{|c|}{$\mathrm{RS}$} & \multicolumn{3}{|c|}{ TRT } & \multicolumn{3}{|c|}{ EAE } & \multicolumn{3}{|c|}{ Binaural 396} & \multicolumn{3}{|c|}{ Binaural $\mathrm{f}_{\mathrm{o}}$} \\
\hline & $\mathrm{N}$ & $\bar{x}$ & $\sigma$ & $\mathrm{N}$ & $\bar{x}$ & $\sigma$ & $\mathrm{N}$ & $\bar{x}$ & $\sigma$ & $\mathrm{N}$ & $\bar{x}$ & $\sigma$ & $\mathrm{N}$ & $\bar{x}$ & $\sigma$ \\
\hline 1 & 2 & 1,50 & 0,71 & 0 & $\mathrm{~N} / \mathrm{A}$ & $\mathrm{N} / \mathrm{A}$ & 0 & $\mathrm{~N} / \mathrm{A}$ & $\mathrm{N} / \mathrm{A}$ & 0 & $\mathrm{~N} / \mathrm{A}$ & $\mathrm{N} / \mathrm{A}$ & 0 & $\mathrm{~N} / \mathrm{A}$ & $\mathrm{N} / \mathrm{A}$ \\
\hline 2 & 6 & 0,50 & 0,84 & 9 & $-0,11$ & 0,33 & 10 & $-0,20$ & 0,42 & 2 & 0,50 & 0,71 & 3 & 0 & 0 \\
\hline 3 & 4 & $-0,25$ & 0,50 & 4 & $-0,50$ & 0,58 & 4 & 0 & 0 & 5 & $-0,60$ & 0,89 & 1 & 0 & 0 \\
\hline 4 & 1 & 0 & 0 & 4 & $-0,50$ & 0,58 & 2 & $-0,50$ & 0,71 & 0 & $\mathrm{~N} / \mathrm{A}$ & $\mathrm{N} / \mathrm{A}$ & 0 & $\mathrm{~N} / \mathrm{A}$ & $\mathrm{N} / \mathrm{A}$ \\
\hline 5 & 0 & $\mathrm{~N} / \mathrm{A}$ & $\mathrm{N} / \mathrm{A}$ & 0 & $\mathrm{~N} / \mathrm{A}$ & $\mathrm{N} / \mathrm{A}$ & 0 & $\mathrm{~N} / \mathrm{A}$ & $\mathrm{N} / \mathrm{A}$ & 1 & 0 & 0 & 0 & $\mathrm{~N} / \mathrm{A}$ & $\mathrm{N} / \mathrm{A}$ \\
\hline
\end{tabular}

Table 10: Number of patients, average and standard deviation calculation from the evolution of Tinnitus perception scale per Therapy and Initial CIA.EVO.

Focusing in the CIA.EVO $=2$ and CIA.EVO $=3$ where there is data from all the signals, it can reflects that same 3 therapies commented previously have a better performance as

- $\quad$ CIA.EVO = 2: EAE and TRT (with a slightly better efficiency and success probability)

- CIA.EVO = 3: Binaural 396 Hz and TRT (with a bit increase of accuracy)

Same conclusions with other way to visualize it are drawn in the following graphics where it is shown the therapy performance evolution of tinnitus perception in the CIA.EVO 2 and 3.
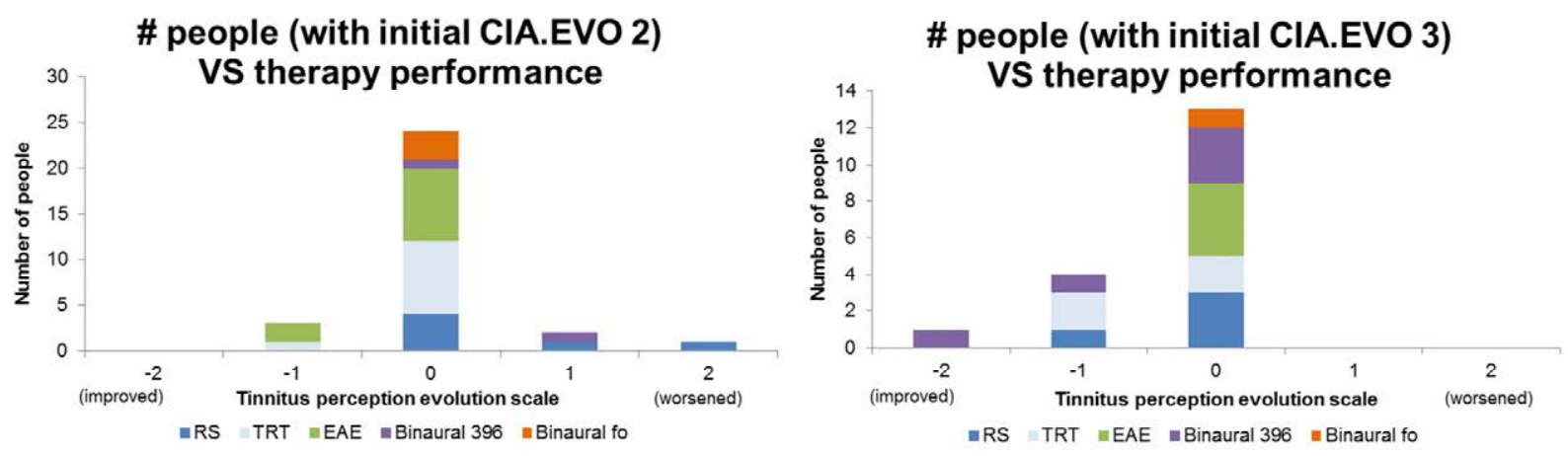

Figure 27: evolution of Tinnitus perception scale per Therapy at initial CIA.EVO 2 and 3 
ANNEX R: ANXIETY EVOLUTION ADDITIONAL DATA

\begin{tabular}{|c|c|c|}
\hline Therapy & $(\bar{x})$ & $\sigma$ \\
\hline RS & 0,08 & 0,49 \\
\hline TRT & 0,12 & 0,78 \\
\hline EAE & $-\mathbf{0 , 1 3}$ & $\mathbf{0 , 5 0}$ \\
\hline Binaural $396 \mathrm{~Hz}$ & 0 & 0,53 \\
\hline Binaural $\mathrm{f}_{\mathrm{o}}$ & 0 & 0 \\
\hline
\end{tabular}

Table 11: Average and standard deviation calculation from the evolution of Anxiety perception scale per Therapy

\begin{tabular}{|c|c|c|c|c|c|c|c|c|c|c|c|c|c|c|c|}
\hline \multirow{2}{*}{$\begin{array}{c}\text { Initial } \\
\text { HADS-A }\end{array}$} & \multicolumn{3}{|c|}{$\mathrm{RS}$} & \multicolumn{3}{|c|}{ TRT } & \multicolumn{3}{|c|}{ EAE } & \multicolumn{3}{|c|}{ Binaural 396} & \multicolumn{3}{|c|}{ Binaural $f_{o}$} \\
\hline & $\mathrm{N}$ & $\bar{x}$ & $\sigma$ & $\mathrm{N}$ & $\bar{x}$ & $\sigma$ & $\mathrm{N}$ & $\bar{x}$ & $\sigma$ & $\mathrm{N}$ & $\bar{x}$ & $\sigma$ & $N$ & $\bar{x}$ & $\sigma$ \\
\hline 1 & 9 & 0 & 0 & 12 & 0,33 & 0,65 & 12 & 0 & 0 & 5 & 0 & 0 & 4 & 0 & 0 \\
\hline 2 & 4 & 0,25 & 0,96 & 4 & $-0,25$ & 0,96 & 3 & $-0,33$ & 1,15 & 1 & 1 & 0 & 0 & $\mathrm{~N} / \mathrm{A}$ & $\mathrm{N} / \mathrm{A}$ \\
\hline 3 & 0 & $\mathrm{~N} / \mathrm{A}$ & $\mathrm{N} / \mathrm{A}$ & 1 & -1 & 0 & 1 & -1 & 0 & 2 & $-0,5$ & 0,71 & 0 & $\mathrm{~N} / \mathrm{A}$ & $\mathrm{N} / \mathrm{A}$ \\
\hline
\end{tabular}

Table 12: Number of patients, average and standard deviation calculation from the evolution of Anxiety scale per Therapy and Initial HADS-A. 
ANNEX S: DEPRESSION EVOLUTION ADDITIONAL DATA

\begin{tabular}{|c|c|c|}
\hline Therapy & $(\bar{x})$ & $\sigma$ \\
\hline RS & $-0,08$ & 0,28 \\
\hline TRT & $-0,12$ & 0,49 \\
\hline EAE & 0 & 0,63 \\
\hline Binaural $396 \mathrm{~Hz}$ & $-\mathbf{0 , 5 0}$ & $\mathbf{0 , 7 6}$ \\
\hline Binaural $\mathrm{f}_{\mathrm{o}}$ & 0 & 0 \\
\hline
\end{tabular}

Table 13: Average and standard deviation calculation from the evolution of Depression scale per Therapy

\begin{tabular}{|c|c|c|c|c|c|c|c|c|c|c|c|c|c|c|c|}
\hline \multirow{2}{*}{$\begin{array}{c}\text { Initial } \\
\text { HADS-D }\end{array}$} & \multicolumn{3}{|c|}{ RS } & \multicolumn{3}{|c|}{ TRT } & \multicolumn{3}{|c|}{ EAE } & \multicolumn{3}{|c|}{ Binaural 396} & \multicolumn{3}{|c|}{ Binaural $f_{o}$} \\
\hline & $\mathrm{N}$ & $\bar{x}$ & $\sigma$ & $\mathrm{N}$ & $\bar{x}$ & $\sigma$ & $\mathrm{N}$ & $\bar{x}$ & $\sigma$ & $\mathrm{N}$ & $\bar{x}$ & $\sigma$ & $\mathrm{N}$ & $\bar{x}$ & $\sigma$ \\
\hline 1 & 11 & 0 & 0 & 14 & 0,07 & 0,27 & 14 & 0,14 & 0,36 & 5 & 0 & 0 & 4 & 0 & 0 \\
\hline 2 & 1 & -1 & 0 & 2 & -1 & 0 & 0 & $\mathrm{~N} / \mathrm{A}$ & $\mathrm{N} / \mathrm{A}$ & 2 & -1 & 0 & 0 & $\mathrm{~N} / \mathrm{A}$ & $\mathrm{N} / \mathrm{A}$ \\
\hline 3 & 1 & 0 & 0 & 1 & -1 & 0 & 2 & -1 & 1,41 & 1 & -2 & 0 & 0 & $\mathrm{~N} / \mathrm{A}$ & $\mathrm{N} / \mathrm{A}$ \\
\hline
\end{tabular}

Table 14: Number of patients, average and standard deviation calculation from the evolution of Depression scale per Therapy and Initial HADS-D. 


\section{ANNEX T: COMMON STATISTICAL FEATURES IN THE TIME DOMAIN ${ }^{39,40}$}

- Mean: average of the amplitude values over sample length of the signal. The mean is a good approximation of the central tendency for unimodal symmetric distributions but can be misleading in skewed or multimodal distributions.

$$
\bar{X}=\frac{1}{N} \sum_{n=1}^{N} X_{n}
$$

- Mean absolute value (MAV): Estimates the mean absolute value of each segment by adding the absolute value of all the values $x_{i}$-ith point, the current point of signal $x$, and dividing it by the length of the segment.

$$
M A V_{k}=\frac{1}{N} \sum_{n=1}^{N}\left|x_{n}\right|
$$

- Median: A sample value separating higher from lower half of a dataset.

$$
\text { Med }= \begin{cases}\frac{X_{\frac{N}{2}}+X_{\frac{N}{2}+1}}{2} & \text { for even } N \\ \frac{X_{N+1}}{2} & \text { for odd } N\end{cases}
$$

- Maximum Value (Max): The maximum instantaneous value of the signal as measured from the zero level. $\quad X_{\max }=\max \left(x_{n}\right)$

- Minimum Value (min): The minimum instantaneous value of the signal as measured from the zero level. $\quad X_{\min }=\min \left(x_{n}\right)$

- Peak: The maximum instantaneous absolute value of the signal as measured from the zero level.

$$
X_{\text {peak }}=\max \left|x_{n}\right|
$$

- Simple square integral (SSI): It calculates the energy of the EEG signal according to

$$
S S I_{k}=\sum_{n=1}^{N}\left|x_{n}^{2}\right|
$$

- Integrated EEG (IEEG): It computes the summation of the absolute values of EEG signals

$$
I E E G_{k}=\sum_{n=1}^{N}\left|x_{n}\right|
$$

- Waveform length (WL): It is the cumulative length of the waveform over the segment. It indicates a measure of waveform amplitude, frequency and duration, all within a single parameter. $\quad W L_{k}=\sum_{n=1}^{N-1}\left|x_{n+1}-x_{n}\right|$

- Modified mean absolute value1 (MMAV1): It is an extension of MAV using the define weighting function $\mathrm{w}_{\mathrm{n}}$

$$
\begin{gathered}
\text { MMAV } 1_{k}=\frac{1}{N} \sum_{n=1}^{N} w_{n}\left|x_{n}\right| \\
w(n)= \begin{cases}1, & 0.25 N \leq n \leq 0.75 N \\
0.5, & \text { otherwise }\end{cases}
\end{gathered}
$$

- Modified mean absolute value2 (MMAV2): It has a continuous weighting function of $w_{n}$. This function is known as an improvement to the modified version of MAV.

\footnotetext{
${ }^{39}$ In all the equations, $\boldsymbol{N}$ is the length of the segment, $\boldsymbol{k}$ is the current segment, $\boldsymbol{x}_{\boldsymbol{i}}$ is the current point of signal and $\boldsymbol{n}$ is the index of the current point.

${ }_{40}$ (Khorshidtalab, et al., 2013; Lei, 2017; Boostani, et al., 2017; Altın \& Er, 2016; Englehart \& Hudgins, 2003; Bronzino, 1995; Phinyomark, et al., 2009; Huang \& Chen, 1999; Oskoei \& Hu, 2006; RechyRamirez \& Hu, 2011)
} 


$$
\begin{gathered}
M M A V 2_{k}=\frac{1}{N} \sum_{n=1}^{N} w_{n}\left|x_{n}\right| \\
w(n)= \begin{cases}1, \quad 0.25 N \leq n \leq 0.75 N \\
\frac{4 n}{N}, \quad 0.25 N>n \\
\frac{4(n-N)}{N}, & 0.75 N<n\end{cases}
\end{gathered}
$$

- Standard Deviation: A measure that is used to quantify the amount of variation or dispersion of a set of data values. A low standard deviation indicates that the data points tend to be close to the mean (also called the expected value) of the set, while a high standard deviation indicates that the data points are spread out over a wider range of values. $\quad S T D_{k}=\sqrt{\frac{\sum_{n=1}^{N}\left(X_{n}-\bar{X}\right)^{2}}{N-1}}$

- Variance: A measure of how far a set of numbers is spread out from mean.

$$
V A R_{k}=\frac{\sum_{n=1}^{N}\left(X_{n}-\bar{X}\right)^{2}}{N-1}=\left(S T D_{k}\right)^{2}
$$

- Root: It is a measure of the energy content of a signal.

$$
X_{\text {root }}=\left(\frac{\sum_{n=1}^{N} \sqrt{\left|x_{n}\right|}}{N}\right)^{2}
$$

- Root mean square (RMS): It is a common, widely used feature for variety of biosignals. Known as the quadratic mean, It is defined as the squared root of the mean square.

$$
X_{R M S}=\sqrt{\frac{\sum_{n=1}^{N}\left(X_{n}\right)^{2}}{N}}
$$

- Crest: Measure of a waveform, showing the ratio of peak values to the effective value. In other words, crest factor indicates how extreme the peaks are in a waveform. It reflects the intensity degree of the oscillation. Crest factor 1 indicates no peaks.

$$
\text { Crest }_{k}=\frac{X_{\text {peak }}}{X_{R M S}}
$$

- Skewness: This parameter measures the degree of deviation from the symmetry of anormal distribution. This measure has the value of zero when the distribution is completely symmetrical or some nonzero values when the EEG distribution is asymmetrical, with respect to the baseline. For a unimodal distribution, negative skew indicates that the tail on the left side of the probability density function is longer or fatter than the right side - it does not distinguish these two kinds of shape. Conversely, positive skew indicates that the tail on the right side is longer or fatter than the left side.

$$
\text { Skewness }_{k}=\frac{\sum_{n=1}^{N} \frac{\left(X_{n}-\bar{X}\right)^{3}}{N}}{\left[\sum_{n=1}^{N} \frac{\left(x_{n}-\bar{X}\right)^{2}}{N}\right]^{3 / 2}}
$$

- Kurtosis: Describes the 'peakedness' in a curve and the thickness of the tails. It does not affect the central tendency and occurs in normally distributed data sets. 
- Leptokurtic: Tall and skinny compared with an excess of extreme values causing the tails to be thicker than the Normal bell curve $(>0)$

- Platykurtic: Short and fat with fewer extreme values causing the tails to be thinner than the Normal bell curve $(<0)$

- Mesokurtic: Normal bell curve $(=0)$.

$$
\text { Kurtosis }_{k}=\frac{\sum_{n=1}^{N} \frac{\left(X_{n}-\bar{x}\right)^{4}}{N}}{\sum_{n=1}^{N}\left[\frac{\left[X_{n}-\bar{X}\right)}{N}\right]^{2}}
$$

- Clearance: It refers to the difference between the maximum amplitude (peak) and RMS amplitude.

$$
\text { Clearance }_{k}=\frac{X_{\text {peak }}}{X_{\text {root }}}
$$

- Shape: It described by the following characteristics: symmetry, number of peaks, skewness and uniformity. $\quad$ Shape $_{k}=\frac{X_{R M S}}{\frac{1}{N} \sum_{n=1}^{N}\left|x_{n}\right|}$

- Impulse: the output of a dynamic system when presented with a brief input signal, called an impulse. More generally, an impulse response is the reaction of any dynamic system in response to some external change. In both cases, the impulse describes the reaction of the system as a function of time (or possibly as a function of some other independent variable that parameterizes the dynamic behaviour of the system).

$$
\text { Impulse }_{k}=\frac{X_{\text {peak }}}{\frac{1}{N} \sum_{n=1}^{N}\left|x_{n}\right|}
$$




\section{ANNEX U: COMMON STATISTICAL FEATURES IN THE FREQUENCY DOMAIN ${ }^{41,42}$}

- Power Spectra Density (PSD): It refers to the spectral energy distribution that would be found per unit time, since the total energy of such a signal over all time would generally be infinite. Summation or integration of the spectral components yields the total power, identical to what would be obtained by integrating $x^{2}(t)$ over the time domain, as dictated by Parseval's theorem (Stoica \& Moses, 2005).

$$
E=\int_{-\infty}^{\infty}|x(t)|^{2} d t=\int_{-\infty}^{\infty}|\hat{x}(f)|^{2} d f \quad \text { where } \quad \hat{x}(f)=\int_{-\infty}^{\infty} e^{-2 \pi i f t} x(t) d t
$$

- Common statistical features in the frequency domain when a fault occurs in a machinery:

- Feature $\mathrm{P} 1$, namely mean frequency $\left(\mathrm{X}_{\mathrm{mf}}\right)$, may indicate the vibration energy in the frequency domain, which represents the average of the amplitudes of all the frequencies.

- Features P2 - P4, P6, and P11 - P13 may describe the convergence of the spectrum power, reflecting the energy of frequency spectrum.

- Features P5 (MNF or $\left.\mathrm{X}_{\mathrm{fc}}\right)$ and P7 $\left(\mathrm{X}_{\mathrm{rmsf}}\right)$ - P9 may show the position change of main frequencies which are dominant in the frequency spectrum.

$$
\begin{aligned}
& P 1=\frac{\sum_{k=1}^{K} s(k)}{K} \quad \text { (34), } \quad P 2=\frac{\sum_{k=1}^{K}\left(s(k)-P_{1}\right)^{2}}{K-1}(35), \quad P 3=\frac{\sum_{k=1}^{K}\left(s(k)-P_{1}\right)^{3}}{K\left(\sqrt{P_{2}}\right)^{3}} \\
& P 4=\frac{\sum_{k=1}^{K}\left(s(k)-P_{1}\right)^{4}}{K P_{2}^{2}}(37), \quad P 5=\frac{\sum_{k=1}^{K} f_{k} s(k)}{\sum_{k=1}^{K} s(k)} \quad \text { (38), } \quad P 6=\sqrt{\frac{\sum_{k=1}^{K}\left(f_{k}-P_{5}\right)^{2} s(k)}{K}} \\
& P 7=\sqrt{\frac{\sum_{k=1}^{K} f_{k}^{2} s(k)}{\sum_{k=1}^{K} s(k)}} \quad(40), \quad P 8=\sqrt{\frac{\sum_{k=1}^{K} f_{k}^{4} s(k)}{\sum_{k=1}^{K} f_{k}^{2} s(k)}} \quad(41), \quad P 9=\frac{\sum_{k=1}^{K} f_{k}^{2} s(k)}{\sqrt{\sum_{k=1}^{K} s(k) \sum_{k=1}^{K} f_{k}^{4} s(k)}} \\
& P 10=\frac{P_{6}}{P_{5}} \quad(43), \quad P 11=\frac{\sum_{k=1}^{K}\left(f_{k}-P_{5}\right)^{3} s(k)}{K P_{6}^{3}}(44), \quad P 12=\frac{\sum_{k=1}^{K}\left(f_{k}-P_{5}\right)^{4} s(k)}{K P_{6}^{4}} \\
& P 13=\frac{\sum_{k=1}^{K} \sqrt{\left(f_{k}-P_{5}\right)} s(k)}{K \sqrt{P_{6}}} \quad(46), \quad P 14=\sqrt{\frac{\sum_{k=1}^{K}\left(f_{k}-P_{5}\right)^{2} s(k)}{\sum_{k=1}^{K} s(k)}}
\end{aligned}
$$

- Median frequency (MDF): It is a frequency at which the spectrum is divided into two regions with equal amplitude.

$$
\sum_{k=1}^{M D F} s(k)=\sum_{k=M D F}^{K} s(k)=\frac{1}{2} \sum_{k=1}^{K} s(k)
$$

- Power Spectrum Deformation ratio: It is sensitive to changes in spectral symmetry

and provides an indication of spectral deformation. $\quad \Omega=\frac{\sqrt{\frac{M_{2}}{M_{0}}}}{\frac{M_{1}}{M_{0}}}$

$M_{n}$ is the $n^{\text {th }}$ spectral moment defined as:

$$
M_{n}=\sum_{k=1}^{K} s(k) f_{k}^{n}
$$

\footnotetext{
${ }^{41}$ where $\boldsymbol{s}(\boldsymbol{k})$ is a spectrum for $\boldsymbol{k}=1,2, \ldots, \mathrm{K}, \boldsymbol{K}$ is the number of spectrum lines and $\boldsymbol{f}_{\boldsymbol{k}}$ is the frequency value of the $k^{\text {th }}$ spectrum line.

${ }^{42}$ (Lei, 2017; Si, et al., 2017; Altın \& Er, 2016)
} 


\section{ANNEX V: WICA ALGORITHM FOR ARTIFACT SUPPRESSION IN EEG}

ICA starts assuming that $\mathrm{K}$ simultaneously recorded $\mathrm{EEG}$ signals $\mathrm{X}(\mathrm{t})=\left\{\mathrm{X}_{1}(\mathrm{t}), \ldots, \mathrm{X}_{\mathrm{K}}(\mathrm{t})\right\}$ are linear mixtures of $N(N \leq K)$ a priori unknown independent components (sources) $S(t)$ $=\left\{\mathrm{S}_{1}(\mathrm{t}), \ldots, \mathrm{S}_{\mathrm{N}}(\mathrm{t})\right\}$ including artifactual and of the neural origin:

where

$$
X(t)=M \cdot S(t)
$$

$M$ is the unknown mixing matrix defining weights at which each source is present in the EEG signals recorded at the scalp.

$\mathrm{S}$ is an independent component split into a high amplitude artifact $\mathrm{a}(\mathrm{t})$ and a low amplitude residual neural signal $n(t)$ :

$$
S_{i}(t)=a_{i}(t)+n_{i}(t)
$$

The continuous wavelet transform (WT) of the independent component $\mathrm{s}_{\mathrm{i}}(\mathrm{t})$ (somehow similar to the Fourier transform) reads:

where

$$
W^{S}(d, b)=\frac{1}{\sqrt{d}} \int s_{1}(t) \psi_{d, b}(t) d t, \psi_{d, b}=\psi\left(\frac{t-b}{d}\right)
$$

$W^{\mathcal{S}}(d, b)$ is the WT of $s_{1}(t), \psi$ is the mother wavelet with $b$ and $d$ defining the time localization and scale. The WT (14) is redundant and therefore in practice its discrete counterpart (DWT) is usually used. In the DWT the time scales and localizations take only fixed values, usually $d=2^{j}, b=k 2^{j}$ with integers $j$ and $k$ playing roles of the decomposition level and temporal localization at this level, respectively. Using Equations (13) and (14) it can be written:

where

$$
W^{\mathcal{s}}(d, b)=W^{a}(d, b)+W^{n}(d, b)
$$

$W^{a}(d, b)$ and $W^{n}(d, b)$ are the wavelet coefficient obtained by the transformation (14) of the artificial and neural parts of the component, respectively.

wICA algorithm can be explained in the following steps (Castellanos \& Makarov, 2006):

1. Apply a conventional ICA decomposition to raw EEG thus obtaining the mixing matrix $\mathrm{M}$ and $\mathrm{N}$ independent components $\left\{s_{1}(t), s_{2}(t), \ldots, s_{N}(t)\right\}$.

2. Wavelet transform components obtaining their representations $\{W(j, k)\} s_{j}$.

3. Threshold the wavelet coefficients, i.e. set $W(j, k)=0$ for those that are higher than the threshold, $|W(j, k)|>K$.

4. Inverse wavelet transform of the thresholded coefficients $W(j, k)$ thus recomposing components consisting sources of the neural origin only $\left\{n_{i}(t)\right\}$.

5. Compose wICA-corrected EEG: $\tilde{X}(t)=M \cdot\left[n_{1}(t), n_{2}(t), \ldots, n_{N}(t)\right]^{T}$.

Selection of the threshold value, $\mathrm{K}$, is an essential element of the algorithm. Here it has been used the simplest fixed form threshold:

where

$$
K=\sqrt{2 \log N} \sigma
$$

$N$ is the length of the data segment to be processed, and

$\sigma^{2}=\operatorname{median}(|W(d, b)|) / 0.6745$ is the estimator of the magnitude of the neural wide band signal part. 


\section{ANNEX W: SESSION 4 - SESSION 1 DIFFERENCE OF TIME AND TREQUENCY DOMAIN PARAMETERS FROM EACH ACOUSTIC THERAPY FOR EO}

In the following tables are found the Average $(\mu)$ and Standard Deviation $(\sigma)$ of the time and frequency domain parameters difference between session 4 and session 1 (relativized by Session 1) for the resting condition (Eyes Open) of all patients per acoustic therapy.

- Binaural $f_{0}$ therapy (data from 2 patients)

Frequency domain parameters

\begin{tabular}{|c|c|c|c|c|c|c|c|c|c|c|c|c|c|c|c|c|c|c|c|c|c|c|}
\hline Channel & PSDtotal & PSD- $\delta$ & PSD- $\theta$ & PSD- $\alpha$ & PSD- $\beta$ & PSD $-\gamma$ & $\mathrm{P} 1(\mathrm{Xmf})$ & P2 & P3 & P4 & P5(Xfc) & P6 & P7(Xrmsf) & P8 & P9 & P10 & P11 & P12 & P13 & P14 & MDF & PSDef \\
\hline \begin{tabular}{l|l|}
1 & $\mu$ \\
\end{tabular} & 3.76 & 45.78 & 1.67 & 0.52 & 0.34 & 0.35 & 0.03 & 6.73 & 4.79 & 50.63 & \begin{tabular}{l|}
-0.16 \\
\end{tabular} & 0.10 & 0.08 & 0.02 & 0.10 & 0.35 & 0.74 & 0.00 & 0.04 & 0.08 & 0.27 & 0.10 \\
\hline 10 & 5.02 & 64.40 & 2.07 & 0.24 & 0.23 & 0.21 & 0.11 & 9.48 & 6.66 & 71.41 & 0.17 & 0.17 & 0.08 & 0.02 & 0.10 & 0.47 & 0.63 & 0.14 & 0.11 & 0.11 & 0.24 & 0.12 \\
\hline$\mu$ & 8.96 & 57.60 & 4.10 & 1.06 & 0.46 & 0.58 & 0.11 & 14.89 & 8.25 & 90.24 & 0.41 & 0.07 & 0.25 & 0.00 & 0.26 & 0.84 & 2.56 & 0.35 & 0.09 & 0.02 & 0.75 & 0.28 \\
\hline 20 & 2.14 & 51.03 & 1.87 & 0.05 & 0.30 & 0.28 & 0.17 & 4.39 & 3.78 & 65.00 & 0.08 & 0.04 & 0.05 & 0.01 & 0.06 & 0.19 & 0.81 & 0.23 & 0.12 & 0.04 & 0.08 & 0.09 \\
\hline$\mu$ & 5.21 & 28.86 & 1.16 & 0.43 & 0.49 & 0.61 & 0.11 & 10.46 & 2.15 & 5.18 & 0.22 & 0.13 & 0.12 & 0.04 & 0.15 & 0.57 & 6.65 & 0.11 & 0.09 & 0.08 & 0.39 & 0.17 \\
\hline$\sigma$ & 6.49 & 38.69 & 0.35 & 0.18 & 0.38 & 0.21 & 0.12 & 14.04 & 2.53 & 6.07 & 0.30 & 0.01 & 0.18 & 0.01 & 0.19 & 0.60 & 8.75 & 0.34 & 0.07 & 0.05 & 0.57 & 0.22 \\
\hline$\mu$ & 3.64 & 32.57 & 1.22 & 0.44 & 0.40 & 0.54 & 0.03 & 5.20 & 6.64 & 65.00 & 0.30 & 0.05 & 0.17 & 0.03 & 0.19 & 0.50 & 2.39 & 0.38 & 0.02 & 0.07 & 0.52 & 0.19 \\
\hline$4 \longdiv { \sigma }$ & 0.16 & 20.47 & 0.60 & 0.03 & 0.26 & 0.40 & 0.27 & 0.81 & 2.89 & 42.83 & 0.14 & 0.15 & 0.09 & 0.01 & 0.08 & 0.08 & 1.86 & 0.52 & 0.21 & 0.00 & 0.19 & 0.10 \\
\hline$\mu$ & 0.88 & 3.31 & 0.49 & 0.38 & 0.28 & 0.44 & 0.11 & 0.80 & 2.80 & 24.00 & 0.14 & 0.02 & 0.07 & 0.02 & 0.09 & 0.14 & 0.80 & 0.27 & 0.08 & 0.04 & 0.28 & 0.08 \\
\hline$\sigma$ & 0.68 & 3.65 & 0.35 & 0.18 & 0.07 & 0.23 & 0.08 & 0.83 & 3.18 & 31.97 & 0.08 & 0.03 & 0.04 & 0.01 & 0.04 & 0.07 & 0.05 & 0.11 & 0.05 & 0.01 & 0.15 & 0.05 \\
\hline$\mu$ & 4.84 & 33.88 & 0.76 & 0.46 & 0.38 & 0.50 & 0.02 & 9.55 & 7.20 & 107.87 & 0.23 & 0.06 & 0.12 & 0.04 & 0.16 & 0.43 & 1.52 & 0.25 & 0.01 & 0.07 & 0.40 & 0.16 \\
\hline$\sigma$ & 6.23 & 46.40 & 0.10 & 0.19 & 0.16 & 0.27 & 0.05 & 13.30 & 9.21 & 150.00 & 0.21 & 0.02 & 0.13 & 0.02 & 0.11 & 0.43 & 0.01 & 0.10 & 0.04 & 0.00 & 0.35 & 0.15 \\
\hline$\mu$ & 19.79 & 124.75 & 4.76 & 1.09 & 0.46 & 0.59 & 0.32 & 37.94 & 8.29 & 89.12 & 0.47 & 0.20 & 0.28 & 0.04 & 0.31 & 1.28 & 6.04 & 0.25 & 0.23 & 0.05 & 0.83 & 0.36 \\
\hline$\sigma$ & 2.41 & 106.93 & 5.09 & 0.57 & 0.09 & 0.07 & 0.04 & 0.08 & 4.28 & 95.44 & 0.02 & 0.12 & 0.01 & 0.01 & 0.02 & 0.29 & 11.32 & 0.21 & 0.05 & 0.09 & 0.04 & 0.02 \\
\hline$\mu$ & 24.26 & 147.15 & 1.83 & 0.89 & 0.58 & 0.57 & 0.37 & 61.30 & 7.27 & 55.07 & 0.38 & 0.30 & 0.21 & 0.06 & 0.25 & 1.20 & 4.20 & 0.05 & 0.26 & 0.15 & 0.64 & 0.29 \\
\hline$\sigma$ & 31.44 & 186.64 & 0.02 & 0.53 & 0.11 & 0.26 & 0.88 & 82.63 & 0.36 & 17.39 & 0.13 & 0.38 & 0.07 & 0.04 & 0.10 & 1.07 & 12.49 & 0.32 & 0.61 & 0.06 & 0.32 & 0.15 \\
\hline$\mu$ & 4.15 & 43.02 & 0.63 & 0.47 & 0.38 & 0.39 & 0.01 & 6.65 & 3.91 & 40.61 & 0.15 & 0.06 & 0.07 & 0.03 & 0.10 & 0.31 & 0.53 & 0.12 & 0.01 & 0.06 & 0.29 & 0.10 \\
\hline$\sigma$ & 5.10 & 59.73 & 0.59 & 0.44 & 0.08 & 0.09 & 0.27 & 10.09 & 6.01 & 58.18 & 0.20 & 0.22 & 0.10 & 0.02 & 0.11 & 0.57 & 0.25 & 0.24 & 0.22 & 0.08 & 0.37 & 0.14 \\
\hline$\mu$ & 1.09 & 9.12 & 0.26 & 0.20 & 0.26 & 0.40 & 0.10 & 0.97 & 2.26 & 21.19 & 0.07 & 0.01 & 0.03 & 0.04 & 0.06 & 0.09 & 0.47 & 0.22 & 0.07 & 0.04 & 0.16 & 0.05 \\
\hline$1 0 \longdiv { \sigma }$ & 0.92 & 11.78 & 0.06 & 0.04 & 0.06 & 0.11 & 0.11 & 2.07 & 3.84 & 31.11 & 0.18 & 0.08 & 0.11 & 0.03 & 0.08 & 0.30 & 0.10 & 0.08 & 0.09 & 0.02 & 0.31 & 0.09 \\
\hline \begin{tabular}{l|l} 
& $\mu$ \\
\end{tabular} & 8.88 & 41.65 & 2.25 & 0.93 & 0.31 & 0.50 & 0.31 & 17.67 & 6.73 & 61.28 & 0.33 & 0.27 & 0.17 & 0.07 & 0.23 & 0.89 & 4.68 & 0.08 & 0.23 & 0.11 & 0.56 & 0.24 \\
\hline$1 1 \longdiv { \sigma }$ & 3.68 & 1.22 & 1.37 & 0.07 & 0.18 & 0.19 & 0.12 & $\begin{array}{l}2.96 \\
\end{array}$ & 3.45 & 64.16 & 0.11 & 0.19 & 0.09 & 0.02 & 0.07 & 0.03 & 3.04 & 0.43 & 0.11 & 0.12 & 0.21 & 0.07 \\
\hline \begin{tabular}{l|l}
12 & $\mu$ \\
\end{tabular} & 14.71 & 26.37 & 1.47 & 0.70 & 0.44 & 0.47 & 0.27 & 22.71 & 2.03 & 6.21 & 0.27 & 0.18 & 0.14 & 0.03 & 0.17 & 0.64 & 4.08 & 0.05 & 0.19 & 0.07 & 0.49 & 0.17 \\
\hline$1 2 \longdiv { \sigma }$ & 18.80 & 35.36 & 0.68 & 0.38 & 0.10 & 0.28 & 0.70 & 29.98 & 1.81 & 7.44 & 0.07 & 0.30 & 0.03 & 0.01 & 0.04 & 0.58 & 7.23 & 0.38 & 0.49 & 0.04 & 0.26 & 0.07 \\
\hline \begin{tabular}{l|l}
$\mu$ \\
13
\end{tabular} & 1.70 & 2.71 & 0.38 & 0.51 & 0.90 & 0.26 & 0.02 & 2.71 & 3.02 & 24.50 & 0.12 & 0.01 & 0.06 & 0.00 & 0.06 & 0.17 & 0.19 & 0.09 & 0.01 & 0.01 & 0.18 & 0.06 \\
\hline$1 3 \longdiv { \sigma }$ & 1.98 & 3.52 & 0.03 & 0.02 & 0.94 & 0.01 & 0.21 & 3.51 & 3.73 & 33.67 & 0.13 & 0.10 & 0.08 & 0.01 & 0.08 & 0.28 & 0.23 & 0.04 & 0.15 & 0.01 & 0.25 & 0.07 \\
\hline \begin{tabular}{l|l}
$14 \mu$ \\
14
\end{tabular} & 29.10 & 247.34 & 5.24 & 2.27 & 0.90 & 0.28 & 0.92 & 54.35 & 4.28 & 26.28 & 0.37 & 0.43 & 0.21 & 0.06 & 0.26 & 1.36 & 2.14 & 0.13 & 0.59 & 0.04 & 0.60 & 0.27 \\
\hline$1 4 \longdiv { \sigma }$ & 20.56 & 308.17 & 4.53 & 1.66 & 0.94 & 0.00 & 0.42 & 34.72 & 2.66 & 30.65 & 0.17 & 0.05 & 0.14 & 0.02 & 0.11 & 0.56 & 1.48 & 0.25 & 0.16 & 0.15 & 0.21 & 0.13 \\
\hline \begin{tabular}{l|l}
15 & $\mu$ \\
15
\end{tabular} & 19.91 & 340.17 & 3.74 & 1.78 & 1.13 & 1.20 & 0.83 & 39.71 & 3.86 & 34.30 & 0.21 & 0.35 & 0.13 & 0.03 & 0.15 & 0.96 & 0.89 & 0.18 & 0.54 & 0.00 & 0.38 & 0.17 \\
\hline$\sigma$ & 25.55 & 480.07 & 4.13 & 0.24 & 0.89 & 1.32 & 0.17 & 54.18 & 5.70 & 48.93 & 0.37 & 0.09 & 0.22 & 0.02 & 0.23 & 1.04 & 1.71 & 0.26 & 0.09 & 0.02 & 0.60 & 0.27 \\
\hline$\underline{\mu}$ & 25.26 & 140.42 & 3.48 & 1.38 & 0.50 & 0.31 & 0.74 & 56.59 & 10.68 & 120.24 & 0.37 & 0.39 & 0.21 & 0.02 & 0.23 & 1.19 & 2.63 & 0.18 & 0.51 & 0.05 & 0.60 & 0.24 \\
\hline$\sigma$ & 6.84 & 73.81 & 0.93 & 0.30 & 0.23 & 0.05 & 0.16 & 24.82 & 1.97 & 24.32 & 0.07 & 0.22 & 0.07 & 0.03 & 0.04 & 0.11 & 2.24 & 0.33 & 0.17 & 0.12 & 0.07 & 0.03 \\
\hline
\end{tabular}

Table 15: Frequency domain parameters SESSION 4 - SESSION 1 difference for EO Binaural $f_{0}$ therapy 
$\underline{\text { Time domain parameters }}$

\begin{tabular}{|c|c|c|c|c|c|c|c|c|c|c|c|c|c|c|c|c|c|c|c|c|c|}
\hline Channel & Mean & MeanAbs & Median & Max & $\min$ & Peak & SSI & IEEG & $\mathrm{WL}$ & MMAV1 & \begin{tabular}{|l|} 
MMAV2 \\
\end{tabular} & STD DEV & VAR & ROOT & RMS & CREST & SKEWNESS & KURTOSIS & CLEARANCE & SHAPE & IMPULSE \\
\hline \multirow{2}{*}{1} & 9.69 & 0.46 & $-\quad 0.12$ & -0.04 & -0.53 & -0.16 & -0.58 & -0.46 & -0.53 & -0.47 & \begin{tabular}{|l|}
-0.47 \\
\end{tabular} & -0.45 & -0.58 & -0.47 & -0.45 & 0.65 & $\begin{array}{ll}-2.28 \\
\end{array}$ & 0.24 & \begin{tabular}{|l|}
0.70 \\
\end{tabular} & 0.02 & 0.68 \\
\hline & 13.68 & 0.49 & 1.09 & 0.79 & 0.38 & 0.68 & 0.55 & 0.49 & 0.45 & 0.49 & 0.47 & 0.50 & 0.55 & 0.49 & 0.50 & 0.27 & 0.12 & 0.02 & 0.29 & 0.00 & 0.28 \\
\hline \multirow{2}{*}{2} & 23.09 & 0.70 & 144.93 & 1.05 & 0.24 & 0.52 & 3.38 & 0.70 & -0.58 & 0.80 & 1.32 & 0.74 & 3.32 & 0.68 & 0.75 & -0.10 & 4.70 & 0.14 & 0.06 & 0.03 & 0.07 \\
\hline & 13.82 & 1.58 & 257.76 & 1.87 & 1.08 & 1.33 & 5.68 & 1.58 & 0.39 & 1.66 & 2.12 & 1.61 & 5.60 & 1.55 & 1.62 & 0.07 & 5.47 & 0.03 & 0.08 & 0.00 & 0.08 \\
\hline \multirow{2}{*}{3} & 3.35 & 0.35 & 2.48 & -0.11 & -0.34 & -0.23 & -0.35 & -0.35 & -0.42 & 0.36 & 0.37 & 0.33 & -0.35 & -0.35 & -0.33 & 0.28 & 1.69 & .25 & 0.31 & 0.02 & 0.30 \\
\hline & 7.83 & 0.63 & 3.87 & 0.82 & 0.54 & 0.66 & 0.86 & 0.63 & 0.57 & 0.61 & 0.60 & 0.64 & 0.86 & 0.62 & 0.64 & 0.24 & 0.04 & .02 & 0.24 & 0.00 & 0.24 \\
\hline \multirow{2}{*}{4} & 171.52 & 0.37 & 0.75 & -0.36 & -0.48 & -0.37 & -0.50 & -0.37 & -0.53 & 0.36 & 0.33 & 0.37 & -0.50 & -0.36 & -0.37 & 0.01 & 1.12 & 0.06 & 0.00 & 0.01 & 0.00 \\
\hline & 225.86 & 0.46 & 4.43 & 0.46 & 0.37 & 0.45 & 0.58 & 0.46 & 0.36 & 0.47 & 0.50 & 0.46 & 0.58 & 0.46 & 0.46 & 0.03 & 0.51 & 0.03 & 0.02 & 0.01 & 0.03 \\
\hline \multirow{2}{*}{5} & 1.04 & 0.27 & 1.37 & -0.22 & -0.33 & -0.22 & -0.40 & -0.27 & -0.41 & 0.28 & 0.30 & 0.26 & -0.40 & -0.27 & -0.26 & 0.06 & 1.13 & 0.04 & 0.06 & 0.00 & 0.06 \\
\hline & 0.34 & 0.33 & 0.61 & 0.37 & 0.32 & 0.37 & 0.50 & 0.33 & 0.21 & 0.33 & 0.33 & 0.34 & 0.50 & 0.33 & 0.34 & 0.01 & 1.29 & 0.05 & 0.03 & 0.01 & 0.02 \\
\hline \multirow{2}{*}{65} & 0.32 & 0.42 & 1.83 & -0.33 & -0.30 & -0.33 & -0.58 & -0.42 & 0.52 & 0.43 & 0.43 & 0.41 & -0.58 & -0.43 & -0.41 & 0.13 & 0.90 & 0.12 & 0.15 & 0.01 & 0.14 \\
\hline & 0.97 & 0.38 & 1.32 & 0.46 & 0.47 & 0.46 & 0.45 & 0.38 & 0.26 & 0.37 & 0.36 & 0.39 & 0.45 & 0.37 & 0.39 & 0.03 & 0.88 & 0.04 & 0.05 & 0.01 & 0.04 \\
\hline \multirow{2}{*}{7} & 13.59 & 0.06 & 18.64 & -0.18 & 0.21 & 0.16 & 0.40 & 0.06 & 0.54 & 0.11 & 0.33 & 0.04 & 0.38 & 0.07 & 0.05 & 0.04 & 306.52 & .00 & .00 & 0.02 & 0.02 \\
\hline & 20.49 & 0.75 & 6.14 & 0.72 & 0.92 & 0.99 & 1.61 & 0.75 & 0.05 & 0.78 & 0.86 & 0.76 & 1.59 & 0.74 & 0.77 & 0.18 & 436.49 & 0.22 & 0.23 & 0.03 & 0.21 \\
\hline \multirow[b]{2}{*}{8} & 18.24 & 0.39 & 92.05 & -0.02 & -0.45 & -0.07 & -0.59 & -0.39 & -0.61 & 0.43 & 0.56 & 0.37 & -0.60 & -0.41 & -0.36 & 0.44 & 20.70 & 0.82 & 0.57 & 0.06 & 0.53 \\
\hline & 32.09 & 0.03 & 114.99 & 0.07 & 0.00 & 0.00 & 0.04 & 0.03 & 0.04 & 0.02 & 0.00 & 0.03 & 0.04 & 0.03 & 0.03 & 0.06 & 4.85 & 0.03 & 0.07 & 0.00 & 0.06 \\
\hline \multirow[b]{2}{*}{ c } & 4.30 & 0.43 & 7.14 & -0.40 & -0.53 & -0.46 & -0.69 & -0.43 & 0.38 & 0.45 & 0.48 & 0.44 & -0.68 & -0.43 & -0.44 & -0.04 & 1.77 & 0.09 & 0.06 & 0.01 & 0.05 \\
\hline & 4.81 & 0.04 & 11.34 & 0.03 & 0.10 & 0.05 & 0.05 & 0.04 & 0.16 & 0.03 & 0.00 & 0.05 & 0.06 & 0.04 & 0.05 & 0.01 & 1.54 & 0.04 & 0.02 & 0.00 & 0.02 \\
\hline \multirow{2}{*}{10} & 0.58 & 0.39 & 0.78 & -0.44 & -0.37 & -0.41 & -0.60 & -0.39 & -0.34 & 0.39 & 0.37 & 0.39 & -0.60 & -0.40 & -0.39 & -0.06 & 0.03 & 0.01 & 0.05 & 0.00 & 0.06 \\
\hline & 0.08 & 0.21 & 0.11 & 0.11 & 0.35 & 0.29 & 0.26 & 0.21 & 0.06 & 0.21 & 0.20 & 0.21 & 0.26 & 0.20 & 0.21 & 0.15 & 1.00 & 0.07 & 0.17 & 0.01 & 0.16 \\
\hline \multirow{2}{*}{11} & 2.89 & 0.25 & 13.83 & -0.34 & -0.30 & -0.34 & -0.34 & -0.25 & -0.41 & 0.25 & 0.26 & 0.26 & -0.35 & -0.24 & -0.26 & -0.09 & 6.94 & 0.05 & 0.11 & 0.01 & 0.10 \\
\hline & 5.03 & 0.45 & 20.26 & 0.36 & 0.38 & 0.36 & 0.67 & 0.45 & 0.08 & 0.46 & 0.46 & 0.45 & 0.66 & 0.45 & 0.45 & 0.06 & 8.64 & 0.01 & 0.06 & 0.00 & 0.06 \\
\hline \multirow{2}{*}{12} & 5.58 & 0.10 & 4.90 & -0.01 & -0.06 & -0.06 & 0.26 & 0.10 & 0.17 & 0.15 & 0.21 & 0.06 & 0.21 & 0.11 & 0.08 & -0.11 & 1.22 & 0.10 & 0.13 & 0.02 & 0.13 \\
\hline & 11.48 & 0.43 & 10.11 & 0.39 & 0.27 & 0.27 & 0.90 & 0.43 & 0.47 & 0.45 & 0.51 & 0.41 & 0.87 & 0.45 & 0.42 & 0.09 & 2.07 & 0.04 & 0.10 & 0.01 & 0.10 \\
\hline \multirow{2}{*}{13} & 1.60 & 0.14 & 1.50 & -0.34 & -0.34 & -0.34 & -0.27 & -0.14 & -0.13 & 0.13 & 0.19 & 0.15 & -0.27 & -0.13 & -0.15 & -0.21 & 1.00 & 0.21 & 0.23 & 0.02 & 0.22 \\
\hline & 1.16 & 0.11 & 0.82 & 0.04 & 0.05 & 0.05 & 0.16 & $\begin{array}{l}0.11 \\
\end{array}$ & 0.15 & 0.10 & $\begin{array}{ll}0.14 \\
\end{array}$ & 0.09 & 0.16 & 0.11 & 0.10 & 0.15 & 1.14 & 0.12 & 0.16 & 0.01 & 0.15 \\
\hline \multirow{2}{*}{14} & 24.74 & 0.45 & 1.59 & 0.26 & 0.38 & 0.38 & 1.01 & 0.45 & 0.00 & 0.52 & 0.70 & 0.37 & 0.88 & 0.47 & 0.42 & -0.02 & 0.74 & 0.11 & 0.06 & 0.02 & 0.04 \\
\hline & 21.46 & 0.10 & 14.16 & 0.07 & 0.02 & 0.02 & 0.25 & 0.10 & 0.26 & 0.11 & 0.07 & 0.08 & 0.23 & 0.10 & 0.09 & 0.05 & 0.40 & 0.05 & 0.05 & 0.01 & 0.05 \\
\hline \multirow{2}{*}{15} & 9.67 & 0.42 & 1.18 & 0.56 & 0.77 & 0.73 & 1.10 & 0.42 & 0.83 & 0.42 & 0.46 & 0.43 & 1.10 & 0.41 & 0.43 & 0.20 & 1.04 & 0.06 & 0.22 & 0.01 & 0.21 \\
\hline & 14.16 & 0.30 & 0.32 & 0.21 & 0.46 & 0.41 & 0.85 & 0.30 & 0.44 & 0.29 & 0.23 & 0.30 & 0.85 & 0.31 & 0.30 & 0.03 & 4.44 & 0.02 & 0.02 & 0.01 & 0.03 \\
\hline \multirow{2}{*}{$16 \frac{\mu}{\sigma}$} & 13.23 & 0.84 & 18.68 & 0.61 & 0.06 & 0.13 & 2.10 & 0.84 & 0.11 & 0.89 & 0.94 & 0.72 & 1.98 & 0.90 & 0.75 & -0.36 & 4.48 & 0.21 & 0.41 & 0.05 & 0.39 \\
\hline & 6.74 & 0.29 & 55.19 & 0.24 & 0.21 & 0.23 & 0.91 & 0.29 & 0.19 & 0.31 & 0.34 & 0.26 & 0.88 & 0.32 & 0.26 & 0.04 & 1.90 & 0.01 & 0.02 & 0.01 & 0.03 \\
\hline
\end{tabular}

Table 16: Time domain parameters SESSION 4 - SESSION 1 difference for EO Binaural $f_{0}$ therapy 
- TRT therapy (data from 6 patients)

Frequency domain parameters

\begin{tabular}{|c|c|c|c|c|c|c|c|c|c|c|c|c|c|c|c|c|c|c|c|c|c|c|}
\hline \multirow{2}{*}{$\begin{array}{l}\text { Channe } \\
\mu\end{array}$} & PSDtotal & PSD- $\delta$ & PSD- $\theta$ & PSD- $\alpha$ & PSD- $\beta$ & PSD- $\varnothing$ & $P 1(X m f)$ & P2 & P3 & P4 & $\mathrm{P} 5(\mathrm{Xfc})$ & P6 & P7(Xrmsf) & P8 & P9 & P10 & P11 & P12 & P13 & P14 & MDF & PSDef \\
\hline & 1.02 & 2.43 & 0.39 & 0.30 & 0.36 & 0.48 & -0.15 & 0.37 & 0.89 & 5.98 & 0.07 & -0.07 & 0.02 & 0.00 & 0.03 & 0.04 & 0.41 & 0.23 & -0.12 & 0.04 & 0.63 & 0.00 \\
\hline$\sqrt[1]{\sigma}$ & 0.66 & 2.57 & 0.26 & 0.25 & 0.22 & 0.29 & 0.35 & 1.40 & 1.50 & 7.75 & 0.42 & 0.18 & 0.19 & 0.02 & 0.22 & 0.33 & 0.83 & 0.44 & 0.27 & 0.04 & 1.96 & 0.14 \\
\hline$\mu$ & 0.59 & 1.12 & 0.45 & 0.32 & 0.37 & 0.59 & 0.15 & 0.19 & 0.15 & 0.08 & 0.26 & 0.09 & 0.09 & -0.02 & 0.13 & 0.14 & 0.13 & 0.12 & -0.12 & 0.02 & 1.41 & 0.06 \\
\hline $2 \sigma$ & 0.21 & 1.09 & 0.27 & 0.27 & 0.11 & 0.34 & 0.31 & 0.67 & 0.48 & 1.19 & 0.71 & 0.15 & 0.30 & 0.03 & 0.35 & 0.31 & 0.60 & 0.40 & 0.23 & 0.08 & 3.54 & 0.16 \\
\hline$\mu$ & 7.14 & 26.56 & 2.71 & 1.03 & 0.55 & 0.38 & 0.05 & 12.91 & 1.10 & 4.47 & -0.06 & 0.00 & 0.05 & -0.00 & -0.05 & 0.29 & 0.82 & 0.17 & 0.01 & 0.01 & 0.47 & 0.06 \\
\hline$\sqrt[3]{\sigma}$ & 14.34 & 56.81 & 5.86 & 1.95 & 0.71 & 0.19 & 0.57 & 29.64 & 1.65 & 7.52 & 0.37 & 0.22 & 0.19 & 0.03 & 0.22 & 0.86 & 1.29 & 0.30 & 0.37 & 0.03 & 1.97 & 0.20 \\
\hline$\mu$ & 0.65 & 0.83 & 0.45 & 0.34 & 0.33 & 0.46 & 0.22 & 0.15 & 0.63 & 4.56 & -0.01 & 0.13 & 0.02 & -0.01 & 0.01 & 0.09 & 0.63 & 0.39 & -0.17 & -0.00 & 0.12 & 0.00 \\
\hline$\sqrt[4]{\sigma}$ & 0.23 & 0.34 & 0.22 & 0.16 & 0.12 & 0.23 & 0.19 & 0.73 & 1.38 & 8.62 & 0.23 & 0.10 & 0.12 & 0.02 & 0.14 & 0.21 & 0.69 & 0.42 & 0.14 & 0.04 & 0.80 & 0.09 \\
\hline$\mu$ & 0.56 & 0.64 & 0.48 & 0.36 & 0.29 & 0.33 & 0.19 & 0.31 & -0.19 & 0.15 & 0.15 & -0.12 & 0.05 & -0.01 & 0.07 & -0.17 & 0.13 & 0.22 & -0.15 & -0.01 & 0.72 & 0.05 \\
\hline$\sqrt[5]{\sigma}$ & 0.29 & 0.30 & 0.24 & 0.16 & 0.16 & 0.18 & 0.25 & 0.60 & 0.55 & 1.40 & 0.34 & 0.14 & 0.16 & 0.01 & 0.17 & 0.27 & 0.38 & 0.38 & 0.20 & 0.05 & 1.65 & 0.10 \\
\hline$\mu$ & 0.56 & 1.71 & 0.36 & 0.22 & 0.25 & 0.32 & 0.13 & 0.15 & 0.06 & 0.08 & 0.01 & 0.09 & 0.01 & -0.01 & 0.01 & 0.07 & 0.20 & 0.21 & -0.10 & -0.02 & 0.11 & -0.01 \\
\hline$\sqrt[6]{\sigma}$ & 0.18 & 2.04 & 0.19 & 0.10 & 0.09 & 0.17 & 0.16 & 0.69 & 0.54 & 1.14 & 0.16 & 0.09 & 0.09 & 0.02 & 0.10 & 0.19 & 0.33 & 0.29 & 0.12 & 0.04 & 0.47 & 0.07 \\
\hline$\mu$ & 1.22 & 6.12 & 0.68 & 0.39 & 0.27 & 0.30 & 0.04 & 1.02 & 1.92 & 20.13 & -0.03 & -0.03 & 0.03 & -0.02 & 0.01 & 0.06 & 0.27 & 0.12 & -0.04 & -0.01 & 0.12 & 0.02 \\
\hline$\sigma$ & 0.76 & 6.17 & 0.32 & 0.22 & 0.16 & 0.17 & 0.21 & 1.69 & 3.41 & 38.79 & 0.25 & 0.12 & 0.12 & 0.03 & 0.15 & 0.31 & 0.51 & 0.19 & 0.16 & 0.03 & 0.76 & 0.11 \\
\hline$\mu$ & 2.02 & 9.35 & 0.49 & 0.52 & 0.50 & 0.54 & 0.07 & 2.54 & 1.88 & 18.61 & 0.04 & 0.06 & 0.00 & -0.02 & 0.03 & -0.04 & 0.66 & 0.15 & -0.06 & -0.01 & 0.36 & -0.02 \\
\hline$\sqrt[8]{\sigma}$ & 3.02 & 20.93 & 0.21 & 0.34 & 0.28 & 0.48 & 0.37 & 6.82 & 5.10 & 45.98 & 0.23 & 0.22 & 0.11 & 0.03 & 0.14 & 0.37 & 1.65 & 0.37 & 0.28 & 0.06 & 1.01 & 0.10 \\
\hline$\mu$ & 0.59 & 0.97 & 0.50 & 0.27 & 0.30 & 0.42 & 0.10 & 0.11 & 0.02 & 0.28 & -0.05 & -0.07 & 0.04 & -0.01 & 0.03 & 0.02 & 0.61 & 0.30 & -0.08 & -0.02 & 0.11 & 0.01 \\
\hline 9 & 0.19 & 0.60 & 0.16 & 0.11 & 0.20 & 0.20 & 0.25 & 0.83 & 0.61 & 1.19 & 0.08 & 0.16 & 0.06 & 0.01 & 0.05 & 0.19 & 0.93 & 0.47 & 0.20 & 0.04 & 0.12 & 0.03 \\
\hline$\mu$ & 0.76 & 2.73 & 0.58 & 0.46 & 0.31 & 0.36 & 0.07 & 0.12 & 0.27 & 2.40 & -0.01 & -0.06 & 0.02 & -0.01 & 0.01 & 0.02 & 0.20 & 0.16 & -0.06 & -0.02 & 0.01 & 0.00 \\
\hline$1 0 \longdiv { \sigma }$ & 0.37 & 4.52 & 0.32 & 0.37 & 0.24 & 0.13 & 0.19 & 1.02 & 1.03 & 4.68 & 0.16 & 0.10 & 0.10 & 0.02 & 0.09 & 0.22 & 0.36 & 0.26 & 0.14 & -0.01 & 0.28 & 0.06 \\
\hline \multirow{2}{*}{11} & 0.57 & 0.85 & 0.29 & 0.24 & 0.28 & 0.33 & 0.17 & 0.36 & 0.02 & 1.69 & 0.01 & -0.10 & 0.00 & -0.00 & 0.00 & -0.10 & 0.15 & 0.24 & -0.13 & -0.02 & 0.01 & -0.01 \\
\hline & 0.25 & 0.37 & 0.11 & 0.07 & 0.08 & 0.15 & 0.10 & 0.48 & 1.31 & 5.96 & 0.11 & 0.07 & 0.06 & 0.01 & 0.06 & 0.15 & 0.27 & 0.14 & 0.08 & 0.04 & 0.20 & 0.05 \\
\hline$\mu$ & 0.94 & 1.50 & 0.98 & 0.68 & 0.45 & 0.46 & 0.16 & 0.35 & 0.28 & 0.81 & 0.11 & -0.08 & 0.04 & -0.01 & 0.06 & 0.09 & 0.75 & 0.11 & -0.13 & 0.02 & 0.82 & -0.03 \\
\hline$\sigma$ & 0.77 & 1.79 & 1.09 & 0.75 & 0.15 & 0.19 & 0.28 & 2.04 & 1.03 & 2.05 & 0.33 & 0.15 & 0.17 & 0.02 & 0.19 & 0.35 & 2.00 & 0.28 & 0.22 & 0.03 & 1.88 & 0.12 \\
\hline \multirow{2}{*}{ 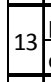 } & 0.46 & 0.60 & 0.34 & 0.26 & 0.29 & 0.28 & 0.10 & 0.21 & -0.11 & 0.01 & 0.06 & 0.06 & 0.02 & -0.01 & 0.04 & -0.09 & 0.08 & 0.07 & -0.08 & -0.00 & 0.24 & -0.03 \\
\hline & 0.20 & 0.24 & 0.14 & 0.09 & 0.13 & 0.11 & 0.15 & 0.52 & 0.50 & 1.12 & 0.19 & 0.08 & 0.10 & 0.02 & 0.12 & 0.18 & 0.36 & 0.20 & 0.11 & 0.04 & 0.61 & 0.07 \\
\hline$\mu$ & 0.74 & 1.41 & 0.51 & 0.39 & 0.29 & 0.41 & 0.10 & 0.35 & 0.66 & 3.53 & -0.01 & -0.06 & 0.02 & -0.01 & -0.01 & -0.01 & 0.48 & 0.18 & -0.08 & -0.01 & 0.00 & 0.01 \\
\hline$\sigma$ & 0.58 & 1.57 & 0.35 & 0.29 & 0.13 & 0.17 & 0.10 & 1.59 & 1.76 & 7.57 & 0.23 & 0.05 & 0.13 & 0.01 & 0.13 & 0.24 & 0.84 & 0.20 & 0.07 & 0.03 & 0.46 & 0.09 \\
\hline$\mu$ & 0.59 & 0.61 & 0.48 & 0.35 & 0.33 & 0.45 & 0.18 & 0.48 & 0.28 & 0.34 & 0.11 & -0.10 & 0.05 & -0.01 & 0.06 & -0.17 & 1.18 & 0.17 & -0.14 & -0.00 & 0.36 & -0.04 \\
\hline$\sigma$ & 0.25 & 0.28 & 0.18 & 0.16 & 0.11 & 0.28 & 0.24 & 0.45 & 0.33 & 0.59 & 0.20 & 0.14 & 0.11 & 0.02 & 0.11 & 0.18 & 2.45 & 0.28 & 0.18 & 0.02 & 0.59 & 0.07 \\
\hline \multirow[b]{2}{*}{ jo } & 0.57 & 0.99 & 0.34 & 0.31 & 0.28 & 0.33 & 0.17 & 0.29 & $\begin{array}{ll}0.14 \\
\end{array}$ & 1.43 & 0.01 & -0.09 & 0.00 & -0.01 & 0.00 & 0.09 & 0.78 & 0.17 & 0.13 & -0.01 & 0.05 & -0.01 \\
\hline & 0.18 & 1.00 & 0.15 & 0.06 & 0.10 & 0.17 & 0.14 & 0.66 & 1.29 & 4.90 & 0.13 & 0.10 & 0.08 & 0.02 & 0.07 & 0.15 & 1.69 & 0.29 & 0.11 & 0.03 & 0.28 & 0.05 \\
\hline
\end{tabular}

Table 17: Frequency domain parameters SESSION 4 - SESSION 1 difference for EO TRT therapy 
$\underline{\text { Time domain parameters }}$

\begin{tabular}{|c|c|c|c|c|c|c|c|c|c|c|c|c|c|c|c|c|c|c|c|c|c|}
\hline Chann & Mean & MeanAbs & Median & $\operatorname{Max}$ & $\min$ & Peak & SSI & IEEG & WL & MMAV1 & \begin{tabular}{|l|} 
MMAV2 \\
\end{tabular} & STD DEV & VAR & ROOT & RMS & CREST & SKEWNESS & KURTOSIS & CLEARANCE & SHAPE & IMPULSE \\
\hline \multirow{2}{*}{\begin{tabular}{l|l|l} 
& $\mu$ \\
\end{tabular}} & 8.66 & 0.11 & 2.09 & -0.22 & -0.27 & -0.23 & 0.01 & -0.11 & -0.17 & -0.13 & -0.10 & -0.19 & -0.17 & -0.10 & -0.13 & 0.01 & 10.39 & 0.02 & 0.02 & -0.02 & 0.00 \\
\hline & 27.32 & 0.57 & 7.94 & 0.42 & 0.33 & 0.39 & 1.00 & 0.57 & 0.34 & 0.55 & 0.61 & 0.45 & 0.64 & 0.59 & 0.54 & 0.30 & 22.37 & 0.19 & 0.28 & 0.05 & 0.29 \\
\hline \multirow{2}{*}{2} & 8.32 & 0.19 & 3.79 & -0.24 & -0.19 & -0.25 & -0.13 & -0.19 & -0.06 & 0.19 & 0.17 & 0.19 & -0.13 & -0.19 & -0.19 & -0.13 & 11.16 & 0.15 & 0.14 & 0.02 & 0.14 \\
\hline & 20.88 & 0.50 & 8.32 & 0.63 & 0.63 & 0.63 & 0.89 & 0.50 & 0.35 & 0.51 & 0.54 & 0.51 & 0.89 & 0.50 & 0.51 & 0.25 & 19.94 & 0.27 & 0.26 & 0.03 & 0.26 \\
\hline \multirow[b]{2}{*}{3} & 0.53 & 42 & 0.96 & 0.24 & 0.16 & 0.26 & 2.45 & 0.42 & -0.23 & 0.42 & 0.52 & 41 & 2.29 & 0.41 & 0.44 & -0.04 & 0.36 & 0.06 & 0.04 & 0.00 & 0.04 \\
\hline & 8.95 & 1.24 & 6.35 & 1.06 & 0.81 & 1.09 & 5.22 & 1.24 & 0.16 & 1.21 & 1.18 & 1.24 & 4.86 & 1.21 & 1.29 & 0.25 & 2.10 & .15 & 0.26 & 0.04 & 0.25 \\
\hline \multirow{2}{*}{4} & 1.05 & 0.09 & 2.15 & -0.11 & -0.14 & -0.13 & 0.09 & -0.09 & -0.30 & 0.09 & 0.03 & 0.09 & 0.08 & -0.09 & -0.08 & 0.00 & 1.92 & 0.03 & 0.01 & 0.00 & 0.00 \\
\hline & 2.75 & 0.54 & 2.89 & 0.53 & 0.49 & 0.51 & 0.95 & 0.54 & 0.19 & 0.54 & 0.49 & 0.56 & 0.95 & 0.54 & 0.55 & 0.18 & 1.92 & 0.15 & 0.17 & 0.03 & 0.17 \\
\hline \multirow{2}{*}{5} & 2.74 & 0.28 & 0.42 & -0.29 & -0.18 & -0.23 & -0.21 & -0.28 & -0.20 & 0.28 & 0.26 & 0.28 & -0.22 & -0.29 & -0.27 & 0.14 & 3.66 & 0.10 & 0.17 & 0.02 & 0.16 \\
\hline & 6.81 & 0.54 & 2.09 & 0.42 & 0.58 & 0.54 & 0.89 & 0.54 & 0.15 & 0.54 & 0.52 & 0.55 & 0.88 & 0.53 & 0.55 & 0.17 & 9.42 & 0.10 & 0.20 & 0.03 & 0.18 \\
\hline & 1.80 & 0.22 & 2.24 & -0.08 & -0.23 & -0.19 & -0.28 & -0.22 & 0.20 & 0.23 & 0.22 & 0.23 & -0.28 & -0.22 & -0.22 & 0.07 & 0.67 & 0.09 & 0.06 & 0.00 & 0.07 \\
\hline & 0.93 & 0.37 & 1.65 & 0.54 & 0.34 & 0.39 & 0.54 & 0.37 & 0.14 & 0.36 & 0.33 & 0.39 & 0.54 & 0.37 & 0.38 & 0.17 & 4.27 & 0.27 & 0.16 & 0.01 & $\begin{array}{ll}0.17 \\
\end{array}$ \\
\hline \multirow{2}{*}{7} & 0.78 & 0.52 & 12.75 & 0.61 & 0.08 & 0.22 & 2.60 & 0.52 & 0.17 & 0.58 & 1.49 & 0.52 & 2.60 & 0.53 & 0.51 & -0.0 & 3.06 & 1.02 & .12 & 0.02 & 0.10 \\
\hline & 3.02 & 1.25 & 18.84 & 1.02 & 0.62 & 0.73 & 5.49 & 1.25 & 0.15 & 1.33 & 2.26 & 1.24 & 5.49 & 1.23 & 1.25 & 0.27 & 4.20 & 0.32 & 0.24 & 0.09 & 0.24 \\
\hline \multirow{2}{*}{8} & 3.53 & 0.27 & 10.28 & 0.09 & 0.03 & 0.03 & 1.73 & 0.27 & -0.03 & 0.25 & 0.17 & 0.20 & 1.70 & 0.32 & 0.20 & 0.01 & 2.37 & 0.04 & 0.02 & 0.02 & 0.01 \\
\hline & 4.76 & 1.38 & 20.80 & 1.07 & 0.64 & 0.76 & 4.97 & 1.38 & 0.34 & 1.36 & 1.30 & 1.23 & 4.89 & 1.49 & 1.24 & 0.22 & 5.51 & 0.16 & 0.26 & 0.04 & 0.24 \\
\hline & 0.30 & 0.08 & 2.42 & -0.26 & 0.00 & -0.11 & 0.21 & 0.08 & 0.25 & 0.08 & 0.14 & 0.13 & -0.18 & 0.11 & 0.04 & -0.09 & 17.03 & 0.02 & 0.11 & 0.02 & 0.10 \\
\hline & 3.02 & 0.49 & 5.71 & 0.37 & 0.36 & 0.21 & 0.91 & 0.49 & 0.18 & 0.50 & 0.57 & 0.27 & 0.48 & 0.56 & 0.40 & 0.19 & 25.57 & 0.12 & 0.24 & 0.06 & 0.22 \\
\hline \multirow{2}{*}{10} & 4.65 & 0.21 & 2.52 & -0.06 & 0.38 & 0.14 & 0.80 & 0.21 & -0.15 & 0.23 & 0.23 & 0.17 & 0.74 & 0.20 & 0.21 & -0.02 & 1.82 & 0.03 & 0.01 & 0.00 & 0.01 \\
\hline & 9.60 & 0.63 & 3.98 & 0.52 & 0.71 & 0.52 & 1.72 & 0.63 & 0.18 & 0.66 & 0.73 & 0.67 & 1.75 & 0.63 & 0.64 & 0.24 & 7.68 & 0.22 & 0.27 & 0.02 & 0.26 \\
\hline \multirow{2}{*}{11} & 0.51 & 0.25 & 0.40 & -0.22 & -0.22 & -0.23 & -0.30 & -0.25 & -0.20 & 0.26 & 0.25 & 0.25 & -0.30 & -0.26 & -0.25 & 0.08 & 0.13 & 0.00 & 0.07 & 0.01 & 0.07 \\
\hline & 1.13 & 0.40 & 0.72 & 0.37 & 0.38 & 0.37 & 0.65 & 0.40 & 0.10 & 0.39 & 0.38 & 0.41 & 0.65 & 0.39 & 0.42 & 0.17 & 1.72 & 0.15 & 0.16 & 0.02 & 0.17 \\
\hline \multirow{2}{*}{12} & 1.17 & 0.17 & 0.43 & -0.16 & -0.15 & -0.14 & 0.00 & -0.17 & -0.17 & 0.17 & 0.21 & 0.16 & 0.01 & -0.18 & -0.15 & 0.08 & 4.61 & 0.09 & 0.10 & 0.01 & 0.09 \\
\hline & 0.89 & 0.55 & 3.96 & 0.72 & 0.48 & 0.61 & 1.14 & 0.55 & 0.27 & 0.53 & 0.45 & 0.60 & 1.15 & 0.54 & 0.59 & 0.21 & 6.84 & 0.33 & 0.23 & 0.05 & 0.22 \\
\hline \multirow{2}{*}{13} & 0.62 & 0.09 & 3.29 & -0.18 & -0.06 & -0.13 & -0.06 & -0.09 & -0.12 & 0.13 & 0.22 & 0.10 & 0.07 & -0.09 & -0.09 & -0.06 & 1.81 & 0.09 & 0.07 & 0.01 & 0.07 \\
\hline & 3.33 & 0.35 & 8.85 & 0.33 & 0.41 & 0.38 & 0.59 & 0.35 & 0.10 & 0.33 & 0.28 & 0.39 & 0.60 & 0.35 & 0.37 & 0.08 & 1.88 & 0.22 & 0.11 & 0.03 & 0.10 \\
\hline \multirow{2}{*}{14} & 7.93 & 0.03 & 2.11 & 0.02 & -0.00 & 0.01 & 0.26 & 0.03 & -0.16 & 0.02 & 0.01 & 0.03 & 0.23 & 0.02 & 0.05 & 0.01 & 29.93 & 0.24 & 0.04 & 0.02 & 0.03 \\
\hline & 21.11 & 0.43 & 8.61 & 0.32 & 0.50 & 0.41 & 0.82 & 0.43 & 0.16 & 0.41 & 0.35 & 0.44 & 0.81 & 0.43 & 0.44 & 0.24 & 68.49 & 0.44 & 0.25 & 0.02 & 0.25 \\
\hline \multirow{2}{*}{15} & 1.61 & 0.38 & 1.46 & -0.29 & -0.35 & -0.33 & -0.52 & -0.38 & -0.14 & 0.38 & 0.34 & 0. & -0.53 & -0.38 & -0.38 & 0.09 & 0.69 & 0.05 & .09 & 0.00 & 0.08 \\
\hline & 8.27 & 0.33 & 0.93 & 0.41 & 0.36 & 0.39 & 0.37 & 0.33 & 0.30 & 0.34 & 0.28 & 0. & 0.37 & 0.33 & 0.34 & 0.11 & 0.74 & 0.19 & 0.12 & 0.02 & 0.11 \\
\hline \multirow{2}{*}{$16 \frac{\mu}{\sigma}$} & 6.21 & 0.02 & 1.28 & 0.06 & -0.07 & 0.02 & 0.07 & -0.02 & -0.19 & 0.00 & 0.11 & 0.03 & 0.05 & -0.02 & -0.02 & 0.04 & 1.02 & 0.01 & 0.04 & 0.00 & 0.04 \\
\hline & 15.89 & 0.33 & 3.49 & 0.45 & 0.32 & 0.37 & 0.76 & 0.33 & 0.14 & 0.31 & 0.37 & 0.36 & 0.76 & 0.31 & 0.35 & 0.16 & 2.65 & 0.16 & 0.21 & 0.04 & 0.19 \\
\hline
\end{tabular}

Table 18: Time domain parameters SESSION 4 - SESSION 1 difference for EO TRT therapy 
- EAE therapy (data from 4 patients)

Frequency domain parameters

\begin{tabular}{|c|c|c|c|c|c|c|c|c|c|c|c|c|c|c|c|c|c|c|c|c|c|c|}
\hline \multirow{2}{*}{ Channe } & PSDtotal & PSD- $\delta$ & PSD- $\theta$ & PSD- $\alpha$ & PSD- $\beta$ & PSD- $\varnothing$ & $P 1(X m f)$ & P2 & P3 & P4 & $\mathrm{P} 5(\mathrm{Xfc})$ & P6 & $\mathrm{P7}$ (Xrmsf) & P8 & P9 & P10 & P11 & P12 & P13 & P14 & MDF & PSDef \\
\hline & 1.23 & 3.63 & 0.41 & 0.28 & 0.31 & 0.38 & -0.24 & 0.83 & 2.26 & 20.01 & 0.55 & -0.10 & 0.17 & -0.03 & 0.22 & 0.09 & 0.31 & 0.08 & -0.19 & 0.12 & 9.03 & -0.07 \\
\hline$\sqrt[1]{\sigma}$ & 0.69 & 3.54 & 0.32 & 0.22 & 0.14 & 0.16 & 0.39 & 1.78 & 2.94 & 28.12 & 1.38 & 0.20 & 0.51 & 0.03 & 0.57 & 0.52 & 0.66 & 0.25 & 0.32 & 0.20 & 18.46 & 0.27 \\
\hline$\mu$ & 1.19 & 3.24 & 0.60 & 0.40 & 0.39 & 0.46 & 0.34 & 0.44 & 0.42 & 2.67 & 0.43 & 0.19 & 0.15 & -0.03 & 0.19 & 0.20 & 0.22 & 0.34 & -0.28 & 0.06 & 6.42 & 0.08 \\
\hline $2 \sigma$ & 0.94 & 4.93 & 0.28 & 0.25 & 0.15 & 0.08 & 0.38 & 2.38 & 1.46 & 6.06 & 0.96 & 0.22 & 0.37 & 0.05 & 0.46 & 0.51 & 0.61 & 0.19 & 0.32 & 0.12 & 13.05 & 0.24 \\
\hline$\mu$ & 1.41 & 4.49 & 0.49 & 0.25 & 0.64 & 0.82 & 0.24 & 1.47 & 1.56 & 16.53 & 0.06 & 0.09 & 0.03 & -0.01 & 0.03 & 0.07 & 0.22 & 0.13 & 0.16 & -0.01 & 0.22 & -0.02 \\
\hline$\sqrt[3]{\sigma}$ & 1.48 & 7.82 & 0.22 & 0.06 & 0.50 & 0.69 & 0.36 & 2.76 & 2.99 & 32.21 & 0.22 & 0.19 & 0.12 & 0.02 & 0.11 & 0.31 & 0.57 & 0.23 & 0.26 & 0.04 & 0.52 & 0.07 \\
\hline$\mu$ & 1.45 & 4.80 & 0.44 & 0.42 & 0.27 & 0.31 & 0.05 & 1.63 & 1.09 & 10.38 & 0.21 & 0.03 & 0.08 & -0.02 & 0.11 & 0.04 & 0.08 & 0.05 & 0.04 & 0.01 & 1.46 & -0.05 \\
\hline$\sqrt[4]{\sigma}$ & 1.93 & 8.42 & 0.26 & 0.22 & 0.07 & 0.19 & 0.35 & 3.97 & 2.92 & 21.65 & 0.57 & 0.20 & 0.25 & 0.03 & 0.30 & 0.48 & 0.46 & 0.08 & 0.27 & 0.04 & 3.16 & 0.17 \\
\hline$\mu$ & 0.56 & 0.63 & 0.44 & 0.31 & 0.25 & 0.20 & 0.16 & 0.43 & -0.32 & 0.24 & 0.25 & -0.10 & 0.11 & -0.01 & 0.12 & -0.21 & 0.19 & 0.05 & -0.13 & -0.01 & 0.89 & 0.08 \\
\hline$\sqrt[5]{\sigma}$ & 0.31 & 0.30 & 0.32 & 0.25 & 0.12 & 0.03 & 0.24 & 0.48 & 0.46 & 0.82 & 0.41 & 0.13 & 0.19 & 0.01 & 0.20 & 0.28 & 0.30 & 0.05 & 0.18 & 0.01 & 1.58 & 0.11 \\
\hline$\mu$ & 0.83 & 1.67 & 0.44 & 0.36 & 0.26 & 0.26 & 0.23 & 0.07 & 0.08 & 1.03 & 0.35 & 0.14 & 0.13 & -0.03 & 0.18 & 0.22 & 0.11 & 0.16 & -0.19 & 0.02 & 3.80 & -0.09 \\
\hline$\sqrt[6]{\sigma}$ & 0.47 & 1.28 & 0.34 & 0.26 & 0.15 & 0.09 & 0.39 & 0.96 & 0.92 & 2.22 & 0.69 & 0.24 & 0.26 & 0.05 & 0.35 & 0.39 & 0.24 & 0.23 & 0.32 & 0.05 & 7.60 & 0.18 \\
\hline$\mu$ & 0.70 & 0.83 & 0.42 & 0.32 & 0.20 & 0.18 & 0.17 & 0.24 & 0.17 & 1.83 & 0.27 & -0.10 & 0.11 & -0.02 & 0.14 & 0.21 & 0.20 & 0.05 & -0.14 & -0.00 & 2.20 & 0.09 \\
\hline$\sigma$ & 0.26 & 0.12 & 0.29 & 0.19 & 0.06 & 0.02 & 0.27 & 0.86 & 1.18 & 4.90 & 0.45 & 0.15 & 0.19 & 0.03 & 0.23 & 0.32 & 0.40 & 0.06 & 0.21 & $\begin{array}{l}0.01 \\
\end{array}$ & 4.14 & 0.13 \\
\hline$\mu$ & 0.88 & 0.62 & 0.40 & 0.59 & 0.78 & 1.03 & 0.09 & 0.06 & -0.07 & 0.82 & 0.23 & 0.01 & 0.11 & -0.00 & 0.12 & -0.13 & 0.51 & -0.11 & 0.05 & -0.01 & 1.06 & -0.08 \\
\hline$\sqrt[8]{\sigma}$ & 0.68 & 0.24 & 0.14 & 0.44 & 0.98 & 0.93 & 0.45 & 0.93 & 0.60 & 2.56 & 0.32 & 0.19 & 0.14 & 0.03 & 0.17 & 0.28 & 0.31 & 0.22 & 0.32 & 0.03 & 1.82 & 0.10 \\
\hline$\mu$ & 0.49 & 0.88 & 0.47 & 0.36 & 0.33 & 0.30 & 0.12 & 0.21 & -0.12 & 0.02 & 0.30 & -0.08 & 0.13 & -0.01 & 0.15 & 0.18 & 0.21 & 0.01 & -0.10 & 0.00 & 1.91 & 0.08 \\
\hline 9 & 0.35 & 0.46 & 0.29 & 0.24 & 0.12 & 0.18 & 0.30 & 0.64 & 0.49 & 0.74 & 0.57 & 0.16 & 0.25 & 0.02 & 0.28 & 0.34 & 0.43 & 0.12 & 0.23 & 0.02 & 3.75 & 0.15 \\
\hline \multirow[b]{2}{*}{10} & 0.50 & 0.67 & 0.28 & 0.19 & 0.25 & 0.37 & 0.05 & 0.43 & -0.38 & 0.51 & 0.12 & 0.04 & 0.06 & -0.01 & 0.07 & -0.14 & 0.14 & 0.01 & -0.04 & 0.02 & 0.31 & -0.05 \\
\hline & 0.19 & 0.14 & 0.11 & 0.09 & 0.08 & 0.34 & 0.17 & 0.30 & 0.29 & 0.46 & 0.11 & 0.08 & 0.06 & 0.01 & 0.06 & 0.11 & 0.21 & 0.18 & 0.12 & 0.01 & 0.32 & 0.04 \\
\hline$\mu$ & 0.75 & 2.67 & 0.36 & 0.35 & 0.41 & 0.37 & 0.17 & 0.40 & 1.66 & 20.91 & 0.32 & -0.11 & 0.11 & -0.03 & 0.16 & -0.13 & 0.39 & 0.17 & -0.15 & 0.03 & 3.94 & -0.07 \\
\hline$\sigma$ & 0.55 & 4.00 & 0.39 & 0.34 & 0.22 & 0.10 & 0.45 & 1.52 & 3.47 & 41.81 & 0.76 & 0.27 & 0.31 & 0.05 & 0.40 & 0.45 & 1.03 & 0.28 & 0.37 & 0.07 & 8.07 & 0.19 \\
\hline$\mu$ & 1.02 & 0.68 & 0.37 & 0.39 & 0.80 & 1.02 & 0.04 & 0.10 & 0.13 & 0.78 & 0.21 & -0.06 & 0.09 & -0.02 & 0.12 & 0.17 & 0.14 & 0.04 & -0.06 & -0.00 & 1.15 & -0.08 \\
\hline$\sigma$ & 0.76 & 0.33 & 0.20 & 0.19 & 1.00 & 1.55 & 0.58 & 1.30 & 0.79 & 2.28 & 0.31 & 0.24 & 0.13 & 0.04 & 0.18 & 0.32 & 0.31 & 0.28 & 0.41 & 0.06 & 2.08 & 0.11 \\
\hline$\mu$ & 0.67 & 0.76 & 0.51 & 0.46 & 0.64 & 0.23 & 0.22 & 0.29 & 0.01 & 1.33 & 0.31 & 0.12 & 0.13 & -0.03 & $\begin{array}{l}0.17 \\
\end{array}$ & 0.23 & 0.17 & 0.06 & -0.17 & 0.02 & 2.02 & -0.10 \\
\hline$\sigma$ & 0.24 & 0.31 & 0.26 & 0.19 & 0.38 & 0.06 & 0.36 & 0.90 & 1.06 & 4.13 & 0.50 & 0.21 & 0.20 & 0.04 & 0.27 & 0.35 & 0.18 & 0.16 & 0.29 & 0.05 & 3.77 & 0.14 \\
\hline$\mu$ & 1.20 & 1.17 & 0.49 & 0.44 & 0.64 & 1.04 & 0.21 & 0.05 & -0.12 & 0.14 & 0.47 & -0.19 & 0.17 & -0.04 & 0.24 & -0.28 & 0.18 & 0.49 & -0.21 & 0.02 & 7.00 & -0.12 \\
\hline$\sigma$ & 0.89 & 0.48 & 0.34 & 0.24 & 0.38 & 1.18 & 0.66 & 1.68 & 0.79 & 1.80 & 0.82 & 0.36 & 0.30 & 0.06 & 0.41 & 0.43 & 0.92 & $\begin{array}{l}0.78 \\
\end{array}$ & 0.51 & 0.09 & 13.76 & 0.19 \\
\hline$\mu$ & 0.62 & 2.12 & 0.68 & 0.48 & 0.38 & 0.34 & 0.14 & 0.11 & 0.18 & 0.88 & 0.32 & -0.10 & 0.11 & -0.03 & 0.16 & -0.11 & 0.02 & 0.22 & -0.13 & 0.03 & 7.50 & -0.07 \\
\hline$\sigma$ & 0.34 & 3.07 & 0.31 & 0.20 & 0.25 & 0.23 & 0.48 & 0.89 & 0.53 & 1.86 & 0.75 & 0.30 & 0.29 & 0.05 & 0.38 & 0.48 & 0.31 & 0.53 & 0.41 & 0.06 & 15.18 & 0.20 \\
\hline \multirow[b]{2}{*}{]$^{0}$} & 0.67 & 0.73 & 0.46 & 0.44 & 0.36 & 0.32 & 0.23 & 0.43 & -0.45 & 0.49 & 0.40 & -0.15 & 0.17 & -0.02 & 0.21 & 0.29 & 0.32 & 0.22 & 0.19 & -0.00 & 3.69 & -0.12 \\
\hline & 0.25 & 0.29 & 0.33 & 0.26 & 0.14 & 0.14 & 0.39 & 0.63 & 0.52 & 0.68 & 0.55 & 0.26 & 0.20 & 0.06 & 0.29 & 0.36 & 0.19 & 0.44 & 0.33 & 0.02 & 6.90 & 0.15 \\
\hline
\end{tabular}

Table 19: Frequency domain parameters SESSION 4 - SESSION 1 difference for EO EAE therapy 
$\underline{\text { Time domain parameters }}$

\begin{tabular}{|c|c|c|c|c|c|c|c|c|c|c|c|c|c|c|c|c|c|c|c|c|c|}
\hline Channel & Mean & MeanAbs & Median & Max & $\min$ & Peak & SSI & IEEG & $\mathrm{WL}$ & MMAV1 & \begin{tabular}{|l|} 
MMAV2 \\
\end{tabular} & STD DEV & VAR & ROOT & RMS & CREST & SKEWNESS & KURTOSIS & CLEARANCE & SHAPE & IMPULSE \\
\hline \multirow{2}{*}{1} & 12.41 & 0.16 & $-\quad 8.64$ & 0.05 & -0.27 & -0.14 & 0.03 & -0.16 & -0.14 & -0.18 & -0.28 & -0.14 & 0.02 & -0.16 & -0.14 & 0.11 & 4.76 & 0.24 & 0.13 & 0.01 & 0.12 \\
\hline & 22.65 & 0.61 & 15.58 & 0.93 & 0.54 & 0.60 & 0.95 & 0.61 & 0.16 & 0.60 & 0.52 & 0.62 & 0.95 & 0.61 & 0.62 & 0.24 & 8.58 & 0.53 & 0.23 & 0.03 & 0.23 \\
\hline \multirow[b]{2}{*}{2} & 6.18 & 0.13 & 11.73 & -0.15 & -0.16 & -0.13 & 1.16 & 0.13 & 0.10 & 0.19 & 0.26 & 0.12 & 1.13 & 0.13 & 0.13 & 0.01 & 54.94 & 0.14 & 0.10 & 0.03 & 0.06 \\
\hline & 11.96 & 1.11 & 21.98 & 0.59 & 0.53 & 0.61 & 3.29 & 1.11 & 0.20 & 1.21 & 1.36 & 1.08 & 3.25 & 1.11 & 1.09 & 0.36 & 94.36 & 0.32 & 0.52 & 0.08 & 0.45 \\
\hline & 23.05 & 0.64 & 7.31 & 0.19 & 0.48 & 0.23 & 3.24 & 0.64 & 0.28 & 0.71 & 0.73 & 0.60 & 3.24 & 0.66 & 0.61 & -0.04 & 1.66 & 0.06 & 0.09 & 0.02 & 0.07 \\
\hline & 36.47 & 1.54 & 14.33 & 0.70 & 1.06 & 0.75 & 6.55 & 1.54 & 0.39 & 1.66 & 1.60 & 1.49 & 6.54 & 1.56 & 1.49 & 0.28 & 0.68 & 0.12 & 0.25 & 0.03 & 0.26 \\
\hline \multirow{2}{*}{4} & 13.69 & 0.23 & 89.06 & 0.10 & 0.08 & 0.05 & 0.75 & 0.23 & 0.09 & 0.24 & 0.35 & 0.18 & 0.65 & 0.24 & 0.20 & 0.14 & 7.37 & 0.07 & 0.20 & 0.04 & 0.18 \\
\hline & 21.76 & 0.62 & 189.54 & 0.61 & 0.48 & 0.60 & 1.22 & 0.62 & 0.08 & 0.62 & 0.48 & 0.59 & 1.10 & 0.62 & 0.64 & 0.08 & 15.91 & 0.27 & 0.12 & 0.07 & 0.10 \\
\hline \multirow{2}{*}{5} & 0.49 & 0.30 & 0.01 & -0.39 & -0.26 & -0.33 & -0.46 & -0.30 & 0.03 & 0.30 & 0.35 & 0.30 & -0.46 & -0.30 & -0.31 & 0.01 & 15.95 & 0.03 & 0.02 & 0.00 & 0.01 \\
\hline & 1.83 & 0.28 & 2.01 & 0.18 & 0.31 & 0.21 & 0.33 & 0.28 & 0.10 & 0.28 & 0.33 & 0.26 & 0.32 & 0.29 & 0.27 & 0.14 & 30.46 & 0.04 & 0.19 & 0.03 & 0.17 \\
\hline \multirow{2}{*}{6} & 0.83 & 0.31 & 1.04 & -0.39 & -0.24 & -0.32 & -0.39 & -0.31 & 0.04 & 0.30 & 0.28 & 0.32 & -0.39 & -0.31 & -0.32 & 0.05 & 2.08 & 0.13 & 0.03 & 0.01 & 0.04 \\
\hline & 0.44 & 0.44 & 0.31 & 0.37 & 0.50 & 0.44 & 0.50 & 0.44 & 0.11 & 0.44 & 0.46 & 0.44 & 0.49 & 0.44 & 0.44 & 0.13 & 1.65 & 0.15 & 0.15 & 0.02 & 0.14 \\
\hline & 0.11 & 0.05 & 0.30 & 0.11 & -0.05 & -0.04 & 0.63 & 0.05 & 0.06 & 0.05 & 0.05 & 57 & 0.63 & 0.05 & 0.07 & -0.0 & 5.54 & 1.10 & 10 & 0.02 & 0.09 \\
\hline & 2.56 & 0.75 & 5.43 & 0.60 & 0.80 & 0.79 & 2.06 & 0.75 & 0.07 & 0.73 & 0.56 & 0.81 & 2.05 & 0.72 & 0.81 & 0.14 & 7.86 & 0.32 & 0.15 & 0.06 & 0.14 \\
\hline \multirow{2}{*}{8} & 0.48 & 0.16 & 0.69 & 0.03 & 0.11 & 0.02 & 0.72 & 0.16 & 0.45 & 0.14 & 0.08 & 0.18 & 0.74 & 0.15 & 0.16 & -0.11 & 0.17 & 0.25 & 0.08 & 0.01 & 0.10 \\
\hline & 0.60 & 0.72 & 2.49 & 0.58 & 0.54 & 0.62 & 1.76 & 0.72 & 0.54 & 0.71 & 0.70 & 0.68 & 1.73 & 0.73 & 0.71 & 0.01 & 3.31 & 0.31 & 0.08 & 0.03 & 0.04 \\
\hline \multirow{2}{*}{ s } & 2.88 & 0.27 & 1.04 & -0.28 & -0.36 & -0.33 & -0.40 & -0.27 & 0.12 & 0.25 & 0.12 & 0.29 & -0.41 & -0.25 & -0.28 & -0.03 & 0.35 & 0.12 & 0.10 & 0.04 & 0.07 \\
\hline & 2.31 & 0.32 & 0.41 & 0.28 & 0.19 & 0.27 & 0.45 & 0.32 & 0.18 & 0.32 & 0.29 & 0.34 & 0.45 & 0.31 & 0.33 & 0.13 & 0.90 & 0.15 & 0.04 & 0.05 & 0.08 \\
\hline \multirow{2}{*}{10} & 0.87 & 0.32 & 1.16 & -0.40 & -0.21 & -0.34 & -0.55 & -0.32 & 0.14 & 0.33 & 0.28 & 0.32 & -0.53 & -0.31 & -0.33 & -0.01 & 1.44 & 0.22 & 0.04 & 0.02 & 0.03 \\
\hline & 0.23 & 0.12 & 0.37 & 0.12 & 0.08 & 0.14 & 0.17 & 0.12 & 0.25 & 0.13 & 0.34 & 0.13 & 0.18 & 0.13 & 0.12 & 0.14 & 0.74 & 0.22 & 0.21 & 0.06 & 0.18 \\
\hline \multirow{2}{*}{11} & 3.40 & 0.41 & 3.79 & 0.08 & 0.17 & 0.12 & 2.27 & 0.41 & 0.08 & 0.51 & 0.56 & 0.37 & 2.27 & 0.43 & 0.37 & -0.03 & 3.45 & 0.06 & 0.06 & 0.02 & 0.05 \\
\hline & 5.56 & 1.43 & 7.01 & 0.93 & 0.94 & 0.93 & 5.15 & 1.43 & 0.26 & 1.64 & 1.84 & 1.36 & 5.15 & 1.46 & 1.36 & 0.27 & 3.82 & 0.19 & 0.26 & 0.03 & 0.26 \\
\hline \multirow{2}{*}{12} & 2.71 & 0.17 & 4.31 & -0.15 & -0.01 & -0.16 & -0.04 & -0.17 & 0.20 & 0.17 & 0.19 & 0.16 & -0.03 & -0.17 & -0.16 & 0.22 & 4.45 & 0.01 & 0.35 & 0.04 & 0.29 \\
\hline & 4.15 & 0.59 & 7.09 & 0.43 & 0.74 & 0.49 & 1.00 & 0.59 & 0.62 & 0.59 & 0.62 & 0.58 & 1.03 & 0.60 & 0.59 & 0.46 & 6.38 & 0.09 & 0.72 & 0.08 & 0.60 \\
\hline \multirow{2}{*}{13} & 137.65 & 0.15 & 1.92 & -0.04 & -0.04 & 0.00 & -0.09 & -0.15 & 0.02 & 0.14 & 0.07 & 0.15 & -0.08 & -0.15 & -0.15 & 0.26 & 0.59 & 0.07 & 0.35 & 0.03 & 0.31 \\
\hline & 273.58 & 0.52 & 2.07 & 0.63 & 0.46 & 0.60 & 0.70 & 0.52 & 0.09 & 0.53 & 0.64 & 0.51 & 0.70 & 0.52 & 0.51 & 0.24 & 0.74 & 0.06 & 0.41 & 0.06 & 0.33 \\
\hline \multirow{2}{*}{14} & 0.42 & 0.29 & 0.87 & -0.18 & -0.39 & -0.26 & -0.20 & -0.29 & 0.09 & 0.30 & 0.39 & 0.28 & -0.20 & -0.28 & -0.29 & 0.17 & 1.59 & 0.04 & 0.06 & 0.04 & 0.11 \\
\hline & 1.15 & 0.62 & 0.44 & 0.74 & 0.44 & 0.64 & 1.07 & 0.62 & 0.56 & 0.60 & 0.48 & 0.62 & 1.06 & 0.61 & 0.63 & 0.30 & 1.38 & 0.11 & 0.07 & 0.08 & 0.18 \\
\hline \multirow{2}{*}{15} & 4.27 & 0.28 & 0.39 & -0.35 & -0.22 & -0.32 & -0.27 & -0.28 & -0.09 & 0.29 & 0.33 & 0. & -0.29 & -0.27 & -0.29 & -0.0 & 0.75 & 0.12 & 10 & 0.06 & 0.06 \\
\hline & 5.25 & 0.54 & 3.27 & 0.49 & 0.59 & 0.48 & 0.70 & 0.54 & 0.21 & 0.54 & 0.49 & 0. & 0.69 & 0.54 & 0.54 & 0.10 & 3.21 & 0.23 & 0.17 & 0.10 & 0.13 \\
\hline \multirow{2}{*}{$16 \frac{\mu}{\sigma}$} & 8.24 & 0.27 & 0.62 & -0.26 & -0.10 & -0.18 & -0.28 & -0.27 & 0.02 & 0.28 & 0.34 & 0.26 & -0.27 & -0.27 & -0.26 & 0.18 & 3.00 & 0.05 & 0.26 & 0.03 & 0.22 \\
\hline & 14.62 & 0.48 & 0.85 & 0.51 & 0.59 & 0.53 & 0.59 & 0.48 & 0.22 & 0.46 & 0.40 & 0.48 & 0.59 & 0.47 & 0.48 & 0.22 & 3.09 & 0.19 & 0.34 & 0.05 & 0.28 \\
\hline
\end{tabular}

Table 20: Time domain parameters SESSION 4 - SESSION 1 difference for EO EAE therapy 
- $\quad$ RS therapy (data from 1 patient)

Frequency domain parameters

\begin{tabular}{|c|c|c|c|c|c|c|c|c|c|c|c|c|c|c|c|c|c|c|c|c|c|c|}
\hline Channel & PSDtotal & PSD- $\delta$ & PSD- $\theta$ & PSD- $\alpha$ & PSD- $\beta$ & PSD- $-\gamma$ & 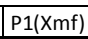 & P2 & P3 & P4 & P5(Xfc) & $\mathrm{P} 6$ & P7(Xrmsf) & P8 & P9 & $\mathrm{P} 10$ & P11 & $\mathrm{P} 12$ & P13 & P14 & $\mathrm{MDF}$ & \begin{tabular}{|l|} 
PSDef \\
\end{tabular} \\
\hline 1 & 1.48 & 0.79 & 0.39 & 2.21 & 1.99 & 2.55 & 0.57 & 0.01 & 0.86 & 0.99 & \begin{tabular}{|l|}
0.16 \\
\end{tabular} & 0.17 & 0.08 & -0.01 & 0.09 & 0.00 & 0.47 & -0.38 & 0.40 & -0.07 & 0.22 & -0.07 \\
\hline 2 & 1.12 & 0.74 & 0.42 & 1.73 & 1.50 & 1.51 & 0.43 & 0.19 & 0.78 & 0.97 & 0.06 & 0.11 & 0.01 & -0.02 & 0.04 & 0.06 & 0.20 & -0.27 & 0.30 & -0.07 & 0.07 & -0.04 \\
\hline 3 & 0.67 & 0.91 & 0.48 & 0.61 & 0.51 & 0.48 & 0.32 & 0.74 & 0.70 & 0.93 & 0.06 & -0.22 & 0.02 & -0.01 & 0.03 & 0.26 & 0.31 & 0.47 & -0.25 & -0.05 & 0.06 & -0.04 \\
\hline 4 & 0.67 & 0.38 & 0.60 & 1.01 & 0.46 & 0.81 & 0.37 & 0.25 & 0.35 & 0.38 & -0.27 & -0.23 & 0.19 & -0.02 & -0.17 & 0.05 & 3.34 & 1.17 & -0.30 & -0.03 & 0.45 & 0.11 \\
\hline 5 & 0.63 & 0.65 & 0.25 & 0.50 & 0.34 & 0.65 & 0.29 & 0.53 & 0.66 & 0.93 & -0.15 & -0.24 & 0.13 & -0.02 & -0.10 & 0.11 & 1.21 & 1.00 & -0.24 & -0.10 & 0.27 & 0.02 \\
\hline 6 & 0.61 & 0.79 & 0.39 & 0.29 & 0.22 & 0.73 & 0.36 & 0.58 & 0.54 & 0.86 & -0.15 & -0.27 & 0.12 & -0.02 & -0.11 & 0.15 & 1.38 & 1.23 & -0.30 & -0.09 & 0.29 & 0.03 \\
\hline 7 & 0.67 & 1.62 & 0.21 & 0.54 & 0.33 & 0.74 & 0.29 & 0.04 & 1.15 & 4.77 & -0.20 & -0.15 & 0.13 & \begin{tabular}{|l|}
0.00 \\
\end{tabular} & -0.13 & 0.07 & 1.49 & 0.69 & -0.22 & 0.01 & 0.38 & 0.09 \\
\hline 8 & 0.96 & 0.88 & 0.34 & 0.49 & 0.95 & 0.99 & 0.83 & 0.95 & 0.10 & 0.62 & -0.35 & -0.58 & 0.24 & -0.02 & -0.22 & 0.35 & 22.76 & 6.25 & -0.74 & 0.04 & 0.55 & 0.17 \\
\hline 9 & 0.86 & 1.67 & 0.65 & 1.45 & 0.49 & 0.85 & 0.35 & 0.13 & 0.39 & 0.14 & -0.34 & -0.23 & 0.24 & -0.01 & -0.23 & 0.17 & 3.76 & 1.45 & -0.29 & -0.05 & 0.55 & 0.16 \\
\hline 10 & 0.66 & 0.68 & 0.68 & 1.47 & 0.32 & 0.77 & 0.24 & 0.21 & 0.15 & 0.48 & -0.30 & -0.18 & 0.21 & -0.01 & -0.20 & 0.17 & 2.23 & 1.08 & -0.20 & -0.06 & 0.49 & 0.13 \\
\hline 11 & 0.90 & 0.79 & 0.45 & 0.30 & 0.84 & 0.96 & 0.72 & 0.87 & 0.02 & 0.35 & -0.29 & -0.50 & 0.21 & -0.05 & -0.17 & 0.29 & 14.40 & 3.44 & -0.62 & -0.04 & 0.44 & 0.10 \\
\hline 12 & 0.97 & 0.72 & 0.72 & 0.68 & 0.97 & 0.99 & 0.86 & 0.95 & 1.74 & 7.78 & -0.32 & -0.60 & 0.22 & -0.03 & \begin{tabular}{|l|}
-0.19 \\
\end{tabular} & 0.41 & 12.98 & 6.60 & -0.76 & 0.05 & 0.50 & 0.16 \\
\hline 13 & 0.37 & 0.35 & 0.28 & 0.50 & 0.78 & 0.76 & 0.32 & 0.36 & 0.21 & 0.36 & -0.23 & -0.25 & 0.17 & -0.01 & -0.16 & 0.02 & 1.42 & 1.29 & -0.27 & -0.09 & 0.38 & 0.08 \\
\hline 14 & 0.75 & 0.48 & 0.34 & 0.42 & 0.78 & 0.95 & 0.64 & 0.64 & 1.79 & 5.75 & -0.32 & -0.43 & 0.22 & -0.03 & -0.20 & 0.16 & 6.42 & 3.09 & -0.55 & -0.04 & 0.50 & 0.14 \\
\hline 15 & 0.96 & 1.00 & 0.88 & 0.65 & 0.83 & 0.93 & 0.73 & 0.97 & 0.82 & 0.97 & 0.01 & -0.51 & 0.01 & \begin{tabular}{|l|l}
0.00 \\
\end{tabular} & -0.02 & 0.51 & 2.25 & 2.90 & -0.62 & -0.05 & 0.11 & -0.02 \\
\hline 16 & 0.86 & 0.92 & 0.24 & 0.88 & 0.42 & 0.87 & 0.43 & 0.27 & 3.80 & 23.18 & -0.35 & -0.29 & 0.25 & -0.04 & -0.23 & 0.11 & 7.19 & 2.90 & -0.36 & -0.06 & 0.55 & 0.16 \\
\hline
\end{tabular}

Table 21: Frequency domain parameters SESSION 4 - SESSION 1 difference for EO RS therapy

Time domain parameters

\begin{tabular}{|c|c|c|c|c|c|c|c|c|c|c|c|c|c|c|c|c|c|c|c|c|c|}
\hline Channel & Mean & MeanAbs & Median & Max & $\min$ & Peak & SSI & IEEG & WL & MMAV1 & MMAV2 & STD DEV & VAR & ROOT & RMS & CREST & SKEWNESS & KURTOSIS & CLEARANCE & SHAPE & IMPULSE \\
\hline 1 & 0.87 & 0.27 & 1.97 & 0.27 & 0.32 & 0.27 & 0.63 & 0.27 & 0.83 & 0.29 & 0.33 & 0.28 & 0.63 & 0.27 & 0.28 & -0.00 & 1.19 & 0.02 & 0.00 & 0.00 & 0.00 \\
\hline 2 & 1.12 & 0.34 & 1.05 & 0.48 & 0.26 & 0.40 & 0.75 & 0.34 & 0.19 & 0.32 & 0.28 & 0.32 & 0.75 & 0.36 & 0.32 & 0.06 & 1.25 & 0.05 & 0.03 & -0.01 & 0.04 \\
\hline 3 & 0.99 & 0.29 & 1.07 & -0.37 & -0.21 & -0.33 & -0.49 & -0.29 & -0.21 & -0.29 & -0.25 & -0.29 & -0.49 & -0.29 & -0.29 & -0.06 & 1.24 & 0.05 & 0.06 & 0.00 & 0.06 \\
\hline 4 & 1.10 & 0.46 & 1.44 & -0.42 & -0.52 & -0.46 & -0.72 & -0.46 & -0.56 & -0.48 & -0.53 & -0.47 & -0.72 & -0.46 & -0.47 & 0.02 & 2.30 & 0.01 & 0.00 & -0.01 & 0.01 \\
\hline 5 & 1.03 & 0.09 & 1.07 & 0.05 & -0.14 & 0.05 & -0.17 & -0.09 & -0.40 & -0.11 & -0.15 & -0.07 & -0.13 & -0.10 & -0.09 & 0.15 & 2.14 & 0.02 & 0.17 & 0.01 & 0.16 \\
\hline 6 & 1.31 & 0.48 & 5.55 & -0.55 & -0.48 & -0.55 & -0.74 & -0.48 & -0.47 & -0.47 & -0.45 & 0.49 & -0.74 & -0.47 & -0.49 & -0.11 & 0.93 & 0.11 & 0.14 & -0.02 & 0.13 \\
\hline 7 & 1.74 & 0.32 & 0.70 & -0.24 & -0.20 & -0.20 & -0.53 & -0.32 & -0.49 & -0.31 & -0.30 & -0.31 & -0.53 & -0.32 & -0.31 & 0.17 & 0.23 & 0.01 & 0.18 & 0.01 & 0.17 \\
\hline 8 & \begin{tabular}{|l|}
-10.01 \\
\end{tabular} & 0.75 & 5.58 & -0.75 & -0.72 & -0.72 & -0.94 & -0.75 & -0.89 & -0.75 & -0.75 & -0.76 & -0.94 & -0.75 & -0.75 & 0.15 & 26.36 & 0.24 & 0.15 & -0.00 & 0.15 \\
\hline 9 & 5.44 & 0.06 & 3.67 & 0.20 & 0.18 & 0.18 & -0.09 & -0.06 & -0.55 & -0.09 & -0.19 & -0.05 & -0.09 & -0.07 & -0.04 & 0.23 & 9.18 & 0.23 & 0.27 & 0.02 & 0.25 \\
\hline 10 & 1.38 & 0.14 & 1.05 & -0.12 & -0.30 & -0.12 & -0.27 & -0.14 & -0.46 & -0.16 & -0.31 & -0.15 & -0.27 & -0.14 & -0.15 & 0.04 & 0.19 & 0.08 & 0.03 & -0.01 & 0.03 \\
\hline 11 & 0.92 & 0.67 & 0.77 & -0.66 & -0.57 & -0.63 & -0.89 & -0.67 & -0.79 & \begin{tabular}{|l}
-0.67 \\
\end{tabular} & -0.66 & -0.67 & -0.89 & -0.67 & -0.67 & 0.11 & 5.46 & 0.12 & 0.12 & 0.01 & 0.12 \\
\hline 12 & 1.16 & 0.78 & 1.24 & -0.76 & -0.85 & -0.81 & -0.95 & -0.78 & -0.87 & -0.79 & -0.82 & 0.78 & -0.95 & -0.78 & -0.78 & -0.15 & 2.07 & 0.03 & 0.14 & 0.00 & 0.15 \\
\hline 13 & 2.65 & 0.28 & 8.13 & -0.26 & -0.44 & -0.28 & -0.46 & -0.28 & -0.49 & -0.29 & -0.37 & -0.28 & -0.48 & -0.29 & -0.27 & -0.02 & 2.11 & 0.05 & 0.01 & 0.02 & 0.00 \\
\hline 14 & 3.19 & 0.46 & 154.50 & -0.50 & -0.36 & -0.41 & -0.70 & -0.46 & -0.75 & -0.46 & -0.47 & -0.46 & -0.71 & -0.46 & -0.45 & 0.08 & 18.05 & 0.12 & 0.10 & 0.01 & 0.09 \\
\hline 15 & 1.02 & 0.69 & $\begin{array}{r}-\quad 1.04 \\
\end{array}$ & -0.64 & -0.73 & -0.64 & -0.91 & -0.69 & -0.71 & \begin{tabular}{|l|}
-0.70 \\
\end{tabular} & -0.72 & -0.69 & -0.90 & -0.69 & -0.69 & 0.16 & 0.85 & 0.06 & 0.15 & -0.01 & 0.15 \\
\hline 16 & 4.03 & 0.26 & 6.30 & -0.05 & -0.43 & -0.11 & -0.42 & -0.26 & -0.67 & -0.28 & -0.35 & -0.24 & -0.42 & -0.27 & -0.24 & 0.17 & 14.54 & 0.27 & 0.22 & 0.03 & 0.20 \\
\hline
\end{tabular}

Table 22: Time domain parameters SESSION 4 - SESSION 1 difference for EO RS therapy 
ANNEX X: SESSION 4 - SESSION 1 DIFFERENCE OF TIME AND TREQUENCY DOMAIN PARAMETERS FROM EACH ACOUSTIC THERAPY FOR EC: SELECTION OF PARAMETERS FOR FRONTAL AND TEMPORAL LOBE

\begin{tabular}{|c|c|c|c|c|c|c|c|c|c|c|c|c|c|c|c|}
\hline & & \multicolumn{4}{|c|}{ Time domain parameters selection } & \multicolumn{10}{|c|}{ Frequency domain parameters selection } \\
\hline Binaural & & Mean & Peak & RMS & KURTOSIS & PSDtotal & PSD- $\delta$ & PSD- $\theta$ & PSD- $\alpha$ & PSD- $\beta$ & PSD- $\gamma$ & P1(Xmf) & $\mathrm{P} 4$ & $\mathrm{P5}(\mathrm{Xfc})$ & P13 \\
\hline \multirow{2}{*}{1} & $\mu$ & - 129.27 & 1.73 & 1.13 & 2.00 & 5.05 & 20.47 & 0.84 & 1.51 & 0.29 & 0.22 & 0.30 & 5.04 & 0.22 & 0.21 \\
\hline & $\sigma$ & 186.61 & 2.41 & 1.91 & 0.01 & 5.11 & 12.31 & 0.14 & 0.89 & 0.05 & 0.01 & 0.38 & 5.07 & 0.20 & 0.24 \\
\hline \multirow{2}{*}{2} & $\mu$ & $-\quad 39.43$ & 2.57 & 1.37 & 1.91 & 10.36 & 31.20 & 0.86 & 1.18 & 0.25 & 0.36 & 0.22 & 7.37 & 0.26 & 0.14 \\
\hline & $\sigma$ & 50.38 & 3.37 & 2.19 & 0.15 & 14.01 & 41.42 & 0.59 & 0.81 & 0.01 & 0.15 & 0.53 & 9.54 & 0.24 & 0.36 \\
\hline \multirow{2}{*}{3} & $\mu$ & -168.56 & 0.73 & 0.85 & 0.57 & 11.06 & 46.07 & 1.05 & 1.00 & 0.54 & 0.65 & 0.05 & 10.67 & 0.34 & 0.03 \\
\hline & $\sigma$ & 55.48 & 1.55 & 1.62 & 0.06 & 12.23 & 58.73 & 0.75 & 0.35 & 0.06 & 0.06 & 0.09 & 3.04 & 0.12 & 0.07 \\
\hline \multirow{2}{*}{7} & $\mu$ & 40.80 & 0.76 & 0.34 & 1.46 & 9.14 & 19.30 & 1.54 & 1.94 & 0.56 & 0.78 & 0.14 & 18.58 & 0.38 & 0.12 \\
\hline & $\sigma$ & 60.84 & 0.80 & 0.60 & 0.03 & 11.17 & 19.40 & 1.98 & 2.06 & 0.11 & 0.09 & 0.34 & 14.25 & 0.07 & 0.25 \\
\hline EAE & & Mean & Peak & RMS & KURTOSIS & PSDtotal & PSD- $\delta$ & PSD- $\theta$ & PSD- $\alpha$ & PSD- $\beta$ & PSD- $\gamma$ & P1(Xmf) & P4 & P5(Xfc) & P13 \\
\hline \multirow{2}{*}{1} & $\mu$ & $\begin{array}{l}-\quad 2.29 \\
\end{array}$ & 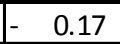 & 0.01 & $-\quad 0.10$ & 2.33 & 2.50 & 0.62 & 0.57 & 0.61 & 0.53 & -0.33 & 12.84 & 0.38 & $\begin{array}{ll}- & 0.28 \\
\end{array}$ \\
\hline & $\sigma$ & 2.47 & 0.91 & 1.15 & 0.19 & 2.00 & 1.85 & 0.36 & 0.30 & 0.24 & 0.22 & 0.49 & 15.64 & 0.85 & 0.40 \\
\hline \multirow{2}{*}{2} & $\mu$ & 0.17 & 0.37 & 0.27 & 0.21 & 0.88 & 0.97 & 0.76 & 0.59 & 0.53 & 0.42 & 0.46 & 0.50 & 0.61 & 0.38 \\
\hline & $\sigma$ & 3.06 & 0.88 & 1.07 & 0.08 & 0.18 & 0.26 & 0.18 & 0.30 & 0.28 & 0.02 & 0.36 & 0.39 & 0.85 & 0.31 \\
\hline \multirow{2}{*}{3} & $\mu$ & 11.14 & 0.11 & 0.17 & 0.04 & 0.71 & 0.61 & 0.54 & 0.44 & 0.41 & 0.49 & 0.10 & 0.29 & 0.18 & 0.10 \\
\hline & $\sigma$ & 19.93 & 0.56 & 0.61 & 0.26 & 0.25 & 0.38 & 0.33 & 0.37 & 0.30 & 0.21 & 0.31 & 0.49 & 0.27 & 0.24 \\
\hline \multirow{2}{*}{7} & $\mu$ & 14.91 & 0.17 & 0.10 & 0.15 & 1.39 & 2.41 & 0.68 & 0.57 & 0.43 & 0.45 & 0.29 & 2.33 & 0.16 & 0.26 \\
\hline & $\sigma$ & 25.53 & 0.94 & 1.08 & 0.25 & 0.87 & 3.05 & 0.31 & 0.35 & 0.35 & 0.34 & 0.51 & 5.49 & 0.59 & 0.42 \\
\hline TRT & & Mean & Peak & RMS & KURTOSIS & PSDtotal & PSD- $\delta$ & PSD- $\theta$ & PSD- $\alpha$ & PSD- $\beta$ & $\overline{\text { PSD- } \gamma}$ & $\mathrm{P} 1(\mathrm{Xmf})$ & P4 & $\mathrm{P5}(\mathrm{Xfc})$ & P13 \\
\hline \multirow{2}{*}{1} & $\mu$ & 7.28 & 0.09 & 0.27 & 0.11 & 1.60 & 3.91 & 0.59 & 0.50 & 0.32 & 0.53 & $\begin{array}{r}-0.19 \\
\end{array}$ & 4.44 & 0.30 & 0.16 \\
\hline & $\sigma$ & 13.94 & 0.88 & 1.24 & 0.23 & 2.18 & 7.89 & 0.35 & 0.25 & 0.12 & 0.38 & 0.39 & 10.54 & 0.50 & 0.30 \\
\hline \multirow{2}{*}{2} & $\mu$ & 1.14 & 0.32 & 0.31 & 0.11 & 1.11 & 1.43 & 0.59 & 0.42 & 0.56 & 1.01 & 0.23 & 0.39 & 0.39 & 0.18 \\
\hline & $\sigma$ & 1.17 & 0.42 & 0.45 & 0.35 & 0.80 & 1.63 & 0.22 & 0.17 & 0.40 & 1.23 & 0.38 & 1.56 & 0.59 & 0.28 \\
\hline \multirow{2}{*}{3} & $\mu$ & 3.35 & 0.29 & 0.64 & 0.22 & 1.94 & 3.61 & 0.85 & 0.72 & 0.68 & 0.47 & 0.01 & 0.81 & 0.04 & 0.02 \\
\hline & $\sigma$ & 9.14 & 1.40 & 1.90 & 0.24 & 2.43 & 5.14 & 0.64 & 0.44 & 0.83 & 0.29 & 0.53 & 1.19 & 0.44 & 0.38 \\
\hline \multirow{2}{*}{7} & $\mu$ & 4.98 & 0.34 & 0.53 & 0.10 & 2.40 & 5.34 & 0.82 & 0.67 & 0.33 & 0.30 & 0.02 & 1.85 & 0.05 & 0.04 \\
\hline & $\sigma$ & 4.48 & 0.74 & 0.93 & 0.30 & 3.93 & 8.83 & 0.47 & 0.32 & 0.19 & 0.17 & 0.40 & 3.08 & 0.46 & 0.30 \\
\hline RS & & Mean & Peak & RMS & KURTOSIS & PSDtotal & PSD- $\delta$ & PSD- $\theta$ & PSD- $\alpha$ & PSD- $\beta$ & PSD- $y$ & $\mathrm{P} 1(\mathrm{Xmf})$ & P4 & $\mathrm{P5}(\mathrm{Xfc})$ & $\mathrm{P} 13$ \\
\hline 1 & $\mu$ & $\begin{array}{ll}- & 1.15 \\
\end{array}$ & $\begin{array}{l}0.57 \\
\end{array}$ & 1.03 & 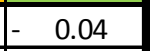 & 1.29 & \begin{tabular}{|l|}
0.34 \\
\end{tabular} & 0.54 & 1.58 & 1.62 & \begin{tabular}{|l|}
1.78 \\
\end{tabular} & 0.53 & 0.86 & 0.05 & 0.36 \\
\hline 2 & $\mu$ & 7.30 & 1.12 & 1.37 & 0.01 & 0.53 & 0.14 & 0.60 & 1.42 & 0.80 & 0.23 & 0.08 & 0.05 & 0.12 & 0.06 \\
\hline 3 & $\mu$ & 1.69 & 0.26 & 0.48 & 0.28 & 0.49 & 0.54 & 0.27 & 1.01 & 0.45 & 0.28 & 0.05 & 0.75 & 0.02 & 0.03 \\
\hline 7 & $\mu$ & 17.51 & 0.20 & 0.35 & 0.06 & 0.58 & 0.68 & 0.22 & 0.55 & 0.31 & 0.63 & 0.24 & 0.60 & 0.15 & 0.20 \\
\hline
\end{tabular}

Table 23: Difference between session 4 and session 1: time and frequency parameters selection for resting (EC) analysis 

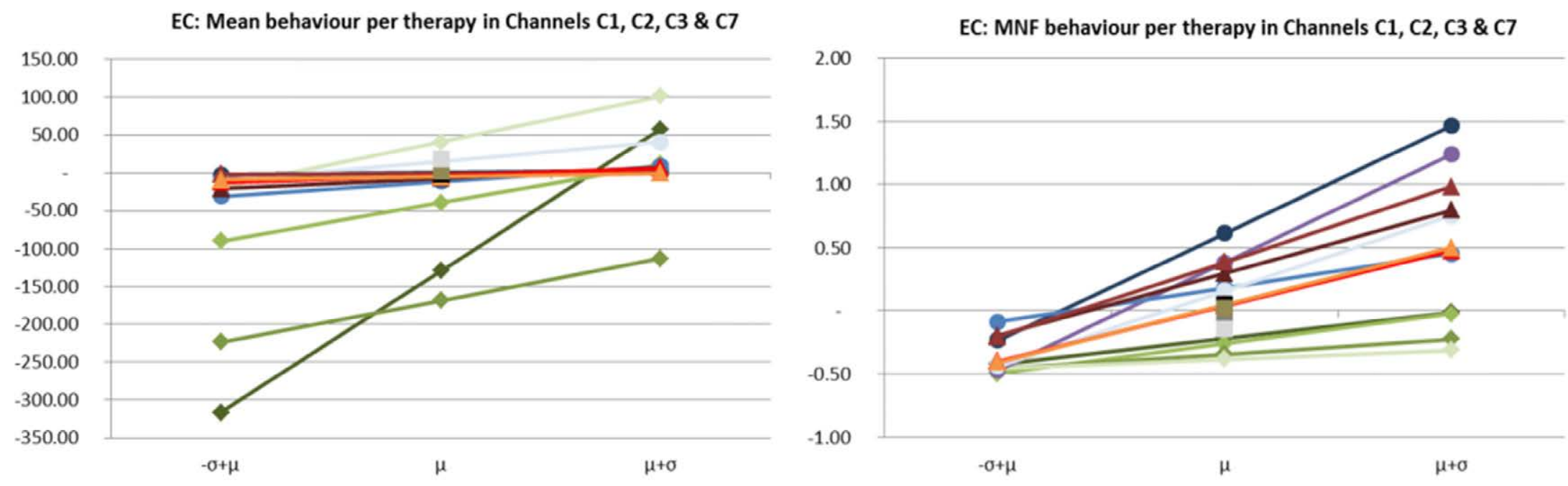

(a)

(b)

$$
\begin{aligned}
& \multimap \text { Binaural C1 } \longrightarrow \text { Binaural C2 } \multimap \text { Binaural C3 } \leadsto \text { Binaural C } \\
& \leadsto \text { EAEC1 }- \text { EAEC2 }- \text { EAEC3 } \\
& \text { —TRT C1 —TRT C2 —TRT C3 - } \\
& \rightarrow \text { RSC1 }-\mathrm{RSC2}-\mathrm{RSC3}-\mathrm{RSC7}
\end{aligned}
$$

Figure 28: EC analysis: Frontal and temporal lobe behavior per therapy shown by the difference between session 4 and session 1 of the Mean (a) and MNF (b) representation 


\section{ANNEX Y: SESSION 4 - SESSION 1 DIFFERENCE OF TIME AND TREQUENCY DOMAIN PARAMETERS FROM EACH ACOUSTIC THERAPY FOR EC}

In the following tables are found the Average $(\mu)$ and Standard Deviation $(\sigma)$ of the time and frequency domain parameters difference between session 4 and session 1 (relativized by Session 1) for the resting condition (Eyes Closed) of all patients per acoustic therapy.

- Binaural $f_{0}$ therapy (data from 2 patients)

Frequency domain parameters

\begin{tabular}{|c|c|c|c|c|c|c|c|c|c|c|c|c|c|c|c|c|c|c|c|c|c|c|}
\hline Channel & PSDtotal & PSD- $\delta$ & SD- $\theta$ & PSD- $\alpha$ & PSD- $\beta$ & PSD- $-\gamma$ & \begin{tabular}{|l|}
$\mathrm{P} 1(\mathrm{Xmf})$ \\
\end{tabular} & P2 & P3 & P4 & $\mathrm{P5}(\mathrm{Xfc})$ & P6 & P7(Xrmsf) & P8 & $\mathrm{P9}$ & P10 & P11 & P12 & P13 & P14 & MDF & $\begin{array}{l}\text { PSDef } \\
\end{array}$ \\
\hline$\mu$ & 5.05 & 20.47 & 0.84 & 1.51 & 0.29 & 0.22 & 0.30 & 9.53 & 1.65 & 5.04 & -0.22 & 0.16 & 0.13 & 0.01 & 0.13 & 0.55 & 0.72 & 0.05 & 0.21 & 0.02 & 0.39 & 0.14 \\
\hline$\mu$ & 10.36 & 31.20 & 0.86 & 1.18 & 0.25 & 0.36 & 0.22 & 14.41 & 84 & 7.37 & 0.26 & 0.07 & 0.16 & -0.01 & 0.16 & 0.58 & 1.16 & 0.14 & 0.14 & .01 & 0.46 & 0.16 \\
\hline 2 辰 & 14.01 & 41.42 & 0.59 & 0.81 & 0.01 & 0.15 & 0.53 & 20.29 & .02 & 9.54 & 0.24 & 0.18 & 0.15 & 0.01 & 0.14 & 0.75 & 0.53 & 0.11 & 0.36 & .05 & 0.44 & 0.17 \\
\hline$3 \longdiv { \sigma }$ & 12.23 & 58.73 & 0.75 & 0.35 & 0.06 & 0.06 & 0.09 & 17.26 & .35 & 3.04 & 0.12 & 0.07 & 0.08 & 0.01 & 0.07 & & 7.59 & 0.08 & 0.07 & & 0.17 & 0.10 \\
\hline$\mu$ & 9.32 & 45.77 & 1.91 & 2.36 & 0.41 & 0.54 & 0.15 & 14.54 & 5.48 & 32.80 & -0.36 & 0.08 & 0.22 & 0.01 & 0.23 & 0.69 & 1.84 & 0.30 & 0.10 & 0.01 & 0.64 & 0.22 \\
\hline$\sigma$ & 2.45 & 25.88 & 1.86 & 0.51 & 0.22 & 0.18 & 0.25 & 10.05 & 1.00 & 17.88 & 0.05 & 0.08 & 0.04 & 0.02 & 0.02 & 0.25 & 0.35 & 0.14 & 0.16 & 0.04 & 0.07 & 0.04 \\
\hline$\mu$ & 4.03 & 33.07 & 0.75 & 1.32 & 0.28 & 0.52 & 0.07 & 7.44 & 3.00 & 18.28 & -0.28 & 0.0 & 0.1 & 0.01 & 0.18 & & 1.42 & & 0.03 & & 0.51 & 0.16 \\
\hline & 4.35 & 47.47 & 0.07 & 0.85 & 0.12 & 0.06 & 0.17 & 8.6 & 2.33 & & 0.18 & 0.0 & & & 0. & & & & & & & 12 \\
\hline$\mu$ & 9.14 & 19.30 & 1.54 & 1.94 & 0.56 & 0.78 & 0.14 & 9.52 & 3.58 & 18.58 & -0.38 & 0.08 & 0.25 & -0.00 & 0.24 & 0.51 & 16.55 & 0.78 & 0.12 & 0.01 & 0.62 & 0.23 \\
\hline$\sigma$ & 11.17 & 19.40 & 1.98 & 2.06 & 0.11 & 0.09 & 0.34 & 12.78 & 0.74 & 14.25 & 0.07 & 0.14 & 0.04 & 0.02 & 0.05 & 0.40 & 26.63 & 0.27 & 0.25 & 0.0 & 0.15 & 0.08 \\
\hline$\mu$ & 21.23 & 94.41 & 1.30 & 1.38 & 0.64 & 0.71 & 0.10 & 27.66 & 2.43 & 4.29 & -0.39 & 0.02 & 0.22 & 0.02 & 0.24 & 0.63 & 39.09 & 0.79 & 0.08 & 0.0 & 0.68 & 0.27 \\
\hline $8 \frac{\sigma}{\sigma}$ & 27.89 & 130.04 & 1.22 & 0.86 & 0.29 & 0.31 & 0.67 & 38.22 & 0.74 & 1.92 & 0.04 & 0.41 & 0.00 & 0.05 & 0.04 & 0.79 & 59.83 & 1.07 & 0.53 & 0.02 & 0.16 & 0.08 \\
\hline & 3.50 & 13.33 & 1.16 & 1.74 & 0.41 & 0.45 & 0.04 & 3.62 & 2.82 & & -0.23 & 0.0 & & & 0.1 & & & & 0.0 & & & .13 \\
\hline$\sigma$ & 0.70 & 6.36 & 0.91 & 0.16 & 0.21 & 0.10 & 0.02 & 1.19 & 2.24 & 18.56 & 0.09 & 0.01 & 0.07 & 0.03 & 0.05 & 0.1 & 1.07 & 0.09 & 0.02 & 0.01 & 0.09 & 0.05 \\
\hline$\mu$ & 0.97 & 2.89 & 0.50 & 1.02 & 0.38 & 0.60 & 0.15 & 0.78 & 1.51 & 4.57 & -0.21 & 0.06 & 0.13 & 0.01 & 0.13 & 0.19 & 1.56 & 0.40 & -0.11 & 0.02 & 0.39 & 0.10 \\
\hline & 1.42 & & 0.83 & 0.88 & 1.57 & 0.48 & & & & & -0.19 & -0.05 & & & 0.11 & 0. & & & & & & 0.09 \\
\hline$\sigma$ & 0.72 & 0.81 & 0.67 & 0.39 & 1.53 & 0.20 & 0.25 & 1.29 & 0.66 & 3.81 & 0.02 & 0.10 & 0.01 & 0.03 & 0.03 & 0.1 & 0.50 & 0.06 & $\begin{array}{l}0.17 \\
\end{array}$ & 0.03 & 0.02 & 0.01 \\
\hline & 30.14 & 117.16 & 5.97 & 5.70 & 1.57 & 0.52 & 0.25 & 26.10 & 3.76 & 23.52 & -0.31 & 0.04 & 0.20 & -0.00 & -0.20 & 0.66 & 2.20 & 0.35 & 0.11 & 0.03 & 0.45 & 0.18 \\
\hline$\sigma$ & 41.70 & 165.13 & 7.95 & 6.75 & 1.53 & 0.21 & & 37.14 & & & 0.23 & 0.28 & & & & & & 0.24 & 0.51 & & 0.34 & 0.16 \\
\hline 4 & 18.30 & 38.79 & 4.06 & 5.47 & 1.00 & 0.56 & 0.49 & 18.18 & 1.44 & 3.29 & -0.19 & 0.19 & 0.12 & 0.01 & -0.13 & 0.61 & 0.63 & -0.07 & 0.33 & 0.02 & 0.33 & 0.13 \\
\hline$\sigma$ & 24.06 & 52.66 & 5.34 & 1.56 & 0.68 & 0.38 & 0.23 & 23.86 & 1.30 & 3.48 & 0.31 & 0.05 & 0.20 & 0.00 & 0.20 & 0.69 & 1.57 & 0.23 & 0.13 & 0.03 & 0.55 & 0.19 \\
\hline$\mu$ & 7.31 & 30.57 & 1.87 & 4.17 & 0.55 & 0.41 & 0.14 & 7.91 & 8.57 & 110.45 & -0.27 & 0.09 & 0.17 & 0.01 & 0.16 & 0.50 & 4.42 & -0.07 & 0.09 & 0.0 & 0.42 & 0.14 \\
\hline & 6.52 & 25.87 & 2.17 & 1.01 & 0.42 & 0.02 & 0.24 & 4.33 & 5.28 & 115.11 & 0.09 & 0.04 & 0.07 & 0.01 & 0.07 & 0.24 & 4.41 & 0.16 & 0.14 & 0.07 & 0.20 & 0.05 \\
\hline
\end{tabular}

Table 24: Frequency domain parameters SESSION 4 - SESSION 1 difference for EC Binaural $f_{0}$ therapy 


\section{Time domain parameters}

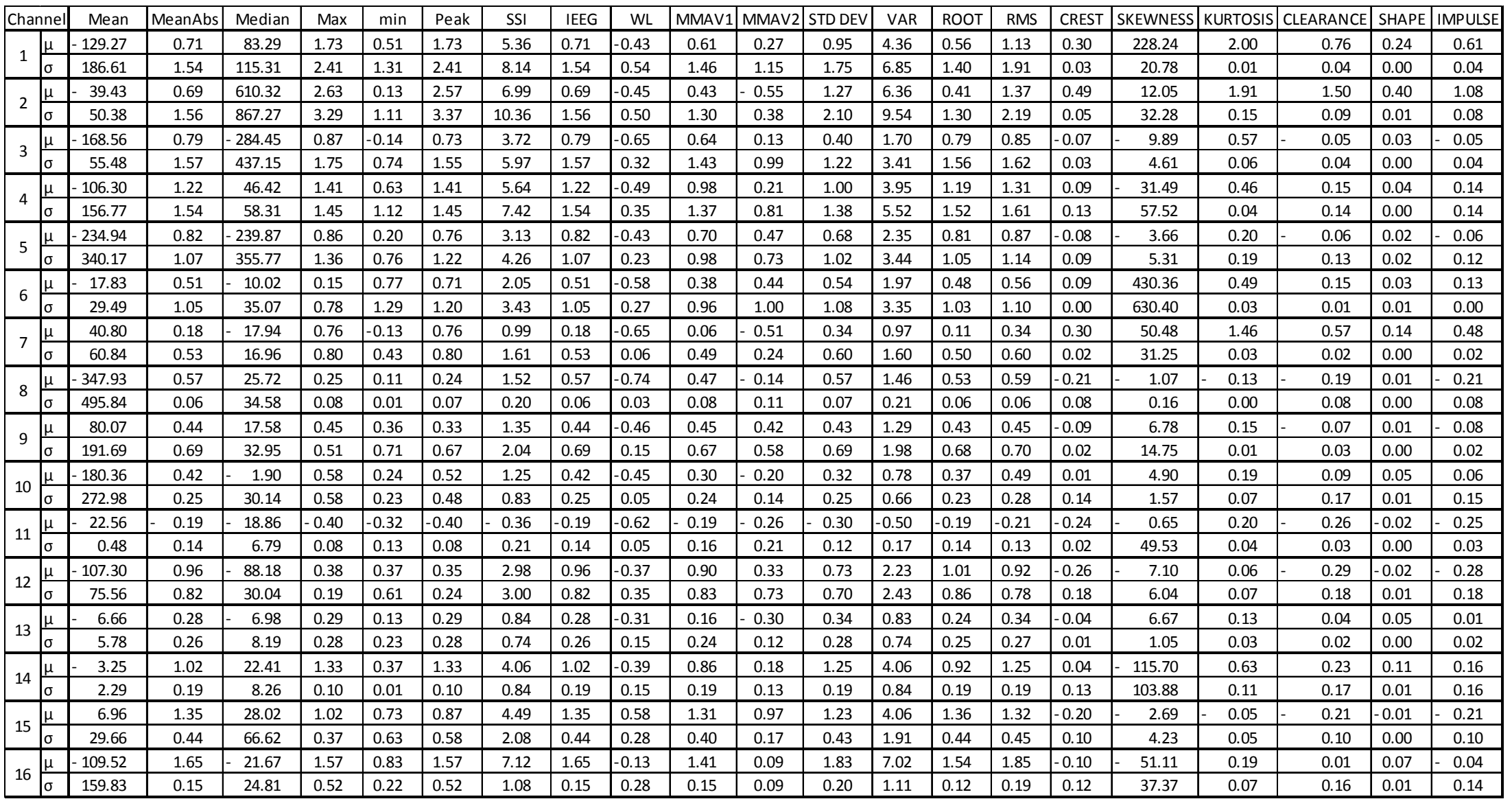

Table 25: Time domain parameters SESSION 4 - SESSION 1 difference for EC Binaural $f_{0}$ therapy 
- $\quad$ TRT therapy (data from 6 patients)

Frequency domain parameters

\begin{tabular}{|c|c|c|c|c|c|c|c|c|c|c|c|c|c|c|c|c|c|c|c|c|c|c|}
\hline \multirow{2}{*}{ Channel } & PSDtotal & $\begin{array}{l}\text { PSD- } \delta \\
\end{array}$ & PSD- $\theta$ & PSD- $\alpha$ & PSD- $\beta$ & PSD- -8 & $\mathrm{P} 1(\mathrm{Xmf})$ & $\mathrm{P} 2$ & P3 & P4 & $\mathrm{P} 5(\mathrm{Xfc})$ & P6 & $7($ Xrmsf) & P8 & $\mathrm{P} 9$ & $\mathrm{P} 10$ & P11 & P12 & $\mathrm{P} 13$ & P14 & $\mathrm{MDF}$ & PSDef \\
\hline & 1.60 & 3.91 & 0.59 & 0.50 & 0.32 & 0.53 & -0.19 & 0.88 & 0.57 & 4.44 & 0.30 & -0.11 & 0.12 & -0.03 & 0.16 & 0.19 & 0.08 & 0.10 & 0.16 & 0.02 & 1.94 & 0.09 \\
\hline$\sqrt[1]{\sigma}$ & 2.18 & 7.89 & 0.35 & 0.25 & 0.12 & 0.38 & 0.39 & 3.72 & 1.87 & 10.54 & 0.50 & 0.21 & 0.22 & 0.05 & 0.27 & 0.43 & 0.40 & 0.37 & 0.30 & 0.04 & 3.01 & 0.16 \\
\hline$\mu$ & 1.11 & 1.43 & 0.59 & 0.42 & 0.56 & 1.01 & 0.23 & 0.06 & 0.07 & 0.39 & 0.39 & -0.12 & 0.14 & -0.05 & 0.21 & -0.26 & 0.00 & 0.11 & -0.18 & 0.04 & 2.75 & 0.12 \\
\hline $2 \sqrt{\sigma}$ & 0.80 & 1.63 & 0.22 & 0.17 & 0.40 & 1.23 & 0.38 & 1.80 & 0.66 & 1.56 & 0.59 & 0.19 & 0.25 & 0.05 & 0.31 & 0.36 & 0.56 & 0.50 & 0.28 & 0.10 & 4.72 & 0.17 \\
\hline$\mu$ & 1.94 & 3.61 & 0.85 & 0.72 & 0.68 & 0.47 & 0.01 & 1.24 & 0.31 & 0.81 & 0.04 & 0.03 & 0.01 & -0.02 & 0.02 & 0.06 & 0.26 & 0.20 & -0.02 & 0.01 & 0.71 & 0.01 \\
\hline$\sqrt[3]{\sigma}$ & 2.43 & 5.14 & 0.64 & 0.44 & 0.83 & 0.29 & 0.53 & 2.44 & 0.55 & 1.19 & 0.44 & 0.26 & 0.17 & 0.07 & 0.27 & 0.43 & 0.50 & 0.43 & 0.38 & 0.06 & 2.38 & 0.16 \\
\hline$\mu$ & 0.54 & 0.64 & 0.36 & 0.38 & 0.29 & 0.40 & 0.16 & 0.30 & 0.22 & 0.37 & 0.06 & -0.09 & 0.02 & -0.01 & 0.03 & 0.11 & 0.23 & 0.24 & -0.13 & 0.00 & 0.21 & -0.02 \\
\hline$\sqrt[4]{\sigma}$ & 0.23 & 0.42 & 0.16 & 0.17 & 0.08 & 0.23 & 0.25 & 0.53 & 0.20 & 0.37 & 0.23 & 0.13 & 0.12 & 0.02 & 0.14 & 0.20 & 0.59 & 0.46 & 0.19 & 0.04 & 0.60 & 0.09 \\
\hline$\mu$ & 0.64 & 0.93 & 0.80 & 0.57 & 0.29 & 0.37 & 0.09 & 0.02 & -0.14 & 0.16 & -0.03 & -0.07 & 0.03 & -0.02 & -0.01 & -0.03 & 0.21 & 0.23 & -0.08 & -0.02 & 0.02 & 0.00 \\
\hline$\sqrt[3]{\sigma}$ & 0.17 & 0.57 & 0.55 & 0.25 & 0.09 & 0.22 & 0.25 & 0.79 & 0.35 & 0.70 & 0.11 & 0.14 & 0.06 & 0.02 & 0.08 & 0.20 & 0.34 & 0.39 & 0.19 & 0.02 & 0.26 & 0.05 \\
\hline$\mu$ & 0.61 & 1.20 & 0.64 & 0.51 & 0.28 & 0.32 & 0.09 & 0.09 & 0.21 & 1.18 & -0.01 & 0.06 & 0.02 & -0.01 & -0.00 & 0.04 & 0.17 & 0.19 & -0.08 & -0.01 & 0.01 & 0.00 \\
\hline$\sqrt[6]{\sigma}$ & 0.21 & 1.75 & 0.59 & 0.31 & 0.10 & 0.18 & 0.23 & 0.90 & 0.73 & 2.20 & 0.12 & 0.13 & 0.06 & 0.03 & 0.09 & 0.21 & 0.28 & 0.35 & 0.17 & 0.03 & 0.25 & 0.06 \\
\hline$\mu$ & 2.40 & 5.34 & 0.82 & 0.67 & 0.33 & 0.30 & 0.02 & 1.92 & 0.42 & 1.85 & 0.05 & -0.04 & 0.01 & -0.01 & 0.01 & 0.07 & 0.23 & 0.18 & -0.04 & 0.01 & 0.71 & 0.01 \\
\hline$\sigma$ & 3.93 & 8.83 & 0.47 & 0.32 & 0.19 & 0.17 & 0.40 & 4.31 & 0.83 & 3.08 & 0.46 & 0.19 & 0.20 & 0.05 & 0.27 & 0.44 & 0.45 & 0.30 & 0.30 & 0.08 & 2.23 & 0.16 \\
\hline$\mu$ & 4.92 & 7.63 & 1.53 & 1.50 & 1.05 & 0.53 & 0.01 & 4.07 & 0.18 & 0.60 & 0.12 & 0.07 & 0.02 & -0.02 & 0.05 & 0.06 & 0.30 & 0.21 & -0.05 & -0.02 & 1.46 & -0.01 \\
\hline$\sqrt[8]{\sigma}$ & 10.36 & 16.57 & 2.49 & 2.33 & 1.44 & 0.21 & 0.49 & 10.51 & 0.73 & 1.32 & 0.59 & 0.19 & 0.27 & 0.06 & 0.35 & 0.65 & 0.81 & 0.36 & 0.33 & 0.11 & 3.94 & 0.21 \\
\hline$\mu$ & 0.47 & 0.52 & 0.39 & 0.36 & 0.28 & 0.39 & 0.20 & 0.42 & -0.25 & 0.44 & 0.01 & -0.12 & 0.00 & -0.01 & 0.01 & -0.13 & 0.44 & 0.38 & -0.16 & -0.01 & 0.01 & -0.01 \\
\hline $9 \sqrt[9]{\sigma}$ & 0.22 & 0.24 & 0.16 & 0.16 & 0.09 & 0.29 & 0.19 & 0.26 & 0.22 & 0.27 & 0.13 & 0.13 & 0.08 & 0.02 & 0.08 & 0.10 & 0.93 & 0.57 & 0.15 & 0.03 & 0.25 & 0.04 \\
\hline$\mu$ & 0.57 & 0.80 & 0.45 & 0.40 & 0.27 & 0.32 & 0.10 & 0.18 & 0.31 & 1.51 & -0.02 & -0.07 & 0.03 & -0.01 & 0.01 & 0.02 & 0.25 & 0.21 & -0.09 & -0.01 & 0.00 & 0.01 \\
\hline$\sigma$ & 0.22 & 0.49 & 0.23 & 0.11 & 0.06 & 0.21 & 0.21 & 0.93 & 0.83 & 2.81 & 0.19 & 0.11 & 0.10 & 0.02 & 0.12 & 0.21 & 0.38 & 0.31 & 0.15 & 0.04 & 0.42 & 0.07 \\
\hline$\mu$ & 2.85 & 12.50 & 0.69 & 0.57 & 0.31 & 0.37 & 0.13 & 3.57 & 1.07 & 4.66 & 0.01 & -0.08 & 0.02 & -0.01 & -0.00 & 0.05 & 0.62 & 0.28 & -0.11 & -0.00 & 0.24 & 0.03 \\
\hline$\sigma$ & 5.36 & 27.71 & 0.62 & 0.44 & 0.16 & 0.23 & 0.24 & 8.64 & 2.61 & 10.72 & 0.43 & 0.12 & 0.22 & 0.03 & 0.26 & 0.46 & 1.18 & 0.36 & 0.19 & 0.06 & 1.25 & 0.18 \\
\hline$\mu$ & 10.68 & 55.89 & 2.94 & 2.19 & 0.92 & 0.49 & 0.10 & 13.60 & 1.11 & 6.92 & 0.11 & -0.10 & 0.03 & -0.01 & 0.04 & 0.04 & 0.46 & 0.35 & -0.12 & 0.01 & 3.02 & -0.01 \\
\hline$\sigma$ & 24.65 & 135.32 & 6.02 & 4.06 & 1.18 & 0.19 & 0.59 & 34.28 & 2.98 & 17.09 & 0.46 & 0.27 & 0.22 & 0.04 & 0.27 & $\begin{array}{l}0.77 \\
\end{array}$ & 1.12 & 0.33 & 0.42 & 0.05 & 7.49 & 0.19 \\
\hline$\mu$ & 0.77 & 2.08 & 0.64 & 0.41 & 0.67 & 0.28 & 0.15 & 0.03 & 0.07 & 0.73 & 0.13 & 0.08 & 0.05 & -0.01 & 0.06 & -0.10 & 0.05 & 0.13 & -0.12 & 0.01 & 0.89 & 0.03 \\
\hline$\sigma$ & 0.37 & 2.84 & 0.35 & 0.12 & 0.81 & 0.12 & 0.27 & 0.99 & 0.76 & 2.18 & 0.39 & 0.13 & 0.17 & 0.03 & 0.21 & 0.32 & 0.27 & 0.19 & 0.20 & 0.05 & 2.31 & 0.13 \\
\hline$\mu$ & 9.85 & 32.73 & 1.86 & 2.28 & 0.67 & 0.36 & 0.04 & 12.19 & 1.26 & 10.46 & 0.01 & -0.06 & 0.01 & -0.00 & -0.01 & 0.09 & 0.30 & 0.22 & -0.06 & -0.01 & 0.21 & 0.02 \\
\hline$\sigma$ & 22.69 & 78.20 & 3.69 & 4.53 & 0.81 & 0.13 & 0.51 & 30.82 & 3.31 & 25.78 & 0.36 & 0.17 & 0.19 & 0.03 & 0.22 & 0.66 & 0.59 & 0.16 & 0.32 & 0.05 & 1.00 & 0.16 \\
\hline$\mu$ & 4.84 & 22.71 & 0.84 & 0.63 & 0.56 & 0.36 & 0.13 & 8.62 & 1.66 & 10.11 & 0.16 & -0.07 & 0.06 & -0.01 & 0.08 & -0.01 & 0.85 & 0.13 & -0.11 & 0.03 & 1.05 & -0.02 \\
\hline$\sigma$ & 10.36 & 53.72 & 0.61 & 0.32 & 0.34 & 0.13 & 0.40 & 21.93 & 3.69 & 20.57 & 0.51 & 0.21 & 0.24 & 0.03 & 0.28 & 0.59 & 2.40 & 0.24 & 0.31 & 0.03 & 2.52 & 0.18 \\
\hline \multirow[b]{2}{*}{ jo } & 0.81 & 1.00 & 0.96 & 0.67 & 0.61 & 0.31 & 0.20 & 0.15 & 0.37 & 2.61 & 0.20 & 0.11 & 0.08 & -0.00 & 0.09 & -0.14 & 0.15 & 0.18 & 0.16 & 0.02 & 2.40 & -0.05 \\
\hline & 0.70 & 0.75 & 0.95 & 0.28 & 0.48 & 0.12 & 0.28 & 1.61 & 1.43 & 6.79 & 0.53 & 0.14 & 0.24 & 0.02 & 0.27 & 0.37 & 1.06 & 0.16 & 0.22 & 0.06 & 5.88 & 0.15 \\
\hline
\end{tabular}

Table 26: Frequency domain parameters SESSION 4 - SESSION 1 difference for EC TRT therapy 
$\underline{\text { Time domain parameters }}$

\begin{tabular}{|c|c|c|c|c|c|c|c|c|c|c|c|c|c|c|c|c|c|c|c|c|c|}
\hline Chann & Mean & MeanAbs & Median & Max & $\min$ & Peak & SSI & IEEG & WL & MMAV1 & \begin{tabular}{|l|} 
MMAV2 \\
\end{tabular} & STD DEV & VAR & ROOT & RMS & CREST & SKEWNESS & KURTOSIS & \begin{tabular}{|l|} 
CLEARANCE \\
\end{tabular} & SHAPE & IMPULSE \\
\hline \multirow{2}{*}{1} & 7.28 & 0.28 & 36.00 & -0.09 & 0.14 & 0.09 & 1.88 & 0.28 & 0.11 & 0.31 & \begin{tabular}{|l|}
0.56 \\
\end{tabular} & 0.24 & 1.69 & 0.29 & 0.27 & 0.08 & \begin{tabular}{|l|}
2.34 \\
\end{tabular} & 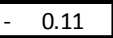 & \begin{tabular}{|l|}
0.04 \\
\end{tabular} & $\begin{array}{ll}-0.02 \\
\end{array}$ & 0.05 \\
\hline & 13.94 & 1.26 & 85.38 & 0.60 & 0.94 & 0.88 & 3.98 & 1.26 & 0.29 & 1.27 & 1.60 & 1.17 & 3.62 & 1.28 & 1.24 & 0.30 & 4.69 & 0.23 & 0.29 & 0.10 & 0.26 \\
\hline \multirow[b]{2}{*}{2} & 1.14 & 0.31 & 0.88 & 0.14 & -0.44 & -0.32 & 0.36 & -0.31 & 0.11 & 0.32 & 2.78 & 0.31 & 0.36 & -0.30 & 0.31 & 0.11 & 2.38 & 0.11 & 0.07 & 0.03 & 0.09 \\
\hline & 1.17 & 0.43 & 1.38 & 0.44 & 0.26 & 0.42 & 0.61 & 0.43 & 0.55 & 0.42 & 6.11 & 0.45 & 0.60 & 0.42 & 0.45 & 0.33 & 4.60 & 0.35 & 0.42 & 0.11 & 0.37 \\
\hline \multirow{2}{*}{3} & 3.35 & 0.69 & 2.18 & 0.39 & 0.70 & 0.29 & 4.70 & 0.69 & 0.04 & 0.71 & 0.84 & 0.64 & 4.57 & 0.71 & 0.64 & 0.18 & 2.00 & 0.22 & 0.19 & 0.03 & 0.20 \\
\hline & 9.14 & 1.95 & 7.96 & 1.41 & 1.33 & 1.40 & 11.76 & 1.95 & 0.38 & 1.95 & 1.91 & 1.86 & 11.44 & 1.98 & 1.90 & 0.09 & 2.38 & 0.24 & 0.17 & 0.06 & 0.13 \\
\hline \multirow{2}{*}{4} & 11.65 & 0.08 & 1.95 & 0.20 & -0.03 & -0.15 & 0.10 & -0.08 & -0.07 & 0.10 & 0.18 & 0.09 & 0.11 & -0.06 & 0.09 & 0.05 & 0.90 & 0.03 & 0.08 & -0.01 & 0.07 \\
\hline & 27.15 & 0.29 & 4.24 & 0.16 & 0.36 & 0.22 & 0.54 & 0.29 & 0.21 & 0.30 & 0.33 & 0.28 & 0.54 & 0.29 & 0.29 & 0.13 & 3.03 & 0.09 & 0.13 & 0.04 & 0.12 \\
\hline \multirow{2}{*}{5} & 9.45 & 0.17 & 1.11 & -0.19 & -0.19 & -0.20 & 0.17 & -0.17 & -0.11 & 0.17 & 0.14 & 0.19 & 0.19 & -0.17 & 0.18 & 0.02 & 0.83 & 0.11 & 0.01 & -0.01 & 0.02 \\
\hline & 23.59 & 0.42 & 2.87 & 0.35 & 0.33 & 0.36 & 0.76 & 0.42 & 0.18 & 0.42 & 0.39 & 0.44 & 0.77 & 0.42 & 0.42 & 0.19 & 1.25 & 0.26 & 0.19 & 0.02 & 0.19 \\
\hline \multirow[b]{2}{*}{6} & 1.00 & 0.14 & 2.33 & -0.04 & -0.14 & -0.09 & 0.24 & -0.14 & -0.08 & 0.16 & 0.13 & 0.19 & 0.27 & -0.12 & 0.16 & 0.11 & 1.12 & 0.03 & 0.07 & 0.03 & 0.09 \\
\hline & 3.18 & 0.22 & 5.51 & 0.34 & 0.44 & 0.31 & 0.47 & 0.22 & 0.15 & 0.21 & 0.26 & 0.29 & 0.50 & 0.22 & 0.26 & 0.34 & 1.82 & 0.23 & 0.41 & 0.10 & 0.38 \\
\hline \multirow{2}{*}{7} & 4.98 & 0.51 & 2.20 & 0.48 & 0.44 & 0.34 & 2.05 & 0.51 & -0.04 & 0.49 & 1.70 & 0.52 & 2.03 & 0.50 & 0.53 & 0.06 & 1.63 & 0.10 & 0.09 & -0.01 & 0.07 \\
\hline & 4.48 & 0.90 & 2.21 & 0.87 & 0.73 & 0.74 & 2.62 & 0.90 & 0.20 & 0.83 & 3.24 & 0.93 & 2.65 & 0.87 & 0.93 & 0.15 & 3.65 & 0.30 & 0.11 & 0.07 & 0.12 \\
\hline \multirow{2}{*}{8} & 38.81 & 2.47 & 75.53 & 1.13 & 1.32 & 1.18 & 32.74 & 2.47 & 0.15 & 2.43 & 2.67 & 1.85 & 24.14 & 2.71 & 2.17 & 0.03 & 1.66 & 0.12 & 0.04 & 0.01 & 0.04 \\
\hline & 91.98 & 6.07 & 183.50 & 3.01 & 3.31 & 2.99 & 79.31 & 6.07 & 0.42 & 5.82 & 5.81 & 4.52 & 58.15 & 6.67 & 5.33 & 0.26 & 40.88 & 0.30 & 0.30 & 0.05 & 0.28 \\
\hline \multirow{2}{*}{9} & 3.92 & 0.04 & 4.29 & -0.39 & -0.04 & -0.10 & 0.80 & 0.04 & -0.18 & 0.07 & 0.27 & 0.23 & 0.28 & 0.06 & 0.02 & 0.10 & 0.81 & 0.08 & 0.13 & 0.01 & 0.12 \\
\hline & 7.30 & 1.05 & 4.68 & 0.22 & 0.71 & 0.53 & 3.34 & 1.05 & 0.25 & 1.17 & 1.71 & 0.39 & 0.72 & 1.12 & 0.96 & 0.37 & 3.58 & 0.22 & 0.44 & 0.05 & 0.41 \\
\hline \multirow{2}{*}{10} & 1.10 & 0.06 & 19.40 & -0.05 & -0.03 & 0.06 & 0.03 & -0.06 & -0.11 & 0.09 & 0.14 & 0.09 & 0.05 & -0.05 & 0.07 & 0.18 & 13.21 & 0.10 & 0.15 & 0.01 & 0.16 \\
\hline & 2.47 & 0.32 & 35.81 & 0.38 & 0.40 & 0.38 & 0.65 & 0.32 & 0.14 & 0.32 & 0.31 & 0.39 & 0.68 & 0.31 & 0.35 & 0.30 & 39.45 & 0.20 & 0.33 & 0.06 & 0.31 \\
\hline \multirow{2}{*}{11} & 36.32 & 0.87 & 0.34 & 0.77 & 0.48 & 0.57 & 6.67 & 0.87 & -0.09 & 0.77 & 1.03 & 0.87 & 6.78 & 0.87 & 0.86 & 0.02 & 9.86 & 0.03 & 0.04 & 0.01 & 0.03 \\
\hline & 83.60 & 2.27 & 8.82 & 1.79 & 1.34 & 1.49 & 16.16 & 2.27 & 0.19 & 1.98 & 2.27 & 2.27 & 16.46 & 2.28 & 2.24 & 0.24 & 24.77 & 0.19 & 0.25 & 0.01 & 0.25 \\
\hline \multirow{2}{*}{12} & 4.80 & 2.44 & 18.96 & 1.68 & 0.62 & 1.50 & 42.86 & 2.44 & -0.06 & 2.49 & 3.42 & 2.27 & 42.78 & 2.55 & 2.30 & -0.11 & 2.18 & 0.13 & 0.21 & -0.07 & 0.17 \\
\hline & 12.16 & 6.56 & 48.13 & 4.80 & 2.47 & 4.41 & 105.76 & 6.56 & 0.37 & 6.71 & 8.86 & 6.30 & 105.59 & 6.79 & 6.29 & 0.16 & 4.06 & 0.15 & 0.24 & 0.09 & 0.20 \\
\hline \multirow{2}{*}{13} & 4.01 & 0.13 & 1.74 & -0.09 & -0.03 & -0.09 & 0.07 & $\begin{array}{l}0.13 \\
\end{array}$ & -0.09 & 0.15 & 0.13 & 0.12 & 0.06 & -0.13 & 0.12 & -0.01 & 1.99 & 0.12 & 0.01 & 0.01 & 0.00 \\
\hline & 8.06 & 0.57 & 6.68 & 0.69 & 0.68 & 0.71 & 1.31 & 0.57 & 0.12 & 0.53 & 0.49 & 0.59 & 1.31 & 0.55 & 0.59 & 0.13 & 4.15 & 0.33 & 0.17 & 0.03 & 0.15 \\
\hline \multirow{2}{*}{14} & 71.20 & 1.48 & 11.83 & 1.29 & 0.35 & 1.11 & 21.48 & 1.48 & -0.07 & 1.35 & 1.73 & 1.50 & 20.15 & 1.46 & 1.56 & 0.03 & 2.86 & 0.00 & 0.10 & 0.03 & 0.07 \\
\hline & 178.20 & 4.23 & 24.68 & 3.91 & 1.51 & 3.42 & 53.06 & 4.23 & 0.16 & 3.91 & 5.06 & 4.23 & 49.83 & 4.18 & 4.37 & 0.38 & 3.48 & 0.13 & 0.51 & 0.05 & 0.45 \\
\hline \multirow{2}{*}{15} & 55.34 & 0.87 & 15.67 & 0.41 & 0.19 & 0.38 & 8.55 & 0.87 & -0.09 & 0.75 & 0.91 & 0.83 & 8.34 & 0.89 & 0.85 & 0.03 & 266.71 & 0.05 & 0.11 & -0.04 & 0.08 \\
\hline & 132.87 & 2.71 & 37.22 & 1.61 & 1.19 & 1.63 & 21.24 & 2.71 & 0.19 & 2.41 & 2.64 & 2.68 & 20.73 & 2.74 & 2.71 & 0.20 & 659.81 & 0.26 & $\begin{array}{l}0.17 \\
\end{array}$ & 0.07 & 0.17 \\
\hline \multirow{2}{*}{16} & 8.37 & 0.01 & 9.01 & 0.01 & -0.10 & -0.06 & 0.60 & 0.01 & -0.03 & 0.03 & 0.15 & 0.01 & 0.59 & 0.00 & 0.01 & 0.07 & 1.91 & 0.08 & 0.09 & 0.01 & 0.08 \\
\hline & 15.37 & 0.84 & 12.06 & 0.66 & 0.71 & 0.69 & 2.55 & 0.84 & 0.15 & 0.75 & 0.51 & 0.84 & 2.54 & 0.84 & 0.84 & 0.31 & 8.19 & 0.07 & 0.34 & 0.02 & 0.33 \\
\hline
\end{tabular}

Table 27: Time domain parameters SESSION 4 - SESSION 1 difference for EC TRT therapy 
EAE therapy (data from 4 patients)

Frequency domain parameters

\begin{tabular}{|c|c|c|c|c|c|c|c|c|c|c|c|c|c|c|c|c|c|c|c|c|c|c|}
\hline \multirow{2}{*}{ Channel } & PSDtotal & PSD- $\delta$ & PSD- $\theta$ & PSD- $\alpha$ & $\begin{array}{l}\text { PSD- } \beta \\
\end{array}$ & PSD- & $\mathrm{P} 1(\mathrm{Xmf})$ & $\mathrm{P} 2$ & P3 & P4 & $\mathrm{P5}(\mathrm{Xfc})$ & P6 & P7(Xrmsf) & P8 & $\mathrm{P9}$ & $\mathrm{P} 10$ & P11 & P12 & $\mathrm{P} 13$ & P14 & MDF & PSDef \\
\hline & 2.33 & 2.50 & 0.62 & 0.57 & 0.61 & 0.53 & 0.33 & 2.67 & 2.00 & 12.84 & 0.38 & -0.22 & 0.14 & -0.04 & 0.20 & 0.19 & 0.09 & 0.41 & -0.28 & 0.04 & 5.42 & -0.07 \\
\hline$\sqrt[1]{\sigma}$ & 2.00 & 1.85 & 0.36 & 0.30 & 0.24 & 0.22 & 0.49 & 5.27 & 2.98 & 15.64 & 0.85 & 0.27 & 0.35 & 0.05 & 0.43 & 0.58 & 0.57 & 0.32 & 0.40 & 0.11 & 10.79 & 0.24 \\
\hline \multirow{2}{*}{\begin{tabular}{l|l}
2 & $\mu$ \\
$\sigma$
\end{tabular}} & 0.88 & 0.97 & 0.76 & 0.59 & 0.53 & 0.42 & 0.46 & 0.51 & 0.37 & 0.50 & 0.61 & -0.27 & 0.24 & -0.05 & 0.33 & 0.38 & 0.04 & 0.35 & -0.38 & 0.06 & 7.83 & -0.15 \\
\hline & 0.18 & 0.26 & 0.18 & 0.30 & 0.28 & 0.02 & 0.36 & 0.69 & 0.35 & 0.39 & 0.85 & 0.22 & 0.35 & 0.05 & 0.43 & 0.45 & 0.48 & 0.21 & 0.31 & 0.10 & 13.96 & 0.22 \\
\hline$\mu$ & 0.71 & 0.61 & 0.54 & 0.44 & 0.41 & 0.49 & 0.10 & 0.16 & 0.23 & 0.29 & 0.18 & 0.10 & 0.07 & -0.03 & 0.11 & 0.18 & 0.25 & 0.09 & -0.10 & 0.04 & 0.73 & 0.07 \\
\hline$\sqrt[3]{\sigma}$ & 0.25 & 0.38 & 0.33 & 0.37 & 0.30 & 0.21 & 0.31 & 0.90 & 0.41 & 0.49 & 0.27 & 0.20 & 0.13 & 0.07 & 0.19 & 0.33 & 0.52 & 0.25 & 0.24 & 0.05 & 0.98 & 0.11 \\
\hline$\mu$ & 0.87 & 1.42 & 0.55 & 0.52 & 0.37 & 0.47 & 0.04 & 0.86 & 0.71 & 4.51 & 0.09 & 0.00 & 0.03 & -0.03 & 0.07 & 0.03 & 0.13 & 0.10 & 0.02 & 0.01 & 0.52 & -0.04 \\
\hline$\sqrt[4]{\sigma}$ & 0.60 & 1.69 & 0.43 & 0.31 & 0.11 & 0.26 & 0.34 & 2.24 & 1.74 & 8.75 & 0.26 & 0.19 & 0.12 & 0.04 & 0.16 & 0.34 & 0.26 & 0.18 & 0.25 & 0.04 & 1.09 & 0.10 \\
\hline$\mu$ & 0.46 & 0.56 & 0.48 & 0.43 & 0.33 & 0.32 & 0.10 & 0.17 & -0.20 & 0.25 & 0.15 & -0.07 & 0.06 & -0.02 & 0.09 & 0.12 & 0.06 & 0.01 & -0.08 & -0.00 & 0.57 & -0.05 \\
\hline$\sqrt[5]{\sigma}$ & 0.34 & 0.28 & 0.34 & 0.26 & 0.19 & 0.09 & 0.29 & 0.55 & 0.35 & 0.55 & 0.33 & 0.15 & 0.16 & 0.02 & 0.19 & 0.30 & 0.31 & 0.12 & 0.22 & 0.04 & 1.18 & 0.11 \\
\hline$\mu$ & 0.90 & 0.95 & 0.62 & 0.51 & 0.32 & 0.33 & 0.14 & 0.41 & 0.04 & 0.25 & 0.25 & 0.12 & 0.07 & -0.04 & 0.14 & 0.13 & 0.02 & 0.18 & -0.13 & 0.00 & 2.91 & -0.07 \\
\hline$\sqrt[6]{\sigma}$ & 0.56 & 0.51 & 0.24 & 0.28 & 0.15 & 0.06 & 0.46 & 1.41 & 0.71 & 1.31 & 0.63 & 0.27 & 0.25 & 0.05 & 0.34 & 0.46 & 0.27 & 0.37 & 0.38 & 0.05 & 6.03 & 0.18 \\
\hline$\mu$ & 1.39 & 2.41 & 0.68 & 0.57 & 0.43 & 0.45 & 0.29 & 0.67 & 0.37 & 2.33 & 0.16 & 0.22 & 0.03 & -0.06 & 0.10 & -0.19 & 0.44 & 0.99 & -0.26 & -0.04 & 2.13 & -0.05 \\
\hline$\sigma$ & 0.87 & 3.05 & 0.31 & 0.35 & 0.35 & 0.34 & 0.51 & 2.15 & 1.45 & 5.49 & 0.59 & 0.34 & 0.27 & 0.07 & 0.31 & 0.51 & 1.03 & 1.77 & 0.42 & 0.11 & 4.76 & 0.18 \\
\hline$\mu$ & 4.70 & 10.91 & 0.69 & 0.50 & 0.61 & 0.63 & 0.31 & 5.13 & 3.38 & 34.83 & 0.56 & 0.14 & 0.26 & -0.04 & 0.33 & -0.29 & 0.19 & 0.00 & -0.24 & 0.08 & 2.89 & -0.13 \\
\hline 8 & 7.52 & 19.97 & 0.30 & 0.35 & 0.25 & 0.31 & 0.40 & 12.15 & 7.66 & 70.64 & 0.72 & 0.26 & 0.34 & 0.06 & 0.44 & 0.52 & 0.62 & 0.33 & 0.31 & 0.11 & 4.05 & 0.19 \\
\hline$\mu$ & 0.70 & 0.86 & 0.82 & 0.83 & 0.43 & 0.36 & 0.20 & 0.32 & 0.40 & 0.58 & 0.35 & -0.13 & 0.14 & -0.04 & 0.20 & -0.24 & 0.24 & 0.02 & -0.16 & -0.00 & 2.22 & -0.10 \\
\hline $9 \sqrt[9]{\sigma}$ & 0.30 & 0.25 & 0.49 & 0.52 & 0.22 & 0.11 & 0.39 & 0.79 & 0.29 & 0.33 & 0.54 & 0.21 & 0.23 & 0.04 & 0.29 & 0.40 & 0.43 & 0.11 & 0.30 & 0.03 & 4.02 & 0.15 \\
\hline$\mu$ & 0.49 & 0.61 & 0.42 & 0.43 & 0.24 & 0.32 & 0.05 & 0.05 & 0.13 & 0.17 & 0.04 & 0.06 & 0.01 & -0.03 & 0.03 & -0.07 & 0.02 & 0.05 & -0.05 & 0.03 & 0.24 & -0.03 \\
\hline$1 0 \longdiv { \sigma }$ & 0.27 & 0.31 & 0.25 & 0.26 & 0.10 & 0.18 & 0.20 & 0.63 & 0.38 & 0.56 & 0.21 & 0.12 & $\begin{array}{ll}0.11 \\
\end{array}$ & 0.03 & 0.12 & 0.23 & 0.29 & $\begin{array}{ll}0.17 \\
\end{array}$ & 0.15 & 0.04 & 0.56 & 0.08 \\
\hline$\mu$ & 5.68 & 18.35 & 1.31 & 0.91 & 1.01 & 0.71 & 0.29 & 6.64 & 2.93 & 39.70 & 0.53 & 0.19 & 0.21 & -0.03 & 0.27 & -0.25 & 0.01 & 0.39 & -0.27 & 0.07 & 7.89 & -0.12 \\
\hline$\sigma$ & 9.51 & 34.81 & 0.78 & 0.11 & 0.48 & 0.25 & 0.73 & 15.10 & 6.31 & 79.39 & 0.86 & 0.42 & 0.33 & 0.06 & 0.42 & 0.68 & 0.43 & 0.55 & 0.56 & 0.09 & 15.24 & 0.21 \\
\hline$\mu$ & 4.92 & 16.62 & 2.27 & 1.49 & 0.87 & 0.47 & 0.02 & 5.98 & 1.44 & 14.60 & 0.29 & 0.02 & 0.13 & -0.02 & 0.16 & 0.05 & 0.07 & 0.07 & -0.04 & 0.05 & 1.74 & 0.07 \\
\hline$\sigma$ & 8.45 & 31.69 & 2.98 & 1.77 & 1.03 & 0.43 & 0.69 & 13.30 & 3.75 & 30.22 & 0.51 & 0.32 & 0.26 & 0.04 & 0.29 & 0.67 & 0.47 & 0.25 & 0.49 & 0.09 & 3.15 & $\begin{array}{ll}0.17 \\
\end{array}$ \\
\hline$\mu$ & 0.52 & 0.58 & 0.57 & 0.47 & 1.08 & 0.29 & 0.14 & 0.06 & 0.01 & 0.46 & 0.27 & -0.09 & 0.11 & -0.03 & 0.15 & -0.15 & 0.13 & 0.04 & -0.12 & 0.03 & 2.48 & -0.08 \\
\hline$\sigma$ & 0.36 & 0.32 & 0.43 & 0.24 & 1.16 & 0.11 & 0.41 & 0.75 & 0.50 & 1.15 & 0.55 & 0.25 & 0.22 & 0.05 & 0.29 & 0.40 & 0.19 & 0.28 & 0.34 & 0.05 & 4.96 & 0.16 \\
\hline$\mu$ & 6.03 & 6.50 & 4.68 & 2.15 & 1.08 & 0.67 & 0.03 & 7.79 & 1.42 & 8.44 & 0.29 & -0.10 & 0.09 & -0.03 & 0.14 & 0.03 & 0.52 & 0.61 & -0.09 & 0.04 & 4.73 & 0.04 \\
\hline$\sigma$ & 10.41 & 10.96 & 7.27 & 3.06 & 1.16 & 0.32 & 0.90 & 16.14 & 2.36 & 15.17 & 0.83 & 0.46 & 0.34 & 0.07 & 0.44 & 0.81 & 0.99 & 1.14 & 0.66 & 0.08 & 9.86 & 0.24 \\
\hline$\mu$ & 3.39 & 4.35 & 0.94 & 0.67 & 0.54 & 0.63 & 0.13 & 3.06 & 0.55 & 1.46 & 0.54 & 0.08 & 0.20 & -0.03 & 0.26 & -0.13 & 0.09 & 0.12 & 0.11 & 0.06 & 10.58 & -0.10 \\
\hline$\sqrt[15]{\sigma}$ & 5.41 & 7.34 & 0.53 & 0.37 & 0.17 & 0.28 & 0.51 & 7.17 & 1.53 & 3.36 & 1.00 & 0.34 & 0.37 & 0.05 & 0.46 & 0.59 & 1.13 & 0.64 & 0.45 & 0.08 & 20.95 & 0.24 \\
\hline \multirow[b]{2}{*}{$\sigma$} & 9.56 & 14.93 & 2.43 & 1.40 & 0.89 & 0.45 & 0.00 & 14.26 & 1.75 & 13.30 & 0.27 & -0.02 & 0.12 & -0.01 & 0.14 & 0.01 & 0.06 & 0.02 & -0.02 & 0.03 & 2.13 & -0.05 \\
\hline & 17.61 & 28.20 & 3.44 & 1.70 & 0.97 & 0.30 & 0.65 & 30.04 & 4.05 & 27.23 & 0.50 & 0.33 & 0.26 & 0.04 & 0.29 & 0.82 & 1.01 & 0.43 & 0.48 & 0.07 & 3.98 & 0.21 \\
\hline
\end{tabular}

Table 28: Frequency domain parameters SESSION 4 - SESSION 1 difference for EC EAE therapy 


\section{$\underline{\text { Time domain parameters }}$}

\begin{tabular}{|c|c|c|c|c|c|c|c|c|c|c|c|c|c|c|c|c|c|c|c|c|c|c|}
\hline Chanr & & Mean & MeanAbs & Median & Max & $\min$ & Peak & SSI & IEEG & WL & MMAV1 & MMAV2 & STD DEV & VAR & ROOT & RMS & CREST & SKEWNESS & KURTOSIS & CLEARANCE & SHAPE & IMPULSE \\
\hline \multirow{2}{*}{\begin{tabular}{l|l}
1 & $\mu$ \\
& $\sigma$
\end{tabular}} & & 2.29 & 0.01 & 1.46 & 0.04 & -0.25 & -0.17 & 1.02 & -0.01 & -0.35 & -0.02 & 0.15 & 0.03 & 1.11 & -0.02 & 0.01 & 0.12 & 0.06 & 0.10 & 0.07 & -0.02 & 0.08 \\
\hline & & 2.47 & 1.11 & 0.54 & 1.13 & 0.88 & 0.91 & 2.65 & 1.11 & 0.27 & 1.10 & 0.96 & 1.18 & 2.82 & 1.08 & 1.15 & 0.51 & 1.41 & 0.19 & 0.39 & 0.06 & 0.43 \\
\hline \multirow[b]{2}{*}{2} & $\mu$ & 0.17 & 0.23 & 0.36 & -0.38 & -0.27 & -0.37 & 0.40 & -0.23 & -0.26 & 0.20 & 0.04 & 0.30 & 0.27 & -0.21 & -0.27 & 0.08 & 0.75 & 0.21 & 0.06 & -0.02 & 0.07 \\
\hline & $\sigma$ & 3.06 & 1.14 & 2.96 & 0.76 & 1.10 & 0.88 & 2.63 & 1.14 & 0.21 & 1.17 & 1.39 & 1.01 & 2.37 & 1.18 & 1.07 & 0.29 & 0.84 & 0.08 & 0.40 & 0.07 & 0.36 \\
\hline \multirow{2}{*}{3} & $\mu$ & 11.14 & 0.18 & 11.00 & 0.09 & 0.24 & 0.11 & 0.03 & -0.18 & -0.02 & 0.21 & 0.33 & 0.17 & -0.04 & -0.17 & -0.17 & 0.14 & 20.50 & 0.04 & 0.11 & -0.01 & 0.13 \\
\hline & $\sigma$ & 19.93 & 0.59 & 19.77 & 0.55 & 0.60 & 0.56 & 0.97 & 0.59 & 0.31 & 0.57 & 0.56 & 0.60 & 0.96 & 0.58 & 0.61 & 0.27 & 40.27 & 0.26 & 0.29 & 0.06 & 0.30 \\
\hline \multirow{2}{*}{4} & $\mu$ & 1.74 & 0.03 & 0.63 & -0.14 & 0.17 & -0.03 & 0.28 & 0.03 & 0.02 & 0.03 & 0.06 & 0.06 & 0.34 & 0.03 & 0.03 & -0.03 & 13.95 & 0.02 & 0.02 & 0.01 & 0.02 \\
\hline & $\sigma$ & 0.48 & 0.56 & 4.54 & 0.53 & 0.37 & 0.44 & 1.39 & 0.56 & 0.18 & 0.58 & 0.65 & 0.53 & 1.40 & 0.56 & 0.54 & 0.13 & 32.11 & 0.24 & 0.20 & 0.05 & 0.18 \\
\hline \multirow{2}{*}{5} & $\mu$ & 0.91 & 0.05 & 1.16 & 0.10 & 0.09 & 0.10 & 0.13 & 0.05 & -0.05 & 0.01 & 0.06 & 0.09 & 0.27 & 0.05 & 0.04 & 0.02 & 0.80 & 0.04 & 0.01 & 0.01 & 0.01 \\
\hline & $\sigma$ & 1.05 & 0.24 & 1.28 & 0.50 & 0.49 & 0.53 & 0.54 & 0.24 & 0.25 & 0.21 & 0.05 & 0.35 & 0.83 & 0.24 & 0.25 & 0.24 & 0.95 & 0.07 & 0.26 & 0.02 & 0.26 \\
\hline \multirow{2}{*}{6} & $\mu$ & 0.28 & 0.21 & 0.28 & 0.16 & 0.12 & -0.08 & 0.96 & 0.21 & -0.11 & 0.24 & 0.22 & 0.18 & 0.95 & 0.22 & 0.18 & -0.15 & 2.53 & 0.20 & 0.21 & 0.04 & 0.19 \\
\hline & $\sigma$ & 2.31 & 0.90 & 1.91 & 0.59 & 0.84 & 0.65 & 1.96 & 0.90 & 0.29 & 0.94 & 0.96 & $\begin{array}{l}0.87 \\
\end{array}$ & 1.91 & 0.91 & 0.87 & 0.15 & 4.29 & 0.16 & 0.10 & 0.05 & 0.11 \\
\hline \multirow{2}{*}{7} & $\mu$ & 14.91 & 0.07 & 4.01 & -0.34 & -0.13 & -0.17 & 0.68 & -0.07 & -0.25 & 0.05 & 0.01 & 0.25 & 0.07 & -0.05 & -0.10 & -0.04 & 1.77 & 0.15 & 0.13 & -0.07 & 0.10 \\
\hline & $\sigma$ & 25.53 & 1.12 & 5.84 & 0.71 & 0.89 & 0.94 & 2.70 & 1.12 & 0.37 & 1.17 & 1.22 & 0.82 & 1.53 & 1.15 & 1.08 & 0.12 & 5.19 & 0.25 & 0.16 & 0.06 & 0.14 \\
\hline \multirow[b]{2}{*}{8} & $\mu$ & 2.17 & 0.25 & 78.23 & -0.26 & -0.26 & -0.27 & 0.18 & -0.25 & -0.20 & 0.25 & 0.32 & 0.24 & 0.20 & -0.25 & -0.25 & 0.17 & 0.47 & 0.09 & 0.15 & -0.01 & 0.15 \\
\hline & $\sigma$ & 6.28 & 0.91 & 154.49 & 0.83 & 0.77 & 0.80 & 1.88 & 0.91 & 0.35 & 0.92 & 0.75 & 0.91 & 1.88 & 0.92 & 0.91 & 0.28 & 2.92 & 0.13 & 0.23 & 0.03 & 0.25 \\
\hline \multirow{2}{*}{9} & $\mu$ & 1.05 & 0.44 & 1.06 & -0.42 & -0.46 & -0.47 & 0.54 & -0.44 & -0.05 & 0.42 & 0.34 & 0.41 & -0.48 & -0.43 & -0.44 & 0.03 & 0.82 & 0.20 & 0.02 & -0.04 & 0.01 \\
\hline & $\sigma$ & 0.20 & 0.46 & 0.33 & 0.45 & 0.35 & 0.40 & 0.49 & 0.46 & 0.29 & 0.48 & 0.55 & 0.49 & 0.56 & 0.46 & 0.46 & 0.16 & 1.80 & 0.22 & 0.14 & 0.04 & 0.15 \\
\hline \multirow{2}{*}{1} & $\mu$ & 15.42 & 0.09 & 2.32 & 0.12 & -0.01 & -0.03 & 0.42 & 0.09 & -0.08 & 0.12 & 0.20 & 0.08 & 0.37 & 0.09 & 0.09 & -0.04 & 0.53 & 0.00 & 0.05 & 0.01 & 0.05 \\
\hline & $\sigma$ & 29.82 & 0.54 & 5.99 & 0.60 & 0.33 & 0.42 & 1.36 & 0.54 & 0.29 & 0.64 & 0.84 & 0.54 & 1.25 & 0.53 & 0.56 & 0.27 & 0.40 & 0.14 & 0.26 & 0.02 & 0.26 \\
\hline \multirow{2}{*}{1} & $\mu$ & 0.34 & 0.20 & $\begin{array}{l}0.17 \\
\end{array}$ & -0.31 & -0.24 & -0.25 & 0.26 & -0.20 & -0.26 & 0.20 & 0.29 & 0.19 & 0.25 & -0.20 & -0.19 & 0.03 & 0.82 & 0.01 & 0.02 & -0.01 & 0.03 \\
\hline & $\sigma$ & 2.54 & 0.88 & 1.24 & 0.76 & 0.79 & 0.80 & 1.78 & 0.88 & 0.40 & 0.86 & 0.72 & 0.88 & 1.72 & 0.88 & 0.89 & 0.47 & 1.68 & 0.25 & 0.44 & 0.03 & 0.46 \\
\hline \multirow{2}{*}{1} & $\mu$ & 16.48 & 0.16 & 4.47 & -0.24 & -0.14 & -0.21 & 0.25 & -0.16 & -0.02 & 0.17 & 0.08 & 0.20 & 0.16 & -0.16 & -0.17 & 0.14 & 4.59 & 0.09 & 0.19 & 0.01 & 0.16 \\
\hline & $\sigma$ & 26.34 & 0.86 & 4.03 & 0.73 & 0.91 & 0.77 & 1.77 & 0.86 & 0.32 & 0.87 & 0.96 & 0.83 & 1.65 & 0.87 & 0.86 & 0.40 & 6.71 & 0.16 & 0.53 & 0.04 & 0.46 \\
\hline & $\mu$ & 2.53 & 0. & 0.2 & -0.05 & 0.11 & -0.01 & 1.22 & 0.21 & -0.03 & 0.18 & 0.0 & $\begin{array}{ll}0.17 \\
\end{array}$ & 1.20 & 0.22 & 0.1 & 0.02 & 0.23 & 0.11 & 0.04 & 0.03 & 0.01 \\
\hline & $\sigma$ & 4.59 & 1.07 & 3.00 & 0.89 & 0.83 & 0.82 & 3.03 & 1.07 & 0.25 & 1.05 & 0.84 & 1.05 & 3.01 & 1.08 & 1.05 & 0.36 & 2.94 & 0.12 & 0.29 & 0.02 & 0.33 \\
\hline \multirow[t]{2}{*}{1} & $\mu$ & 2.35 & 0.23 & 0.24 & -0.18 & -0.19 & -0.19 & 0.08 & -0.23 & -0.17 & 0.24 & 0.34 & 0.24 & -0.13 & -0.24 & -0.23 & 0.19 & 84.19 & 0.04 & 0.29 & 0.03 & 0.25 \\
\hline & $\sigma$ & 7.45 & 0.64 & 2.68 & 0.77 & 0.75 & 0.76 & 1.12 & 0.64 & 0.40 & 0.62 & 0.47 & 0.63 & 1.03 & 0.64 & 0.66 & 0.42 & 169.44 & 0.13 & 0.60 & 0.06 & 0.52 \\
\hline \multirow[t]{2}{*}{ 1 } & $\mu$ & 6.28 & 0.10 & 6.15 & 0.02 & -0.25 & -0.08 & 0.26 & -0.10 & 0.03 & 0.12 & 0.22 & 0.12 & 0.14 & -0.10 & -0.08 & 0.14 & 3.77 & 0.04 & 0.09 & 0.01 & 0.12 \\
\hline & $\sigma$ & 12.05 & 0.73 & 11.49 & 0.83 & 0.57 & 0.71 & 1.36 & 0.73 & 0.40 & 0.68 & 0.55 & 0.69 & 1.16 & 0.72 & 0.75 & 0.25 & 2.81 & 0.11 & $\begin{array}{l}0.11 \\
\end{array}$ & 0.06 & 0.17 \\
\hline \multirow{2}{*}{$\frac{\mu}{\sigma}$} & ㅅ & 3.18 & 0.02 & 1.74 & 0.08 & -0.23 & -0.03 & 0.94 & 0.02 & -0.01 & 0.04 & 0.39 & 0.03 & 0.86 & 0.00 & 0.05 & -0.04 & 1.58 & 0.19 & 0.13 & -0.05 & 0.10 \\
\hline & $\sigma$ & 4.60 & 0.97 & 2.12 & 0.99 & 0.71 & 1.03 & 2.74 & 0.97 & 0.19 & 0.87 & 0.56 & 1.03 & 2.55 & 0.92 & 1.06 & 0.18 & 7.98 & 0.20 & 0.17 & 0.13 & 0.13 \\
\hline
\end{tabular}

Table 29: Time domain parameters SESSION 4 - SESSION 1 difference for EC EAE therapy 
- RS therapy (data from 1 patient)

Frequency domain parameters

\begin{tabular}{|c|c|c|c|c|c|c|c|c|c|c|c|c|c|c|c|c|c|c|c|c|c|c|}
\hline Channel & PSDtotal & PSD- $\delta$ & PSD $-\theta$ & PSD- $\alpha$ & PSD- $\beta$ & PSD- $\gamma$ & P1(Xmf) & P2 & P3 & P4 & $\mathrm{P5}(\mathrm{Xfc})$ & P6 & P7(Xrmsf) & P8 & P9 & P10 & P11 & P12 & P13 & P14 & MDF & PSDef \\
\hline 1 & 1.29 & 0.34 & 0.54 & 1.58 & 1.62 & 1.78 & 0.53 & 0.70 & -0.61 & 0.86 & \begin{tabular}{|l|}
0.05 \\
\end{tabular} & 0.17 & 0.02 & -0.02 & 0.04 & 0.11 & 0.35 & -0.34 & 0.36 & -0.05 & 0.09 & -0.03 \\
\hline 2 & 0.53 & 0.14 & 0.60 & 1.42 & 0.80 & 0.23 & 0.08 & 0.52 & 0.07 & 0.05 & -0.12 & -0.00 & 0.09 & -0.03 & -0.06 & 0.13 & 0.58 & 0.03 & 0.06 & -0.04 & 0.18 & 0.03 \\
\hline 3 & 0.49 & 0.54 & 0.27 & 1.01 & 0.45 & 0.28 & 0.05 & 0.24 & -0.53 & 0.75 & 0.02 & -0.02 & 0.01 & -0.02 & 0.02 & -0.04 & 0.02 & 0.03 & 0.03 & -0.04 & 0.02 & 0.02 \\
\hline 4 & 0.58 & 0.53 & 0.55 & 1.48 & 0.41 & 0.58 & 0.15 & 0.04 & -0.39 & 0.69 & -0.20 & -0.13 & 0.15 & -0.02 & -0.13 & 0.09 & 1.15 & 0.57 & -0.12 & -0.06 & 0.38 & 0.07 \\
\hline 5 & 0.83 & 0.64 & 0.90 & 1.74 & 0.81 & 0.55 & 0.01 & 0.24 & -0.59 & 0.87 & -0.26 & -0.15 & 0.21 & -0.04 & -0.18 & 0.15 & 0.98 & 0.83 & -0.06 & -0.15 & 0.33 & 0.07 \\
\hline 6 & 1.05 & 1.86 & 0.80 & 2.25 & 0.58 & 0.68 & 0.07 & 1.08 & 0.17 & 0.19 & -0.33 & -0.18 & 0.25 & -0.03 & -0.24 & 0.24 & 1.47 & 1.17 & -0.11 & -0.15 & 0.46 & 0.12 \\
\hline 7 & 0.58 & 0.68 & 0.22 & 0.55 & 0.31 & 0.63 & 0.24 & 0.35 & -0.39 & 0.60 & -0.15 & -0.19 & 0.11 & -0.01 & -0.10 & -0.05 & 1.08 & 0.73 & -0.20 & -0.06 & 0.31 & 0.04 \\
\hline 8 & 0.91 & 0.91 & 0.42 & 1.24 & 0.62 & 0.95 & 0.62 & 0.82 & -0.65 & 0.88 & -0.34 & -0.42 & 0.24 & -0.01 & -0.23 & -0.12 & 9.46 & 3.08 & -0.52 & -0.06 & 0.58 & 0.15 \\
\hline 9 & 0.74 & 0.34 & 0.28 & 1.93 & 0.85 & 0.67 & 0.14 & 0.43 & -0.30 & 0.62 & -0.32 & -0.26 & 0.27 & -0.10 & -0.19 & 0.10 & 1.35 & 1.08 & -0.15 & -0.20 & 0.43 & 0.08 \\
\hline 10 & 0.76 & 0.40 & 0.33 & 1.28 & 0.49 & 0.54 & 0.10 & -0.41 & -0.71 & 0.92 & -0.20 & -0.19 & 0.18 & -0.04 & -0.14 & 0.02 & 0.74 & 0.81 & -0.12 & -0.15 & 0.22 & 0.03 \\
\hline 11 & 0.74 & 0.85 & 0.35 & 0.32 & 0.45 & 0.87 & 0.53 & -0.68 & -0.25 & 0.63 & -0.22 & -0.37 & 0.17 & -0.03 & -0.15 & -0.18 & 4.25 & 2.01 & -0.44 & -0.08 & 0.40 & 0.06 \\
\hline 12 & 0.75 & 0.75 & 0.44 & 0.64 & 0.43 & 0.88 & 0.51 & 0.66 & -0.53 & 0.84 & -0.25 & -0.37 & 0.19 & -0.03 & -0.16 & -0.17 & 2.70 & 2.18 & -0.43 & -0.10 & 0.46 & 0.08 \\
\hline 13 & 1.07 & 1.31 & 0.40 & 2.33 & 0.37 & 0.62 & 0.06 & 1.12 & 0.63 & 1.96 & -0.30 & -0.19 & 0.24 & -0.04 & -0.21 & 0.17 & 1.06 & 1.06 & -0.11 & -0.16 & 0.37 & 0.09 \\
\hline 14 & 0.47 & 0.44 & 0.47 & 0.47 & 0.37 & 0.62 & 0.29 & 0.25 & 0.13 & 0.25 & -0.17 & -0.24 & 0.14 & -0.03 & -0.11 & -0.08 & 1.11 & 1.10 & -0.26 & -0.10 & 0.26 & 0.04 \\
\hline 15 & 0.86 & 0.91 & 0.75 & 0.58 & 0.73 & 0.95 & 0.68 & 0.84 & -0.33 & 0.68 & -0.24 & -0.47 & 0.17 & 0.00 & -0.17 & -0.30 & 4.27 & 3.63 & -0.58 & -0.06 & 0.47 & 0.09 \\
\hline 16 & 0.70 & 0.65 & 0.15 & 0.71 & 0.28 & 0.83 & 0.44 & 0.40 & -0.24 & 0.63 & 0.29 & 0.35 & 0.23 & 0.05 & -0.19 & -0.08 & 4.74 & 2.13 & 0.37 & 0.13 & 0.48 & 0.08 \\
\hline
\end{tabular}

Table 30: Frequency domain parameters SESSION 4 - SESSION 1 difference for EC RS therapy

Time domain parameters

\begin{tabular}{|c|c|c|c|c|c|c|c|c|c|c|c|c|c|c|c|c|c|c|c|c|c|}
\hline Channel & Mean & MeanAbs & Median & Max & $\min$ & Peak & SSI & IEEG & WL & MMAV1 & MMAV2 & STD DEV & VAR & ROOT & RMS & CREST & SKEWNESS & KURTOSIS & CLEARANCE & SHAPE & IMPULSE \\
\hline 1 & 1.15 & 1.04 & 1.27 & 0.57 & 0.97 & 0.57 & 3.12 & 1.04 & 1.27 & 1.03 & 1.05 & 1.05 & 3.20 & 1.04 & 1.03 & -0.23 & 1.26 & 0.04 & 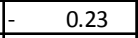 & \begin{tabular}{|l|}
-0.00 \\
\end{tabular} & 0.23 \\
\hline 2 & 7.30 & 1.36 & 5.58 & 1.12 & 1.17 & 1.12 & 4.64 & 1.36 & 0.57 & 1.36 & 1.34 & 1.14 & 3.57 & 1.34 & 1.37 & -0.11 & 6.21 & 0.01 & 0.10 & 0.01 & 0.10 \\
\hline 3 & 1.69 & 0.57 & 12.07 & 0.26 & 1.04 & 0.26 & 1.18 & 0.57 & 0.94 & 0.67 & 1.77 & 0.45 & 1.11 & 0.62 & 0.48 & -0.15 & 0.98 & 0.28 & 0.22 & -0.06 & 0.20 \\
\hline 4 & 1.97 & 0.53 & 1.56 & 0.23 & 0.42 & 0.42 & 1.34 & 0.53 & 0.12 & 0.54 & 0.63 & 0.54 & 1.37 & 0.52 & 0.53 & -0.07 & 2.96 & 0.05 & 0.07 & 0.00 & 0.07 \\
\hline 5 & 1.21 & 0 & 0.76 & 0.03 & 0.39 & 0.10 & 0.37 & 0.17 & 0.18 & 0.19 & 0.29 & 0.17 & 0.37 & 0.16 & 0.17 & -0.06 & 0.98 & 0.01 & 0.05 & 0.00 & 0.06 \\
\hline 6 & 0.27 & 0.76 & 0.49 & 0.99 & 0.65 & 0.98 & 2.09 & 0.76 & 0.03 & 0.77 & 0.89 & 0.76 & 2.11 & 0.77 & 0.76 & 0.13 & 1.02 & 0.00 & 0.12 & -0.00 & 0.12 \\
\hline 7 & 17.51 & 0.36 & 3.86 & 0.40 & 0.17 & 0.20 & 0.81 & 0.36 & 0.13 & 0.37 & 0.54 & 0.35 & 0.81 & 0.37 & 0.35 & -0.10 & 0.93 & 0.06 & 0.12 & -0.01 & 0.11 \\
\hline 8 & 9.77 & 0.21 & 1.73 & -0.14 & -0.27 & -0.25 & 0.38 & -0.21 & -0.58 & 0.25 & 0.26 & 0.26 & 0.46 & -0.20 & -0.21 & -0.05 & 0.91 & 0.01 & 0.06 & -0.01 & 0.05 \\
\hline 9 & 0.99 & 0.34 & 1.05 & -0.24 & -0.46 & -0.32 & 0.59 & -0.34 & -0.02 & 0.35 & 0.36 & 0.35 & 0.58 & -0.33 & -0.36 & 0.07 & 1.26 & 0.20 & 0.02 & -0.03 & 0.04 \\
\hline 10 & 5.8 & & & 1.90 & 1.48 & 1.90 & 6.20 & 1.68 & 0.42 & 53 & 1.42 & 1.68 & 6.19 & 1.68 & 1.68 & 0.08 & 4.96 & 0.03 & 0.08 & 0.00 & 0.08 \\
\hline 11 & 3.02 & 0.07 & 8.46 & -0.03 & -0.16 & -0.10 & 0.13 & -0.07 & -0.27 & -0.09 & 0.08 & 0.07 & 0.13 & -0.07 & -0.07 & 0.04 & 0.29 & 0.04 & 0.04 & -0.00 & 0.04 \\
\hline 12 & 1.28 & 0.22 & 1.85 & 0.20 & 0.30 & 0.20 & 0.50 & 0.22 & -0.31 & 0.22 & 0.28 & 0.23 & 0.51 & 0.23 & 0.22 & 0.02 & 3.88 & 0.04 & 0.02 & 0.00 & 0.02 \\
\hline 13 & 5.71 & 0.98 & 3.57 & 0.67 & 0.79 & 0.67 & 3.01 & 0.98 & 0.20 & 1.02 & 1.06 & 1.00 & 2.99 & 0.95 & 1.00 & -0.17 & 2.32 & 0.05 & 0.15 & 0.01 & 0.16 \\
\hline 14 & 1.58 & & & 0.11 & -0.13 & -0.01 & 0.41 & 0.18 & 0.02 & 0.17 & 0.13 & 0.19 & 0.41 & 0.17 & 0.19 & -0.17 & 3.04 & 0.05 & 0.16 & 0.01 & 0.16 \\
\hline 15 & 2.52 & 0.38 & -72.98 & -0.37 & -0.44 & -0.38 & 0.62 & -0.38 & -0.56 & -0.39 & 0.41 & 0.38 & 0.62 & -0.39 & -0.38 & 0.00 & 0.95 & 0.00 & 0.01 & 0.00 & 0.00 \\
\hline 16 & 0.21 & 1.00 & 2.65 & 1.05 & 0.53 & 0.67 & 2.92 & 1.00 & -0.21 & 1.10 & 1.76 & 0.98 & 2.93 & 1.00 & 0.98 & -0.16 & 1.52 & 0.09 & 0.16 & -0.01 & 0.16 \\
\hline
\end{tabular}

Table 31: Time domain parameters SESSION 4 - SESSION 1 difference for EC RS therapy 
ANNEX Z: SESSION 4 - SESSION 1 DIFFERENCE OF TIME AND TREQUENCY DOMAIN PARAMETERS FROM EACH ACOUSTIC THERAPY FOR THERAPY: SELECTION FOR FRONTAL AND TEMPORAL LOBE CHANNELS

\begin{tabular}{|c|c|c|c|c|c|c|c|c|c|c|c|c|c|c|c|}
\hline & & \multicolumn{4}{|c|}{ Time domain parameters selection } & \multicolumn{10}{|c|}{ Frequency domain parameters selection } \\
\hline Binaura & & Mean & Peak & RMS & KURTOSIS & PSDtotal & PSD- $\delta$ & PSD- $\theta$ & PSD- $\alpha$ & PSD- $\beta$ & PSD- $\gamma$ & P1(Xmf) & P4 & $\mathrm{P5}(\mathrm{Xfc})$ & P13 \\
\hline \multirow{2}{*}{1} & $\mu$ & $-\quad 3.21$ & 0.24 & 0.38 & 0.04 & 2.54 & 6.78 & 1.06 & 0.21 & 0.33 & 0.66 & -0.09 & 3.55 & $-\quad 0.31$ & $-\quad 0.08$ \\
\hline & $\sigma$ & 5.81 & 1.10 & 1.29 & 0.28 & - & 0.00 & - & 0.00 & - & 0.00 & 0.00 & 0.00 & - & 0.00 \\
\hline \multirow{2}{*}{2} & $\mu$ & 6.33 & 1.05 & 1.43 & 0.34 & 0.78 & 1.80 & 0.29 & 0.16 & 0.20 & 0.44 & 0.07 & 0.29 & 0.17 & 0.06 \\
\hline & $\sigma$ & 30.09 & 1.97 & 2.62 & 0.64 & 0.00 & 0.00 & 0.00 & - & 0.00 & - & - & - & 0.00 & - \\
\hline \multirow{2}{*}{3} & $\mu$ & 1.59 & 0.57 & 0.57 & 0.02 & 0.53 & 0.36 & 0.24 & 0.26 & 0.25 & 0.49 & 0.20 & 0.82 & 0.12 & 0.16 \\
\hline & $\sigma$ & 15.18 & 1.85 & 1.95 & 0.24 & - & - & - & 0.00 & - & - & 0.00 & - & 0.00 & - \\
\hline \multirow{2}{*}{7} & $\mu$ & 48.16 & 2.16 & 2.56 & 0.16 & 0.65 & 0.68 & 0.43 & 0.52 & 0.35 & 0.68 & 0.39 & 0.71 & 0.10 & 0.32 \\
\hline & $\sigma$ & 142.20 & 4.38 & 5.64 & 0.26 & 0.00 & - & 0.00 & - & - & 0.00 & 0.00 & 0.00 & - & - \\
\hline EAE & & Mean & Peak & RMS & KURTOSIS & PSDtotal & PSD- $\delta$ & PSD- $\theta$ & $\overline{P S D}-\alpha$ & PSD- $\beta$ & PSD- $\gamma$ & $\mathrm{P} 1$ (Xmf) & P4 & $P 5(X f c)$ & P13 \\
\hline \multirow{2}{*}{1} & $\mu$ & 12.19 & \begin{tabular}{|l|}
5.67 \\
\end{tabular} & \begin{tabular}{|l|}
6.08 \\
\end{tabular} & \begin{tabular}{|l|}
0.10 \\
\end{tabular} & \begin{tabular}{|l|}
40.39 \\
\end{tabular} & 45.54 & 5.78 & 1.51 & 0.63 & \begin{tabular}{|l|}
0.60 \\
\end{tabular} & \begin{tabular}{|l|}
0.55 \\
\end{tabular} & 0.46 & $-\quad 0.00$ & \begin{tabular}{|l}
0.29 \\
\end{tabular} \\
\hline & $\sigma$ & 48.53 & 17.54 & 19.40 & 0.21 & 131.95 & 147.10 & 17.31 & 3.17 & 0.76 & 0.53 & 1.62 & 1.12 & 0.22 & 0.80 \\
\hline \multirow{2}{*}{2} & $\mu$ & 2.32 & 0.49 & 0.45 & 0.09 & 1.94 & 2.09 & 0.80 & 0.52 & 0.46 & 0.43 & 0.02 & 0.60 & 0.18 & 0.03 \\
\hline & $\sigma$ & 8.50 & 1.83 & 1.79 & 0.33 & 3.62 & 3.16 & 1.38 & 0.51 & 0.36 & 0.21 & 0.38 & 1.93 & 0.43 & 0.27 \\
\hline \multirow{2}{*}{3} & $\mu$ & 15.93 & 0.29 & 0.42 & 0.11 & 1.31 & 1.50 & 0.79 & 0.76 & 0.75 & 0.55 & 0.04 & 0.86 & 0.08 & 0.01 \\
\hline & $\sigma$ & 50.24 & 0.75 & 1.02 & 0.21 & 1.13 & 1.57 & 0.81 & 0.71 & 0.68 & 0.28 & 0.42 & 2.74 & 0.56 & 0.30 \\
\hline \multirow{2}{*}{7} & $\mu$ & 0.73 & 0.25 & 0.33 & 0.12 & 0.61 & 0.60 & 0.49 & 0.42 & 0.32 & 0.41 & 0.09 & 0.45 & 0.10 & 0.08 \\
\hline & $\sigma$ & 0.83 & 0.24 & 0.21 & 0.19 & 0.24 & 0.25 & 0.50 & 0.18 & 0.17 & 0.34 & 0.24 & 0.48 & 0.19 & 0.20 \\
\hline TRT & & Mean & Peak & RMS & KURTOSIS & PSDtotal & PSD- $\delta$ & PSD- $\theta$ & PSD- $\alpha$ & PSD- $\beta$ & PSD- $\gamma$ & P1(Xmf) & P4 & $\mathrm{P5}(\mathrm{Xfc})$ & P13 \\
\hline \multirow{2}{*}{1} & $\mu$ & 225.25 & 1.58 & 1.63 & 0.25 & 16.04 & 78.53 & 4.18 & 2.77 & 1.03 & 1.05 & 0.41 & 13.64 & 0.40 & 0.21 \\
\hline & $\sigma$ & 387.45 & 2.62 & 2.28 & 0.17 & 19.46 & 128.01 & 6.06 & 4.33 & 0.96 & 0.49 & 0.95 & 12.06 & 0.08 & 0.61 \\
\hline \multirow{2}{*}{2} & $\mu$ & - $\quad 31.32$ & 0.66 & 0.61 & 0.02 & 10.42 & 6.88 & 3.23 & 2.41 & 1.66 & 0.37 & 0.38 & 3.37 & 0.20 & 0.20 \\
\hline & $\sigma$ & 49.58 & 0.94 & 0.79 & 0.16 & 17.16 & 10.09 & 5.17 & 3.80 & 2.56 & 0.15 & 0.77 & 6.01 & 0.18 & 0.44 \\
\hline \multirow{2}{*}{3} & $\mu$ & 3.24 & 0.60 & 0.48 & 0.40 & 37.32 & 59.87 & 4.27 & 3.48 & 1.83 & 0.45 & 0.71 & 1.70 & 0.26 & 0.38 \\
\hline & $\sigma$ & 13.76 & 0.73 & 0.34 & 0.85 & 63.63 & 102.45 & 5.73 & 5.37 & 2.49 & 0.10 & 1.16 & 2.46 & 0.26 & 0.63 \\
\hline \multirow{2}{*}{ 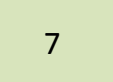 } & $\mu$ & 0.53 & 0.23 & 0.09 & 0.06 & 3.41 & 6.16 & 1.39 & 1.16 & 0.45 & 0.55 & 0.08 & 3.53 & 0.15 & 0.03 \\
\hline & $\sigma$ & 2.18 & 0.51 & 0.39 & 0.11 & 4.74 & 7.90 & 1.53 & 1.17 & 0.23 & 0.19 & 0.41 & 6.04 & 0.18 & 0.30 \\
\hline
\end{tabular}

Table 32: Difference between session 4 and session 1: time and frequency parameters selection for therapy condition analysis 
Acoustic therapy condition: Mean behaviour per therapy in Channels C1, C2, C3 \& C7

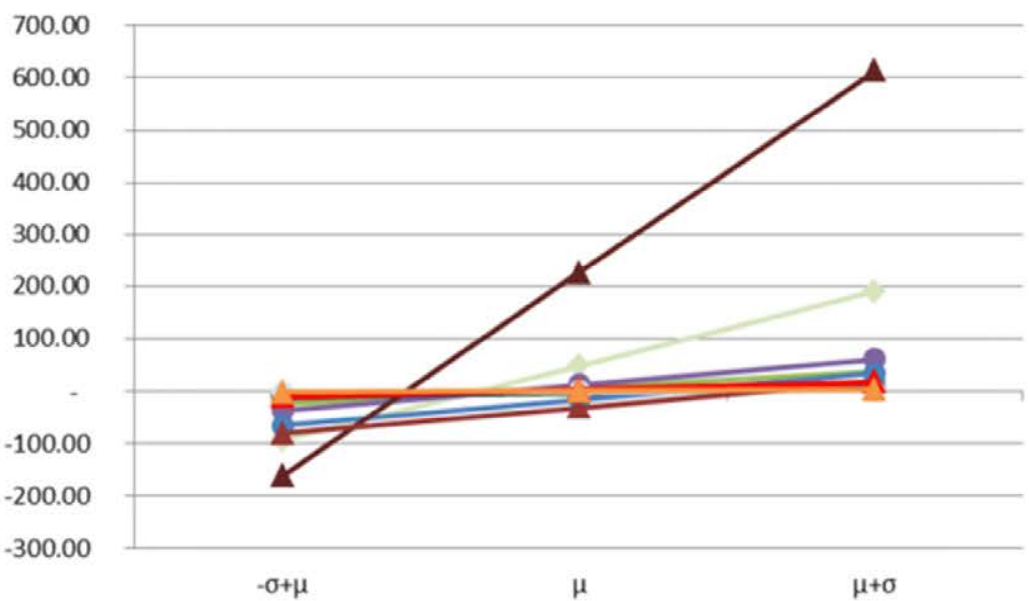

(a)
Acoustic therapy condition: MNF behaviour per therapy in Channels C1, C2, C3 \& C7

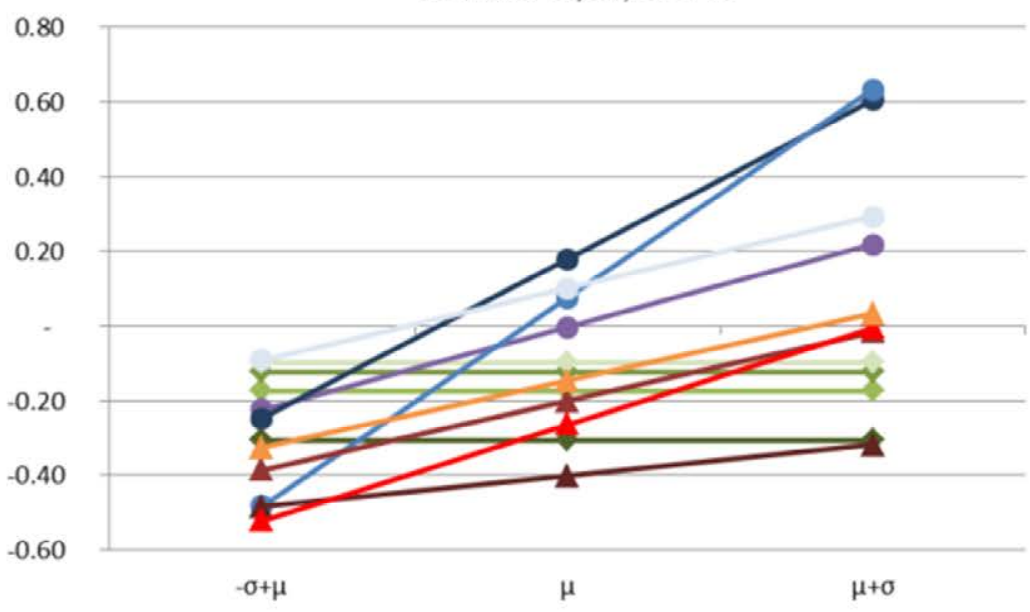

(b)

$$
\begin{aligned}
& \leadsto \text { Binaural C1 } \leadsto \text { Binaural C2 } \leadsto \text { Binaural C3 }- \text { Binaural C7 } \\
& \longrightarrow \text { EAEC1 }- \text { EAE C2 } \longrightarrow \text { EAEC3 } \\
& \text { —TRT C1 }
\end{aligned}
$$

Figure 29: Acoustic therapy condition analysis: Frontal and temporal lobe behavior per therapy shown by the difference between sessions 4 and 1 of the Mean (a) and MNF (b) 


\section{ANNEX AA: SESSION 4 - SESSION 1 DIFFERENCE OF TIME AND TREQUENCY DOMAIN PARAMETERS FROM EACH ACOUSTIC THERAPY FOR THERAPY}

In the following tables are found the Average $(\mu)$ and Standard Deviation $(\sigma)$ of the time and frequency domain parameters difference between session 4 and session 1 (relativized by Session 1) for Acoustic therapy condition of all patients per acoustic therapy: Binaural $396 \mathrm{~Hz}, \mathrm{TRT}$ and EAE.

- Binaural $396 \mathrm{~Hz}$ therapy (data from 7 patients)

Frequency domain parameters

\begin{tabular}{|c|c|c|c|c|c|c|c|c|c|c|c|c|c|c|c|c|c|c|c|c|c|c|c|}
\hline Channel & & PSDtotal & PSD- $\delta$ & PSD $-\theta$ & PSD- $\alpha$ & PSD- $\beta$ & PSD-y & P1(Xmf) & P2 & P3 & P4 & $\mathrm{P} 5(\mathrm{Xfc})$ & P6 & P7(Xrmsf) & P8 & P9 & $\mathrm{P} 10$ & P11 & P12 & $\mathrm{P} 13$ & P14 & MDF & PSDef \\
\hline \multirow{2}{*}{1} & $\underline{\mu}$ & 2.54 & 6.78 & 1.06 & 0.21 & 0.33 & 0.66 & -0.09 & 2.78 & 1.36 & 3.55 & \begin{tabular}{|l|}
-0.31 \\
\end{tabular} & -0.06 & \begin{tabular}{|l|l|} 
& 0.19 \\
\end{tabular} & 0.01 & -0.19 & 0.35 & 2.11 & 0.59 & -0.08 & -0.01 & 0.61 & 0.17 \\
\hline & $\sigma$ & - & 0.00 & - & 0.00 & - & 0.00 & 0.00 & 0.00 & 0.00 & 0.00 & - & 0.00 & 0.00 & - & 0.00 & 0.00 & 0.00 & - & 0.00 & 0.00 & - & 0.00 \\
\hline \multirow{2}{*}{2} & $\underline{\mu}$ & 0.78 & 1.80 & 0.29 & 0.16 & 0.20 & 0.44 & 0.07 & 0.61 & 0.15 & 0.29 & -0.17 & -0.05 & 0.10 & 0.01 & -0.11 & 0.15 & 0.71 & 0.34 & -0.06 & -0.01 & 0.39 & 0.09 \\
\hline & $\sigma$ & 0.00 & 0.00 & 0.00 & - & 0.00 & - & - & 0.00 & - & - & 0.00 & 0.00 & - & - & - & - & 0.00 & - & - & - & 0.00 & 0.00 \\
\hline \multirow{2}{*}{3} & $\underline{\mu}$ & 0.53 & 0.36 & 0.24 & 0.26 & 0.25 & 0.49 & 0.20 & 0.00 & 0.42 & 0.82 & -0.12 & -0.12 & 0.08 & -0.02 & -0.07 & 0.01 & 1.05 & 0.35 & -0.16 & -0.01 & 0.22 & 0.05 \\
\hline & $\sigma$ & & & & 0.00 & & & 0.00 & & 0.00 & & 0.00 & 0.00 & 0.00 & & & & 0.00 & 0.00 & & 0.00 & 0.00 & 0.00 \\
\hline \multirow{2}{*}{4} & $\underline{\mu}$ & 1.11 & 3.18 & 0.22 & 0.40 & 0.53 & 0.82 & 0.39 & 0.40 & 1.83 & 4.17 & -0.25 & -0.20 & 0.15 & 0.01 & -0.16 & 0.06 & 2.85 & 1.06 & -0.31 & 0.02 & 0.46 & 0.13 \\
\hline & $\sigma$ & & & 0.00 & 0.00 & 0.00 & 0.00 & 0.00 & - & 0.00 & & 0.00 & 0.00 & & 0.00 & & & & & - & & 0.00 & 0.00 \\
\hline \multirow{2}{*}{5} & $\mu$ & 0.41 & 0.45 & 0.26 & 0.25 & 0.19 & 0.47 & 0.14 & 0.31 & 0.72 & 2.15 & -0.16 & -0.10 & 0.11 & -0.01 & -0.10 & 0.07 & 0.73 & 0.45 & -0.12 & -0.03 & 0.30 & 0.06 \\
\hline & $\sigma$ & 0.00 & 0.00 & - & 0.00 & 0.00 & 0.00 & 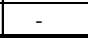 & - & 0.00 & 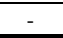 & - & - & 0.00 & - & 0.00 & - & - & - & 0.00 & - & - & 0.00 \\
\hline \multirow{2}{*}{6} & $\mu$ & 0.62 & 1.02 & 0.31 & 0.17 & 0.24 & 0.61 & 0.18 & 0.52 & 0.94 & 3.03 & -0.23 & -0.14 & 0.15 & -0.01 & -0.14 & 0.12 & 1.23 & 0.71 & -0.16 & -0.05 & 0.42 & 0.10 \\
\hline & $\sigma$ & - & 0.00 & 0.00 & - & - & - & - & - & - & 0.00 & 0.00 & - & - & 0.00 & - & 0.00 & - & 0.00 & - & 0.00 & 0.00 & 0.00 \\
\hline \multirow{2}{*}{7} & $\mu$ & 0.65 & 0.68 & 0.43 & 0.52 & 0.35 & 0.68 & 0.39 & 0.67 & -0.45 & 0.71 & -0.10 & -0.26 & 0.08 & -0.02 & -0.06 & -0.18 & 1.77 & 0.91 & -0.32 & -0.05 & 0.20 & 0.02 \\
\hline & $\bar{\sigma}$ & 0.00 & - & 0.00 & - & - & 0.00 & 0.00 & - & - & 0.00 & - & - & - & - & 0.00 & - & 0.00 & 0.00 & - & - & - & 0.00 \\
\hline \multirow{2}{*}{8} & $\underline{\mu}$ & 0.68 & 3.36 & 0.22 & 0.40 & 0.58 & 0.29 & 0.11 & 0.85 & 2.21 & 8.68 & -0.05 & 0.04 & 0.04 & -0.02 & -0.02 & 0.10 & 1.94 & -0.08 & 0.07 & -0.01 & 0.06 & 0.01 \\
\hline & $\sigma$ & 0.00 & 0.00 & 0.00 & 0.00 & - & - & 0.00 & - & - & - & - & 0.00 & 0.00 & - & 0.00 & 0.00 & - & - & - & - & - & 0.00 \\
\hline \multirow{2}{*}{9} & $\mu$ & 0.63 & 1.13 & 0.57 & 0.31 & 0.30 & 0.60 & 0.21 & 0.25 & 0.49 & 0.93 & -0.21 & -0.13 & 0.14 & -0.03 & -0.12 & 0.10 & 2.07 & 0.50 & -0.16 & -0.02 & 0.40 & 0.09 \\
\hline & $\sigma$ & & & 0.00 & & 0.00 & 0.00 & 0.00 & & & & 0.00 & & - & 0.00 & & \begin{tabular}{|l|l}
0.00 \\
\end{tabular} & & 0.00 & - & 0.00 & 0.00 & - \\
\hline \multirow[b]{2}{*}{10} & $\underline{\mu}$ & 0.60 & 0.70 & 0.29 & 0.37 & 0.29 & 0.79 & 0.42 & 0.53 & -0.12 & 0.40 & -0.19 & -0.28 & 0.15 & -0.03 & -0.12 & -0.11 & 3.02 & 1.24 & -0.34 & -0.06 & 0.38 & 0.06 \\
\hline & $\sigma$ & & 0.00 & 0.00 & & - & 0.00 & 0.00 & - & 0.00 & 0.00 & - & - & - & - & 0.00 & - & 0.00 & 0.00 & 0.00 & 0.00 & 0.00 & 0.00 \\
\hline \multirow{2}{*}{11} & $\mu$ & 0.26 & 0.35 & 0.25 & 0.18 & 0.13 & 0.20 & 0.00 & 0.21 & -0.37 & 0.59 & 0.04 & -0.03 & 0.01 & -0.01 & 0.02 & -0.06 & 0.14 & 0.03 & -0.01 & -0.03 & 0.06 & -0.02 \\
\hline & $\sigma$ & - & - & - & - & - & - & 0.00 & 0.00 & - & 0.00 & - & - & 0.00 & - & 0.00 & 0.00 & - & - & - & 0.00 & - & 0.00 \\
\hline \multirow{2}{*}{12} & $\sqrt{\mu}$ & 0.38 & 0.68 & 0.27 & 0.23 & 0.45 & 0.73 & 0.36 & 0.33 & 0.21 & 0.46 & -0.16 & -0.22 & 0.11 & -0.01 & -0.10 & -0.07 & 1.58 & 0.91 & -0.29 & -0.03 & 0.37 & 0.06 \\
\hline & $\sigma$ & - & - & - & 0.00 & 0.00 & - & - & - & 0.00 & 0.00 & 0.00 & 0.00 & 0.00 & - & 0.00 & 0.00 & 0.00 & - & - & \begin{tabular}{|l}
0.00 \\
\end{tabular} & - & - \\
\hline \multirow{2}{*}{13} & $\underline{\mu}$ & 0.28 & 0.24 & 0.57 & 0.21 & 0.26 & 0.53 & 0.15 & 0.05 & 0.02 & 0.04 & -0.17 & -0.12 & 0.12 & -0.01 & -0.11 & 0.06 & 0.83 & 0.57 & -0.13 & -0.05 & 0.34 & 0.07 \\
\hline & $\sigma$ & & 0.00 & & & & & & 0.00 & 0.00 & & 0.00 & 0.00 & 0.00 & 0.00 & & & & & - & 0.00 & & \\
\hline \multirow{2}{*}{14} & $\underline{\mu}$ & 0.51 & 0.56 & 0.45 & 0.58 & 0.26 & 0.24 & 0.21 & 0.64 & -0.54 & 0.81 & 0.08 & -0.12 & 0.04 & -0.00 & 0.04 & -0.18 & 0.05 & 0.21 & -0.16 & -0.01 & 0.20 & -0.04 \\
\hline & $\sigma$ & & 0.00 & 0.00 & 0.00 & & 0.00 & & & 0.00 & 0.00 & & 0.00 & & 0.00 & & 0.00 & 0.00 & 0.00 & 0.00 & 0.00 & & \\
\hline \multirow{2}{*}{15} & $\underline{\mu}$ & 0.66 & 1.47 & 0.22 & 0.20 & 0.21 & 0.41 & 0.12 & 0.58 & 1.12 & 2.83 & -0.14 & -0.07 & 0.09 & -0.01 & -0.08 & 0.08 & 0.76 & 0.29 & -0.09 & -0.01 & 0.30 & 0.06 \\
\hline & $\sigma$ & 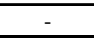 & 0.00 & 0.00 & 0.00 & 0.00 & - & 0.00 & 0.00 & - & - & - & - & 0.00 & - & 0.00 & - & - & - & - & - & - & 0.00 \\
\hline \multirow[b]{2}{*}{16} & $\underline{\mu}$ & 0.46 & 0.26 & 0.26 & 0.29 & 0.24 & 0.30 & 0.12 & 0.30 & 1.50 & 4.12 & -0.06 & -0.06 & 0.04 & -0.00 & -0.03 & -0.00 & 0.33 & 0.19 & -0.09 & 0.00 & 0.12 & 0.02 \\
\hline & o & 0.00 & 0.00 & - & 0.00 & - & 0.00 & 0.00 & 0.00 & 0.00 & - & 0.00 & 0.00 & - & - & - & - & 0.00 & 0.00 & - & - & 0.00 & 0.00 \\
\hline
\end{tabular}

Table 33: Frequency domain parameters SESSION 4 - SESSION 1 difference for Acoustic Therapy - Binaural 396Hz 
$\underline{\text { Time domain parameters }}$

\begin{tabular}{|c|c|c|c|c|c|c|c|c|c|c|c|c|c|c|c|c|c|c|c|c|c|}
\hline Channel & Mean & MeanAbs & Median & Max & $\min$ & Peak & SSI & IEEG & WL & MMAV1 & \begin{tabular}{|l} 
MMAV2 \\
\end{tabular} & STD DEV & VAR & ROOT & RMS & CREST & SKEWNESS & KURTOSIS & \begin{tabular}{|l|} 
CLEARANCE \\
\end{tabular} & \begin{tabular}{|l|l|} 
SHAPE \\
\end{tabular} & \begin{tabular}{|l|} 
IMPULSE \\
\end{tabular} \\
\hline \multirow{2}{*}{1} & 3.21 & $\begin{array}{lll}0.41 \\
\end{array}$ & 1.27 & 0.49 & 0.15 & 0.24 & 2.33 & 0.41 & 0.04 & $\begin{array}{l}0.43 \\
\end{array}$ & \begin{tabular}{|l|}
0.50 \\
\end{tabular} & 0.33 & 2.10 & 0.44 & 0.38 & -0.03 & 4.40 & 0.04 & 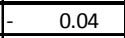 & \begin{tabular}{|l|l|}
-0.01 \\
\end{tabular} & \begin{tabular}{|l|}
-0.04 \\
\end{tabular} \\
\hline & 5.81 & 1.36 & 8.55 & 1.83 & 0.72 & 1.10 & 5.84 & 1.36 & 0.50 & 1.36 & 1.37 & 1.24 & 5.46 & 1.41 & 1.29 & 0.28 & 7.33 & 0.28 & 0.32 & 0.04 & 0.31 \\
\hline \multirow{2}{*}{2} & 6.33 & 1.30 & 7.77 & 1.08 & 0.86 & 1.05 & 10.78 & 1.30 & 0.10 & 1.32 & 1.33 & 1.34 & 9.40 & 1.22 & 1.43 & 0.05 & 6.81 & 0.34 & 0.18 & 0.06 & 0.13 \\
\hline & 30.09 & 2.45 & 25.58 & 1.86 & 1.90 & 1.97 & 19.73 & 2.45 & 0.44 & 2.55 & 2.85 & 2.40 & 16.57 & 2.34 & 2.62 & 0.45 & 22.25 & 0.64 & 0.69 & 0.09 & 0.60 \\
\hline \multirow{2}{*}{3} & 1.59 & 0.56 & 274.92 & 0.55 & 0.56 & 0.57 & 4.72 & 0.56 & 0.09 & 0.58 & 0.94 & 0.56 & 4.65 & 0.57 & 0.57 & 0.05 & 71.47 & 0.02 & 0.06 & 0.00 & 0.06 \\
\hline & 15.18 & 1.91 & 744.02 & 1.86 & 1.82 & 1.85 & 12.90 & 1.91 & 0.48 & 1.93 & 2.40 & 1.93 & 12.75 & 1.92 & 1.95 & 0.11 & 189.02 & 0.24 & 0.17 & 0.04 & 0.14 \\
\hline \multirow{2}{*}{4} & 5.24 & 0.87 & 18.86 & 0.42 & 1.36 & 1.33 & 17.71 & 0.87 & 0.04 & 0.97 & 1.21 & 1.23 & 17.38 & 0.65 & 1.26 & 0.07 & 0.39 & 0.17 & 0.14 & 0.04 & 0.11 \\
\hline & 14.54 & 2.95 & 29.71 & 1.88 & 4.20 & 4.14 & 47.59 & 2.95 & 0.27 & 3.23 & 3.92 & 3.95 & 46.81 & 2.36 & 3.99 & 0.19 & 13.03 & 0.37 & 0.29 & 0.13 & 0.20 \\
\hline \multirow{2}{*}{5} & 204.61 & 5.19 & $-1,261.59$ & 3.53 & 2.90 & 3.53 & 92.06 & 5.19 & 0.32 & 5.28 & 5.66 & 3.72 & 63.09 & 5.55 & 4.72 & 0.12 & 8.70 & 0.04 & 0.16 & 0.04 & 0.15 \\
\hline & 574.01 & 9.13 & $3,296.73$ & 7.34 & 5.53 & 7.13 & 159.96 & 9.13 & 0.98 & 9.25 & 9.74 & 6.98 & 123.86 & 9.73 & 8.39 & 0.18 & 14.37 & 0.17 & 0.21 & 0.04 & 0.20 \\
\hline \multirow{2}{*}{6} & 50.15 & 3.38 & 45.06 & 0.60 & 2.28 & 1.97 & 74.37 & 3.38 & 0.02 & 3.65 & 4.35 & 1.80 & 27.06 & 3.53 & 3.13 & 0.04 & 0.47 & 0.02 & 0.07 & -0.02 & 0.05 \\
\hline & 134.92 & 8.91 & 120.88 & 1.64 & 6.08 & 5.23 & 196.44 & 8.91 & 0.23 & 9.62 & 11.52 & 4.85 & 71.46 & 9.28 & 8.25 & 0.18 & 1.70 & 0.22 & 0.20 & 0.04 & 0.19 \\
\hline \multirow{2}{*}{7} & 48.16 & 2.50 & 40.73 & 1.89 & 1.92 & 2.16 & 38.97 & 2.50 & 0.12 & 2.75 & 3.73 & 2.21 & 30.10 & 2.51 & 2.56 & 0.04 & 0.03 & 0.16 & 0.07 & 0.02 & 0.06 \\
\hline & 142.20 & 5.48 & 113.85 & 3.41 & 4.47 & 4.38 & 99.91 & 5.48 & 0.41 & 6.14 & 8.85 & 4.93 & 77.26 & 5.50 & 5.64 & 0.19 & 2.73 & 0.26 & 0.28 & 0.06 & 0.25 \\
\hline \multirow{2}{*}{8} & 46.32 & 2.27 & 85.66 & 1.17 & 1.01 & 1.17 & 28.90 & 2.27 & -0.17 & 2.56 & 3.41 & 1.63 & 23.25 & 2.49 & 2.04 & 0.11 & 0.69 & 0.08 & 0.12 & -0.01 & 0.12 \\
\hline & 133.43 & 5.47 & 258.07 & 2.95 & 3.17 & 2.94 & 74.48 & 5.47 & 0.29 & 6.18 & 8.18 & 4.49 & 61.64 & 5.99 & 4.91 & 0.16 & 3.20 & 0.14 & 0.23 & 0.07 & 0.20 \\
\hline \multirow[b]{2}{*}{9} & 12.13 & 0.63 & 5.07 & 0.34 & 0.75 & 0.72 & 5.80 & 0.63 & -0.08 & 0.64 & 0.71 & 0.61 & 5.66 & 0.63 & 0.63 & 0.19 & 5.98 & 0.05 & 0.24 & 0.02 & 0.22 \\
\hline & 27.10 & 2.20 & 31.19 & 1.31 & 2.44 & 2.17 & 15.70 & 2.20 & 0.22 & 2.21 & 2.37 & 2.17 & 15.41 & 2.22 & 2.19 & 0.40 & 16.37 & 0.14 & 0.52 & 0.04 & 0.47 \\
\hline \multirow{2}{*}{10} & 27.85 & 4.18 & 15.07 & 4.10 & 3.50 & 4.29 & 105.12 & 4.18 & 0.32 & 3.90 & 3.14 & 4.09 & 93.98 & 4.18 & 4.31 & -0.03 & 65.91 & 0.30 & 0.00 & 0.02 & 0.01 \\
\hline & 63.66 & 9.30 & 122.49 & 9.73 & 7.64 & 9.69 & 269.77 & 9.30 & 1.00 & 8.66 & 7.30 & 8.98 & 240.50 & 9.38 & 9.53 & 0.13 & 143.40 & 0.46 & 0.13 & 0.02 & 0.13 \\
\hline \multirow{2}{*}{11} & 35.89 & 1.29 & 10.82 & 0.81 & 1.24 & 1.21 & 23.74 & 1.29 & 0.06 & 1.56 & 2.22 & 1.57 & 23.68 & 1.07 & 1.57 & 0.05 & 14.97 & 0.03 & 0.00 & 0.02 & 0.03 \\
\hline & 85.23 & 3.83 & 14.66 & 2.67 & 3.76 & 3.78 & 63.36 & 3.83 & 0.35 & 4.55 & 6.06 & 4.59 & 63.23 & 3.23 & 4.60 & 0.19 & 51.45 & 0.20 & 0.23 & 0.08 & 0.20 \\
\hline \multirow{2}{*}{12} & 165.47 & 6.90 & 161.77 & 3.40 & 4.23 & 4.18 & 329.34 & 6.90 & 0.07 & 7.55 & 9.48 & 6.03 & 285.80 & 7.34 & 6.50 & -0.03 & 0.49 & 0.02 & 0.04 & -0.01 & 0.03 \\
\hline & 439.80 & 18.96 & 430.23 & 9.83 & 11.82 & 11.84 & 872.11 & 18.96 & 0.42 & 20.68 & 25.71 & 16.64 & 756.92 & 20.13 & 17.88 & 0.20 & 1.04 & 0.14 & 0.22 & 0.03 & 0.21 \\
\hline \multirow[b]{2}{*}{13} & 27.17 & 0.89 & 32.81 & 0.73 & 0.80 & 0.93 & 5.33 & 0.89 & -0.06 & 0.91 & 0.97 & 0.58 & 3.30 & 0.96 & 0.79 & 0.13 & 4.93 & 0.10 & 0.11 & -0.01 & 0.11 \\
\hline & 52.37 & 2.14 & 59.66 & 1.25 & 2.18 & 2.00 & 13.17 & 2.14 & 0.12 & 2.21 & 2.46 & 1.45 & 8.20 & 2.33 & 1.91 & 0.29 & 5.51 & 0.19 & 0.33 & 0.04 & 0.31 \\
\hline \multirow{2}{*}{14} & -111.94 & 8.01 & 133.19 & 3.33 & 2.58 & 3.05 & 365.08 & 8.01 & -0.01 & 8.03 & 8.61 & 5.69 & 238.27 & 8.81 & 7.08 & -0.03 & 4.21 & 0.04 & 0.04 & -0.02 & 0.03 \\
\hline & 287.01 & 21.20 & 350.21 & 8.87 & 6.72 & 7.93 & 965.59 & 21.20 & 0.41 & 21.29 & 22.95 & 15.06 & 630.12 & 23.31 & 18.73 & 0.26 & 7.89 & 0.06 & 0.29 & 0.04 & 0.28 \\
\hline \multirow{2}{*}{15} & -100.00 & 7.01 & 55.16 & 4.56 & 5.45 & 5.22 & 333.09 & 7.01 & 0.23 & 6.75 & 6.31 & 6.49 & 295.28 & 7.12 & 6.89 & -0.00 & 1.50 & 0.01 & 0.00 & 0.00 & 0.00 \\
\hline & 260.17 & 18.12 & 143.10 & 11.79 & 13.79 & 13.24 & 880.17 & 18.12 & 0.34 & 17.41 & 16.23 & 16.74 & 780.12 & 18.42 & 17.81 & 0.13 & 11.26 & 0.05 & 0.14 & 0.01 & 0.14 \\
\hline \multirow{2}{*}{16} & 8.39 & 1.66 & 16.17 & 1.19 & 0.76 & 1.18 & 19.69 & 1.66 & 0.15 & 1.74 & 1.76 & 1.53 & 17.48 & 1.73 & 1.63 & -0.08 & 4.92 & 0.03 & 0.08 & 0.00 & 0.08 \\
\hline & 24.36 & 4.12 & 32.95 & 2.98 & 1.88 & 2.98 & 51.27 & 4.12 & 0.43 & 4.29 & 4.26 & 3.75 & 45.44 & 4.31 & 4.01 & 0.15 & 11.69 & 0.15 & 0.19 & 0.03 & 0.18 \\
\hline
\end{tabular}

Table 34: Time domain parameters SESSION 4 - SESSION 1 difference for Acoustic Therapy - Binaural 396Hz 
- $\quad$ TRT therapy (data from 3 patients)

Frequency domain parameters

\begin{tabular}{|c|c|c|c|c|c|c|c|c|c|c|c|c|c|c|c|c|c|c|c|c|c|}
\hline Channel & Mean & MeanAbs & Median & Max & $\min$ & Peak & SSI & IEEG & WL & MMAV1 & MMAV2 & STD DEV & VAR & ROOT & RMS & CREST & SKEWNESS & KURTOSIS & CLEARANCE & SHAPE & \begin{tabular}{|l|} 
IMPULSE \\
\end{tabular} \\
\hline \multirow{2}{*}{1} & 225.25 & 1.59 & 95.21 & 1.86 & 1.56 & 1.58 & 9.38 & 1.59 & 0.41 & 1.61 & 1.40 & $\begin{array}{ll}1.57 \\
\end{array}$ & 8.86 & 1.58 & 1.63 & -0.07 & -11.50 & 0.25 & -0.06 & \begin{tabular}{|l|}
0.01 \\
\end{tabular} & \begin{tabular}{|l|}
-0.06 \\
\end{tabular} \\
\hline & 387.45 & 2.23 & 185.93 & 3.18 & 2.51 & 2.62 & 14.50 & 2.23 & 1.65 & 2.19 & 1.67 & 2.21 & 13.67 & 2.20 & 2.28 & 0.19 & 7.42 & 0.17 & 0.20 & 0.01 & 0.20 \\
\hline \multirow{2}{*}{2} & -31.32 & 0.63 & 12.24 & 1.01 & 0.27 & 0.66 & 2.02 & 0.63 & -0.03 & 0.70 & 1.05 & 0.57 & 1.82 & 0.64 & 0.61 & -0.01 & 0.88 & 0.02 & 0.02 & 0.01 & 0.02 \\
\hline & 49.58 & 0.79 & 25.37 & 1.08 & 0.68 & 0.94 & 2.47 & 0.79 & 0.08 & 0.82 & 1.02 & 0.73 & 2.18 & 0.80 & 0.79 & 0.11 & 1.26 & 0.16 & 0.11 & 0.00 & 0.12 \\
\hline \multirow{2}{*}{3} & 3.24 & 0.48 & 48.15 & 0.30 & 0.71 & 0.60 & 1.27 & 0.48 & 0.04 & 0.51 & 0.94 & 0.43 & 1.09 & 0.50 & 0.48 & 0.07 & 0.95 & 0.40 & 0.13 & 0.01 & 0.11 \\
\hline & 13.76 & 0.42 & 95.40 & 0.41 & 0.67 & 0.73 & 0.95 & 0.42 & 0.27 & 0.53 & 1.64 & 0.30 & 0.82 & 0.50 & 0.34 & 0.37 & 8.88 & 0.85 & 0.54 & 0.11 & 0.49 \\
\hline \multirow{2}{*}{4} & 4.71 & 0.99 & 2.34 & 0.90 & 0.98 & 0.82 & 5.21 & 0.99 & 0.14 & 1.03 & 0.82 & 1.08 & 5.14 & 0.91 & 1.10 & 0.09 & 0.10 & 0.31 & 0.03 & 0.04 & 0.05 \\
\hline & 12.80 & 1.47 & 23.37 & 1.14 & 1.78 & 1.30 & 8.43 & 1.47 & 0.40 & 1.47 & 0.98 & 1.64 & 8.38 & 1.35 & 1.64 & 0.26 & 8.63 & 0.18 & 0.26 & 0.04 & 0.26 \\
\hline \multirow{2}{*}{5} & 3.18 & 0.23 & 13.98 & 0.08 & 0.11 & 0.07 & 0.56 & 0.23 & 0.07 & 0.23 & 0.22 & 0.19 & 0.45 & 0.24 & 0.23 & 0.11 & 80.68 & 0.06 & 0.11 & -0.00 & 0.11 \\
\hline & 5.34 & 0.31 & 18.08 & 0.26 & 0.02 & 0.12 & 0.75 & 0.31 & 0.21 & 0.33 & 0.35 & 0.23 & 0.58 & 0.33 & 0.29 & 0.13 & 143.46 & 0.05 & 0.15 & 0.02 & 0.14 \\
\hline \multirow[b]{2}{*}{6} & 0.94 & 0.22 & 11.45 & 0.01 & 0.35 & 0.10 & 0.53 & 0.22 & 0.08 & 0.24 & 0.25 & 0.18 & 0.40 & 0.22 & 0.23 & 0.11 & 6.67 & 0.12 & 0.10 & 0.01 & 0.10 \\
\hline & 7.09 & 0.14 & 9.28 & 0.15 & 0.38 & 0.14 & 0.35 & 0.14 & 0.28 & 0.13 & 0.23 & 0.15 & 0.36 & 0.14 & 0.14 & 0.02 & 6.35 & 0.09 & 0.02 & 0.01 & 0.02 \\
\hline \multirow{2}{*}{7} & 0.53 & 0.09 & 2.81 & 0.00 & 0.47 & 0.23 & 0.30 & 0.09 & 0.07 & 0.10 & 0.08 & 0.09 & 0.30 & 0.10 & 0.09 & 0.10 & 0.38 & 0.06 & 0.10 & -0.00 & 0.10 \\
\hline & 2.18 & 0.39 & 5.41 & 0.36 & 0.70 & 0.51 & 0.79 & 0.39 & 0.41 & 0.39 & 0.36 & 0.40 & 0.80 & 0.39 & 0.39 & 0.08 & 2.68 & 0.11 & 0.09 & 0.01 & 0.09 \\
\hline \multirow{2}{*}{8} & 149.12 & 4.89 & 324.63 & 3.50 & 1.10 & 3.45 & 90.27 & 4.89 & 0.13 & 5.86 & 8.21 & 4.28 & 64.52 & 4.83 & 5.12 & 0.09 & 0.71 & 0.09 & 0.09 & 0.01 & 0.08 \\
\hline & 249.23 & 8.57 & 593.67 & 5.98 & 1.88 & 6.04 & 156.54 & 8.57 & 0.18 & 10.24 & 14.30 & 7.52 & 111.96 & 8.45 & 8.98 & 0.19 & 3.37 & 0.12 & 0.16 & 0.03 & 0.17 \\
\hline \multirow{2}{*}{9} & -6.39 & 1.03 & 9.23 & 0.27 & 1.31 & 0.88 & 6.92 & 1.03 & 0.16 & 0.86 & 0.07 & 0.52 & 2.67 & 1.04 & 1.06 & 0.11 & 7.33 & 0.15 & 0.12 & -0.00 & 0.11 \\
\hline & 18.47 & 2.26 & 8.89 & 0.27 & 2.85 & 2.18 & 12.81 & 2.26 & 0.24 & 1.95 & 0.57 & 1.42 & 5.45 & 2.28 & 2.35 & 0.05 & 15.98 & 0.47 & 0.07 & 0.03 & 0.07 \\
\hline \multirow{2}{*}{10} & 27.73 & 0.11 & 27.38 & 0.09 & 0.12 & 0.00 & 0.35 & 0.11 & 0.21 & 0.09 & 0.06 & 0.08 & 0.29 & 0.11 & 0.10 & 0.07 & 17.97 & 0.02 & 0.07 & -0.00 & 0.07 \\
\hline & 51.57 & 0.44 & 50.49 & 0.37 & 0.45 & 0.37 & 0.87 & 0.44 & 0.35 & 0.43 & 0.42 & 0.43 & 0.83 & 0.44 & 0.44 & 0.16 & 26.91 & 0.09 & 0.18 & 0.02 & 0.17 \\
\hline \multirow{2}{*}{11} & 1.30 & 0.15 & 1.07 & 0.19 & 0.19 & 0.21 & 0.30 & 0.15 & 0.02 & 0.14 & 0.19 & 0.16 & 0.30 & 0.14 & 0.16 & 0.06 & 0.58 & 0.07 & 0.09 & -0.02 & 0.08 \\
\hline & 1.13 & 0.04 & 1.00 & 0.13 & 0.07 & 0.05 & 0.07 & 0.04 & 0.51 & 0.03 & 0.06 & 0.04 & 0.07 & 0.04 & 0.04 & 0.03 & 0.91 & 0.06 & 0.05 & 0.01 & 0.04 \\
\hline \multirow{2}{*}{12} & 36.22 & 1.44 & 13.57 & 0.78 & 1.21 & 1.09 & 7.59 & 1.44 & 0.02 & 1.32 & $\begin{array}{l}1.17 \\
\end{array}$ & 1.29 & 6.33 & 1.44 & 1.44 & -0.04 & 1.97 & 0.07 & 0.03 & & 0.04 \\
\hline & 67.84 & 2.01 & 14.54 & 0.90 & 1.64 & 1.30 & 11.12 & 2.01 & 0.44 & 1.74 & 1.43 & 1.77 & 9.13 & 2.00 & 1.99 & 0.20 & 9.81 & 0.24 & 0.21 & 0.01 & 0.20 \\
\hline \multirow{2}{*}{13} & 16.50 & 1.41 & 16.69 & 1.83 & 0.83 & 1.45 & 8.32 & 1.41 & 0.02 & 1.53 & 1.94 & 1.45 & 7.98 & 1.35 & 1.48 & 0.00 & 0.28 & 0.09 & 0.04 & 0.00 & 0.03 \\
\hline & 31.06 & 2.08 & 15.86 & 2.74 & 0.94 & 2.08 & 13.53 & 2.08 & 0.31 & 2.26 & 2.91 & 2.12 & 12.93 & 2.01 & 2.18 & 0.06 & 3.58 & 0.02 & 0.06 & 0.01 & 0.06 \\
\hline \multirow[b]{2}{*}{14} & 3.16 & 0.14 & 2.80 & 0.32 & 0.19 & 0.25 & 0.27 & 0.14 & 0.01 & 0.12 & 0.04 & 0.16 & 0.28 & 0.13 & 0.15 & $\frac{0.06}{-0.12}$ & 1.99 & 0.11 & 0.14 & 0.02 & 0.13 \\
\hline & 3.27 & 0.14 & 2.64 & 0.10 & 0.27 & 0.11 & 0.22 & 0.14 & 0.36 & 0.16 & 0.34 & 0.13 & 0.20 & 0.14 & 0.13 & 0.03 & 0.63 & 0.07 & 0.02 & 0.01 & 0.02 \\
\hline \multirow{2}{*}{15} & 5.27 & 0.81 & 2.74 & 0.54 & 0.44 & 0.42 & 3.60 & 0.81 & 0.00 & 0.68 & 0.46 & 0.77 & 3.46 & 0.82 & 0.78 & -0.14 & 0.07 & 0.09 & 0.15 & -0.02 & 0.15 \\
\hline & 7.43 & 1.50 & 15.88 & 0.84 & 1.09 & 0.92 & 6.09 & 1.50 & 0.41 & 1.25 & 0.90 & 1.42 & 5.84 & 1.50 & 1.46 & 0.18 & 2.16 & 0.16 & 0.19 & 0.02 & 0.19 \\
\hline \multirow[b]{2}{*}{16} & -183.74 & 1.57 & 47.66 & 0.90 & 0.98 & 0.86 & 11.35 & 1.57 & 0.01 & 1.77 & 2.17 & 1.19 & 7.34 & 1.56 & 1.56 & -0.06 & 2.04 & 0.01 & 0.06 & -0.01 & 0.06 \\
\hline & 312.78 & 2.97 & 80.48 & 1.57 & 1.96 & 1.60 & 20.10 & 2.97 & 0.23 & 3.31 & 4.04 & 2.30 & 13.15 & 2.95 & 2.95 & 0.28 & 3.53 & 0.11 & 0.28 & 0.00 & 0.28 \\
\hline
\end{tabular}

Table 35: Frequency domain parameters SESSION 4 - SESSION 1 difference for Acoustic Therapy - TRT 
$\underline{\text { Time domain parameters }}$

\begin{tabular}{|c|c|c|c|c|c|c|c|c|c|c|c|c|c|c|c|c|c|c|c|c|c|c|}
\hline Channel & PSDtotal & PSD- $\delta$ & PSD- $\theta$ & PSD- $\alpha$ & PSD- $\beta$ & PSD- $\gamma$ & $\mathrm{P} 1$ (Xmf) & P2 & P3 & P4 & $\mathrm{P5}(\mathrm{Xfc})$ & P6 & $\mid \mathrm{P7}$ (Xrmsf) & P8 & P9 & P10 & P11 & P12 & P13 & P14 & MDF & PSDef \\
\hline \multirow{2}{*}{1} & 16.04 & 78.53 & 4.18 & 2.77 & 1.03 & 1.05 & 0.41 & 28.51 & 2.95 & 13.64 & -0.40 & 0.09 & \begin{tabular}{|l|}
-0.25 \\
\end{tabular} & 0.04 & -0.28 & 0.87 & 1.45 & 0.78 & 0.21 & -0.06 & 0.65 & 0.26 \\
\hline & 19.46 & 128.01 & 6.06 & 4.33 & 0.96 & 0.49 & 0.95 & 42.86 & 1.74 & 12.06 & 0.08 & 0.41 & 0.05 & 0.02 & 0.07 & 0.85 & 0.66 & 1.11 & 0.61 & 0.07 & 0.07 & 0.08 \\
\hline \multirow{2}{*}{2} & 10.42 & 6.88 & 3.23 & 2.41 & 1.66 & 0.37 & 0.38 & 9.18 & 0.77 & 3.37 & -0.20 & 0.08 & 0.13 & 0.02 & -0.14 & 0.44 & 0.41 & 0.14 & 0.20 & 0.05 & 0.33 & 0.11 \\
\hline & 17.16 & 10.09 & 5.17 & 3.80 & 2.56 & 0.15 & 0.77 & 15.48 & 1.40 & 6.01 & 0.18 & 0.20 & 0.12 & 0.03 & 0.14 & 0.64 & 0.29 & 0.17 & 0.44 & 0.07 & 0.23 & 0.11 \\
\hline \multirow{2}{*}{3} & 37.32 & 59.87 & 4.27 & 3.48 & 1.83 & 0.45 & 0.71 & 47.59 & 0.70 & 1.70 & -0.26 & 0.14 & 0.18 & 0.01 & -0.18 & 0.81 & 0.65 & 0.10 & 0.38 & -0.07 & 0.40 & 0.17 \\
\hline & 63.63 & 102.45 & 5.73 & 5.37 & 2.49 & 0.10 & 1.16 & 81.35 & 0.89 & 2.46 & 0.26 & 0.25 & 0.18 & 0.03 & 0.19 & 1.16 & 0.51 & 0.25 & 0.63 & 0.12 & 0.34 & 0.21 \\
\hline \multirow{2}{*}{4} & 70.94 & 260.10 & 2.53 & 1.50 & 0.78 & 0.55 & 0.74 & 86.44 & 1.80 & 5.53 & -0.27 & 0.17 & 0.16 & 0.03 & -0.18 & 0.90 & 0.97 & 0.21 & 0.39 & 0.03 & 0.45 & 0.20 \\
\hline & 121.45 & 444.69 & 3.92 & 1.96 & 0.78 & 0.27 & 1.53 & 148.97 & 1.31 & 4.54 & 0.26 & 0.42 & 0.16 & 0.05 & 0.19 & 1.41 & 1.64 & 0.73 & 0.87 & 0.09 & 0.44 & 0.24 \\
\hline \multirow{2}{*}{5} & 8.73 & 28.81 & 1.25 & 0.81 & 0.36 & 0.33 & 0.29 & 11.83 & 1.86 & 11.33 & -0.18 & 0.07 & 0.11 & -0.00 & -0.11 & 0.40 & 0.28 & 0.08 & 0.17 & -0.04 & 0.29 & 0.10 \\
\hline & 14.47 & 48.61 & 1.47 & 1.03 & 0.29 & 0.15 & 0.52 & 20.05 & 2.65 & 17.88 & 0.21 & 0.16 & 0.14 & 0.01 & 0.14 & 0.57 & 0.43 & 0.33 & 0.32 & 0.06 & 0.29 & 0.13 \\
\hline \multirow{2}{*}{6} & 7.99 & 13.46 & 2.75 & 1.20 & 0.63 & 0.50 & 0.38 & 10.67 & 1.03 & 3.34 & -0.18 & 0.09 & 0.11 & 0.01 & -0.12 & 0.43 & 0.40 & 0.12 & 0.22 & 0.04 & 0.29 & 0.10 \\
\hline & 13.06 & 22.34 & 4.24 & 1.68 & 0.71 & 0.10 & 0.74 & 18.06 & 1.21 & 3.71 & 0.24 & 0.23 & 0.16 & 0.02 & 0.17 & 0.64 & 0.76 & 0.51 & 0.45 & 0.07 & 0.35 & 0.13 \\
\hline \multirow{2}{*}{7} & 3.41 & 6.16 & 1.39 & 1.16 & 0.45 & 0.55 & 0.08 & 3.81 & 0.91 & 3.53 & -0.15 & -0.02 & 0.10 & -0.01 & -0.09 & 0.19 & 0.72 & 0.34 & 0.03 & 0.04 & 0.23 & 0.07 \\
\hline & 4.74 & 7.90 & 1.53 & 1.17 & 0.23 & 0.19 & 0.41 & 6.59 & 1.77 & 6.04 & 0.18 & 0.21 & 0.13 & 0.01 & 0.12 & 0.39 & 1.02 & 0.58 & 0.30 & 0.07 & 0.29 & 0.09 \\
\hline \multirow{2}{*}{8} & 243.84 & 517.81 & 22.66 & 17.04 & 3.76 & 0.76 & 1.27 & 310.35 & 2.15 & 9.05 & -0.20 & 0.20 & 0.16 & 0.00 & -0.15 & 1.30 & 1.09 & -0.04 & 0.62 & -0.12 & 0.19 & 0.18 \\
\hline & 421.16 & 893.56 & 38.91 & 28.68 & 5.70 & 0.46 & 1.92 & 537.01 & 2.74 & 10.12 & 0.44 & 0.26 & 0.31 & 0.03 & 0.33 & 2.24 & 1.37 & $\begin{array}{ll}0.17 \\
\end{array}$ & 0.90 & 0.16 & 0.64 & 0.36 \\
\hline \multirow{2}{*}{9} & 33.35 & 34.29 & 4.80 & 2.61 & 0.73 & 0.43 & 0.39 & 27.36 & 1.96 & 15.04 & -0.15 & 0.08 & 0.10 & 0.01 & -0.11 & 0.52 & 0.71 & 0.10 & 0.21 & 0.04 & 0.23 & 0.12 \\
\hline & 56.71 & 57.72 & 7.46 & 3.67 & 0.80 & 0.15 & 0.96 & 47.71 & 3.35 & 25.84 & 0.37 & 0.27 & 0.23 & 0.05 & 0.25 & 1.01 & 1.22 & 0.35 & 0.55 & 0.08 & 0.52 & 0.21 \\
\hline \multirow{2}{*}{10} & 1.63 & 4.58 & 0.89 & 0.85 & 0.30 & 0.52 & 0.01 & 1.48 & 1.28 & 7.09 & -0.18 & 0.06 & 0.12 & -0.02 & -0.11 & 0.15 & 1.12 & 0.46 & -0.03 & 0.05 & 0.30 & 0.07 \\
\hline & 1.47 & 5.41 & 0.57 & 0.63 & 0.01 & 0.23 & 0.36 & 2.17 & 1.40 & 7.87 & 0.11 & 0.20 & 0.08 & 0.01 & 0.08 & 0.25 & 1.66 & 0.72 & 0.27 & 0.04 & 0.18 & 0.05 \\
\hline \multirow{2}{*}{11} & 1.77 & 4.23 & 0.88 & 0.96 & 0.32 & 1.02 & 0.07 & 1.80 & 0.61 & 1.73 & -0.08 & 0.03 & 0.07 & -0.00 & -0.07 & 0.14 & 1.24 & 0.52 & 0.02 & 0.06 & 0.07 & 0.06 \\
\hline & 2.14 & 6.63 & 1.07 & 1.10 & 0.16 & 0.91 & 0.29 & 3.14 & 1.77 & 4.11 & 0.34 & 0.18 & 0.23 & 0.01 & 0.23 & 0.31 & 2.81 & 1.16 & 0.23 & 0.05 & 0.56 & 0.17 \\
\hline \multirow{2}{*}{12} & 35.99 & 76.70 & 22.21 & 5.31 & 4.40 & 0.80 & 0.77 & 47.13 & 0.86 & 2.35 & -0.24 & 0.13 & 0.16 & 0.00 & -0.16 & 0.81 & 0.90 & 0.22 & 0.39 & 0.07 & 0.33 & 0.15 \\
\hline & 61.08 & 129.00 & 37.67 & 8.64 & 6.84 & 0.13 & 1.44 & 81.36 & 1.04 & 2.91 & 0.30 & 0.34 & 0.22 & 0.01 & 0.22 & 1.37 & 1.00 & 0.62 & 0.79 & 0.12 & 0.45 & 0.21 \\
\hline \multirow{2}{*}{13} & 39.05 & 89.74 & 14.99 & 5.50 & 0.88 & 0.65 & 0.85 & 39.66 & 1.06 & 6.25 & -0.19 & 0.16 & 0.13 & 0.02 & -0.14 & 0.74 & 0.21 & 0.03 & 0.43 & -0.08 & 0.21 & 0.13 \\
\hline & 67.01 & 154.88 & 25.19 & 9.07 & 0.91 & 0.26 & 1.45 & 68.70 & 2.15 & 11.43 & 0.33 & 0.31 & 0.22 & 0.04 & 0.24 & 1.24 & 0.62 & 0.47 & 0.73 & 0.11 & 0.49 & 0.21 \\
\hline & 3.03 & 12.70 & 3.07 & 1.55 & 0.88 & 0.79 & 0.25 & 3.72 & 0.42 & 2.06 & -0.07 & 0.05 & 0.06 & -0.01 & -0.05 & 0.22 & 0.18 & 0.07 & 0.15 & 0.05 & 0.05 & 0.04 \\
\hline & 4.12 & 21.24 & 4.59 & 1.75 & 0.91 & 0.86 & 0.40 & 6.66 & 1.50 & 4.77 & 0.29 & 0.15 & 0.19 & 0.01 & 0.19 & 0.51 & 0.83 & 0.38 & 0.27 & 0.06 & 0.49 & 0.13 \\
\hline & 2.68 & 11.98 & 2.42 & 1.36 & 0.93 & 0.83 & 0.18 & 4.63 & 1.75 & 6.62 & -0.17 & 0.05 & 0.12 & -0.00 & -0.11 & 0.34 & 2.05 & 0.19 & 0.12 & -0.03 & 0.31 & 0.09 \\
\hline & 3.21 & 19.32 & 3.70 & 1.79 & 0.69 & 0.54 & 0.29 & 6.83 & 2.49 & 9.77 & 0.24 & 0.13 & 0.16 & 0.01 & 0.16 & 0.41 & 3.33 & 0.42 & 0.21 & 0.02 & 0.38 & 0.13 \\
\hline \multirow{2}{*}{16} & 50.15 & 124.37 & 21.62 & 9.47 & 2.52 & 0.43 & 0.73 & 70.08 & 2.44 & 12.69 & -0.19 & 0.19 & 0.13 & 0.00 & -0.13 & 0.79 & 0.51 & -0.04 & 0.42 & -0.04 & 0.29 & 0.13 \\
\hline & 86.20 & 215.09 & 36.99 & 15.85 & 3.93 & 0.19 & 1.28 & 120.92 & 3.01 & 18.31 & 0.29 & 0.36 & 0.19 & 0.02 & 0.20 & 1.36 & 0.83 & 0.21 & 0.74 & 0.05 & 0.42 & 0.21 \\
\hline
\end{tabular}

Table 36: Time domain parameters SESSION 4 - SESSION 1 difference for Acoustic Therapy - TRT 
- EAE therapy (data from 11 patients)

Frequency domain parameters

\begin{tabular}{|c|c|c|c|c|c|c|c|c|c|c|c|c|c|c|c|c|c|c|c|c|c|c|c|}
\hline Channel & & PSDtotal & PSD- $\delta$ & PSD $-\theta$ & PSD- $\alpha$ & PSD- $\beta$ & PSD- $\gamma$ & $\mathrm{P} 1(\mathrm{Xmf})$ & P2 & P3 & P4 & P5(Xfc) & P6 & $\mathrm{P7}(\mathrm{Xrmsf})$ & P8 & P9 & P10 & P11 & P12 & P13 & P14 & MDF & PSDef \\
\hline \multirow{2}{*}{1} & & 40.39 & 45.54 & 5.78 & 1.51 & 0.63 & 0.60 & 0.55 & 20.80 & 0.17 & 0.46 & -0.00 & 0.14 & -0.01 & 0.01 & -0.01 & 0.34 & 0.05 & -0.13 & 0.29 & -0.01 & 0.12 & 0.03 \\
\hline & $\sigma$ & 131.95 & 147.10 & 17.31 & 3.17 & 0.76 & 0.53 & 1.62 & 68.48 & 0.49 & 1.12 & 0.22 & 0.36 & 0.13 & 0.05 & 0.15 & 1.13 & 0.40 & 0.22 & 0.80 & 0.06 & 0.44 & 0.16 \\
\hline \multirow{2}{*}{2} & $\mu$ & 1.94 & 2.09 & 0.80 & 0.52 & 0.46 & 0.43 & 0.02 & 0.78 & 0.11 & 0.60 & 0.18 & -0.02 & 0.07 & -0.00 & 0.08 & -0.05 & 0.03 & -0.00 & 0.03 & 0.02 & 2.17 & -0.05 \\
\hline & J & 3.62 & 3.16 & 1.38 & 0.51 & 0.36 & 0.21 & 0.38 & 2.81 & 0.79 & 1.93 & 0.43 & 0.16 & 0.18 & 0.02 & 0.20 & 0.40 & 0.31 & 0.15 & 0.27 & 0.07 & 6.39 & 0.14 \\
\hline \multirow{2}{*}{3} & 1 & 1.31 & 1.50 & 0.79 & 0.76 & 0.75 & 0.55 & 0.04 & 0.82 & 0.14 & 0.86 & 0.08 & -0.01 & 0.01 & -0.01 & 0.03 & 0.05 & 0.47 & 0.09 & 0.01 & 0.01 & 0.57 & 0.00 \\
\hline & $\sigma$ & 1.13 & 1.57 & 0.81 & 0.71 & 0.68 & 0.28 & 0.42 & 1.72 & 0.74 & 2.74 & 0.56 & 0.17 & 0.26 & 0.02 & 0.28 & 0.34 & 0.94 & 0.33 & 0.30 & 0.14 & 2.49 & 0.13 \\
\hline \multirow{2}{*}{4} & $\mu$ & 0.65 & 0.85 & 0.44 & 0.34 & 0.35 & 0.50 & 0.08 & 0.13 & -0.09 & 0.23 & 0.10 & -0.05 & 0.05 & -0.01 & 0.06 & -0.11 & 0.06 & 0.07 & -0.06 & -0.00 & 0.26 & -0.04 \\
\hline & $\sigma$ & 0.27 & 0.42 & 0.20 & 0.21 & 0.23 & 0.25 & 0.25 & 0.78 & 0.49 & 1.14 & 0.18 & 0.14 & 0.10 & 0.02 & 0.10 & 0.23 & 0.32 & 0.36 & 0.19 & 0.06 & 0.46 & 0.07 \\
\hline \multirow{2}{*}{5} & & 0.48 & 0.62 & 0.45 & 0.35 & 0.30 & 0.41 & 0.05 & 0.16 & -0.10 & 0.14 & 0.08 & -0.03 & 0.04 & -0.00 & 0.05 & -0.08 & 0.08 & 0.04 & -0.04 & 0.00 & 0.24 & -0.03 \\
\hline & & 0.21 & 0.36 & 0.25 & 0.19 & 0.23 & 0.16 & 0.22 & 0.48 & 0.51 & 1.37 & 0.15 & 0.13 & 0.08 & 0.02 & 0.07 & 0.19 & 0.14 & 0.31 & 0.17 & 0.04 & 0.56 & 0.06 \\
\hline \multirow{2}{*}{6} & $\mu$ & 0.71 & 1.15 & 0.42 & 0.42 & 0.31 & 0.46 & 0.09 & 0.09 & -0.03 & 0.64 & 0.11 & -0.06 & 0.05 & -0.01 & 0.06 & -0.12 & 0.07 & 0.07 & 0.07 & 0.00 & 0.97 & -0.04 \\
\hline & $\sigma$ & 0.41 & 1.27 & 0.14 & 0.18 & 0.14 & 0.35 & 0.23 & 1.11 & 0.80 & 2.69 & 0.19 & 0.15 & 0.10 & 0.02 & 0.10 & 0.23 & 0.22 & 0.35 & 0.19 & 0.03 & 2.61 & 0.08 \\
\hline \multirow{2}{*}{7} & & 0.61 & 0.60 & 0.49 & 0.42 & 0.32 & 0.41 & 0.09 & 0.36 & -0.33 & 0.45 & 0.10 & 0.07 & 0.04 & -0.02 & 0.06 & -0.13 & 0.07 & 0.12 & 0.08 & 0.02 & 0.43 & -0.05 \\
\hline & $\sigma$ & 0.24 & 0.25 & 0.50 & 0.18 & 0.17 & 0.34 & 0.24 & 0.57 & 0.28 & 0.48 & 0.19 & 0.15 & 0.09 & 0.02 & 0.11 & 0.23 & 0.24 & 0.36 & 0.20 & 0.05 & 0.92 & 0.07 \\
\hline \multirow{2}{*}{8} & 7 & 1.02 & 0.77 & 0.40 & 0.42 & 1.08 & 1.95 & 0.13 & 0.00 & -0.40 & 0.51 & 0.36 & 0.02 & $\begin{array}{l}0.15 \\
\end{array}$ & -0.01 & 0.17 & -0.12 & 0.07 & -0.03 & 0.08 & 0.01 & 1.40 & -0.08 \\
\hline & $\sigma \sigma$ & 0.62 & 0.69 & 0.22 & 0.25 & 1.00 & 1.89 & 0.55 & 1.00 & 0.40 & 0.57 & 0.85 & 0.22 & 0.37 & 0.02 & 0.38 & 0.30 & 0.93 & 0.56 & 0.38 & 0.16 & 3.97 & 0.12 \\
\hline \multirow{2}{*}{9} & & 0.72 & 1.84 & 0.49 & 0.52 & 0.34 & 0.50 & 0.08 & 0.09 & -0.14 & 0.19 & 0.05 & -0.06 & 0.02 & -0.01 & 0.03 & -0.08 & 0.07 & 0.19 & -0.07 & -0.01 & 0.12 & -0.02 \\
\hline & $\mid \sigma$ & 0.26 & 2.57 & 0.18 & 0.27 & 0.16 & 0.18 & 0.28 & 0.82 & 0.60 & 1.47 & 0.15 & 0.18 & 0.09 & 0.02 & 0.09 & 0.24 & 0.44 & 0.54 & 0.22 & 0.04 & 0.33 & 0.06 \\
\hline \multirow{2}{*}{10} & & 0.55 & 0.63 & $\begin{array}{l}0.43 \\
\end{array}$ & 0.45 & 0.32 & 0.37 & 0.07 & 0.31 & -0.33 & 0.44 & 0.14 & -0.04 & 0.07 & -0.00 & 0.07 & -0.13 & 0.14 & 0.04 & -0.06 & 0.00 & 0.34 & -0.05 \\
\hline & $\sigma \sigma$ & 0.29 & 0.50 & 0.21 & 0.28 & 0.19 & 0.21 & 0.22 & 0.61 & 0.35 & 0.51 & 0.21 & 0.13 & 0.10 & 0.01 & 0.11 & 0.21 & 0.22 & 0.32 & 0.17 & 0.05 & 0.55 & 0.07 \\
\hline \multirow{2}{*}{11} & & 0.78 & 1.31 & 0.35 & 0.37 & 0.50 & 0.70 & 0.12 & 0.15 & 0.08 & 1.85 & 0.17 & -0.08 & 0.07 & -0.02 & 0.09 & -0.14 & 0.39 & 0.22 & -0.11 & 0.01 & 0.86 & -0.05 \\
\hline & $\sigma$ & 0.50 & 1.44 & 0.24 & 0.22 & 0.43 & 0.39 & 0.39 & 1.14 & 1.01 & 6.05 & 0.40 & 0.20 & 0.20 & 0.03 & 0.24 & 0.28 & 1.55 & 0.59 & 0.30 & 0.09 & 1.99 & 0.11 \\
\hline \multirow{2}{*}{12} & & 0.74 & 0.71 & $\begin{array}{l}0.57 \\
\end{array}$ & 0.50 & 0.61 & 1.57 & 0.07 & 0.03 & -0.22 & 0.18 & 0.24 & 0.02 & 0.11 & -0.00 & 0.12 & -0.08 & 0.19 & 0.05 & 0.04 & 0.01 & 0.72 & -0.05 \\
\hline & & 0.67 & 0.38 & 0.37 & 0.30 & 0.69 & 2.46 & 0.41 & 0.90 & 0.38 & 0.83 & 0.62 & 0.18 & 0.27 & 0.02 & 0.31 & 0.28 & 0.46 & 0.41 & 0.28 & 0.09 & 2.02 & 0.12 \\
\hline \multirow{2}{*}{13} & & 0.56 & 0.56 & 0.49 & 0.53 & 0.43 & 0.48 & 0.01 & 0.18 & -0.25 & 0.28 & 0.10 & 0.01 & 0.06 & 0.00 & 0.05 & -0.07 & 0.18 & -0.04 & 0.01 & 0.01 & 0.20 & -0.03 \\
\hline & & 0.24 & 0.31 & 0.33 & 0.28 & 0.21 & 0.34 & 0.19 & 0.46 & 0.36 & 0.60 & 0.14 & 0.12 & 0.07 & 0.02 & 0.08 & 0.18 & 0.19 & 0.29 & 0.15 & 0.03 & 0.37 & 0.06 \\
\hline \multirow{2}{*}{14} & & 0.72 & 1.31 & 0.53 & 0.63 & 0.43 & 0.75 & 0.07 & 0.16 & -0.21 & 0.18 & 0.27 & -0.04 & 0.12 & -0.01 & $\begin{array}{l}0.14 \\
\end{array}$ & -0.14 & 0.07 & 0.05 & -0.07 & 0.04 & 2.37 & -0.07 \\
\hline & T & 0.28 & 1.68 & 0.35 & 0.52 & 0.21 & 0.76 & 0.41 & 0.73 & 0.37 & 0.75 & 0.52 & 0.21 & 0.22 & 0.03 & 0.26 & 0.32 & 0.47 & 0.41 & 0.31 & 0.11 & 6.53 & 0.13 \\
\hline \multirow{2}{*}{15} & $d$ & 0.70 & 0.80 & $\begin{array}{ll}0.49 \\
\end{array}$ & $\begin{array}{l}0.61 \\
\end{array}$ & 0.51 & 0.77 & 0.06 & 0.03 & -0.14 & 0.11 & 0.17 & 0.02 & 0.08 & -0.00 & 0.09 & -0.09 & $\begin{array}{l}0.39 \\
\end{array}$ & -0.07 & 0.04 & 0.00 & 0.55 & -0.06 \\
\hline & & 0.33 & 0.65 & 0.41 & 0.30 & 0.39 & 0.72 & 0.36 & 0.75 & 0.45 & 1.06 & 0.21 & 0.18 & 0.09 & 0.02 & 0.11 & 0.25 & 0.25 & 0.29 & 0.27 & 0.05 & 1.00 & 0.07 \\
\hline \multirow{2}{*}{16} & & 0.64 & 0.75 & 0.52 & 0.54 & 0.44 & 0.57 & 0.03 & 0.28 & -0.30 & 0.29 & 0.19 & -0.02 & 0.10 & -0.01 & $\begin{array}{l}0.10 \\
\end{array}$ & -0.13 & 0.28 & -0.04 & 0.03 & 0.00 & 0.74 & -0.06 \\
\hline & $\sigma$ & 0.23 & 0.46 & 0.40 & 0.31 & 0.27 & 0.47 & 0.29 & 0.54 & 0.43 & 0.76 & 0.34 & 0.15 & 0.16 & 0.02 & 0.18 & 0.23 & 0.25 & 0.28 & 0.22 & 0.04 & 1.40 & 0.09 \\
\hline
\end{tabular}

Table 37: Frequency domain parameters SESSION 4 - SESSION 1 difference for Acoustic Therapy - EAE 
$\underline{\text { Time domain parameters }}$

\begin{tabular}{|c|c|c|c|c|c|c|c|c|c|c|c|c|c|c|c|c|c|c|c|c|c|c|}
\hline Channe & & Mean & MeanAbs & Median & Max & $\min$ & Peak & $\mathrm{SSI}$ & IEEG & WL & MMAV1 & MMAV2 & STD DEV & VAR & ROOT & RMS & CREST & SKEWNESS & \begin{tabular}{|l|} 
KURTOSIS \\
\end{tabular} & CLEARANCE & SHAPE & IMPULSE \\
\hline \multirow{2}{*}{1} & & 12.19 & $\begin{array}{ll}5.94 \\
\end{array}$ & 15.88 & 5.51 & 4.00 & 5.67 & 391.39 & 5.94 & 0.34 & 6.03 & 4.16 & \begin{tabular}{|l|}
5.43 \\
\end{tabular} & 308.12 & 6.18 & 6.08 & 0.13 & \begin{tabular}{|l|}
-1.48 \\
\end{tabular} & \begin{tabular}{|l|}
0.10 \\
\end{tabular} & \begin{tabular}{|l|}
0.24 \\
\end{tabular} & \begin{tabular}{|l|l|}
0.04 \\
\end{tabular} & \begin{tabular}{|l|}
0.20 \\
\end{tabular} \\
\hline & & 48.53 & 19.03 & 59.42 & 17.59 & 11.48 & 17.54 & $1,295.48$ & 19.03 & 0.46 & 19.31 & 12.80 & 17.16 & $1,019.23$ & 19.89 & 19.40 & 0.31 & 2.81 & 0.21 & 0.52 & 0.07 & 0.42 \\
\hline \multirow{2}{*}{2} & & 2.32 & 0.41 & 0.41 & 0.45 & 0.39 & 0.49 & 3.99 & 0.41 & 0.07 & 0.39 & 0.16 & 0.37 & 3.21 & 0.40 & 0.45 & 0.11 & 3.05 & 0.09 & 0.12 & 0.01 & 0.12 \\
\hline & & 8.50 & 1.62 & 3.57 & 1.83 & 1.44 & 1.83 & 12.23 & 1.62 & 0.25 & 1.61 & 1.02 & 1.60 & 10.06 & 1.57 & 1.79 & 0.25 & 4.13 & 0.33 & 0.28 & 0.06 & 0.27 \\
\hline \multirow{2}{*}{3} & & 15.93 & 0.38 & 105.94 & 0.33 & 0.27 & 0.29 & 1.95 & 0.38 & 0.02 & 0.36 & 0.36 & 0.42 & 1.98 & 0.36 & 0.42 & 0.04 & 6.02 & 0.11 & 0.01 & 0.02 & 0.02 \\
\hline & & 50.24 & 0.99 & 362.57 & 0.82 & 0.77 & 0.75 & 4.34 & 0.99 & 0.35 & 0.94 & 0.89 & 1.02 & 4.36 & 0.95 & 1.02 & 0.16 & 18.20 & 0.21 & 0.23 & 0.06 & 0.20 \\
\hline \multirow{2}{*}{4} & & 3.37 & 0.06 & 3.08 & 0.03 & 0.11 & 0.01 & 0.24 & 0.06 & 0.07 & 0.06 & 0.05 & 0.07 & 0.05 & 0.09 & 0.04 & 0.02 & 3.20 & 0.07 & 0.02 & -0.01 & 0.02 \\
\hline & $\sigma \sigma$ & 4.63 & 0.50 & 5.39 & 0.31 & 0.31 & 0.32 & 1.01 & 0.50 & 0.30 & 0.49 & 0.46 & 0.29 & 0.55 & 0.57 & 0.42 & 0.21 & 10.34 & 0.23 & 0.27 & 0.05 & 0.25 \\
\hline \multirow{2}{*}{5} & & 0.14 & 0.07 & 1.44 & 0.06 & 0.01 & 0.01 & 0.01 & 0.07 & 0.05 & 0.09 & 0.10 & 0.07 & 0.02 & 0.07 & 0.06 & 0.08 & 2.14 & 0.01 & 0.08 & -0.00 & 0.08 \\
\hline & $\sigma$ & 4.39 & 0.35 & 29.38 & 0.41 & 0.36 & 0.42 & 0.58 & 0.35 & 0.26 & 0.33 & 0.23 & 0.35 & 0.57 & 0.34 & 0.36 & 0.13 & 6.97 & 0.14 & 0.14 & 0.02 & 0.14 \\
\hline \multirow{2}{*}{6} & & 1.31 & 0.10 & 25.96 & 0.14 & 0.11 & 0.08 & 0.05 & 0.10 & 0.05 & 0.12 & 0.14 & 0.11 & 0.06 & 0.10 & 0.10 & 0.13 & 9.27 & 0.18 & 0.19 & 0.02 & 0.17 \\
\hline & $\sigma$ & 1.13 & 0.42 & 93.33 & 0.43 & 0.29 & 0.39 & 0.69 & 0.42 & 0.27 & 0.44 & 0.53 & 0.39 & 0.66 & 0.43 & 0.40 & 0.35 & 28.03 & 0.66 & 0.49 & 0.05 & 0.43 \\
\hline \multirow{2}{*}{7} & & 0.73 & 0.32 & 1.06 & 0.30 & 0.12 & 0.25 & 0.51 & 0.32 & 0.01 & 0.32 & 0.19 & 0.32 & 0.50 & 0.32 & 0.33 & 0.13 & 0.83 & 0.12 & 0.12 & -0.01 & 0.12 \\
\hline & $\mid \sigma$ & 0.83 & 0.21 & 1.03 & 0.27 & 0.31 & 0.24 & 0.28 & 0.21 & 0.28 & 0.21 & 0.35 & 0.20 & 0.27 & 0.21 & 0.21 & 0.19 & 0.72 & 0.19 & 0.24 & 0.05 & 0.22 \\
\hline \multirow{2}{*}{8} & & 0.37 & 0.20 & 33.15 & 0.14 & 0.15 & 0.09 & 0.78 & 0.20 & 0.47 & 0.20 & 0.27 & 0.18 & 0.78 & 0.20 & 0.19 & 0.02 & 4.11 & 0.04 & 0.02 & -0.01 & 0.02 \\
\hline & To & 16.91 & 0.63 & 110.82 & 0.52 & 0.60 & 0.51 & 1.55 & 0.63 & 0.74 & 0.65 & 0.77 & 0.64 & 1.56 & 0.64 & 0.63 & 0.20 & 8.30 & 0.15 & 0.26 & 0.05 & 0.23 \\
\hline \multirow[b]{2}{*}{9} & & 10.26 & 0.09 & 11.58 & 0.13 & 0.18 & 0.17 & 0.02 & 0.09 & 0.00 & 0.09 & 0.04 & 0.12 & 0.05 & 0.09 & 0.11 & 0.04 & 1.20 & 0.03 & 0.04 & -0.01 & 0.04 \\
\hline & $\sigma$ & 29.36 & 0.50 & 61.66 & 0.46 & 0.44 & 0.45 & 1.11 & 0.50 & 0.39 & 0.51 & 0.55 & 0.44 & 0.92 & 0.51 & 0.49 & 0.22 & 1.56 & 0.16 & 0.24 & 0.02 & 0.23 \\
\hline \multirow{2}{*}{10} & & 0.63 & 0.12 & 23.69 & 0.12 & 0.05 & 0.10 & 0.14 & 0.12 & 0.08 & 0.13 & 0.13 & 0.11 & 0.13 & 0.12 & 0.11 & 0.05 & 2.35 & 0.07 & 0.09 & 0.01 & 0.07 \\
\hline & 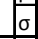 & 1.29 & 0.29 & 82.58 & 0.32 & 0.33 & 0.27 & 0.52 & 0.29 & 0.22 & 0.31 & 0.37 & 0.28 & 0.52 & 0.30 & 0.28 & 0.28 & 3.31 & 0.39 & 0.38 & 0.05 & 0.34 \\
\hline \multirow{2}{*}{11} & & 1.33 & 0.22 & 0.77 & 0.33 & 0.05 & 0.16 & 0.32 & 0.22 & 0.10 & 0.24 & 0.27 & 0.23 & 0.33 & 0.22 & 0.23 & 0.18 & 0.41 & 0.12 & 0.32 & 0.03 & 0.25 \\
\hline & & 2.24 & 0.31 & 2.94 & 0.19 & 0.32 & 0.23 & 0.44 & 0.31 & 0.45 & 0.33 & 0.34 & 0.28 & 0.42 & 0.32 & 0.29 & 0.41 & 5.23 & 0.42 & 0.92 & 0.13 & 0.69 \\
\hline \multirow{2}{*}{12} & & 2.00 & 0.02 & 0.78 & 0.06 & 0.20 & 0.04 & 0.14 & 0.02 & 0.34 & 0.01 & 0.02 & 0.01 & 0.13 & 0.02 & 0.02 & 0.01 & 0.82 & 0.05 & 0.02 & -0.01 & 0.01 \\
\hline & & 4.16 & 0.33 & 5.34 & 0.39 & 0.65 & 0.43 & 0.79 & 0.33 & 0.68 & 0.34 & 0.51 & 0.35 & 0.79 & 0.32 & 0.35 & 0.21 & 2.53 & 0.20 & 0.29 & 0.06 & 0.26 \\
\hline \multirow[b]{2}{*}{13} & & 0.94 & 0.06 & 3.85 & 0.20 & 0.07 & 0.16 & 0.23 & 0.06 & 0.17 & 0.06 & 0.04 & 0.06 & 0.23 & 0.05 & 0.07 & 0.11 & 0.43 & 0.12 & 0.12 & 0.01 & 0.12 \\
\hline & & 4.73 & 0.32 & 15.47 & 0.46 & 0.28 & 0.40 & 0.63 & 0.32 & 0.33 & 0.33 & 0.32 & 0.32 & 0.65 & 0.32 & 0.32 & 0.19 & 2.68 & 0.37 & 0.21 & 0.03 & 0.20 \\
\hline \multirow{2}{*}{14} & & 1.39 & 0.13 & 11.47 & 0.02 & 0.04 & 0.05 & 0.05 & 0.13 & 0.18 & 0.13 & 0.11 & 0.12 & 0.04 & 0.13 & 0.13 & 0.32 & 0.33 & 0.56 & 0.44 & 0.02 & 0.40 \\
\hline & & 5.07 & 0.45 & 35.62 & 0.46 & 0.42 & 0.45 & 0.84 & 0.45 & 0.33 & 0.47 & 0.50 & 0.45 & 0.83 & 0.45 & 0.46 & 0.69 & 5.92 & 1.89 & 1.10 & 0.09 & 0.97 \\
\hline \multirow{2}{*}{15} & & 0.96 & 0.00 & 19.97 & 0.00 & 0.20 & 0.13 & 0.10 & 0.00 & 0.25 & 0.01 & 0.06 & 0.01 & 0.10 & 0.00 & 0.00 & 0.16 & 4.26 & 0.14 & 0.22 & 0.02 & 0.19 \\
\hline & & 6.44 & 0.35 & 88.07 & 0.36 & 0.42 & 0.34 & 0.66 & 0.35 & 0.51 & 0.34 & 0.43 & 0.32 & 0.65 & 0.37 & 0.32 & 0.22 & 5.60 & 0.40 & 0.43 & 0.10 & 0.34 \\
\hline \multirow{2}{*}{16} & & 3.40 & 0.05 & 1.75 & 0.14 & 0.08 & 0.11 & 0.04 & 0.05 & 0.21 & 0.05 & 0.03 & 0.08 & 0.03 & 0.04 & 0.07 & 0.02 & 1.40 & 0.00 & 0.00 & -0.02 & 0.01 \\
\hline & $\sigma$ & 7.63 & 0.44 & 4.41 & 0.42 & 0.39 & 0.27 & 1.04 & 0.44 & 0.37 & 0.42 & 0.54 & 0.44 & 1.05 & 0.44 & 0.43 & 0.22 & 0.58 & 0.14 & 0.21 & 0.04 & 0.22 \\
\hline
\end{tabular}

Table 38: Time domain parameters SESSION 4 - SESSION 1 difference for Acoustic Therapy - EAE 
ANNEX AB: GRAND AVERAGE ERP OF 16 CHANNELS FROM ALL PATIENTS' SESSION 1 AND SESSION 4 OF EACH ACOUSTIC THERAPY

$\underline{\text { Session } 1 \text { Binaural } f_{0}}$
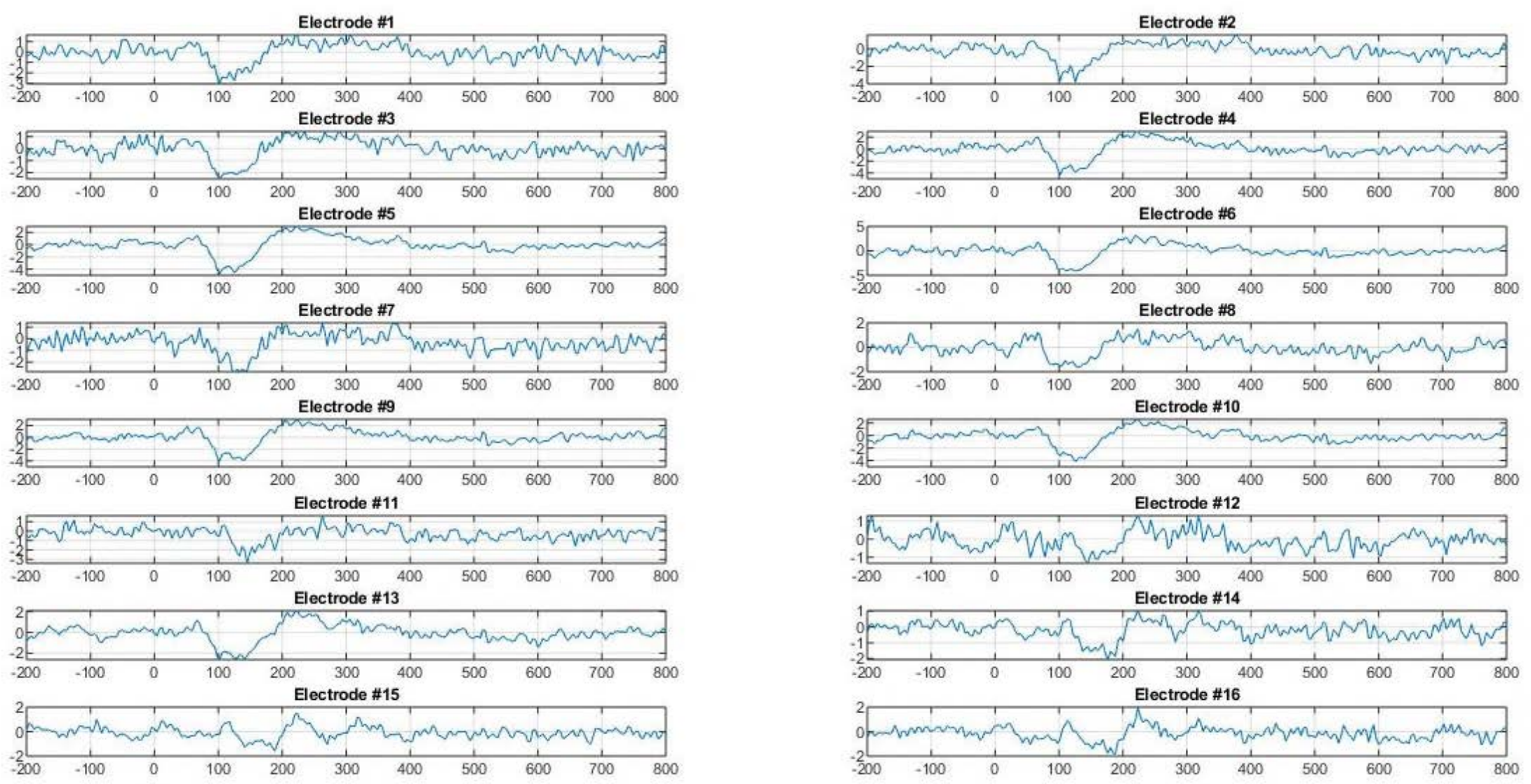

Figure 30: GAv ERP of 16 channels from all patients using Binaural $f_{0}$ Session 1 
Session 4 Binaural $\mathrm{f}_{0}$

$$
\text { (10) }
$$

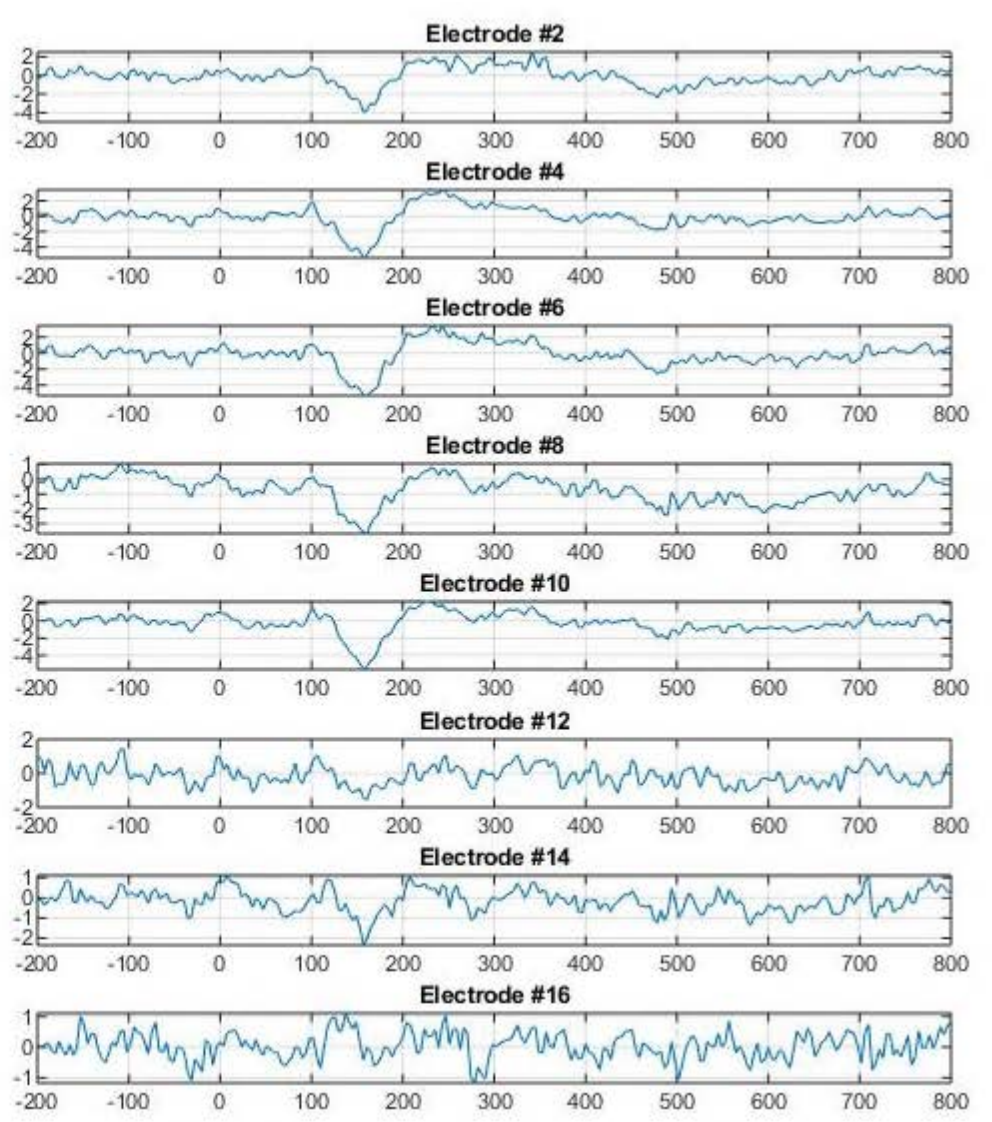

Figure 31: GAv ERP of 16 channels from all patients using Binaural $f_{0}$ Session 4 


\section{Session 1 Binaural $396 \mathrm{~Hz}$}

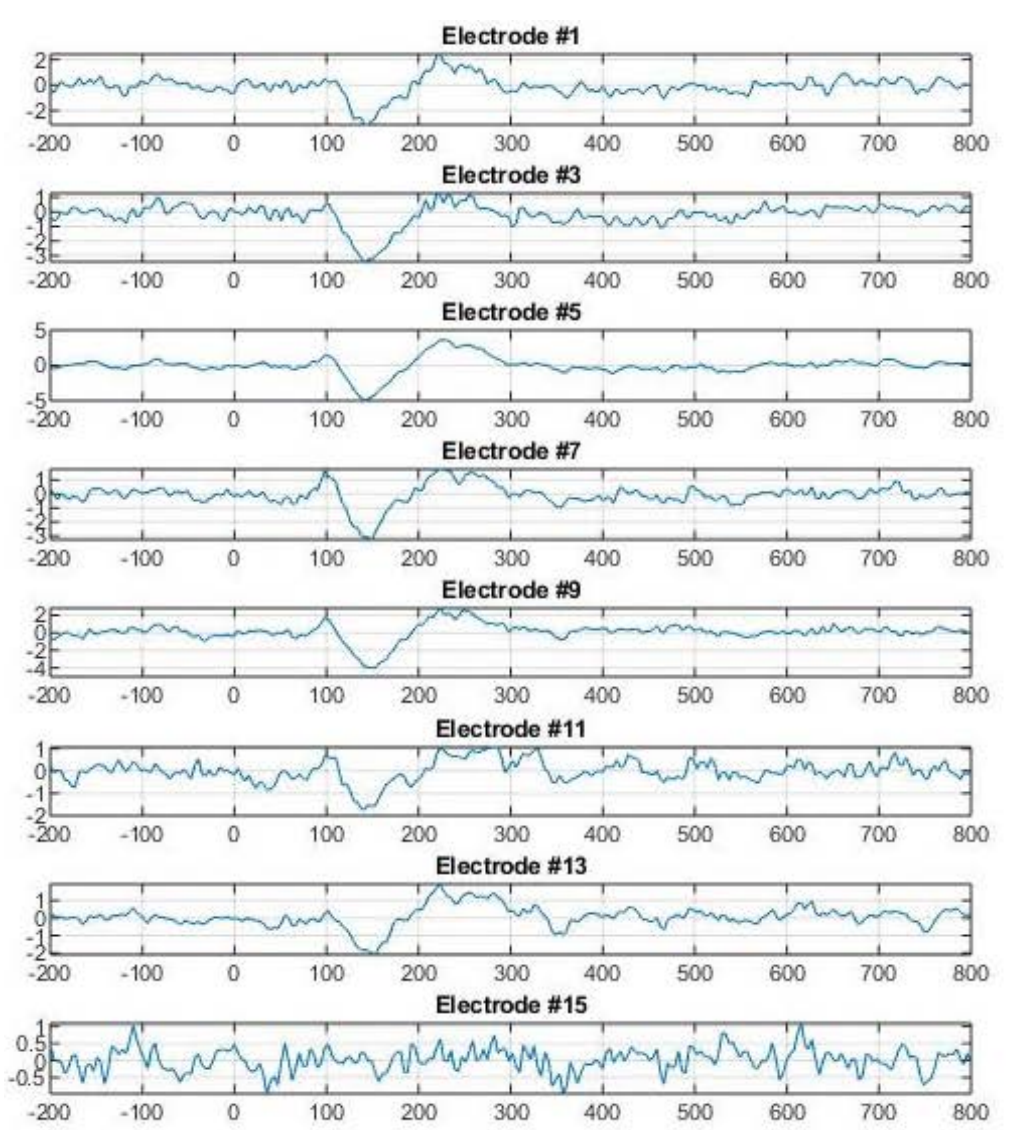

Electrode \#2
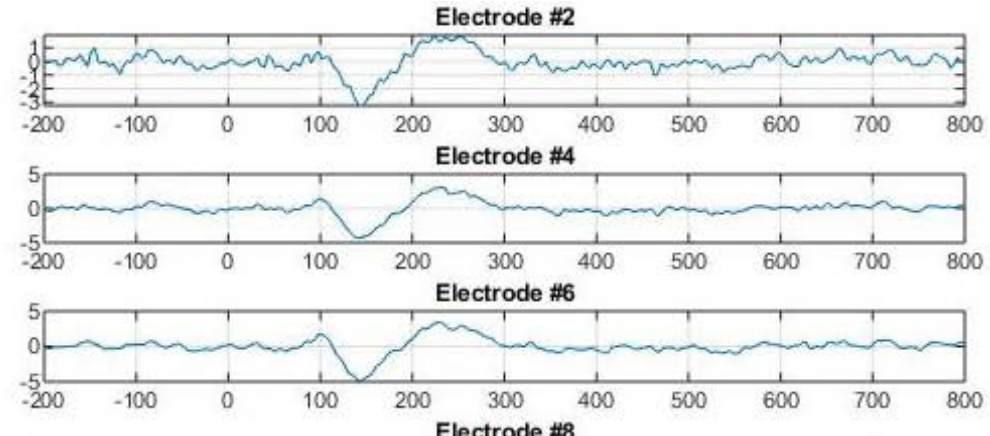

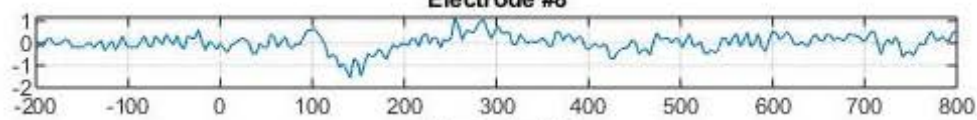

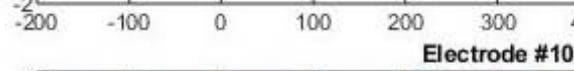

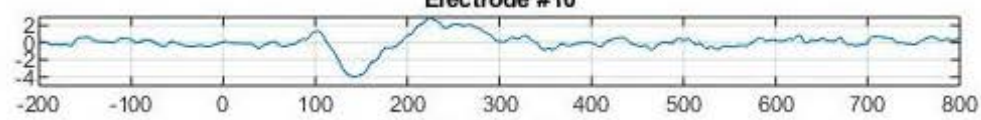

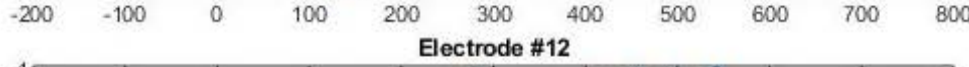

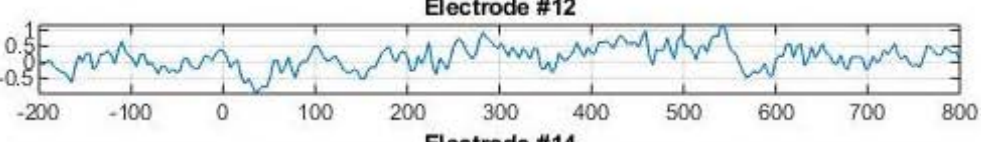

-200 Electrode \#16

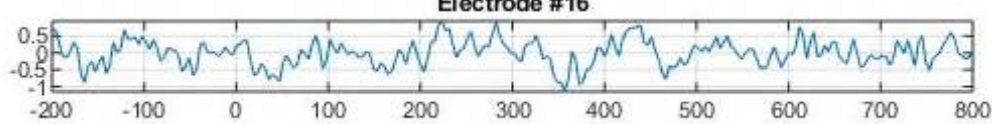

Figure 32: GAv ERP of 16 channels from all patients using Binaural $396 \mathrm{~Hz}$ Session 1 
Session 4 Binaural $396 \mathrm{~Hz}$

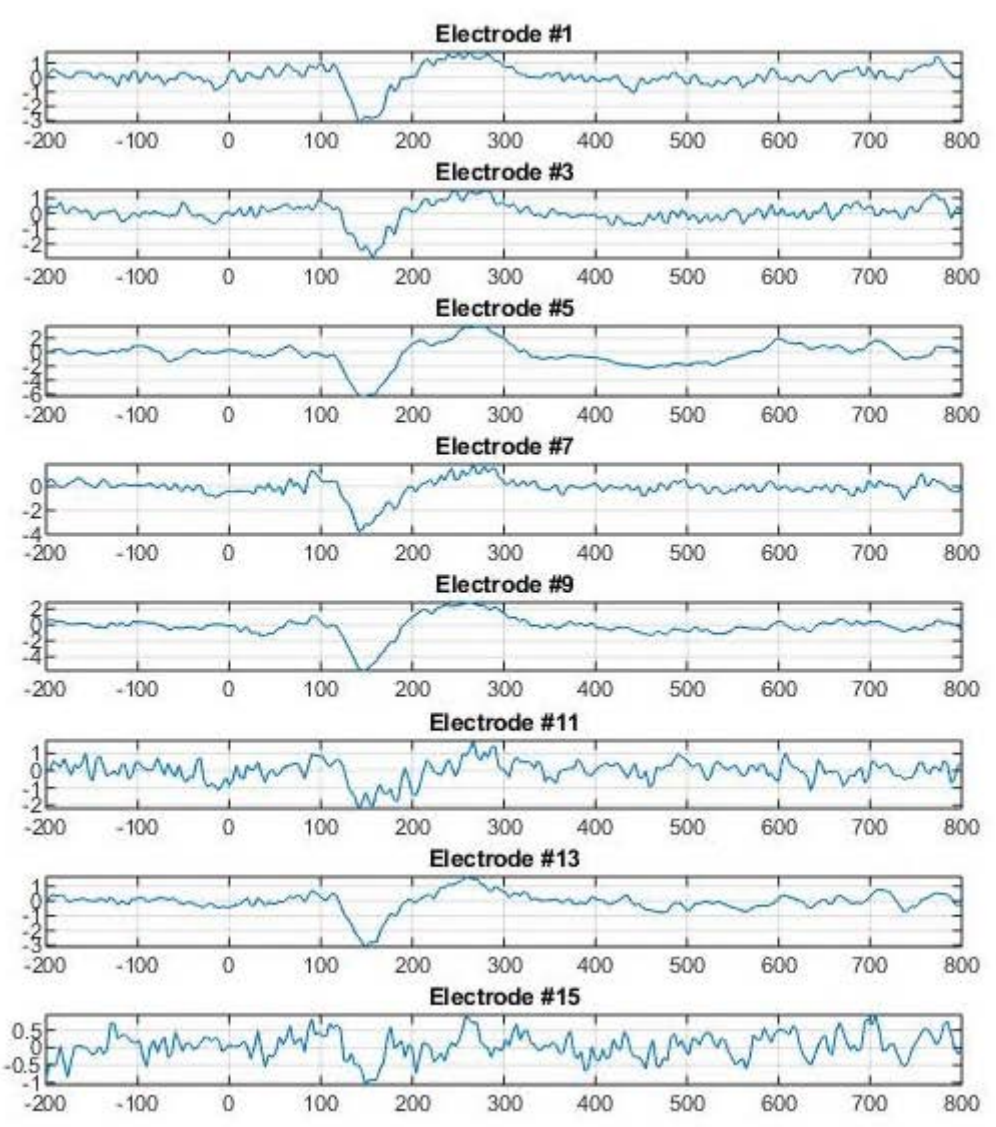

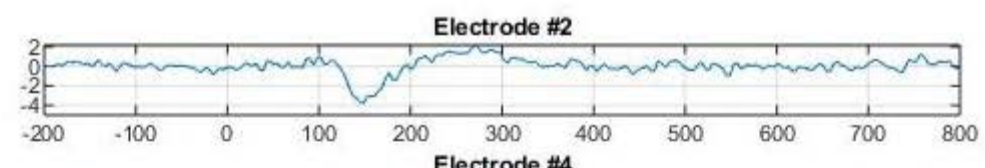
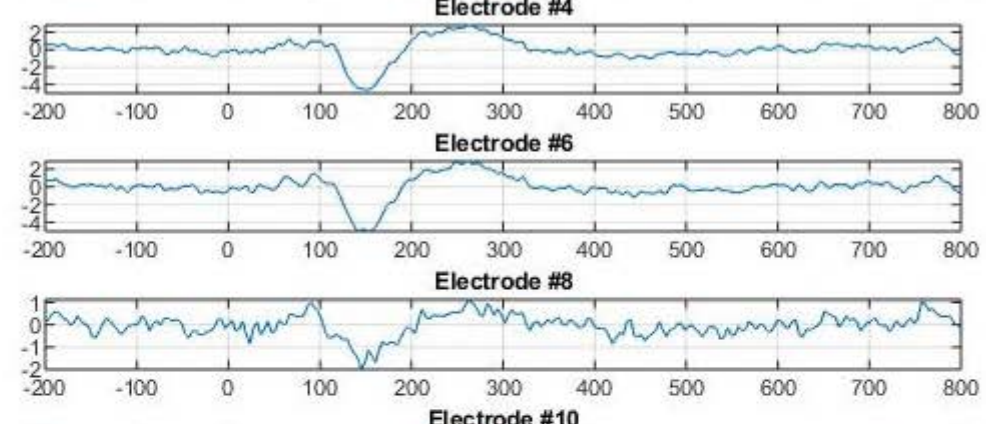

:
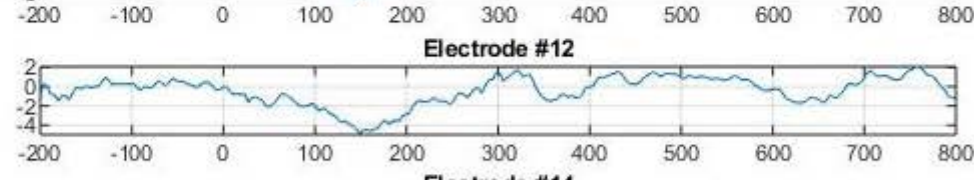

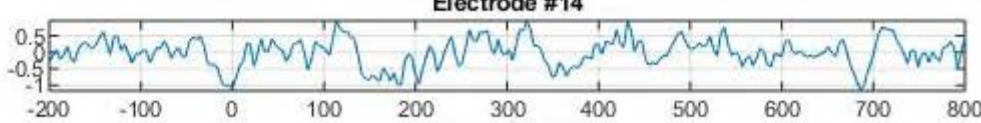

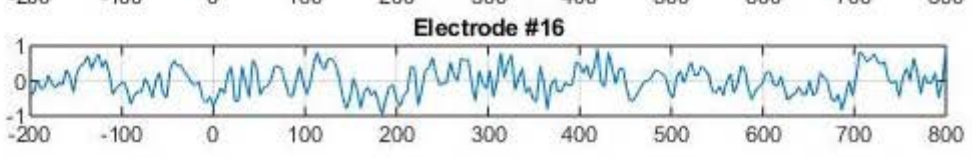

Figure 33: GAv ERP of 16 channels from all patients using Binaural $396 \mathrm{~Hz}$ Session 4 


\section{$\underline{\text { Session } 1 \text { TRT }}$}

$$
\text { (2000 }
$$

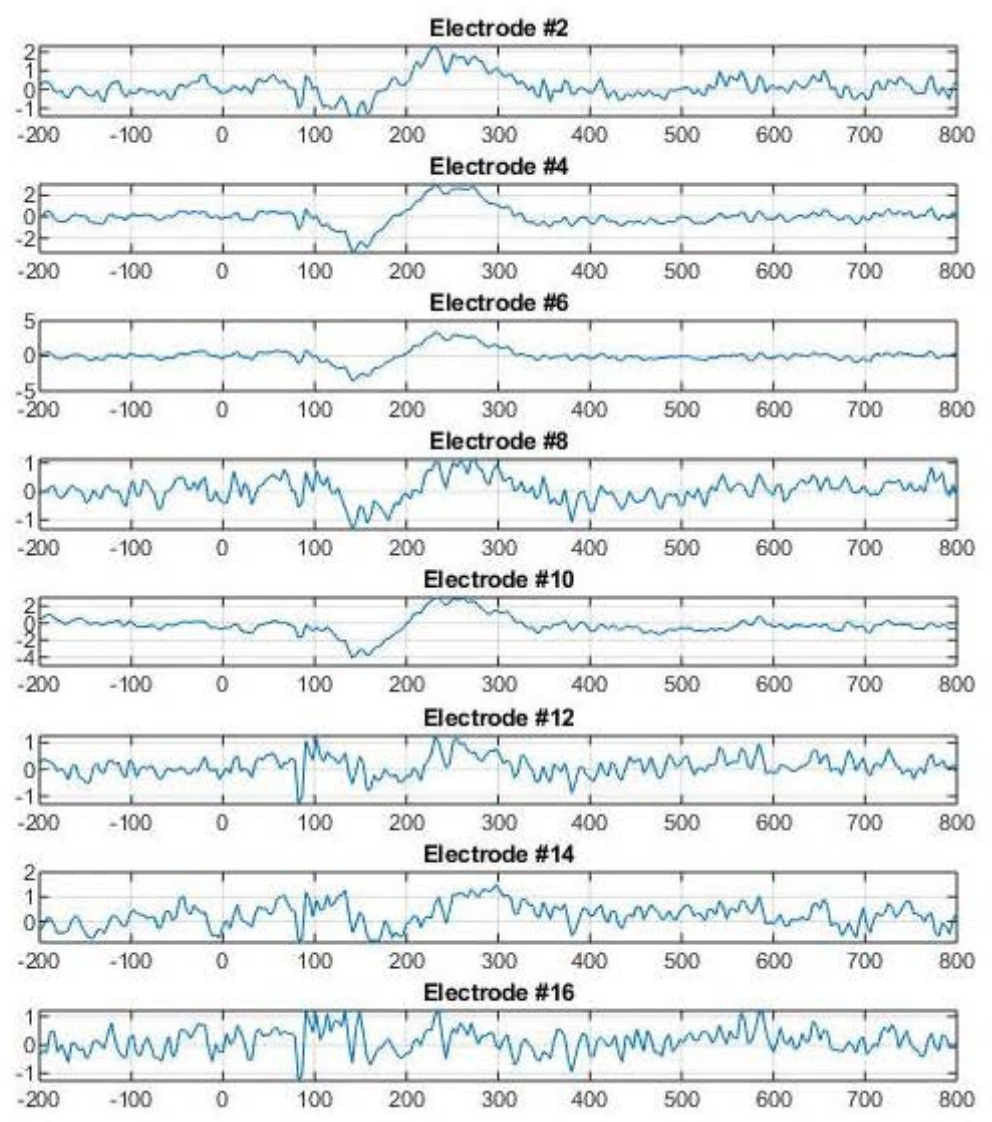

Figure 34: GAv ERP of 16 channels from all patients using TRT Session 1 


\section{Session 4 TRT}
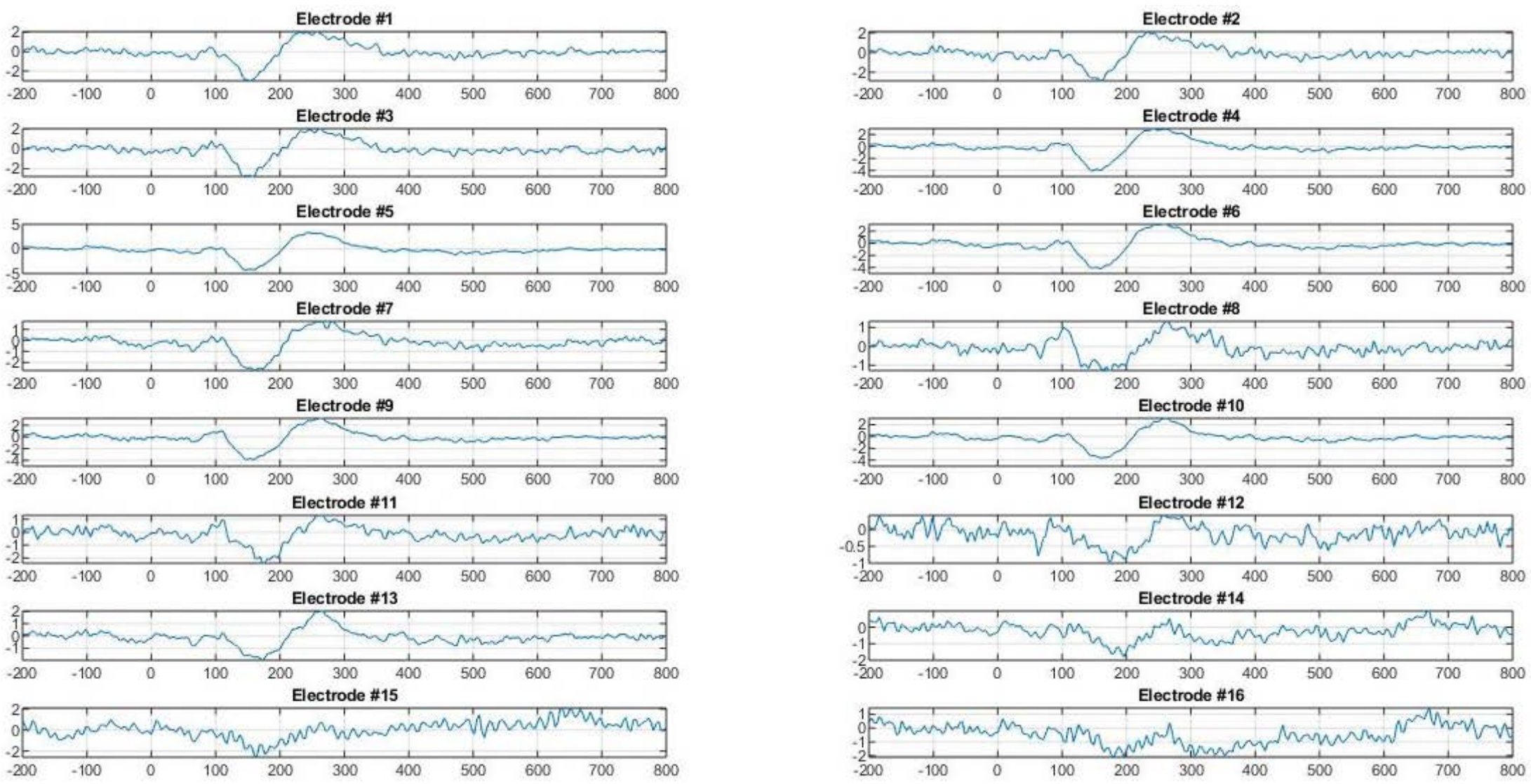

Figure 35: GAv ERP of 16 channels from all patients using TRT Session 4 


\section{Session $1 \mathrm{EAE}$}

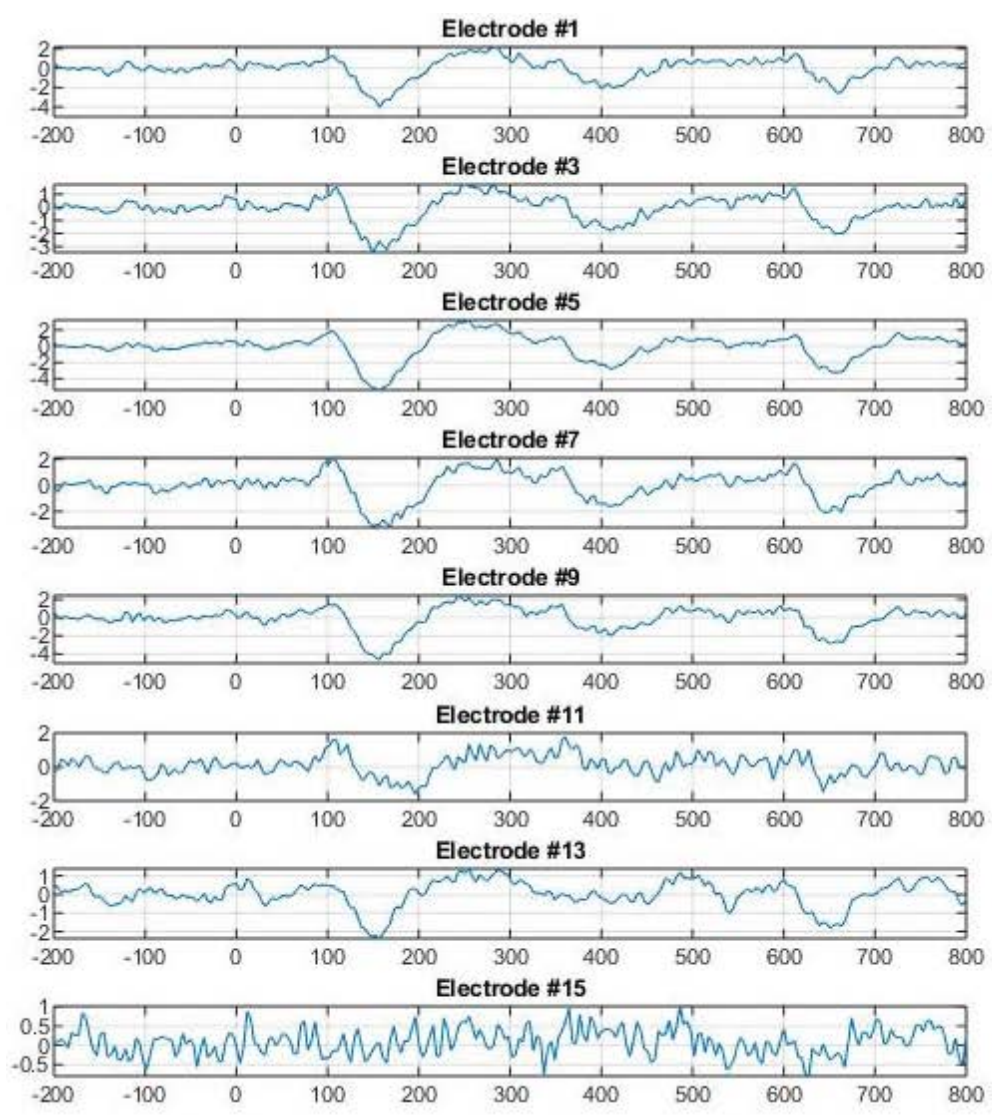

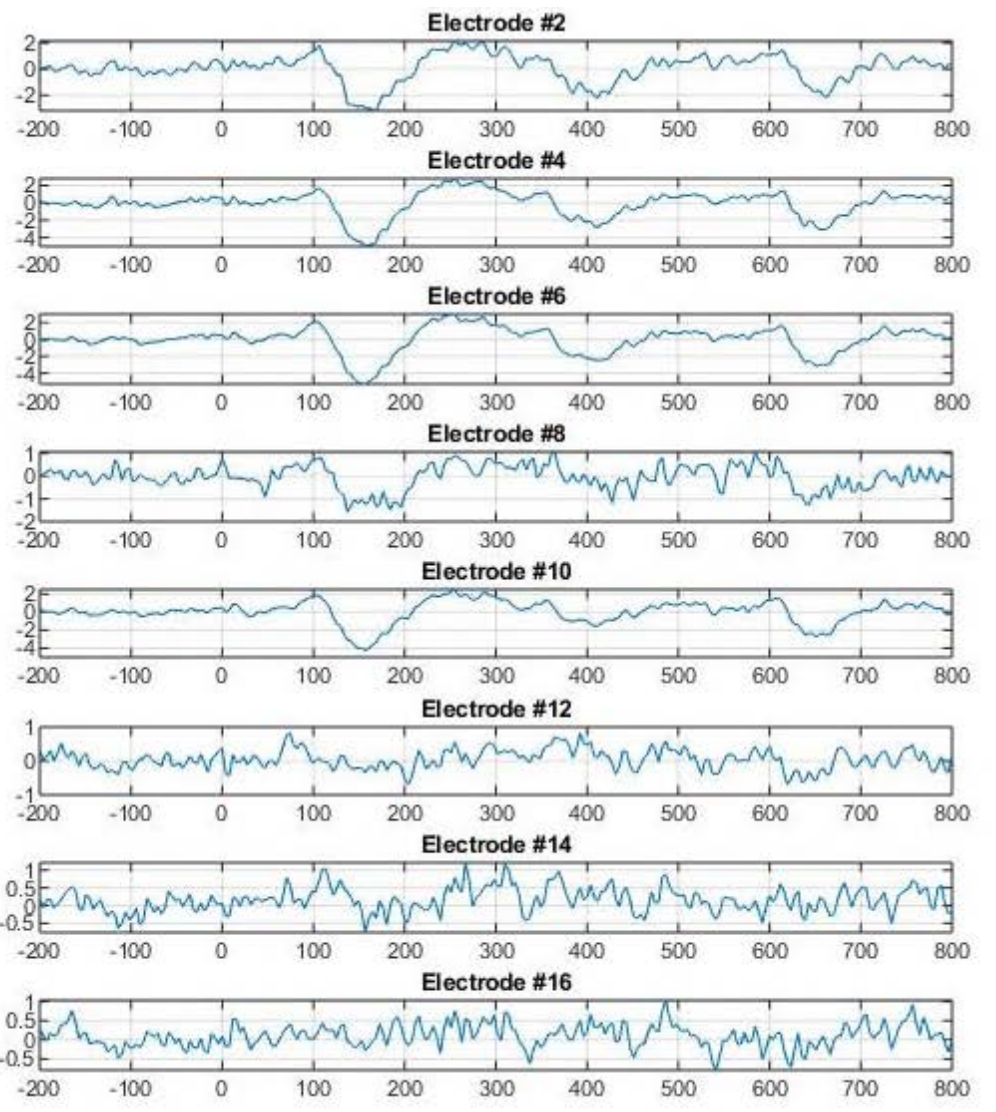

Figure 36: GAv ERP of 16 channels from all patients using EAE Session 1 
Session 4 EAE
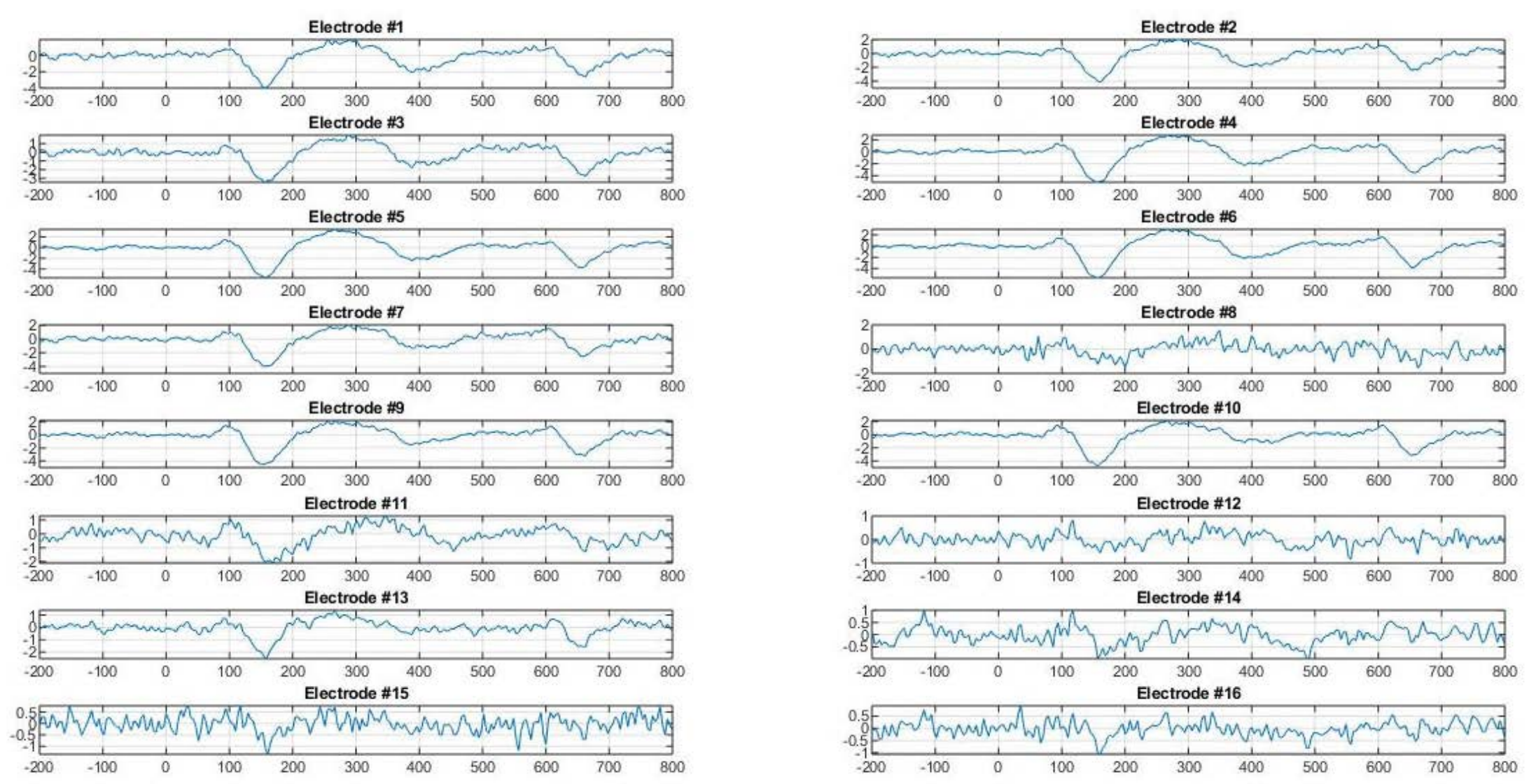

Figure 37: GAv ERP of 16 channels from all patients using EAE Session 4 
ANNEX AC: EXAMPLE OF PATIENT PER ACOUSTIC THERAPY FOR ERPS PRINCIPAL AND INDEPENDENT COMPONENT ANALYSIS

Session 1 Patient 3 - Binaural $\mathrm{f}_{0}$

Session 4 Patient 3 - Binaural $\mathrm{f}_{0}$

Independent components analysis
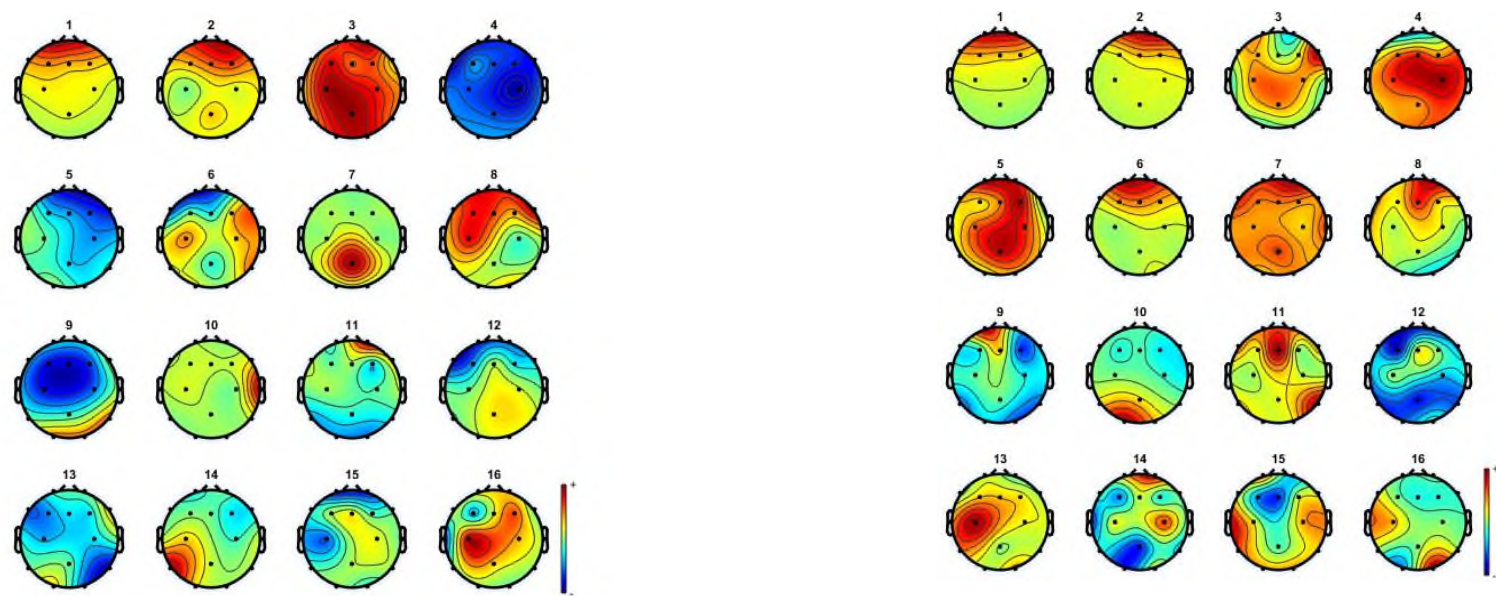

Principal components analysis at P1
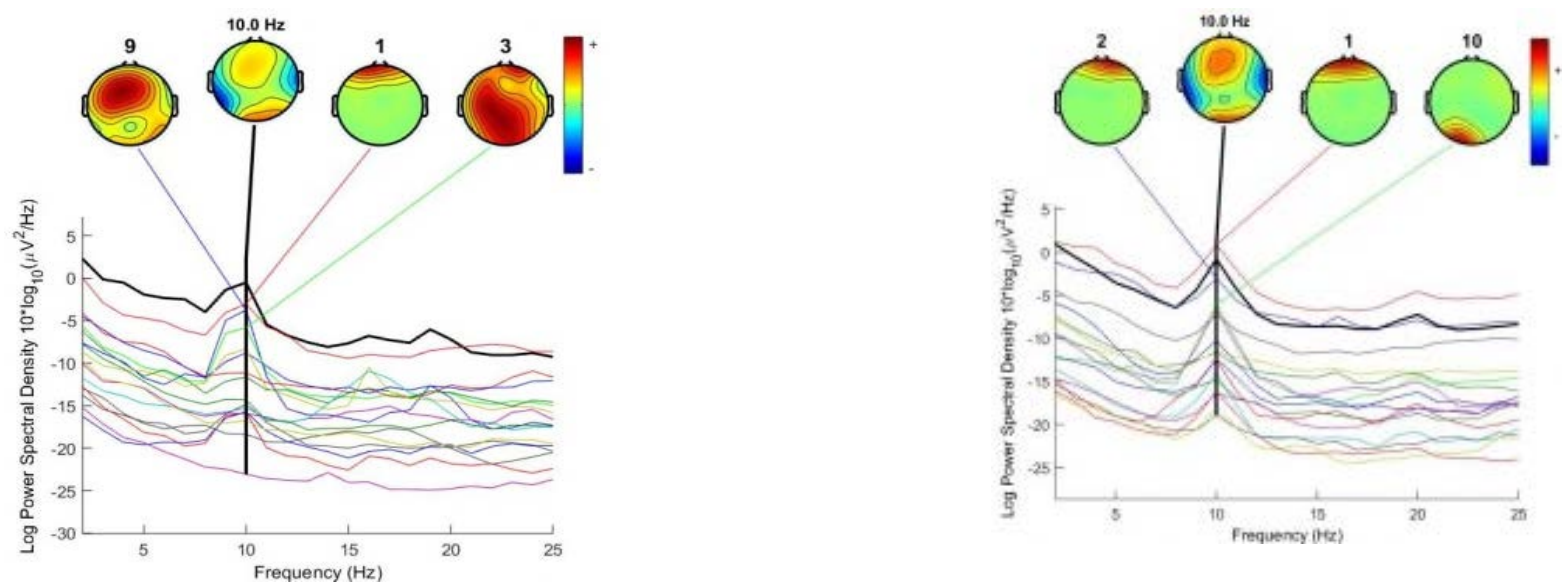

Figure 38: ERPs Principal and Independent Component Analysis for Patient 3 - Binaural $f_{0}$ (Session 1 and 4 ) 


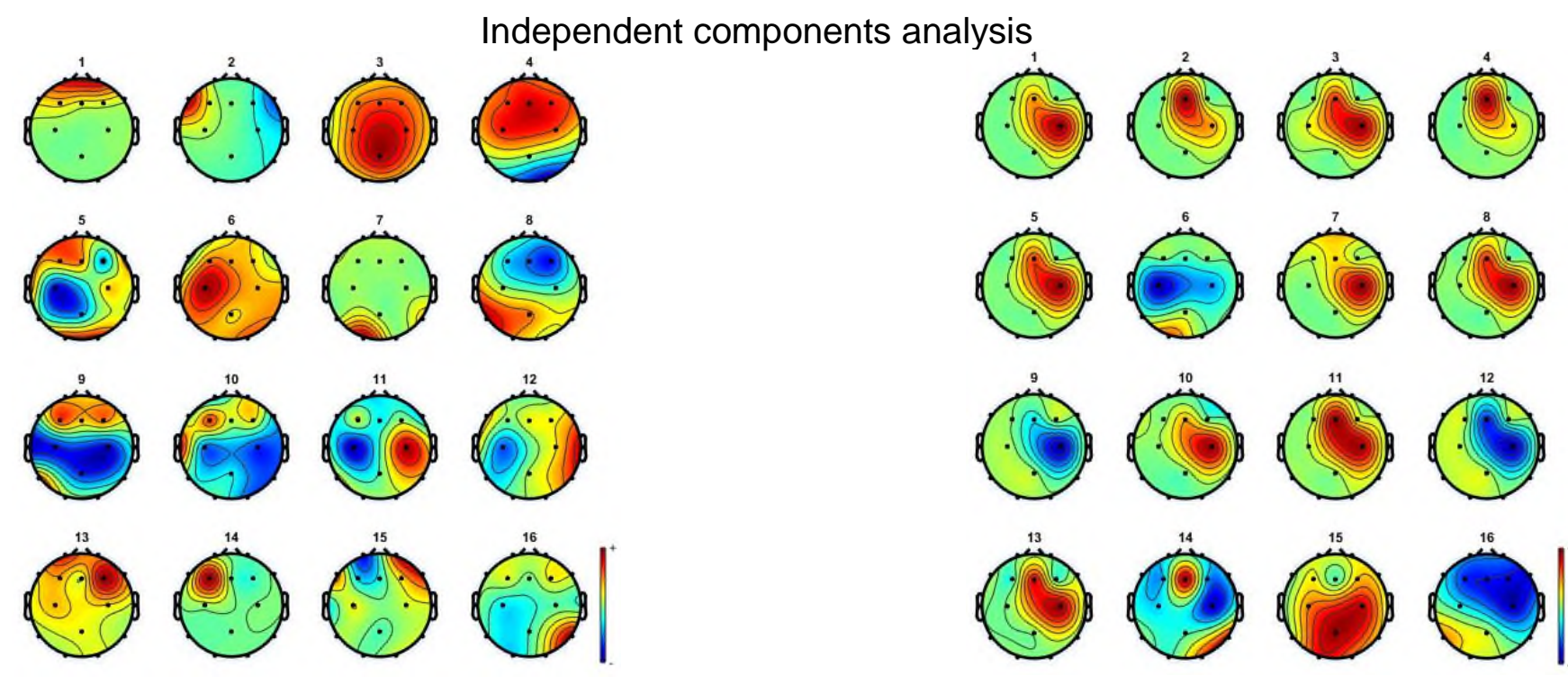

Principal components analysis at $\mathrm{P} 1$
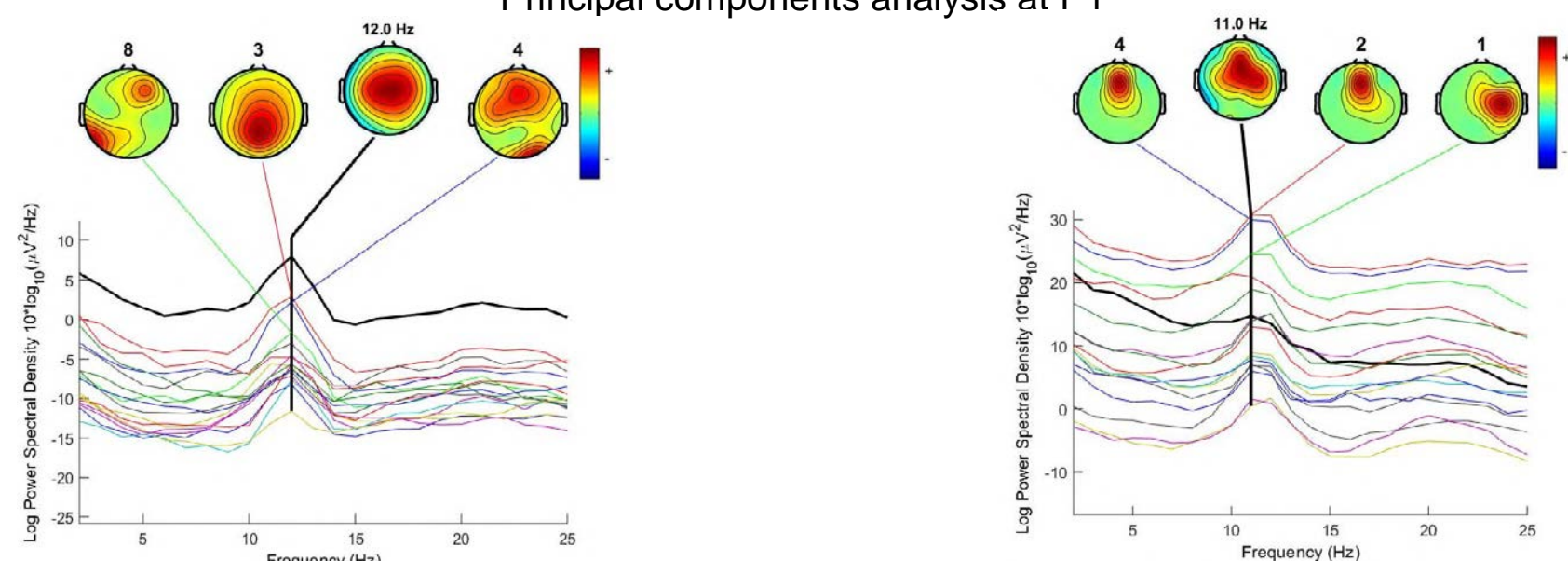

Figure 39: ERPs Principal and Independent Component Analysis for Patient 9 - Binaural 396Hz (Session 1 and 4) 


$\begin{array}{ll}0000 & 000 \\ 0000 & 000 \\ 0000 & 000 \\ 0000 & 000 \\ 0000 & 0901\end{array}$




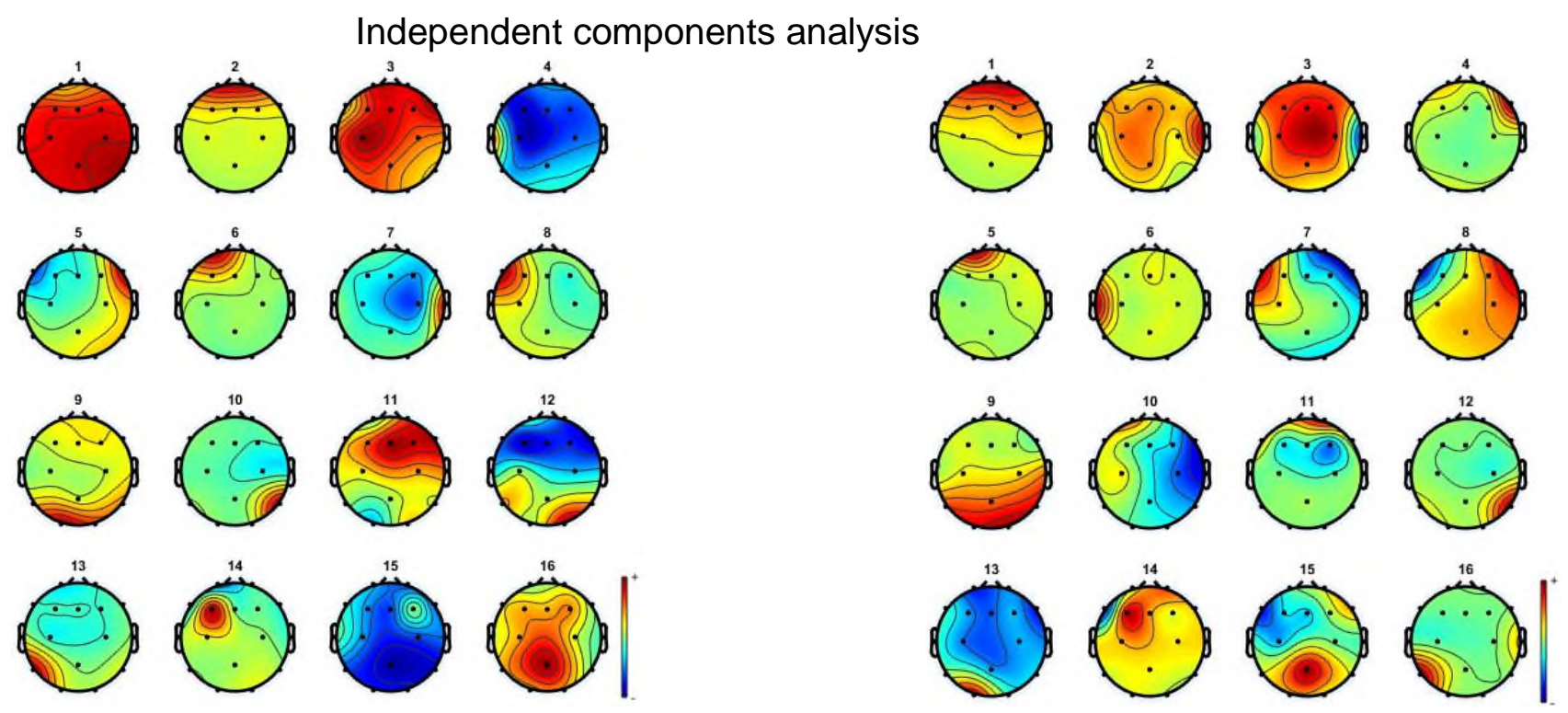

Principal components analysis at $\mathrm{P} 1$
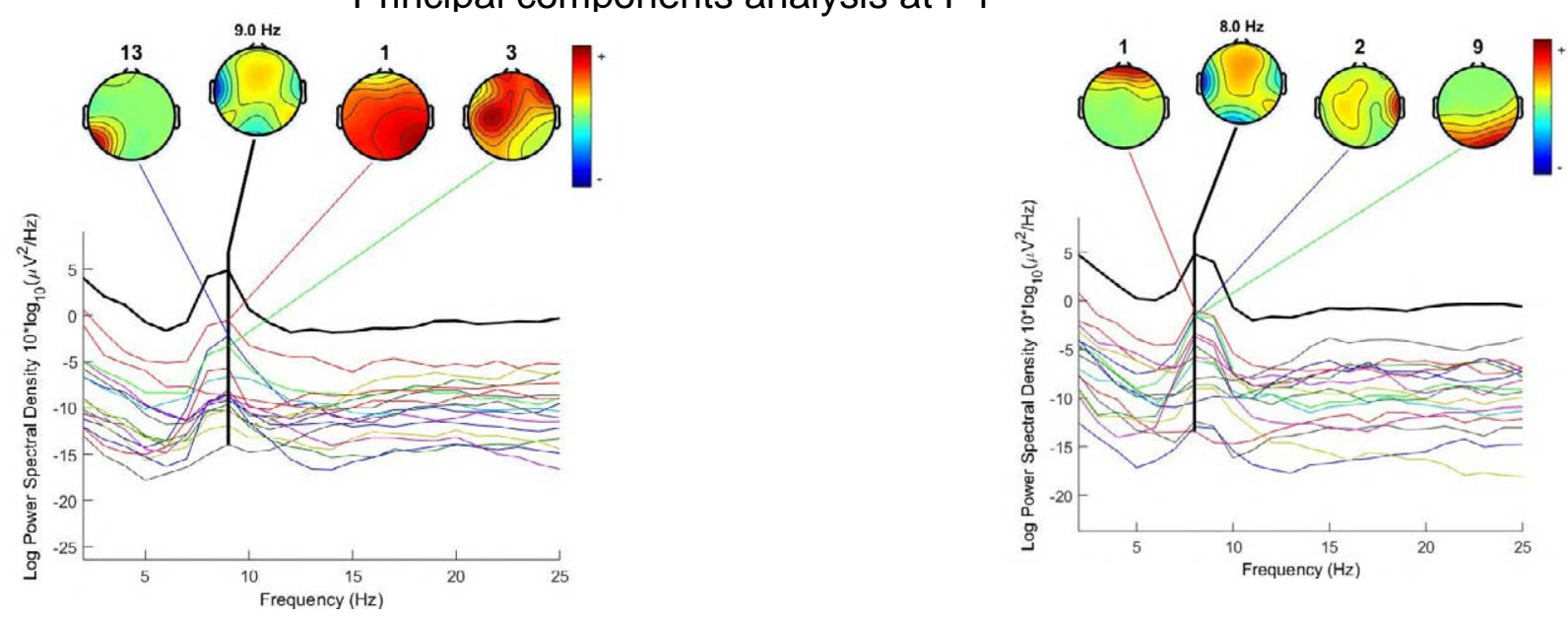

Figure 41: ERPs Principal and Independent Component Analysis for Patient 7 - EAE (Session 1 and 4) 
ANNEX AD: EXAMPLE OF PATIENT PER ACOUSTIC THERAPY FOR ERPS SOURCE LOCATION IN 100MS, 200MS, 300MS AND 400MS.
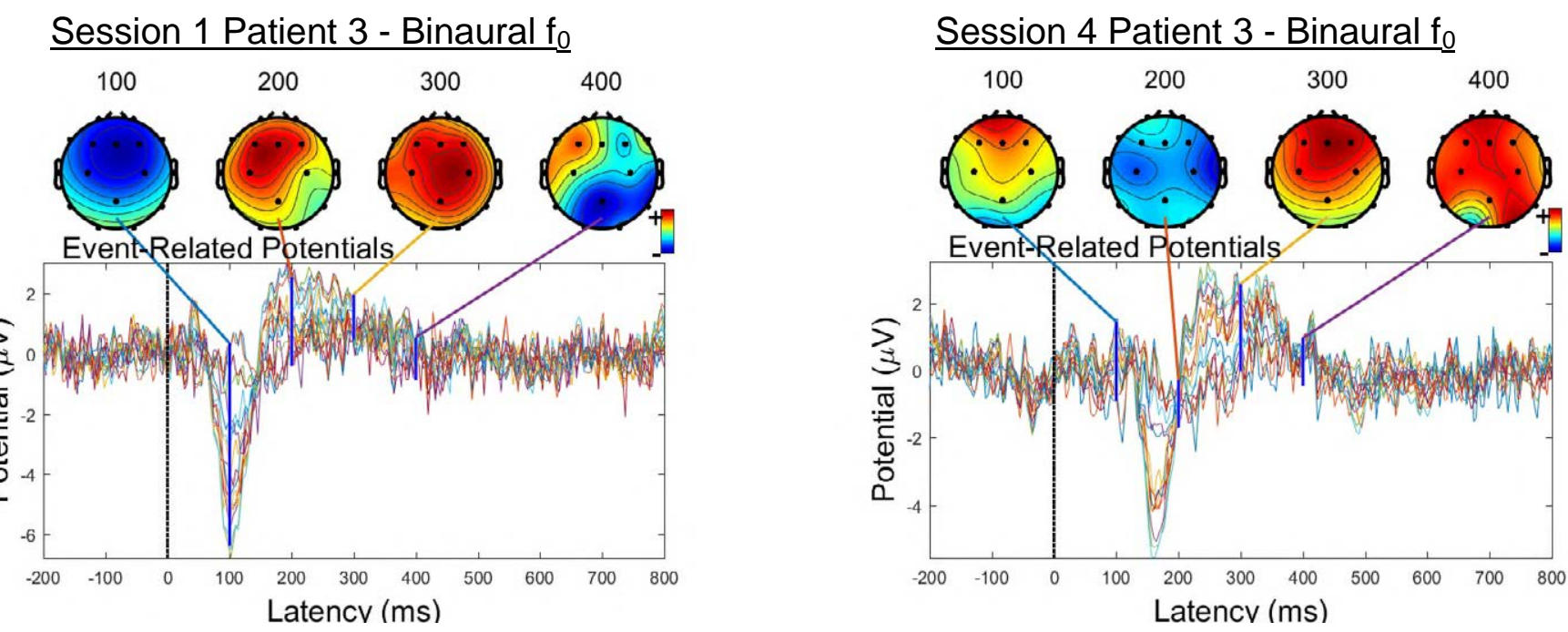

Figure 42: ERPs Source Location (100, 200, 300 \& $400 \mathrm{~ms})$ Patient 3 - Binaural $f_{o}$ (Session 1 and 4) Session 1 Patient 9 - Binaural $396 \mathrm{~Hz}$
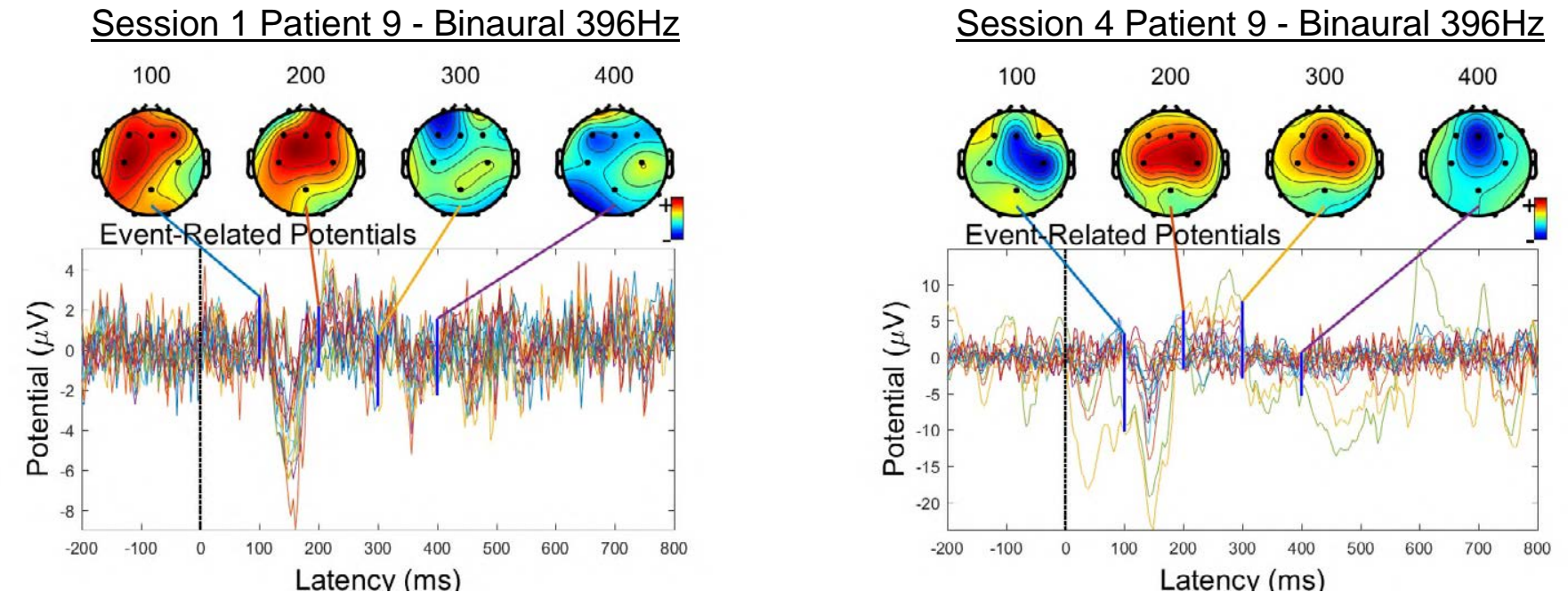

Figure 43: ERPs Source Location (100, 200, 300 \& $400 \mathrm{~ms}$ ) Patient 9 - Binaural 396Hz (Session 1 and 4) 
Session 1 Patient $5-$ TRT

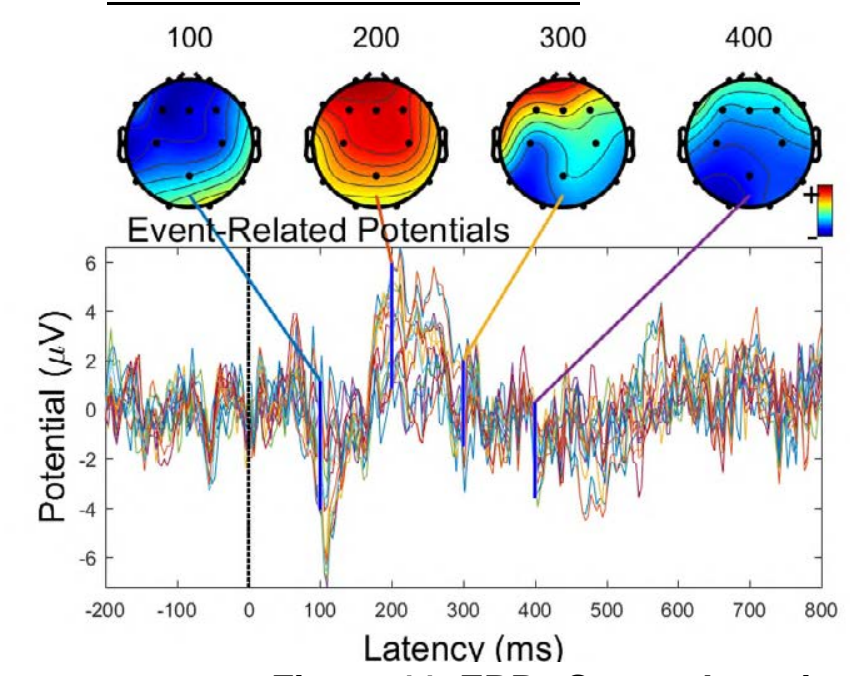

Figure 44: ERPs Source Location (100, 200, 300 \& $400 \mathrm{~ms}$ ) Patient 5 - TRT (Session 1 and 4)
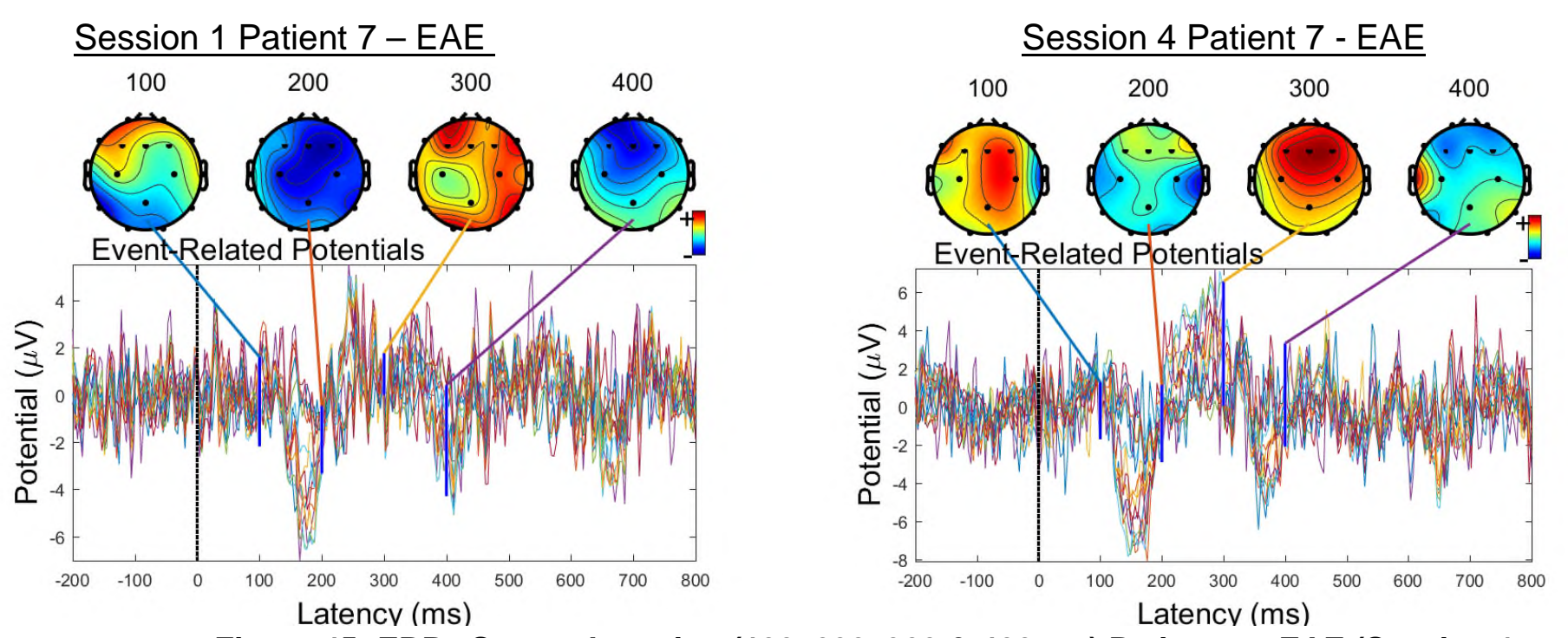

Figure 45: ERPs Source Location (100, 200, 300 \& 400 ms) Patient 7 - EAE (Session 1 and 4) 
ANNEX AE: SESSION 4 - SESSION 1 DIFFERENCE OF TIME AND TREQUENCY DOMAIN PARAMETERS FROM EACH GAV ERP ACOUSTIC THERAPY: SELECTION OF PARAMETERS FOR FRONTAL AND TEMPORAL LOBE

\begin{tabular}{|c|c|c|c|c|c|c|c|c|c|c|c|c|c|c|c|c|c|c|c|c|}
\hline & \multicolumn{10}{|c|}{ Time domain parameters selection } & \multicolumn{10}{|c|}{ Frequency domain parameters selection } \\
\hline $\begin{array}{c}\text { Binaural } \\
\text { fo }\end{array}$ & Mean & P1_ampl & P1_latency & N1_ampl & N1_latency & |P2/3_ampl & | P2/3_latency & STD DEV & RMS & KURTOSIS & PSDtotal & PSD- $\delta$ & PSD- $\theta$ & PSD- $\alpha$ & PSD- $\beta$ & PSD-y & $\mathrm{P} 1(\mathrm{Xmf})$ & P4 & $\mathrm{P} 5(\mathrm{Xfc})$ & $P 13$ \\
\hline 1 & 0.40 & -0.17 & 0.86 & 0.32 & 0.54 & 1.09 & 0.16 & 0.46 & 0.46 & 0.02 & 2.90 & 3.93 & 1.98 & 3.40 & 5.85 & 4.33 & 0.47 & 0.31 & 0.11 & 0.34 \\
\hline 2 & 0.65 & 0.10 & 0.73 & 0.02 & 0.25 & 0.65 & 0.01 & 0.20 & 0.17 & 0.21 & 0.66 & 1.46 & 0.68 & 2.15 & -0.23 & 0.11 & 0.10 & 0.43 & 0.05 & 0.08 \\
\hline 3 & 81.56 & 0.57 & 1.17 & 0.51 & 0.46 & 0.23 & 0.09 & 0.25 & 0.29 & 0.36 & 1.19 & 0.69 & 1.57 & 5.37 & 1.97 & 2.42 & 0.32 & 0.16 & 0.04 & 0.22 \\
\hline 7 & 0.21 & 0.05 & 0.44 & 0.19 & 0.25 & 0.23 & 0.12 & 0.04 & 0.06 & 0.37 & 0.28 & 0.11 & 0.43 & 1.30 & -0.60 & 0.83 & 0.15 & 0.06 & 0.12 & 0.12 \\
\hline $\begin{array}{c}\text { Binaural } \\
396 \mathrm{~Hz}\end{array}$ & Mean & P1_ampl & P1_latency & N1_ampl & N1_latency & P2/3_ampl & | P2/3_latency & STD DEV & RMS & KURTOSIS & PSDtotal & PSD- $\delta$ & PSD- $\theta$ & PSD- $\alpha$ & PSD- $\beta$ & PSD-y & $P 1(X m f)$ & P4 & $\mathrm{PS}(\mathrm{Xfc})$ & P13 \\
\hline 1 & 1.86 & 0.99 & 0.36 & 0.00 & - & 0.27 & 0.18 & 0.02 & 0.01 & 0.08 & 0.12 & 0.34 & 0.04 & 0.12 & -0.22 & 0.06 & 0.05 & 0.13 & 0.01 & 0.03 \\
\hline 2 & 1.57 & 0.28 & 0.04 & 0.15 & 0.03 & 0.13 & 0.21 & 0.10 & 0.10 & 0.30 & 0.17 & 1.41 & 0.09 & 0.42 & 0.32 & 1.18 & 0.12 & 0.09 & 0.02 & 0.09 \\
\hline 3 & 1.18 & 0.58 & 0.04 & 0.14 & 0.11 & 0.20 & 0.12 & 0.10 & 0.13 & 0.18 & 0.59 & 0.58 & 0.27 & 0.31 & -0.22 & 0.14 & 0.09 & 0.15 & 0.13 & 0.06 \\
\hline 7 & 0.92 & 0.19 & 0.08 & 0.14 & 0.05 & 0.02 & 0.19 & 0.03 & 0.03 & 0.17 & 0.00 & 0.61 & 0.44 & 0.64 & -0.78 & 2.77 & 0.09 & 0.15 & 0.13 & 0.09 \\
\hline TRT & Mean & P1_ampl & P1_latency & N1_ampl & N1_latency & P2/3_ampl & P2/3_latency & STD DEV & RMS & KURTOSIS & PSDtotal & PSD- $\delta$ & PSD- $\theta$ & PSD- $\alpha$ & PSD- $\beta$ & PSD- $y$ & $\mathrm{P} 1(\mathrm{Xmf})$ & P4 & $\mathrm{P5}(\mathrm{Xfc})$ & $\mathrm{P} 13$ \\
\hline 1 & 1.65 & $-\quad 0.43$ & 0.04 & 0.90 & 0.08 & 0.14 & 0.14 & 0.26 & 0.25 & 0.38 & 2.34 & 2.43 & 3.93 & 9.60 & -0.79 & 0.89 & 0.07 & 0.05 & $-\quad 0.31$ & 0.05 \\
\hline 2 & 1.44 & $-\quad 0.57$ & 0.09 & 1.01 & 0.14 & 0.08 & - & 0.27 & 0.24 & 0.30 & 1.81 & 2.04 & 4.36 & 26.96 & -0.75 & -0.68 & 0.01 & 0.17 & $-\quad 0.21$ & 0.01 \\
\hline 3 & 2.04 & 0.03 & 0.04 & 0.34 & 0.11 & 0.10 & 0.16 & 0.33 & 0.33 & 0.25 & 2.74 & 2.72 & 3.92 & 17.11 & 0.13 & -0.76 & 0.12 & 0.03 & 0.23 & 0.10 \\
\hline 7 & 2.70 & -0.36 & 0.04 & 0.45 & - & 0.15 & 0.03 & 0.24 & 0.25 & 0.22 & 1.69 & 1.74 & 3.52 & 15.53 & -0.67 & -0.80 & -0.03 & 0.23 & $-\quad 0.27$ & 0.04 \\
\hline EAE & Mean & P1_ampl & P1_latency & N1_ampl & N1_latency & P2/3_ampl & P2/3_latency & STD DEV & RMS & KURTOSIS & PSDtotal & PSD- $\delta$ & PSD- $\theta$ & PSD- $\alpha$ & PSD- $\beta$ & PSD- $\gamma$ & $\mathrm{P} 1(\mathrm{Xmf})$ & P4 & $\mathrm{P5}(\mathrm{Xfc})$ & $\mathrm{P} 13$ \\
\hline 1 & 2.98 & $\begin{array}{r}-\quad 0.33 \\
\end{array}$ & 0.11 & 0.00 & - & 0.08 & 0.11 & 0.02 & 0.02 & 0.01 & 0.25 & 0.27 & 0.12 & 0.11 & -0.33 & -0.82 & $\begin{array}{r}-0.11 \\
\end{array}$ & 0.02 & $-\quad 0.16$ & 0.09 \\
\hline 2 & 2.92 & $-\quad 0.58$ & 0.04 & 0.31 & 0.05 & 0.06 & 0.12 & 0.07 & 0.07 & 0.18 & 0.57 & 0.97 & $-\quad 0.05$ & 0.47 & -0.65 & -0.86 & -0.07 & 0.03 & $-\quad 0.15$ & 0.06 \\
\hline 3 & 0.72 & - $\quad 0.50$ & 0.14 & 0.00 & 0.05 & 0.14 & 0.14 & 0.08 & 0.09 & 0.02 & 0.66 & 0.69 & 0.16 & 0.24 & -0.58 & 0.57 & 0.02 & 0.02 & $\begin{array}{r}-\quad 0.12 \\
\end{array}$ & 0.02 \\
\hline 7 & 10.00 & 0.47 & 0.15 & 0.22 & 0.07 & 0.04 & 0.01 & 0.11 & 0.12 & 0.21 & 0.89 & 1.69 & 0.00 & 0.70 & -0.72 & 0.58 & 0.02 & 0.05 & 0.15 & 0.03 \\
\hline
\end{tabular}

Table 39: Difference between session 4 and session 1: time and frequency parameters selection for GAv analysis 


\section{ANNEX AF: SESSION 4 - SESSION 1 DIFFERENCE OF TIME AND TREQUENCY DOMAIN PARAMETERS FROM EACH GAV ERP ACOUSTIC THERAPY}

In the following tables are found the time and frequency domain parameters difference between session 4 and session 1 (relativized by Session 1) for ERP signals of all patients per acoustic therapy: Binaural $\mathrm{f}_{0}$ and $396 \mathrm{~Hz}$, TRT and EAE.

- Binaural $\mathbf{f}_{0}$ (data from 3 patients)

Frequency domain parameters

\begin{tabular}{|c|c|c|c|c|c|c|c|c|c|c|c|c|c|c|c|c|c|c|c|c|c|c|}
\hline Channel & PSDtotal & PSD- $\delta$ & PSD $-\theta$ & PSD- $\alpha$ & PSD $-\beta$ & PSD- $-x$ & $\mathrm{P} 1(\mathrm{Xmf})$ & P2 & P3 & P4 & P5(Xfc) & P6 & P7(Xrmsf) & P8 & P9 & P10 & P11 & P12 & P13 & P14 & MDF & PSDef \\
\hline 1 & 2.90 & 3.93 & 1.98 & 3.40 & 5.85 & 4.33 & 0.47 & 1.14 & 0.11 & 0.31 & \begin{tabular}{|l|}
0.11 \\
\end{tabular} & 0.29 & $\begin{array}{l}0.09 \\
\end{array}$ & 0.03 & 0.05 & 0.16 & 0.40 & -0.43 & 0.34 & 0.07 & 0.15 & -0.02 \\
\hline 2 & 0.66 & 1.46 & 0.68 & 2.15 & -0.23 & 0.11 & 0.10 & 0.47 & 0.21 & 0.43 & -0.05 & 0.02 & 0.04 & -0.01 & -0.03 & 0.07 & 0.06 & -0.00 & 0.08 & -0.03 & 0.14 & 0.01 \\
\hline 3 & 1.19 & 0.69 & 1.57 & 5.37 & 1.97 & 2.42 & 0.32 & 0.58 & -0.09 & 0.16 & -0.04 & 0.11 & 0.03 & -0.02 & -0.01 & 0.16 & 0.08 & -0.22 & 0.22 & -0.03 & 0.12 & 0.01 \\
\hline 4 & 0.14 & 0.16 & 0.70 & 3.87 & 0.56 & 0.16 & 0.01 & 0.18 & 0.02 & 0.07 & -0.15 & -0.12 & 0.13 & -0.06 & 0.07 & 0.04 & 0.28 & 0.28 & -0.02 & -0.12 & 0.27 & 0.02 \\
\hline 5 & 0.07 & 0.26 & 0.25 & 4.67 & 0.44 & 0.65 & 0.07 & 0.10 & -0.07 & 0.16 & -0.01 & -0.02 & 0.04 & -0.07 & 0.04 & -0.01 & 0.09 & -0.15 & 0.07 & -0.06 & - & -0.02 \\
\hline 6 & 0.12 & 0.11 & 0.09 & 4.34 & 0.59 & 0.42 & 0.08 & 0.20 & -0.02 & 0.02 & -0.01 & 0.06 & 0.01 & 0.02 & -0.02 & 0.07 & 0.01 & -0.06 & 0.07 & 0.02 & 0.07 & 0.01 \\
\hline 7 & 0.28 & 0.11 & 0.43 & 1.30 & -0.60 & 0.83 & 0.15 & 0.02 & 0.09 & 0.06 & -0.12 & -0.04 & 0.05 & 0.07 & -0.11 & 0.09 & 0.86 & 0.45 & -0.12 & 0.04 & 0.48 & 0.08 \\
\hline 8 & 17.22 & 6.73 & 2.29 & 5.21 & 0.15 & 0.49 & 0.22 & 1.80 & 0.36 & 0.76 & -0.23 & 0.06 & 0.14 & -0.00 & 0.14 & 0.38 & 0.42 & 0.13 & 0.15 & -0.04 & 0.50 & 0.12 \\
\hline 9 & 0.10 & 0.21 & 0.45 & 6.84 & 0.68 & 1.37 & 0.17 & 0.15 & 0.10 & 0.19 & 0.06 & 0.10 & 0.04 & -0.01 & 0.04 & 0.03 & 0.17 & -0.24 & 0.13 & 0.01 & 0.08 & -0.02 \\
\hline 10 & 0.20 & 0.43 & 0.33 & 15.37 & 1.75 & 0.02 & 0.06 & 0.07 & -0.09 & 0.16 & -0.07 & -0.04 & 0.07 & -0.06 & -0.01 & 0.03 & 0.02 & -0.03 & 0.05 & -0.07 & - & -0.00 \\
\hline 11 & 0.53 & 0.72 & 0.04 & 7.74 & -0.91 & 0.67 & 0.16 & 0.15 & 0.14 & 0.27 & -0.08 & -0.08 & 0.05 & 0.04 & -0.08 & 0.00 & 0.69 & 0.50 & -0.15 & 0.00 & 0.13 & 0.04 \\
\hline 12 & 0.34 & 0.23 & 0.29 & 0.40 & 4.36 & 2.29 & 0.17 & 0.27 & -0.18 & 0.29 & -0.01 & 0.04 & 0.02 & -0.03 & 0.01 & 0.05 & 0.08 & -0.14 & 0.12 & $\begin{array}{r}-0.04 \\
-0.04\end{array}$ & 0.03 & -0.01 \\
\hline 13 & 0.39 & 0.78 & 0.75 & 49.94 & 0.73 & 0.08 & 0.00 & 0.02 & 0.02 & 0.06 & 0.02 & 0.01 & 0.00 & 0.00 & 0.00 & -0.03 & 0.04 & 0.03 & -0.00 & -0.01 & & 0.02 \\
\hline 14 & 0.07 & 0.63 & 0.67 & 2.00 & $\begin{array}{r}-0.12 \\
-0.0\end{array}$ & 1.00 & 0.10 & 0.03 & 0.07 & 0.32 & 0.04 & 0.01 & 0.01 & $\begin{array}{r}-0.03 \\
-0.03\end{array}$ & 0.04 & $\begin{array}{r}-0.03 \\
-0.03\end{array}$ & 0.15 & $\begin{array}{l}-0.12 \\
-0.12\end{array}$ & 0.06 & $\begin{array}{r}-0.04 \\
-0.04\end{array}$ & 0.22 & $\begin{array}{r}-0.03 \\
-0.03\end{array}$ \\
\hline 15 & 2.96 & 7.95 & 7.16 & 0.54 & 1.92 & 2.14 & 0.33 & 0.69 & -0.27 & 0.53 & 0.01 & 0.20 & 0.02 & 0.03 & -0.01 & 0.19 & 0.17 & -0.24 & 0.25 & 0.04 & 0.04 & 0.01 \\
\hline 16 & 0.78 & 0.96 & 0.95 & 0.67 & 0.13 & 0.34 & 0.13 & 0.43 & -0.06 & 0.03 & 0.14 & -0.14 & 0.05 & -0.03 & 0.09 & -0.24 & 0.36 & 0.15 & -0.12 & -0.08 & 0.48 & -0.07 \\
\hline
\end{tabular}

Table 40: Frequency domain parameters SESSION 4 - SESSION 1 difference for GAv ERP - Binaural $f_{0}$ Therapy

Time domain parameters

\begin{tabular}{|c|c|c|c|c|c|c|c|c|c|c|c|c|c|c|c|c|c|c|c|c|c|c|c|c|}
\hline channel & Mean & MeanAbs & Median & P1_ampl & P1_latency & N1_ampl & N1_latency & P2/3_ampl & P2/3_latency & SSI & IEEG & WL & MMAV1 & MMAV2 & STD DEV & VAR & ROOT & RMS & CREST & SKEWNESS & \begin{tabular}{|l|l|l|} 
& KURTOSIS \\
\end{tabular} & 5 CLEARANCE & SHAPE & E IMPULSE \\
\hline 1 & 0.40 & \begin{tabular}{|l|}
0.42 \\
\end{tabular} & 0.77 & 0.17 & 0.86 & 0.32 & \begin{tabular}{|l|}
0.54 \\
\end{tabular} & 1.09 & \begin{tabular}{|l|}
0.16 \\
\end{tabular} & 1.14 & 0.42 & 0.72 & $\begin{array}{l}0.44 \\
\end{array}$ & 0.46 & \begin{tabular}{|l|}
0.46 \\
\end{tabular} & 1.14 & 0.38 & 0.46 & -0.10 & 0.64 & $\begin{array}{l}0.02 \\
\end{array}$ & 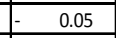 & \begin{tabular}{|l|}
0.03 \\
\end{tabular} & -0.07 \\
\hline 2 & 0.65 & 0.19 & 0.60 & 0.10 & 0.73 & 0.02 & 0.25 & 0.65 & 0.01 & 0.37 & 0.19 & -0.04 & 0.24 & 0.31 & 0.20 & 0.45 & 0.17 & 0.17 & -0.13 & 0.46 & 0.21 & 0.13 & -0.01 & 0.14 \\
\hline 3 & 81.56 & 0.20 & 2.89 & 0.57 & 1.17 & 0.51 & 0.46 & 0.23 & 0.09 & 0.68 & 0.20 & 0.25 & 0.23 & 0.18 & 0.25 & 0.56 & 0.17 & 0.29 & 0.17 & 0.40 & 0.36 & 0.30 & 0.07 & 0.25 \\
\hline 4 & 1.79 & 0.00 & 2.29 & 0.13 & 0.53 & 0.25 & 0.54 & 0.16 & 0.09 & 0.14 & 0.00 & -0.16 & 0.00 & 0.04 & 0.07 & 0.14 & 0.02 & 0.07 & 0.17 & 0.36 & 0.36 & 0.28 & 0.06 & 0.25 \\
\hline 5 & 0.33 & 0.05 & 0.69 & 0.18 & 0.53 & 0.10 & 0.54 & 0.11 & 0.09 & 0.11 & 0.05 & 0.13 & 0.04 & 0.01 & 0.05 & 0.11 & 0.05 & 0.05 & 0.05 & 0.07 & 0.09 & 0.05 & 0.00 & 0.05 \\
\hline 6 & 2.95 & 0.09 & 265.88 & 0.42 & 0.53 & 0.29 & 0.28 & 0.06 & 0.05 & 0.20 & 0.09 & 0.11 & 0.08 & 0.02 & 0.09 & 0.19 & 0.10 & 0.09 & 0.18 & 0.10 & 0.09 & 0.18 & 0.00 & 0.19 \\
\hline 7 & 0.21 & 0.09 & 0.15 & 0.05 & 44 & 0.19 & & 0.23 & & 0.12 & 0.09 & -0.33 & 0.03 & 0.05 & 0.04 & 0.08 & 0.09 & 0.06 & 0.27 & 0.41 & 0.37 & 0.31 & 0.03 & 0.30 \\
\hline 8 & 89.41 & 0.69 & 448.82 & 0.90 & 0.53 & 1.24 & 0.24 & 0.48 & 0.04 & 2.04 & 0.69 & -0.09 & 0.59 & 0.15 & 0.35 & 0.82 & 0.66 & 0.74 & 0.28 & 2.78 & 0.23 & 0.35 & 0.03 & 0.32 \\
\hline 9 & 2.38 & 0.05 & 0.68 & 0.16 & 1.00 & 0.46 & & 0.04 & & 0.19 & 0.05 & 0.27 & 0.02 & 0.06 & 0.09 & & 0.05 & 0.09 & 0.34 & & 0.33 & 0.40 & 0.03 & 0.39 \\
\hline 10 & 1.69 & 0.02 & 8.60 & 0.02 & 0.53 & 0.35 & 0.25 & 0.15 & 0.02 & 0.11 & 0.02 & 0.04 & 0.01 & 0.10 & 0.03 & 0.05 & 0.06 & 0.05 & 0.29 & 0.80 & 0.59 & 0.28 & 0.03 & 0.32 \\
\hline 11 & 0.28 & 0.08 & 0.08 & 1.27 & 0.11 & 0.27 & 0. & 0.38 & 0.16 & 0.22 & 0.08 & -0.26 & 0.06 & 0.01 & 0.09 & 0.17 & 0.06 & 0.12 & -0.17 & 0.41 & 0.17 & 0.22 & -0.04 & 0.20 \\
\hline 12 & 2.02 & 0.1 & 1.6 & 1.15 & 1.2 & 0.19 & & 0.1 & 0.4 & 0.35 & 0.18 & 0.20 & 0.11 & 0.08 & 0.13 & 0.27 & 0.18 & 0.16 & 0.02 & 0.15 & 0.08 & 0.01 & -0.02 & 0.01 \\
\hline 13 & 0.44 & 0.05 & 2.88 & 0.23 & 0.53 & 0.22 & 0.5 & 0.0 & 0.05 & 0.02 & 0.05 & 0.05 & 0.01 & 0.02 & 0.01 & 0.0 & 0.07 & 0.01 & 0.21 & 0.07 & 0.00 & 0.14 & -0.04 & 0.17 \\
\hline 14 & 0.20 & 0.06 & 0.08 & 0.79 & 0. & 0.14 & 0. & 0.0 & 0.07 & 0.05 & 0.06 & 0.14 & 0.01 & 0.09 & 0.05 & 0.11 & 0.06 & 0.03 & 0.11 & 0.54 & 0.03 & 0.07 & $\begin{array}{c}-0.04 \\
-0.03\end{array}$ & 0.08 \\
\hline 15 & 1.74 & 0.36 & 1.05 & 0.11 & 0.13 & 0.54 & 0.17 & 0.26 & 0.07 & 0.83 & 0.36 & 0.32 & 0.34 & 0.19 & 0.26 & 0.59 & 0.39 & 0.35 & 0.14 & 3.34 & 0.01 & 0.11 & -0.00 & 0.14 \\
\hline 16 & 1.28 & 0.20 & 1.18 & 0.22 & 0.21 & 0.62 & 0.42 & 0.47 & 0.11 & 0.39 & 0.20 & 0.00 & 0.23 & 0.23 & 0.19 & 0.34 & 0.21 & 0.22 & -0.23 & 1.04 & 0.26 & 0.24 & -0.02 & 0.24 \\
\hline
\end{tabular}

Table 41: Time domain parameters SESSION 4 - SESSION 1 difference for GAv ERP - Binaural $\mathrm{f}_{0}$ Therapy 
- Binaural $396 \mathrm{~Hz}$ (data from 7 patients)

Frequency domain parameters

\begin{tabular}{|c|c|c|c|c|c|c|c|c|c|c|c|c|c|c|c|c|c|c|c|c|c|c|}
\hline Channel & PSDtotal & PSD- $\delta$ & PSD- $\theta$ & PSD- $\alpha$ & PSD- $\beta$ & PSD- $\gamma$ & P1(Xmf) & P2 & P3 & P4 & $\mathrm{P} 5(\mathrm{Xfc})$ & P6 & P7(Xrmsf) & P8 & P9 & $\mathrm{P} 10$ & P11 & P12 & $\mathrm{P} 13$ & P14 & $\mathrm{MDF}$ & \begin{tabular}{|l|} 
PSDef \\
\end{tabular} \\
\hline 1 & 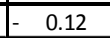 & 0.34 & 0.04 & 0.12 & -0.22 & 0.06 & \begin{tabular}{|c|}
-0.05 \\
\end{tabular} & 0.07 & 0.07 & 0.13 & \begin{tabular}{|l|}
-0.01 \\
\end{tabular} & 0.00 & 0.01 & 0.04 & -0.03 & 0.01 & 0.09 & 0.11 & -0.03 & 0.03 & 0.12 & \begin{tabular}{|l|}
0.02 \\
\end{tabular} \\
\hline 2 & 17 & & 09 & 0.42 & 0.32 & .18 & 0.12 & 0.19 & 0.03 & 0 & 0.02 & 0.06 & 0.01 & 0.01 & 0.00 & 0.04 & 0.06 & -0.07 & 0.09 & 0.00 & 0.07 & -0.01 \\
\hline 3 & & & & 0. & -0.22 & 0.14 & 0.09 & 0.23 & 06 & & 0.13 & 0 & & 0.03 & .06 & -0.1 & 0.12 & -0.05 & -0.06 & .05 & 31 & 0.04 \\
\hline 4 & & 1 & & 0.0 & -0.47 & 0.06 & & 0.23 & 0.06 & 0.12 & -0.05 & 0.02 & 0.02 & 0.04 & -0.06 & \begin{tabular}{|l|}
0.07 \\
\end{tabular} & 0.10 & 0.14 & 0.01 & -0.01 & 0.09 & 0.03 \\
\hline 5 & & & & & 1.18 & 04 & & & 0.19 & & \begin{tabular}{|l|}
-0.12 \\
\end{tabular} & 0.08 & & 0.02 & -0.10 & 0.2 & & 0.6 & 0.18 & -0. & 0.20 & 0.05 \\
\hline 6 & & & & 0. & -0.65 & 0.61 & & 0.07 & 0. & & \begin{tabular}{|l|}
0.11 \\
\end{tabular} & 0.16 & & 0.09 & \begin{tabular}{|l|}
0.03 \\
\end{tabular} & & 10 & -0.15 & 0.05 & 0.1 & & 0.02 \\
\hline 7 & & & & 0.6 & -0.78 & 2.77 & & & 0.05 & & 0.13 & 0.16 & & -0.02 & 0.11 & -0.0 & 0.31 & -0.34 & 0.09 & 0.0 & 0.13 & -0.03 \\
\hline 8 & & & & 0.1 & 0.11 & 0.20 & & 0.79 & 0.15 & 11 & -0.19 & -0.04 & 0.1 & -0.06 & -0.09 & 0.1 & 0.56 & 0.12 & 0.04 & -0.09 & 0.27 & 0.06 \\
\hline 9 & & & & & 0.04 & 0.25 & & 0.79 & 0.06 & & -0.15 & 0.03 & & 0.07 & -0.15 & 0.2 & 0.27 & 0.32 & 0.07 & -0.04 & 0.17 & \begin{tabular}{|l|}
0.07 \\
\end{tabular} \\
\hline 10 & & 17.03 & & 1. & -0.12 & 1.62 & & 1.59 & 0.11 & & -0.05 & 0.31 & 0 & 0.13 & -0.09 & 0.3 & 0.07 & -0.19 & 0.34 & 0.0 & 0.18 & \begin{tabular}{|l|}
0.08 \\
\end{tabular} \\
\hline 11 & & 0.07 & 4.19 & 0.68 & 0.39 & 22.62 & 0.41 & 0.45 & -0.07 & 0.02 & 0.20 & $\begin{array}{ll}0.17 \\
\end{array}$ & 0.10 & -0.04 & $\begin{array}{ll}0.15 \\
\end{array}$ & -0.03 & 0.53 & -0.45 & 0.28 & -0.02 & 0.65 & \begin{tabular}{|l|}
-0.08 \\
\end{tabular} \\
\hline 12 & 679.33 & $1,508.68$ & 214.25 & 29.68 & 3.99 & 1.73 & & 19.46 & 0.49 & & -0.41 & 0.43 & 0.26 & 0.06 & -0.30 & 1.4 & 0.58 & -0.10 & 0.88 & -0.10 & 0.67 & \begin{tabular}{|l|}
0.25 \\
\end{tabular} \\
\hline 13 & & & & & 0.02 & & & 0.25 & 0.01 & & \begin{tabular}{|l|}
-0.10 \\
\end{tabular} & -0.05 & & -0.04 & -0.05 & & & 0.10 & & & & \begin{tabular}{|l|}
0.01 \\
\end{tabular} \\
\hline 14 & 0.77 & 0.93 & 0.64 & 0.81 & 0.54 & 0.79 & 0.07 & 0.41 & -0.24 & 0.40 & \begin{tabular}{|l|} 
\\
\end{tabular} & -0.01 & 0.13 & 0.01 & \begin{tabular}{|l|l|}
0.12 \\
\end{tabular} & -0.18 & 0.25 & -0.14 & -0.04 & 0.03 & 0.35 & -0.07 \\
\hline 15 & 0.64 & 0.91 & 1.86 & 0.07 & 2.29 & 0.26 & 0.06 & 0.29 & 0.06 & 0.16 & -0.09 & 0.04 & 0.06 & 0.00 & -0.06 & 0.14 & 0.43 & 0.00 & 0.06 & 0.01 & 0.17 & \begin{tabular}{|l|}
0.04 \\
\end{tabular} \\
\hline 16 & 0.34 & 0.82 & 0.15 & 0.70 & 0.36 & 2.11 & 0.14 & 0.07 & -0.29 & 0.53 & 0.19 & 0.17 & 0.15 & 0.07 & 0.07 & -0.02 & 0.50 & -0.27 & 0.12 & 0.09 & 0.31 & \begin{tabular}{|c|}
-0.03 \\
\end{tabular} \\
\hline
\end{tabular}

Table 42: Frequency domain parameters SESSION 4 - SESSION 1 difference for GAv ERP - Binaural 396Hz Therapy

$\underline{\text { Time domain parameters }}$

\begin{tabular}{|c|c|c|c|c|c|c|c|c|c|c|c|c|c|c|c|c|c|c|c|c|c|c|c|c|}
\hline Channel & Mean & MeanAbs & Median & P1_ampl & P1_latency & N1_ampl & | N1_latency & P2/3_ampl & P2/3_latency & $\mathrm{SSI}$ & IEEG & WL & MMAV1 & \begin{tabular}{|l|} 
MMAV2 \\
\end{tabular} & STD DEV & VAR & ROOT & RMS & CREST & SKEWNESS & KURTOSIS & CLEARANCE & SHAPE & IMPULSE \\
\hline 1 & 1.86 & \begin{tabular}{|l|}
0.01 \\
\end{tabular} & 2.36 & 0.99 & \begin{tabular}{|c|}
-0.36 \\
\end{tabular} & 0.00 & $=-$ & \begin{tabular}{|c|}
-0.27 \\
\end{tabular} & \begin{tabular}{|c|}
0.18 \\
\end{tabular} & 0.03 & 0.01 & -0.04 & $\begin{array}{ll}0.01 \\
\end{array}$ & \begin{tabular}{|l|}
-0.00 \\
\end{tabular} & \begin{tabular}{|l|}
0.02 \\
\end{tabular} & 0.03 & 0.01 & 0.01 & -0.02 & 0.75 & 0.08 & \begin{tabular}{|l|}
0.00 \\
\end{tabular} & \begin{tabular}{|l|l|}
0.00 \\
\end{tabular} & \begin{tabular}{|l|}
-0.02 \\
\end{tabular} \\
\hline 2 & 1.57 & 0.02 & 1.36 & 0.28 & 0.04 & 0.15 & 0.03 & 0.13 & 0.21 & 0.20 & 0.02 & 0.07 & 0.05 & 0.11 & 0.10 & 0.21 & 0.03 & 0.10 & 0.05 & 0.99 & 0.30 & 0.19 & 0.07 & 0.13 \\
\hline 3 & 1.18 & 0.06 & 1.83 & 0.58 & 0.04 & 0.14 & 0.11 & 0.20 & 0.12 & 0.25 & 0.06 & 0.05 & 0.08 & 0.16 & 0.10 & 0.19 & 0.03 & 0.13 & -0.01 & 0.38 & 0.18 & 0.11 & -0.08 & 0.09 \\
\hline 4 & 2.21 & 0.16 & 1.14 & 0.09 & 0.32 & 0.04 & 0.08 & 0.10 & 0.14 & 0.20 & 0.16 & -0.08 & 0.18 & 0.18 & 0.10 & 0.20 & 0.20 & 0.10 & -0.05 & 0.31 & 0.06 & 0.13 & -0.05 & 0.10 \\
\hline 5 & 25.62 & 0.58 & 1.53 & 0.35 & 0.32 & 0.28 & 0.06 & 0.01 & 0.25 & 0.92 & 0.58 & 0.12 & 0.55 & 0.45 & 0.37 & 0.87 & 0.70 & 0.38 & -0.08 & 0.60 & 0.22 & 0.25 & -0.12 & 0.19 \\
\hline 6 & 1.82 & 0.03 & 1.01 & 0.12 & 0.04 & 0.03 & 0.03 & 0.15 & 0.16 & 0.07 & 0.03 & 0.04 & 0.04 & 0.06 & 0.03 & 0.07 & 0.02 & 0.03 & 0.00 & 0.86 & 0.08 & 0.02 & 0.00 & 0.00 \\
\hline 7 & 0.92 & 0.00 & 1.16 & 0.19 & 0.08 & 0.14 & 0.05 & 0.02 & 0.19 & 0.07 & 0.00 & 0.33 & 0.04 & 0.07 & 0.03 & 0.05 & 0.02 & 0.03 & 0.11 & 0.53 & 0.17 & 0.16 & 0.03 & 0.14 \\
\hline 8 & 2.25 & 0.25 & 0.88 & 0.52 & 0.12 & 0.27 & 0.03 & 0.03 & 0.03 & 0.58 & 0.25 & -0.12 & 0.28 & 0.39 & 0.26 & 0.58 & 0.24 & 0.26 & 0.01 & 0.08 & 0.07 & 0.02 & 0.00 & 0.01 \\
\hline 9 & 2.70 & 0.31 & 1.96 & 0.36 & 0.04 & 0.43 & 0.03 & 0.00 & 0.18 & 0.72 & 0.31 & -0.08 & 0.32 & 0.34 & 0.31 & 0.71 & 0.30 & 0.31 & 0.10 & 0.12 & 0.03 & 0.10 & 0.00 & 0.10 \\
\hline 10 & 10.10 & 0.79 & 8.58 & 1.14 & 0.08 & 0.65 & 0.06 & 0.02 & 0.23 & 1.64 & 0.79 & 0.23 & 0.73 & 0.76 & 0.56 & 1.43 & 0.89 & 0.63 & 0.02 & 0.07 & 0.17 & 0.13 & -0.09 & 0.08 \\
\hline 11 & 0.20 & 0.32 & 3.90 & 0.52 & 0.08 & 0.26 & - & 0.63 & 0.07 & 0.60 & 0.32 & 0.80 & 0.26 & 0.20 & 0.26 & 0.60 & 0.38 & 0.26 & 0.00 & 0.56 & 0.05 & 0.08 & -0.04 & 0.04 \\
\hline 12 & 4.09 & 2.70 & 1.56 & 2.46 & 0.35 & 7.97 & 0.03 & 0.91 & 0.14 & 14.51 & 2.77 & 0.27 & 2.92 & 2.95 & 3.05 & 15.39 & 2.58 & 2.94 & \begin{tabular}{|l}
0.07 \\
\end{tabular} & 4.94 & 0.12 & 0.18 & 0.06 & 0.14 \\
\hline 13 & 1.69 & 0.03 & 1.13 & 0.71 & 0.12 & 0.49 & 0.03 & 0.15 & 0.16 & 0.23 & 0.03 & -0.04 & 0.03 & 0.02 & 0.11 & 0.24 & 0.02 & 0.11 & 0.34 & 2.82 & 0.62 & 0.46 & 0.07 & 0.44 \\
\hline 14 & 1.08 & 0.23 & 0.91 & 0.10 & 0.07 & 0.24 & 0.34 & 0.42 & 0.44 & 0.36 & 0.23 & 0.17 & 0.25 & 0.23 & 0.18 & 0.32 & 0.26 & 0.20 & -0.11 & 1.20 & 0.03 & 0.04 & 0.04 & 0.07 \\
\hline 15 & 2.35 & 0.12 & 1.62 & 0.76 & 0.12 & 0.75 & 0.05 & 0.27 & 0.08 & 0.23 & 0.12 & -0.06 & 0.15 & 0.02 & 0.10 & 0.21 & 0.14 & 0.11 & -0.12 & 0.87 & 0.11 & 0.14 & -0.01 & 0.13 \\
\hline 16 & 0.85 & 0.09 & 3.62 & 0.60 & 0.32 & 0.51 & 0.12 & 0.14 & 0.40 & 0.07 & 0.09 & 0.31 & 0.06 & 0.00 & 0.03 & 0.07 & 0.11 & 0.03 & -0.19 & 1.94 & 0.21 & 0.25 & -0.05 & 0.23 \\
\hline
\end{tabular}

Table 43: Time domain parameters SESSION 4 - SESSION 1 difference for GAv ERP - Binaural 396Hz Therapy 
- $\quad$ TRT (data from 14 patients)

Frequency domain parameters

\begin{tabular}{|c|c|c|c|c|c|c|c|c|c|c|c|c|c|c|c|c|c|c|c|c|c|c|}
\hline Channel & PSDtotal & PSD- $\delta$ & PSD- $\theta$ & PSD- $\alpha$ & PSD- $\beta$ & PSD- $-\gamma$ & $\mathrm{P} 1(\mathrm{Xmf})$ & P2 & P3 & P4 & $P 5(X f c)$ & P6 & $\mathrm{P7}(\mathrm{Xrmsf})$ & P8 & P9 & P10 & P11 & P12 & $\mathrm{P} 13$ & P14 & MDF & PSDef \\
\hline 1 & 2.34 & 2.43 & 3.93 & 9.60 & -0.79 & 0.89 & -0.07 & 0.83 & 0.09 & 0.05 & -0.31 & -0.13 & 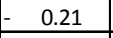 & -0.02 & -0.19 & 0.26 & 0.91 & 0.70 & -0.05 & -0.10 & 0.62 & 0.15 \\
\hline 2 & 1.81 & 2.04 & 4.36 & 26.96 & -0.75 & 0.68 & 0.01 & 0.78 & 0.13 & 0.17 & -0.21 & -0.04 & 0.13 & 0.02 & -0.15 & 0.22 & 0.67 & 0.46 & 0.01 & -0.04 & 0.56 & 0.11 \\
\hline 3 & 2.74 & 2.72 & 3.92 & 17.11 & 0.13 & 0.76 & 0.12 & 0.93 & 0.03 & 0.03 & -0.23 & 0.00 & 0.14 & -0.01 & -0.13 & 0.30 & 0.47 & 0.19 & 0.10 & -0.05 & 0.56 & 0.11 \\
\hline 4 & 1.22 & 1.16 & 1.95 & 33.00 & 0.68 & 0.89 & 0.00 & 0.60 & -0.07 & 0.16 & -0.32 & 0.11 & 0.20 & 0.05 & -0.24 & 0.30 & 0.77 & 0.93 & 0.05 & 0.11 & 0.43 & 0.17 \\
\hline 5 & 1.16 & 1.25 & 1.65 & 24.52 & -0.61 & 0.90 & 0.00 & 0.58 & -0.04 & 0.11 & -0.31 & 0.12 & 0.20 & 0.06 & -0.25 & 0.28 & 0.72 & 1.01 & -0.06 & -0.12 & 0.20 & 0.16 \\
\hline 6 & 1.32 & 1.78 & 1.51 & 31.94 & -0.59 & 0.94 & 0.00 & 0.65 & -0.01 & 0.05 & -0.34 & -0.14 & 0.22 & 0.06 & -0.27 & 0.30 & 0.88 & 1.19 & 0.08 & -0.14 & 0.33 & 0.18 \\
\hline 7 & 1.69 & 1.74 & 3.52 & 15.53 & -0.67 & 0.80 & 0.03 & 0.73 & 0.13 & 0.23 & -0.27 & -0.09 & 0.17 & 0.02 & -0.19 & 0.25 & 0.91 & 0.76 & 0.04 & 0.08 & 0.61 & 0.14 \\
\hline 8 & 1.33 & 1.03 & 2.75 & 8.20 & -0.08 & 0.77 & 0.07 & 0.35 & 0.18 & 0.28 & -0.18 & -0.05 & 0.11 & 0.02 & -0.13 & 0.17 & 0.86 & 0.41 & 0.06 & -0.01 & 0.41 & 0.09 \\
\hline 9 & 1.11 & 1.30 & 1.80 & 16.56 & 0.40 & 0.87 & 0.04 & 0.59 & 0.05 & 0.14 & -0.26 & -0.07 & 0.16 & 0.03 & -0.19 & 0.25 & 0.57 & 0.62 & -0.02 & -0.09 & 0.38 & 0.13 \\
\hline 10 & 0.29 & 0.32 & 0.15 & 6.93 & -0.87 & 0.92 & 0.20 & 0.04 & -0.00 & 0.03 & -0.26 & 0.22 & 0.18 & -0.04 & -0.15 & 0.04 & 0.53 & 0.81 & 0.18 & -0.13 & 0.20 & 0.10 \\
\hline 11 & & & & 3.60 & -0.68 & 0.70 & 0.05 & 0.41 & 0.24 & 0.37 & -0.12 & 0.00 & 0.05 & 0.05 & -0.10 & 0.14 & 0.46 & 0.26 & 0.03 & 0.03 & 0.36 & 0.08 \\
\hline 12 & 0.42 & 0.78 & 0.52 & 0.22 & -0.81 & 0.95 & 0.35 & 0.38 & 0.34 & 0.64 & -0.15 & -0.15 & 0.08 & 0.04 & -0.11 & 0.00 & 1.35 & 0.72 & 0.27 & 0.05 & 0.31 & 0.09 \\
\hline 13 & 0.18 & 0.48 & 2.13 & 0.37 & -0.63 & 0.96 & 0.29 & 0.11 & 0.06 & 0.02 & -0.25 & 0.21 & 0.16 & 0.02 & -0.17 & 0.06 & 1.11 & 1.11 & 0.25 & -0.06 & 0.58 & 0.13 \\
\hline 14 & & 0.67 & 0.25 & 1.61 & -0.80 & 0.83 & 0.19 & 0.01 & 0.48 & 1.29 & -0.09 & 0.09 & 0.05 & 0.01 & -0.05 & 0.01 & 0.36 & 0.34 & 0.12 & 0.01 & 0.32 & 0.05 \\
\hline 15 & 33.30 & 120.52 & 5.80 & 0.67 & 0.92 & 2.36 & 0.38 & 3.71 & 1.61 & 6.10 & -0.15 & 0.20 & 0.09 & -0.01 & -0.07 & 0.42 & 0.34 & 0.20 & 0.33 & 0.02 & 0.28 & 0.08 \\
\hline 16 & 81.58 & 154.44 & 5.76 & 4.95 & -0.40 & 0.52 & 0.23 & 3.49 & 1.67 & 5.85 & -0.24 & 0.15 & 0.14 & -0.03 & -0.11 & 0.51 & 0.53 & -0.20 & 0.23 & 0.04 & 0.46 & 0.13 \\
\hline
\end{tabular}

Table 44: Frequency domain parameters SESSION 4 - SESSION 1 difference for GAv ERP - TRT Therapy

Time domain parameters

\begin{tabular}{|c|c|c|c|c|c|c|c|c|c|c|c|c|c|c|c|c|c|c|c|c|c|c|c|c|}
\hline Channel & Mean & MeanAbs & Median & P1_ampl & P1_latency & N1_ampl & N1_latency & P2/3_ampl & P2/3_latency & SSI & IEEG & WL & MMAV1 & MMAV2 & STD DEV & VAR & ROOT & RMS & CREST & SKEWNESS & S KURTOSIS & CLEARANCE & E SHAPE & IMPULSE \\
\hline 1 & 1.65 & $\begin{array}{l}0.08 \\
\end{array}$ & 26.25 & \begin{tabular}{|l|}
-0.43 \\
\end{tabular} & 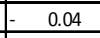 & 0.90 & \begin{tabular}{|l|} 
\\
\end{tabular} & \begin{tabular}{|l|}
-0.14 \\
\end{tabular} & $\begin{array}{ll} & 0.14 \\
\end{array}$ & 0.57 & 0.08 & -0.31 & 0.16 & 0.30 & \begin{tabular}{|l|l|}
0.26 \\
\end{tabular} & 0.58 & 0.02 & 0.25 & \begin{tabular}{ll|}
0.00 \\
\end{tabular} & \begin{tabular}{|l|}
-1.74 \\
\end{tabular} & $\begin{array}{l} \\
\end{array}$ & 0.29 & 0.16 & 0.16 \\
\hline 2 & 1.44 & 0.15 & 2.07 & 0.57 & 0.09 & 1.01 & 0.14 & 0.08 & & 0.54 & 0.15 & -0.21 & 0.23 & 0.43 & 0.27 & 0.62 & 0.09 & 0.24 & 0.01 & 1.59 & 0.30 & 0.15 & 0.08 & 0.08 \\
\hline 3 & 2.04 & 0.23 & 4.30 & 0.03 & 0.04 & 0.34 & 0.11 & 0.10 & 0.16 & 0.78 & 0.23 & -0.14 & 0.26 & 0.30 & 0.33 & 0.78 & 0.17 & 0.33 & 0.01 & 3.41 & 0.25 & 0.14 & 0.09 & 0.09 \\
\hline 4 & 8.02 & & & 0.28 & 0.04 & & & & & 0.50 & 0.15 & -0.33 & & 0.21 & 0.22 & & & & -0.02 & & & 0.07 & 0.07 & 0.04 \\
\hline 5 & 3.61 & 0.19 & 6.00 & 0.67 & & 0.24 & 0.03 & 0.01 & 0.05 & 0.51 & 0.19 & -0.32 & 0.21 & 0.24 & 0.22 & 0.48 & 0.15 & 0.23 & 0.01 & 1.28 & 0.05 & 0.08 & 0.03 & 0.05 \\
\hline 6 & 4.30 & 0.26 & 4.61 & 0.52 & 0.04 & 0.20 & 0.14 & 0.07 & 0.12 & 0.55 & 0.26 & -0.38 & 0.29 & 0.33 & 0.23 & 0.52 & 0.27 & 0.25 & -0.04 & 1.67 & 0.01 & 0.06 & -0.01 & 0.04 \\
\hline 7 & 2.70 & & & 0.36 & & & & & & & 0.18 & -0.30 & & & & & & & 0.07 & & & & 0.06 & 0.13 \\
\hline 8 & 2.43 & 0.04 & 3.45 & 0.37 & & 0.04 & 0.25 & 0.18 & 0.04 & 0.20 & 0.04 & -0.30 & 0.10 & 0.16 & 0.09 & 0.19 & 0.01 & 0.09 & -0.09 & 2.07 & 0.15 & 0.01 & 0.05 & 0.04 \\
\hline 9 & 2.74 & & & 0.29 & 0.22 & 0.20 & & 04 & 0.14 & 0.51 & 0.20 & -0.25 & 0.24 & 0.31 & 0.22 & 0.50 & 0.18 & 0.23 & -0.04 & 1.63 & 0.01 & 0.00 & 0.02 & 0.02 \\
\hline 10 & 0.28 & & & 3.15 & & 0. & & & 0.14 & 0.10 & 0.16 & -0.35 & & 0.11 & 0.0 & 0.08 & 0. & 0.05 & -0.06 & 3.05 & 0.14 & 0.16 & 0.12 & 0.05 \\
\hline 11 & 3.52 & 0.05 & 2.42 & 0.16 & 0.22 & 0.31 & 0.13 & 0.06 & 0.13 & 0.28 & 0.05 & -0.21 & 0.08 & 0.08 & 0.10 & 0.20 & 0.02 & 0.13 & \begin{tabular}{|l}
0.15 \\
\end{tabular} & 0.58 & 0.41 & 0.28 & 0.08 & 0.24 \\
\hline 12 & 1.96 & 0. & 1. & 0.69 & & 0.27 & & 0.67 & 0.1 & 0.44 & 0.24 & -0.45 & 0.2 & 0.14 & 0.28 & 0.48 & 0.24 & 0.25 & -0.02 & 20.62 & 0.20 & 0.04 & -0.01 & 0.03 \\
\hline 13 & 10.50 & 0.21 & 0.95 & 0.67 & 0.22 & 0.03 & 0.22 & 0.06 & 0.01 & 0.19 & 0.21 & -0.44 & 0.16 & 0.07 & 0.12 & 0.22 & 0.27 & 0.10 & \begin{tabular}{|l}
0.05 \\
\end{tabular} & 0.43 & 0.58 & 0.29 & 0.14 & 0.20 \\
\hline 14 & 1.96 & 0.11 & 1.77 & 0.84 & 0.15 & 1.100 & 0.16 & 0.64 & 0.11 & 0.10 & 0.11 & -0.26 & 0.07 & 0.06 & 0.05 & 0.10 & 0.15 & 0.05 & \begin{tabular}{|l|l}
0.27 \\
\end{tabular} & 29.33 & 0.34 & 0.42 & 0.07 & 0.36 \\
\hline 15 & 0.41 & 0.82 & 0.18 & 0.96 & 0.17 & 1.84 & 0.95 & 0.41 & 0.1 & 2.44 & 0.82 & 0.28 & 0.78 & 0.47 & 0.94 & 2.76 & 0.81 & 0.86 & \begin{tabular}{|l}
0.10 \\
\end{tabular} & 2.49 & 0.21 & 0.13 & 0.02 & 0.11 \\
\hline 16 & 4.57 & 0.91 & 3.80 & 0.78 & 0.06 & 0.68 & 1.19 & 0.68 & 0.13 & 2.66 & 0.91 & -0.01 & 1.08 & 1.22 & 0.67 & 1.78 & 0.88 & 0.91 & -0.12 & 5.37 & 0.21 & 0.11 & 0.00 & 0.12 \\
\hline
\end{tabular}

Table 45: Time domain parameters SESSION 4 - SESSION 1 difference for GAv ERP - TRT Therapy 
- $\mathbf{E A E}$ (data from 10 patients)

Frequency domain parameters

\begin{tabular}{|c|c|c|c|c|c|c|c|c|c|c|c|c|c|c|c|c|c|c|c|c|c|c|}
\hline Channel & PSDtotal & PSD- $\delta$ & PSD- $\theta$ & PSD- $\alpha$ & PSD- $\beta$ & PSD- $-\gamma$ & $\mathrm{P} 1(\mathrm{Xmf})$ & P2 & P3 & P4 & $\mathrm{P5}(\mathrm{Xfc})$ & P6 & P7(Xrmsf) & P8 & P9 & $\mathrm{P} 10$ & P11 & P12 & P13 & P14 & MDF & PSDef \\
\hline 1 & 0.25 & 0.27 & 0.12 & 0.11 & -0.33 & 0.82 & 0.11 & 0.08 & 0.01 & 0.02 & -0.16 & -0.10 & 0.10 & -0.01 & -0.08 & 0.07 & 0.26 & 0.30 & 0.09 & -0.05 & 0.23 & 0.07 \\
\hline 2 & 0.57 & 0.97 & 0.05 & 0.47 & -0.65 & 0.86 & 0.07 & 0.20 & 0.01 & 0.03 & -0.15 & -0.03 & 0.06 & 0.06 & -0.12 & 0.15 & 0.33 & 0.37 & 0.06 & 0.01 & 0.31 & 0.11 \\
\hline 3 & 0.66 & 0.69 & 0.16 & 0.24 & -0.58 & 0.57 & 0.02 & 0.21 & 0.01 & 0.02 & -0.12 & -0.04 & 0.06 & -0.00 & -0.06 & 0.09 & 0.15 & 0.17 & -0.02 & -0.02 & 0.23 & 0.06 \\
\hline 4 & 0.34 & 0.52 & 0.05 & 0.17 & -0.76 & 0.80 & 0.10 & 0.11 & 0.01 & 0.03 & -0.21 & -0.12 & 0.13 & 0.04 & -0.16 & 0.12 & 0.42 & 0.69 & 0.10 & 0.07 & 0.20 & 0.11 \\
\hline 5 & 0.30 & 0.67 & 0.06 & 0.09 & -0.68 & 0.77 & 0.05 & 0.11 & -0.01 & 0.01 & -0.13 & 0.02 & 0.04 & 0.09 & -0.12 & 0.13 & 0.24 & 0.40 & 0.06 & 0.01 & 0.20 & 0.10 \\
\hline 6 & 0.34 & 0.74 & 0.03 & 0.01 & -0.72 & 0.66 & 0.06 & 0.11 & 0.03 & 0.06 & -0.14 & -0.01 & 0.04 & 0.10 & -0.13 & 0.14 & 0.26 & 0.42 & 0.06 & 0.02 & 0.20 & 0.11 \\
\hline 7 & 0.89 & 1.69 & 0.00 & 0.70 & -0.72 & 0.58 & 0.02 & 0.29 & -0.02 & 0.05 & -0.15 & -0.01 & 0.06 & 0.05 & -0.11 & 0.17 & 0.27 & 0.31 & 0.03 & 0.00 & 0.29 & 0.10 \\
\hline 8 & 0.54 & 0.23 & 0.78 & 0.87 & 0.43 & 1.92 & 0.11 & 0.22 & 0.36 & 0.58 & 0.15 & 0.07 & 0.09 & 0.01 & 0.08 & -0.07 & 0.45 & 0.17 & 0.07 & 0.01 & 0.31 & 0.05 \\
\hline 9 & 0.12 & 0.44 & 0.13 & 0.18 & -0.75 & 0.83 & 0.13 & 0.04 & 0.05 & 0.11 & -0.21 & -0.15 & 0.14 & 0.01 & -0.15 & 0.08 & 0.48 & 0.72 & -0.13 & -0.09 & 0.18 & 0.09 \\
\hline 10 & 0.17 & 0.63 & 0.17 & 0.11 & -0.47 & 0.69 & 0.09 & 0.04 & -0.08 & 0.17 & -0.12 & -0.04 & 0.05 & 0.04 & -0.08 & 0.09 & 0.21 & 0.28 & -0.08 & 0.00 & 0.18 & 0.09 \\
\hline 11 & 0.88 & 1.53 & 0.15 & 10.50 & -0.80 & 0.58 & 0.07 & 0.21 & 0.25 & 0.40 & -0.09 & -0.01 & 0.04 & 0.03 & -0.06 & 0.09 & 0.35 & 0.16 & -0.05 & 0.03 & 0.26 & 0.06 \\
\hline 12 & 0.32 & 0.33 & 0.47 & 0.28 & -0.20 & 0.22 & 0.08 & 0.17 & 0.02 & 0.12 & 0.09 & -0.04 & 0.05 & 0.00 & 0.05 & -0.12 & 0.21 & 0.01 & -0.06 & -0.00 & 0.19 & 0.03 \\
\hline 13 & 0.48 & 0.11 & 0.33 & 0.56 & 0.30 & 0.20 & 0.13 & 0.30 & 0.18 & 0.37 & 0.00 & -0.07 & 0.00 & -0.04 & 0.04 & -0.08 & 0.04 & 0.02 & -0.09 & -0.01 & - & 0.00 \\
\hline 14 & & 0.23 & & 4.32 & 0.72 & 0.46 & 0.11 & 0.02 & 0.35 & 0.82 & 0.00 & 0.03 & 0.01 & 0.02 & -0.01 & -0.03 & 0.15 & 0.11 & 0.09 & 0.03 & 0.03 & 0.01 \\
\hline 15 & 0.01 & 0.22 & 3.03 & 0.10 & 0.44 & 0.23 & 0.02 & 0.09 & -0.02 & 0.04 & 0.01 & 0.01 & 0.00 & -0.01 & 0.01 & -0.02 & 0.03 & 0.07 & 0.02 & -0.02 & 0.04 & 0.01 \\
\hline 16 & 0.30 & 0.14 & 0.54 & 0.67 & 1.79 & 0.31 & 0.03 & 0.13 & -0.17 & 0.34 & 0.05 & 0.01 & 0.03 & 0.02 & 0.02 & -0.04 & 0.07 & -0.01 & 0.03 & -0.00 & 0.06 & -0.02 \\
\hline
\end{tabular}

Table 46: Frequency domain parameters SESSION 4 - SESSION 1 difference for GAv ERP - EAE Therapy

Time domain parameters

\begin{tabular}{|c|c|c|c|c|c|c|c|c|c|c|c|c|c|c|c|c|c|c|c|c|c|c|c|c|}
\hline channel & Mean & MeanAbs & Median & P1_ampl & \begin{tabular}{|l} 
P1_latency \\
\end{tabular} & N1_ampl & N1_latency & P2/3_ampl & P2/3_latency & SSI & IEEG & $\mathrm{WL}$ & \begin{tabular}{|l|} 
MMAV1 \\
\end{tabular} & MMAV2 & STD DEV & VAR & ROOT & RMS & CREST & SKEWNESS & KURTOSIS & CLEARANCE & SHAPE & IMPULSE \\
\hline 1 & 2.98 & 0.01 & 0.23 & 0.33 & 0.11 & \begin{tabular}{|l|l|}
0.00 \\
\end{tabular} & & 0.08 & 0.11 & 0.05 & 0.01 & -0.20 & $\begin{array}{l}-0.02 \\
\end{array}$ & -0.06 & \begin{tabular}{|l|}
0.02 \\
\end{tabular} & 0.04 & 0.03 & 0.02 & -0.02 & 0.02 & 0.01 & 0.04 & \begin{tabular}{|l|l|} 
& 0.03 \\
\end{tabular} & 0.01 \\
\hline 2 & 2.92 & 0.00 & 0.72 & 0.58 & 0.04 & 0.31 & 0.05 & 0.06 & 0.12 & 0.15 & 0.00 & -0.20 & 0.00 & 0.05 & 0.07 & 0.15 & 0.05 & 0.07 & 0.22 & 0.14 & 0.18 & 0.38 & 0.07 & 0.30 \\
\hline 3 & 0.72 & 0.6 & 1.34 & 0.50 & 0.14 & 0.00 & 0.05 & 0.14 & 0.14 & 0.18 & 0.08 & -0.11 & 0.08 & 0.05 & 0.08 & 0.17 & 0.09 & 0.09 & -0.08 & 0.10 & 0.02 & 0.08 & 0.01 & 0.07 \\
\hline 4 & 0.70 & 0.00 & 0.53 & 0.21 & 0.11 & 0.03 & - & 0.01 & 0. & 0.08 & 0.00 & -0.21 & 0.00 & 0.03 & 0.04 & 0.08 & 0.06 & 0.04 & -0.00 & 0.09 & 0.01 & 0.11 & 0.04 & 0.04 \\
\hline 5 & 0.92 & 0.6 & 0.50 & 0.23 & & 0.05 & - & 0.07 & 0. & 0.09 & 0.01 & -0.14 & 0.00 & 0.02 & 0.04 & 0.08 & 0.07 & 0.04 & 0.0 & 0.14 & 0.03 & 0.13 & 0.05 & 0.06 \\
\hline 6 & & & & 0.30 & & 0 & & & & 0.09 & 0.01 & -0.13 & & 0.05 & 0.0 & 0.09 & 0.06 & 0.05 & 0. & & & 0.14 & 0.05 & 0.08 \\
\hline 7 & 10.00 & 0.07 & 0.65 & 0.47 & 0.15 & 0.22 & 0.07 & 0.04 & 0.01 & 0.24 & 0.07 & -0.15 & 0.06 & 0.02 & 0.11 & 0.24 & 0.05 & 0.12 & 0.09 & 0.18 & 0.21 & 0.16 & 0.04 & 0.14 \\
\hline 8 & 0.04 & & & 0.23 & & 0.08 & 0.46 & & & 0.08 & 0.04 & 0.32 & 0.07 & 0.11 & 0.04 & 0.08 & 0.03 & 0.04 & 0.0 & 0.82 & 0.08 & 0.03 & -0.00 & 0.04 \\
\hline 9 & 1.65 & 0.09 & & 0.02 & 0 & 0.01 & 0.03 & 0.0 & 0. & 0.01 & 0.09 & -0.26 & 0.08 & 0.13 & 0.00 & 0.01 & 0.17 & 0.00 & -0.0 & 0.13 & 0.11 & 0.19 & 0.10 & 0.08 \\
\hline 10 & 6.66 & & 0. & 0.20 & & 0.14 & & 0.14 & & 0.02 & 0.09 & -0.12 & & 0.13 & 0.00 & 0.00 & 0.15 & 0.01 & 0.1 & 0.2 & & 0.35 & 0.11 & 0.25 \\
\hline 11 & 2.1 & & & 0.3 & & 0.34 & 0.14 & & & 0.1 & 0.03 & -0.18 & & 0.03 & 0.05 & 0.10 & 0.03 & 0.05 & 0.1 & & .14 & 0.16 & 0.02 & 0.17 \\
\hline 12 & 0.77 & 0.6 & & 0.02 & 0.5 & 0.19 & 0.21 & & 0.2 & 0.17 & 0.09 & 0.05 & 0.08 & 0.06 & 0.08 & 0.15 & 0.08 & 0.09 & 0.1 & 0.3 & & 0.11 & 0.00 & 0.12 \\
\hline 13 & 2.11 & 0. & & 0.2 & 0. & 0.07 & 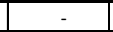 & 0.0 & 0.0 & 0.29 & 0.22 & -0.01 & 0. & 0.16 & 0.16 & 0.29 & 0.25 & 0.16 & 0.27 & 0.17 & .31 & 0.42 & 0.08 & 0.37 \\
\hline 14 & 1.13 & 0.07 & $\begin{array}{l}1.1 \\
\end{array}$ & 0.07 & 0.07 & 0.27 & & 0.45 & 0.26 & 0.17 & 0.07 & -0.18 & 0.09 & 0.09 & 0.00 & 0.00 & 0.06 & 0.09 & -0.09 & 1.43 & 0.00 & 0.12 & -0.02 & 0.11 \\
\hline 15 & 1.2 & 0.0 & 1.2 & 0.2 & & 1.94 & & 0.6 & 0.0 & 0.0 & 0.01 & 0.1 & 0.4 & 0.07 & 0.05 & 0.0 & 0.02 & 0.02 & 0.31 & 4.98 & 0.33 & 0.35 & 0.03 & 0.34 \\
\hline 16 & 1.20 & 0.07 & 1.29 & 0.43 & 0.07 & 2.92 & 0.03 & 0.12 & 0.32 & 0.10 & 0.07 & 0.05 & 0.08 & 0.05 & 0.00 & 0.00 & 0.06 & 0.05 & 0.09 & 3.04 & 0.22 & 0.10 & 0.02 & 0.11 \\
\hline
\end{tabular}

Table 47: Time domain parameters SESSION 4 - SESSION 1 difference for GAv ERP - EAE Therapy 